CS $-89-85$

\title{
Performance of Various Computers Using Standard Linear Equations Software
}

\author{
Jack J. Dongarra* \\ Electrical Engineering and Computer Science Department \\ University of Tennessee \\ Knoxville, TN 37996-1301 \\ Computer Science and Mathematics Division \\ Oak Ridge National Laboratory \\ Oak Ridge, TN 37831 \\ University of Manchester
}

CS $-89-85$

June 15, 2014

* Electronic mail address: dongarra@eecs.utk.edu.

An up-to-date version of this report can be found at http://www.netlib.org/benchmark/performance.ps This work was supported in part by the Applied Mathematical Sciences subprogram of the Office of Energy Research, U.S. Department of Energy, under Contract DE-AC05-96OR22464, and in part by the Science Alliance a state supported program at the University of Tennessee. 


\title{
Performance of Various Computers Using Standard Linear Equations Software
}

\author{
Jack J. Dongarra \\ Electrical Engineering and Computer Science Department \\ University of Tennessee \\ Knoxville, TN 37996-1301 \\ Computer Science and Mathematics Division \\ Oak Ridge National Laboratory \\ Oak Ridge, TN 37831 \\ University of Manchester
}

June 15, 2014

\begin{abstract}
This report compares the performance of different computer systems in solving dense systems of linear equations. The comparison involves approximately a hundred computers, ranging from the Earth Simulator to personal computers.
\end{abstract}

\section{Introduction and Objectives}

The timing information presented here should in no way be used to judge the overall performance of a computer system. The results reflect only one problem area: solving dense systems of equations.

This report provides performance information on a wide assortment of computers ranging from the home-used PC up to the most powerful supercomputers. The information has been collected over a period of time and will undergo change as new machines are added and as hardware and software systems improve. The programs used to generate this data can easily be obtained over the Internet. While we make every attempt to verify the results obtained from users and vendors, errors are bound to exist and should be brought to our attention. We encourage users to obtain the programs and run the routines on their machines, reporting any discrepancies with the numbers listed here.

The first table reports three numbers for each machine listed (in some cases the numbers are missing because of lack of data). All performance numbers reflect an accuracy of full precision (usually 64-bit), unless noted. On some machines full precision may be single precision, such as the Cray, or double precision, such as the IBM. The first number is for the LINPACK [1] benchmark program for a matrix of order 100 in a Fortran environment. The second number is for solving a system of equations of order 1000, with no restriction on the method or its implementation. The third number is the theoretical peak performance of the machine.

LINPACK programs can be characterized as having a high percentage of floating-point arithmetic operations. The routines involved in this timing study, SGEFA and SGESL, use column-oriented algorithms. That is, the programs usually reference array elements sequentially down a column, not across a row. Column orientation is important in increasing efficiency because of the way Fortran stores arrays. Most floating-point operations in LINPACK take place in a set of subprograms, the Basic Linear Algebra Subprograms (BLAS) [3], which are called 
repeatedly throughout the calculation. These BLAS, referred to now as Level 1 BLAS, reference one-dimensional arrays, rather than two-dimensional arrays.

In the first case, the problem size is relatively small (order 100), and no changes were made to the LINPACK software. Moreover, no attempt was made to use special hardware features or to exploit vector capabilities or multiple processors. (The compilers on some machines may, of course, generate optimized code that itself accesses special features.) Thus, many high-performance machines may not have reached their asymptotic execution rates.

In the second case, the problem size is larger (matrix of order 1000), and modifying or replacing the algorithm and software was permitted to achieve as high an execution rate as possible. Thus, the hardware had more opportunity for reaching near-asymptotic rates. An important constraint, however, was that all optimized programs maintain the same relative accuracy as standard techniques, such as Gaussian elimination used in LINPACK.

Furthermore, the driver program (supplied with the LINPACK benchmark) had to be run to ensure that the same problem is solved. The driver program sets up the matrix, calls the routines to solve the problem, verifies that the answers are correct, and computes the total number of operations to solve the problem (independent of the method) as $2 n^{3} / 3+2 n^{2}$, where $n=1000$.

The last column is based not on an actual program run, but on a paper computation to determine the theoretical peak Mflop/s rate for the machine. This is the number manufacturers often cite; it represents an upper bound on performance. That is, the manufacturer guarantees that programs will not exceed this rate- sort of a "speed of light" for a given computer.

The theoretical peak performance is determined by counting the number of floating-point additions and multiplications (in full precision) that can be completed during a period of time, usually the cycle time of the machine. As an example, the Cray Y-MP/8 has a cycle time of 6 ns. During a cycle the results of both an addition and a multiplication can be completed $\frac{2 \text { operations }}{1 \text { cycle }} * \frac{1 \text { cycle }}{6 \mathrm{~ns}}=333 \mathrm{Mflop} / \mathrm{s}$ on a single processor. On the Cray $\mathrm{Y}-\mathrm{MP} / 8$ there are 8 processors; thus, the peak performance is $2667 \mathrm{Mflop} / \mathrm{s}$.

The information in this report is presented to users to provide a range of performance for the various computers and to show the effects of typical Fortran programming and the results that can be obtained through careful programming. The maximum rate of execution is given for comparison. The column labeled "Computer" gives the name of the computer hardware on which the program was run. In some cases we have indicated the number of processors in the configuration and, in some cases, the cycle time of the processor in nanoseconds.

The column labeled "LINPACK Benchmark" gives the operating system and compiler used. The run was based on two routines from LINPACK: SGEFA and SGESL were used for single precision, and DGEFA and DGESL were used for double precision. These routines perform standard LU decomposition with partial pivoting and backsubstitution. The timing was done on a matrix of order 100, where no changes are allowed to the Fortran programs.

The column labeled "TPP" (Toward Peak Performance) gives the results of hand optimization; the problem size was of order 1000.

The final column labeled "Theoretical Peak" gives the maximum rate of execution based on the cycle time of the hardware.

The same matrix was used to solve the system of equations. The results were checked 
for accuracy by calculating a residual for the problem $\|A x-b\| /(\|A\|\|\mid x\|)$. The residual must be less than $n \varepsilon$ where $n$ is the order of the matrix and $\varepsilon$ is the machine precision, on IEEE computers this is $2^{-53}$.

The term Mflop/s, used as a rate of execution, stands for millions of floating-point operations completed per second. For solving a system of $n$ equations, $2 / 3 n^{3}+2 n^{2}$ operations are performed (we count both additions and multiplications).

The information in the tables was compiled over a period of time. Subsequent systems software and hardware changes may alter the timings to some extent.

One further note: The following tables should not be taken too seriously. In multiprogramming environments it is often difficult to reliably measure the execution time of a single program. We trust that anyone actually evaluating machines and operating systems will gather more reliable and more representative data.

\section{A Look at Parallel Processing}

While collecting the data presented in Table 1, we were able to experiment with parallel processing on a number of computer systems. For these experiments, we used either the standard LINPACK algorithm or an algorithm based on matrix-matrix [2] techniques. In the case of the LINPACK algorithm, the loop around the SAXPY can be performed in parallel. In the matrix-matrix implementation the matrix product can be split into submatrices and performed in parallel. In either case, the parallelism follows a simple fork-and-join model where each processor gets some number of operations to perform.

For a problem of size 1000, we expect a high degree of parallelism. Thus, it is not surprising that we get such high efficiency (see Table 2). The actual percentage of parallelism, of course, depends on the algorithm and on the speed of the uniprocessor on the parallel part relative to the speed of the uniprocessor on the non-parallel part.

\section{Highly Parallel Computing}

With the arrival of massively parallel computers there is a need to benchmark such machines on problems that make sense. The problem size and rule for the runs reflected in the Tables 1 and 2 do not permit massively parallel computers to demonstrate their potential performance. The basic flaw is the problem size is too small. To provide a forum for comparing such machines the following benchmark was run on a number of massively parallel machines. The benchmark involves solving a system of linear equations (as was done in Tables 1 and 2). However in this case, the problem size is allowed to increase and the performance numbers reflect the largest problem run on the machine.

The ground rules are as follows: Solve systems of linear equations by some method, allow the size of the problem to vary, and measure the execution time for each size problem. In computing the floating-point execution rate, use $2 n^{3} / 3+2 n^{2}$ operations independent of the actual method used. (If you choose to do Gaussian Elimination, partial pivoting must be used.) Compute and report a residual for the accuracy of solution as $\|A x-b\| /(\|A\|\|x\|)$. The residual must be less than $n \varepsilon$ where $n$ is the order of the matrix and $\varepsilon$ is the machine precision, on IEEE computers this is $2^{-53}$.

The columns in Table 3 are defined as follows:

$R_{\max }$ the performance in Gflop/s for the largest problem run on a machine. 
$N_{\max }$ the size of the largest problem run on a machine.

$N_{1 / 2}$ the size where half the $R_{\max }$ execution rate is achieved.

$R_{\text {peak }}$ the theoretical peak performance in Gflop/s for the machine.

In addition, the number of processors and the cycle time is listed.

\section{Obtaining the Software and Running the Benchmarks}

The software used to generate the data for this report can be obtained by sending electronic mail to netlib@ornl.gov.

\section{LINPACK Benchmark}

The first results listed in Table 1 involved no hand optimization of the LINPACK benchmark.

To receive the single-precision software for this benchmark, in the mail message to netlib@ornl.gov type:

send linpacks from benchmark .

To receive the double-precision software for the LINPACK Benchmark, type:

send linpackd from benchmark .

To run the timing programs, one must supply a real function SECOND which returns the time in seconds from some fixed starting time.

There is only one ground rule for running this benchmark:

- No changes are to be made to the Fortran source code, not even changes in the comments.

The compiler and operating system must be generally available. Results from a beta version of a compiler are allowed, however the standard compiler results must also be listed.

\section{Toward Peak Performance}

The second set of results listed in Table 1 reflected user optimization of the software.

To receive the single-precision software for the column labeled "Toward Peak Performance," in the mail message netlib@ornl.gov type:

send 1000s from benchmark

To receive the double-precision software, type:

send 1000d from benchmark

The ground rules for running this benchmark are as follows:

- Replacements or modifications are allowed in the routine LU.

- The user is allowed to supply any method for the solution of the system of equations.

- The Mflop/s rate will be computed based on the operation count for LU decomposition.

- In all cases, the main driver routine, with its test matrix generator and residual check, must be used.

This report is updated from time to time. A fax copy of this report can be supplied, for details contact the author. To obtain a Postscript copy of the report send mail to netlib@ornl.gov and in the message type:

send performance from benchmark.

To have results verified, please send the output of the runs to

Jack Dongarra 
Computer Science Department

University of Tennessee

Knoxville, TN 37996-1301

Email: dongarra@cs.utk.edu

There is a "Frequently Asked Questions" file for the Linpack benchmark and Top500 at http://www.netlib.org/utk/people/JackDongarra/faq-linpack.html. 


\section{Table 1: Performance in Solving a System of Linear Equations}

\begin{tabular}{|c|c|c|c|c|}
\hline Computer & $\begin{array}{c}\text { "LINPACK Benchmark" } \\
\text { OS/Compiler }\end{array}$ & $\begin{array}{c}n=100 \\
\text { Mflop/s }\end{array}$ & $\begin{array}{c}\text { "TPP" } \\
\text { Best } \\
\text { Effort } \\
\text { n=1000 } \\
\text { Mflop/s }\end{array}$ & $\begin{array}{l}\text { "Theoritical } \\
\text { Peak" } \\
\text { Mflop/s }\end{array}$ \\
\hline Intel Pentium Woodcrest (1 core, $3 \mathrm{GHz}$ ) & $\begin{array}{l}\text { ifort -parallel -xT -O3 -ipo } \\
\text {-mP2OPT_hlo_loop_unroll_factor=2 }\end{array}$ & 3018 & 6542 & 12000 \\
\hline Intel Pentium Woodcrest (1 core, $2.67 \mathrm{GHz})$ & ifort -O3 -ipo -xT -r8 -i8 & 2636 & & 10680 \\
\hline Intel Core 2 Q6600 Kensfield) (4 core, $2.4 \mathrm{GHz}$ ) & & & 13130 & 38400 \\
\hline Intel Core 2 Q6600 Kensfield) (3 core, $2.4 \mathrm{GHz}$ ) & & & 11980 & 28800 \\
\hline Intel Core 2 Q6600 Kensfield) (2 core, $2.4 \mathrm{GHz}$ ) & & & 9669 & 19200 \\
\hline Intel Core 2 Q6600 Kensfield) (1 core, $2.4 \mathrm{GHz}$ ) & $\begin{array}{l}\text { ifort }-\mathrm{O} 3 \text {-xT -ipo -static }-\mathrm{i} 8 \\
\text {-mP2OPT_hlo_loop_unroll_factor }=2\end{array}$ & 2426 & 7519 & 9600 \\
\hline NEC SX-8/8 (8proc. $2 \mathrm{GHz})$ & & & 75140 & 128000 \\
\hline NEC SX-8/4 (4proc. $2 \mathrm{GHz})$ & & & 43690 & 64000 \\
\hline NEC SX-8/2 (2proc. $2 \mathrm{GHz})$ & & & 25060 & 32000 \\
\hline NEC SX-8/1 (1proc. $2 \mathrm{GHz})$ & -pi -Wf"-prob_use" & 2177 & 14960 & 16000 \\
\hline $\begin{array}{l}\text { HCL Infiniti Global Line } 4700 \text { HW ( } 4 \text { proc Intel } \\
\text { Xeon } 3.16 \mathrm{GHz} \text { ) }\end{array}$ & ifort -fast -r8 -align & 1892 & 9917 & 25280 \\
\hline $\begin{array}{l}\text { HP ProLiant BL20p G3 (2 proc (1 cpu core per } \\
\text { single chip), 3.8GHz Intel Xeon) }\end{array}$ & & & 8185 & 14800 \\
\hline $\begin{array}{l}\text { HP ProLiant BL20p G3 (1 proc (1 cpu core per } \\
\text { single chip), 3.8GHz Intel Xeon) }\end{array}$ & $\begin{array}{l}\text { SuSE SLES } 9 \text { / Intel } 8.1 \text { Compile flags: } \\
\text {-fpp -xP -O3 -openmp -align -ipo }\end{array}$ & 1852 & 4851 & 7400 \\
\hline $\begin{array}{l}\text { HP ProLiant DL } 360 \text { G4 }(2 \text { proc, } 3.6 \mathrm{GHz} / 1 \mathrm{MB} \\
\text { Xeon) }\end{array}$ & & & 7031 & 14400 \\
\hline $\begin{array}{l}\text { HP ProLiant DL360 G4 (1 proc, } 3.6 \mathrm{GHz} / 1 \mathrm{MB} \\
\text { Xeon) }\end{array}$ & $\begin{array}{l}\text { Intel } 8.1 \text {-fpp -xW -O2 -unroll -align } \\
\text {-openmp }\end{array}$ & 1821 & 4220 & 7200 \\
\hline $\begin{array}{l}\text { HP ProLiant DL360 G4p ( } 2 \text { proc ( } 1 \text { cpu core per } \\
\text { single chip), } 3.8 \mathrm{GHz} \text { Intel Xeon) }\end{array}$ & & & 8155 & 14800 \\
\hline $\begin{array}{l}\text { HP ProLiant DL360 G4p (1 proc ( } 1 \text { cpu core per } \\
\text { single chip), } 3.8 \mathrm{GHz} \text { Intel Xeon) }\end{array}$ & $\begin{array}{l}\text { SuSE SLES } 9 \text { / Intel } 8.1 \text { Compile flags: } \\
\text {-fpp -xP -O3 -openmp -align -ipo }\end{array}$ & 1861 & 4860 & 7400 \\
\hline $\begin{array}{l}\text { HP ProLiant DL140 G2 (2 proc (1 cpu core per } \\
\text { single chip), } 3.8 \mathrm{GHz} \text { Intel Xeon) }\end{array}$ & & & 8163 & 14800 \\
\hline $\begin{array}{l}\text { HP ProLiant DL140 G2 (1 proc (1 cpu core per } \\
\text { single chip), } 3.8 \mathrm{GHz} \text { Intel Xeon) }\end{array}$ & $\begin{array}{l}\text { SuSE SLES } 9 \text { / Intel } 8.1 \text { Compile flags: } \\
\text {-fpp -xP -O3 -openmp -align -ipo }\end{array}$ & 1861 & 4858 & 7400 \\
\hline $\begin{array}{l}\text { HP ProLiant ML370 G4 ( } 2 \text { proc ( } 1 \text { cpu core per } \\
\text { single chip), } 3.8 \mathrm{GHz} \text { Intel Xeon) }\end{array}$ & & & 8111 & 14800 \\
\hline $\begin{array}{l}\text { HP ProLiant ML370 G4 (1 proc ( } 1 \text { cpu core per } \\
\text { single chip), } 3.8 \mathrm{GHz} \text { Intel Xeon) }\end{array}$ & $\begin{array}{l}\text { SuSE SLES } 9 \text { / Intel } 8.1 \text { Compile flags: } \\
\text {-fpp -xP -O3 -openmp -align -ipo }\end{array}$ & 1851 & 4835 & 7400 \\
\hline $\begin{array}{l}\text { HP ProLiant DL380 G4 ( } 2 \text { proc ( } 1 \text { cpu core per } \\
\text { single chip), } 3.8 \mathrm{GHz} \text { Intel Xeon) }\end{array}$ & & & 8198 & 14800 \\
\hline $\begin{array}{l}\text { HP ProLiant DL } 380 \mathrm{G} 4 \text { ( } 1 \text { proc ( } 1 \mathrm{cpu} \text { core per } \\
\text { single chip), } 3.8 \mathrm{GHz} \text { Intel Xeon) }\end{array}$ & $\begin{array}{l}\text { SuSE SLES } 9 \text { / Intel } 8.1 \text { Compile flags: } \\
\text {-fpp -xP -O3 -openmp -align -ipo }\end{array}$ & 1851 & 4882 & 7400 \\
\hline Intel Pentium Nocona $3.6 \mathrm{GHz}$ & ifort -O3 - xP -ipo -align -r8 & 1803 & 3385 & 7200 \\
\hline Intel xeon 64 (dual) $3.6 \mathrm{GHz}$ & ifort -fast -r8 -align. & 1779 & 7278 & 14400 \\
\hline IBM eServer p5 575 (8 proc, 1.9 GHz POWER5) & & & 34570 & 60800 \\
\hline IBM eServer p5 575 (1 proc, 1.9 GHz POWER5) & $\begin{array}{l}-\mathrm{O} 3 \text {-qarch=pwr5 -qtune=pwr5 }-\mathrm{Pv} \\
-\mathrm{Wp},- \text { ea478,-g1 }\end{array}$ & 1776 & 5872 & 7600 \\
\hline
\end{tabular}




\begin{tabular}{|c|c|c|c|c|}
\hline Computer & $\begin{array}{c}\text { "LINPACK Benchmark" } \\
\text { OS/Compiler }\end{array}$ & $\begin{array}{c}n=100 \\
\text { Mflop/s }\end{array}$ & $\begin{array}{c}\text { "TPP" } \\
\text { Best } \\
\text { Effort } \\
\text { n=1000 } \\
\text { Mflop/s }\end{array}$ & \begin{tabular}{|c} 
"Theoritical \\
Peak" \\
Mflop/s
\end{tabular} \\
\hline SGI Altix $3700 \mathrm{Bx} 2$ Itanium $2(1$ proc $1.6 \mathrm{GHz})$ & $\begin{array}{l}\text {-ipo -O3 -mP2OPT_hlo_loadpair=F } \\
\text {-mP2OPT_hlo_prefetch=F } \\
\text {-mP2OPT_hlo_loop_unroll_factor }=2 \\
\text {-mP3OPT_ecg_mm_fp_ld_latency }=8\end{array}$ & 1765 & 5953 & 6400 \\
\hline $\begin{array}{l}\text { HP Integrity rx2620-2 (2 proc, } 1.6 \mathrm{GHz} / 3 \mathrm{MB} \\
\text { Itanium 2) }\end{array}$ & & & 10210 & 12800 \\
\hline $\begin{array}{l}\text { HP Integrity rx2620-2 (1 proc, } 1.6 \mathrm{GHz} / 3 \mathrm{MB} \\
\text { Itanium 2) }\end{array}$ & $\begin{array}{l}\text { HP-UX, f90 +Ofaster +Oloop_unroll }=2 \\
+ \text { +Onodataprefetch }\end{array}$ & 1761 & 5603 & 6400 \\
\hline $\begin{array}{l}\text { HP Integrity rx1620-2 (2 proc, } 1.6 \mathrm{GHz} / 3 \mathrm{MB} \\
\text { Itanium 2) }\end{array}$ & & & 10320 & 12800 \\
\hline $\begin{array}{l}\text { HP Integrity rx1620-2 (1 proc, } 1.6 \mathrm{GHz} / 3 \mathrm{MB} \\
\text { Itanium 2) }\end{array}$ & $\begin{array}{l}\text { HP-UX, f90 +Ofaster +Oloop_unroll }=2 \\
+ \text { +Onodataprefetch }\end{array}$ & 1761 & 5655 & 6400 \\
\hline $\begin{array}{l}\text { HP ProLiant DL140 G2 (2 proc (1 cpu core per } \\
\text { single chip), 3.6GHz Intel Xeon) }\end{array}$ & & & 7870 & 14400 \\
\hline $\begin{array}{l}\text { HP ProLiant DL140 G2 (1 proc (1 cpu core per } \\
\text { single chip), 3.6GHz Intel Xeon) }\end{array}$ & $\begin{array}{l}\text { SuSE SLES } 9 \text { / Intel } 8.1 \text { Compile flags: } \\
\text {-fpp -xP -O3 -openmp -align -ipo }\end{array}$ & 1756 & 4620 & 7200 \\
\hline $\begin{array}{l}\text { HP Integrity rx4640-8 (4 proc, } 1.6 \mathrm{GHz} / 9 \mathrm{MB} \\
\text { Itanium 2) }\end{array}$ & & & 19470 & 25600 \\
\hline $\begin{array}{l}\text { HP Integrity rx4640-8 ( } 2 \text { proc, } 1.6 \mathrm{GHz} / 9 \mathrm{MB} \\
\text { Itanium 2) }\end{array}$ & & & 10940 & 12800 \\
\hline $\begin{array}{l}\text { HP Integrity rx } 4640-8 \text { ( } 1 \text { proc, } 1.6 \mathrm{GHz} / 9 \mathrm{MB} \\
\text { Itanium 2) }\end{array}$ & $\begin{array}{l}\text { HP-UX, f90 +Ofaster +Oloop_unroll }=2 \\
+ \text { +Onodataprefetch }\end{array}$ & 1756 & 5959 & 6400 \\
\hline $\begin{array}{l}\text { HP ProLiant ML350 G4p ( } 2 \text { proc ( } 1 \text { cpu core per } \\
\text { single chip), 3.6GHz Intel Xeon) }\end{array}$ & & & 7876 & 14400 \\
\hline $\begin{array}{l}\text { HP ProLiant ML350 G4p ( } 1 \text { proc ( } 1 \text { cpu core per } \\
\text { single chip), 3.6GHz Intel Xeon) }\end{array}$ & $\begin{array}{l}\text { SuSE SLES } 9 \text { / Intel } 8.1 \text { Compile flags: } \\
\text {-fpp -xP -O3 -openmp -align -ipo }\end{array}$ & 1754 & 4646 & 7200 \\
\hline $\begin{array}{l}\text { HP ProLiant BL20p G3 (2 proc (1 cpu core per } \\
\text { single chip), 3.6GHz Intel Xeon) }\end{array}$ & & & 7851 & 14400 \\
\hline $\begin{array}{l}\text { HP ProLiant BL20p G3 (1 proc (1 cpu core per } \\
\text { single chip), 3.6GHz Intel Xeon) }\end{array}$ & $\begin{array}{l}\text { SuSE SLES } 9 \text { / Intel } 8.1 \text { Compile flags: } \\
\text {-fpp -xP -O3 -openmp -align -ipo }\end{array}$ & 1754 & 4638 & 7200 \\
\hline $\begin{array}{l}\text { HP ProLiant BL45p (4 proc (1 cpu core per } \\
\text { single chip), 2.8GHz AMD } 854 \text { Opteron) }\end{array}$ & & & 12860 & 22400 \\
\hline $\begin{array}{l}\text { HP ProLiant BL45p (2 proc (1 cpu core per } \\
\text { single chip), } 2.8 \mathrm{GHz} \text { AMD } 854 \text { Opteron) }\end{array}$ & & & 7678 & 11200 \\
\hline $\begin{array}{l}\text { HP ProLiant BL45p (1 proc (1 cpu core per } \\
\text { single chip), 2.8GHz AMD } 854 \text { Opteron) }\end{array}$ & $\begin{array}{l}\text { SuSE SLES } 9 \text { / PGI 5.2-4 Compile } \\
\text { Flags: -fastsse -tp k8-64 }\end{array}$ & 1717 & 4191 & 5600 \\
\hline $\begin{array}{l}\text { HP ProLiant BL25p (2 proc (1 cpu core per } \\
\text { single chip), 2.8GHz AMD } 254 \text { Opteron) }\end{array}$ & & & 7683 & 11200 \\
\hline $\begin{array}{l}\text { HP ProLiant BL25p (1 proc (1 cpu core per } \\
\text { single chip), 2.8GHz AMD } 254 \text { Opteron) }\end{array}$ & $\begin{array}{l}\text { SuSE SLES } 9 \text { / PGI 5.2-4 Compile } \\
\text { Flags: -fastsse -tp k8-64 }\end{array}$ & 1717 & 4199 & 5600 \\
\hline $\begin{array}{l}\text { HP ProLiant DL385 (2 proc (1 cpu core per } \\
\text { single chip), 2.8GHz AMD } 254 \text { Opteron) }\end{array}$ & & & 7661 & 11200 \\
\hline $\begin{array}{l}\text { HP ProLiant DL140 G2 (2 proc (1 cpu core per } \\
\text { single chip), } 3.8 \mathrm{GHz} \text { Intel Xeon) }\end{array}$ & & & 8163 & 14800 \\
\hline $\begin{array}{l}\text { HP ProLiant DL585 (4 proc ( } 1 \mathrm{cpu} \text { core per single } \\
\text { chip), } 2.8 \mathrm{GHz} \text { AMD } 854 \text { Opteron) }\end{array}$ & & & 12910 & 22400 \\
\hline $\begin{array}{l}\text { HP ProLiant DL585 ( } 2 \text { proc }(1 \mathrm{cpu} \text { core per single } \\
\text { chip), } 2.8 \mathrm{GHz} \text { AMD } 854 \text { Opteron) }\end{array}$ & & & 7619 & 11200 \\
\hline
\end{tabular}




\begin{tabular}{|c|c|c|c|c|}
\hline Computer & $\begin{array}{c}\text { "LINPACK Benchmark" } \\
\text { OS/Compiler }\end{array}$ & $\begin{array}{c}\mathbf{n}=100 \\
\text { Mflop/s }\end{array}$ & $\begin{array}{c}\text { "TPP" } \\
\text { Best } \\
\text { Effort } \\
\text { n=1000 } \\
\text { Mflop/s }\end{array}$ & $\begin{array}{l}\text { "Theoritical } \\
\text { Peak" } \\
\text { Mflop/s }\end{array}$ \\
\hline $\begin{array}{l}\text { HP ProLiant DL585 ( } 1 \text { proc }(1 \mathrm{cpu} \text { core per single } \\
\text { chip), } 2.8 \mathrm{GHz} \text { AMD } 854 \text { Opteron) }\end{array}$ & $\begin{array}{l}\text { SuSE SLES 9 / PGI 5.2-4 Compile } \\
\text { Flags: -fastsse -tp k8-64 -mp }\end{array}$ & 1712 & 4166 & 5600 \\
\hline $\begin{array}{l}\text { HP ProLiant DL385 (1 proc ( } 1 \mathrm{cpu} \text { core per } \\
\text { single chip), } 2.8 \mathrm{GHz} \text { AMD } 254 \text { Opteron })\end{array}$ & $\begin{array}{l}\text { SuSE SLES 9 / PGI 5.2-4 Compile } \\
\text { Flags: -fastsse -tp k8-64 -mp }\end{array}$ & 1712 & 4238 & 5600 \\
\hline HP ProLiant BL30p (2 proc. $3.20 \mathrm{GHz}$, Xeon) & & & 6264 & 12800 \\
\hline HP ProLiant BL30p (1 proc. $3.20 \mathrm{GHz}$, Xeon) & ifort -xW -O3 -parallel -ipo & 1704 & 3522 & 6400 \\
\hline $\begin{array}{l}\text { IBM eServer BladeCenter JS20 ( } 2 \text { proc, } 2.2 \mathrm{GHz} \\
\text { PowerPC 970) }\end{array}$ & & & 5817 & 17600 \\
\hline $\begin{array}{l}\text { IBM eServer BladeCenter JS20 (1 proc, } 2.2 \mathrm{GHz} \\
\text { PowerPC 970) }\end{array}$ & $-\mathrm{O} 4-$ qarch $=$ auto - qtune $=$ auto & 1681 & 3840 & 8800 \\
\hline $\begin{array}{l}\text { Fujitsu Siemens hpcLine ( } 2 \text { proc Intel Xeon } 3.2 \\
\text { GHz) }\end{array}$ & & & 5151 & 12800 \\
\hline $\begin{array}{l}\text { Fujitsu Siemens hpcLine (1 proc Intel Xeon } 3.2 \\
\text { GHz) }\end{array}$ & ifort -O3 - $\mathrm{xN}$-ipo -align -r8 & 1679 & 3148 & 6400 \\
\hline SGI Altix 3000 (1.5 GHz Itanium 2) & $\begin{array}{l}\text {-O3 -mP2OPT_hlo_loadpair=F } \\
\text {-mP2OPT_hlo_prefetch=F } \\
\text {-mP2OPT_hlo_loop_unroll_factor=2 } \\
\text {-mP3OPT_ecg_mm_fp_ld_latency }=8 \\
\text {-ipo -fno-alias }\end{array}$ & 1659 & 5400 & 6000 \\
\hline HP Integrity Server rx2600 (2 proc $1.5 \mathrm{GHz})$ & & & 10240 & 12000 \\
\hline HP Integrity Server rx2600 (1 proc $1.5 \mathrm{GHz})$ & $\begin{array}{l}\text { f90 +DSitanium } 2+\mathrm{O} 3 \\
\text { +Oinline_budget }=100000 \\
\text { +Ono_ptrs_to_globals +Oloop_unroll=2 } \\
\text { +Onodataprefetch }\end{array}$ & 1635 & 5431 & 6000 \\
\hline HP Integrity Server rx5670 (4 proc $1.5 \mathrm{GHz})$ & & & 18180 & 24000 \\
\hline HP Integrity Server rx5670 (2 proc $1.5 \mathrm{GHz})$ & & & 10030 & 12000 \\
\hline HP Integrity Server rx5670 (1 proc $1.5 \mathrm{GHz})$ & $\begin{array}{l}\text { f90 +DSitanium } 2+\mathrm{O} 3 \\
\text { +Oinline_budget }=100000 \\
\text { +Ono_ptrs_to_globals +Oloop_unroll }=2 \\
\text { +Onodataprefetch }\end{array}$ & 1631 & 5423 & 6000 \\
\hline $\begin{array}{l}\text { HP ProLiant BL } 45 p \text { ( } 4 \text { proc ( } 1 \text { cpu core per single } \\
\text { chip), } 2.6 \mathrm{GHz} 852 \text { Opteron) }\end{array}$ & & & 12030 & 20800 \\
\hline $\begin{array}{l}\text { HP ProLiant BL } 45 p \text { ( } 2 \text { proc ( } 1 \text { cpu core per single } \\
\text { chip), } 2.6 \mathrm{GHz} 852 \text { Opteron) }\end{array}$ & & & 7023 & 10400 \\
\hline $\begin{array}{l}\text { HP ProLiant BL } 45 p \text { ( } 1 \text { proc ( } 1 \text { cpu core per single } \\
\text { chip), } 2.6 \mathrm{GHz} 852 \text { Opteron) }\end{array}$ & PGI 5.2-4 -fastsse -tp k8-64 -mp & 1593 & 3894 & 5200 \\
\hline HP ProLiant BL25p (2 proc, $2.6 \mathrm{GHz}$, Opteron) & & & 7153 & 10400 \\
\hline HP ProLiant BL25p (1 proc, 2.6GHz, Opteron) & PGI 5.2-4 -O2 -tp k8-64 -mp & 1593 & 3938 & 5200 \\
\hline Intel Xeon EM64T (Nocona $3.2 \mathrm{Ghz}$ ) & ifort -fast & 1593 & & 6400 \\
\hline $\begin{array}{l}\text { HP ProLiant DL585 ( } 4 \text { proc ( } 1 \text { cpu core per single } \\
\text { chip), } 2.6 \mathrm{GHz} 852 \text { Opteron) }\end{array}$ & & & 11970 & 20800 \\
\hline $\begin{array}{l}\text { HP ProLiant DL585 ( } 2 \text { proc ( } 1 \text { cpu core per single } \\
\text { chip), 2.6GHz } 852 \text { Opteron) }\end{array}$ & & & 7098 & 10400 \\
\hline $\begin{array}{l}\text { HP ProLiant DL585 ( } 1 \text { proc ( } 1 \text { cpu core per single } \\
\text { chip), } 2.6 \mathrm{GHz} 852 \text { Opteron) }\end{array}$ & PGI 5.2-4 -fastsse -tp k8-64 -mp & 1586 & 3879 & 5200 \\
\hline HP ProLiant DL385 (2 proc, $2.6 \mathrm{GHz}$, Opteron) & & & 7134 & 10400 \\
\hline HP ProLiant DL385 (1 proc, $2.6 \mathrm{GHz}$, Opteron) & PGI 5.2-4 -O2 -tp k8-64 -mp & 1586 & 3917 & 5200 \\
\hline
\end{tabular}




\begin{tabular}{|c|c|c|c|c|}
\hline Computer & $\begin{array}{c}\text { "LINPACK Benchmark" } \\
\text { OS/Compiler }\end{array}$ & $\begin{array}{c}\mathbf{n}=\mathbf{1 0 0} \\
\mathrm{Mflop} / \mathrm{s}\end{array}$ & $\begin{array}{c}\text { “TPP" } \\
\text { Best } \\
\text { Effort } \\
\text { n=1000 } \\
\text { Mflop/s }\end{array}$ & $\begin{array}{l}\text { "Theoritical } \\
\text { Peak" } \\
\text { Mflop/s }\end{array}$ \\
\hline HP ProLiant DL585 (4 proc, 2.6GHz, Opteron) & & & 11450 & 20800 \\
\hline HP ProLiant DL585 (2 proc, 2.6GHz, Opteron) & & & 6913 & 10400 \\
\hline HP ProLiant DL585 (1 proc, 2.6GHz, Opteron) & PGI 5.2-4 -O2 -tp k8-64 -mp & 1586 & 3836 & 5200 \\
\hline Pentium IV with $3.0 \mathrm{GHz}$ & ifort -O3 -xW -ip -ipo -align -pad & 1573 & 3181 & 6000 \\
\hline Intel Pentium 4 3.0 GHz (Northwood core) & ifort -xW -O3 -ipo -static -r8 & 1571 & 3650 & 6000 \\
\hline HP ProLiant BL20p G3 (2 proc 3.6GHZ) & & & 7200 & 14400 \\
\hline HP ProLiant BL20p G3 (1 proc 3.6GHZ) & ifort -fpp -xP -O3 & 1565 & 4403 & 7200 \\
\hline IBM eServer pSeries 655 ( 8 proc $1.7 \mathrm{GHz})$ & & & 25630 & 54400 \\
\hline IBM eServer pSeries 655 (4 proc $1.7 \mathrm{GHz})$ & & & 14730 & 27200 \\
\hline IBM eServer pSeries 655 (1 proc $1.7 \mathrm{GHz})$ & $\begin{array}{l}\text {-O3 -qarch=pwr4 -qtune=pwr4 -Pv } \\
-\mathrm{Wp},- \text { ea478,-g1 }\end{array}$ & 1486 & 3884 & 6800 \\
\hline $\begin{array}{l}\text { HP ProLiant BL45p ( } 8 \text { proc }(2 \text { cpu cores per } \\
\text { single chip), 2.4GHz AMD } 880 \text { Opteron })\end{array}$ & & & 15830 & 38400 \\
\hline $\begin{array}{l}\text { HP ProLiant BL45p ( } 4 \text { proc ( } 2 \text { cpu cores per } \\
\text { single chip), 2.4GHz AMD } 880 \text { Opteron) }\end{array}$ & & & 11460 & 19200 \\
\hline $\begin{array}{l}\text { HP ProLiant BL45p ( } 2 \text { proc }(2 \text { cpu cores per } \\
\text { single chip), 2.4GHz AMD } 880 \text { Opteron })\end{array}$ & & & 6626 & 9600 \\
\hline $\begin{array}{l}\text { HP ProLiant BL45p ( } 1 \text { proc }(2 \text { cpu cores per } \\
\text { single chip), 2.4GHz AMD } 880 \text { Opteron })\end{array}$ & $\begin{array}{l}\text { SuSE SLES } 9 \text { / PGI 5.2-4 Compile } \\
\text { Flags: -fastsse -tp k8-64 }\end{array}$ & 1473 & 3604 & 4800 \\
\hline $\begin{array}{l}\text { HP ProLiant BL25p ( } 4 \text { proc }(2 \text { cpu cores per } \\
\text { single chip), 2.4GHz AMD } 280 \text { Opteron) }\end{array}$ & & & 11590 & 19200 \\
\hline $\begin{array}{l}\text { HP ProLiant BL25p (2 proc ( } 2 \text { cpu cores per } \\
\text { single chip), 2.4GHz AMD } 280 \text { Opteron })\end{array}$ & & & 6715 & 9600 \\
\hline $\begin{array}{l}\text { HP ProLiant BL25p ( } 1 \text { proc }(2 \text { cpu cores per } \\
\text { single chip), 2.4GHz AMD } 280 \text { Opteron })\end{array}$ & $\begin{array}{l}\text { SuSE SLES } 9 \text { / PGI 5.2-4 Compile } \\
\text { Flags: -fastsse -tp k8-64 }\end{array}$ & 1471 & 3654 & 4800 \\
\hline IBM eServer pSeries 690 (16 proc $1.7 \mathrm{GHz})$ & & & 36530 & 108800 \\
\hline IBM eServer pSeries 690 ( 8 proc $1.7 \mathrm{GHz})$ & & & 25130 & 54400 \\
\hline $\begin{array}{l}\text { HP ProLiant DL385 ( } 4 \text { proc }(2 \mathrm{cpu} \text { cores per single } \\
\text { chip), 2.4GHz AMD } 280 \text { Opteron) }\end{array}$ & & & 11570 & 19200 \\
\hline $\begin{array}{l}\text { HP ProLiant DL385 ( } 2 \text { proc ( } 2 \text { cpu cores per single } \\
\text { chip), 2.4GHz AMD } 280 \text { Opteron) }\end{array}$ & & & 6662 & 9600 \\
\hline $\begin{array}{l}\text { HP ProLiant DL385 ( } 1 \text { proc }(2 \mathrm{cpu} \text { cores per single } \\
\text { chip), 2.4GHz AMD } 280 \text { Opteron) }\end{array}$ & $\begin{array}{l}\text { SuSE SLES } 9 \text { / PGI 5.2-4 Compile } \\
\text { Flags: -fastsse -tp k8-64 }\end{array}$ & 1470 & 3657 & 4800 \\
\hline $\begin{array}{l}\text { HP ProLiant DL585 ( } 8 \text { proc ( } 2 \text { cpu cores per single } \\
\text { chip), 2.4GHz AMD } 880 \text { Opteron) }\end{array}$ & & & 15020 & 38400 \\
\hline $\begin{array}{l}\text { HP ProLiant DL585 ( } 4 \text { proc }(2 \mathrm{cpu} \text { cores per single } \\
\text { chip), } 2.4 \mathrm{GHz} \text { AMD } 880 \text { Opteron) }\end{array}$ & & & 11320 & 19200 \\
\hline $\begin{array}{l}\text { HP ProLiant DL585 ( } 2 \text { proc }(2 \mathrm{cpu} \text { cores per single } \\
\text { chip), 2.4GHz AMD } 880 \text { Opteron })\end{array}$ & & & 6566 & 9600 \\
\hline $\begin{array}{l}\text { HP ProLiant DL585 ( } 1 \text { proc ( } 2 \text { cpu cores per single } \\
\text { chip), 2.4GHz AMD } 880 \text { Opteron) }\end{array}$ & $\begin{array}{l}\text { SuSE SLES } 9 \text { / PGI 5.2-4 Compile } \\
\text { Flags: -fastsse -tp k8-64 -mp }\end{array}$ & 1467 & 3581 & 4800 \\
\hline HP DL385 2.2 GHz (dual core) Opteron 275 & ifort -O3 -xW -ipo & 1464 & & 4400 \\
\hline IBM eServer pSeries 690 Tubro ( 1 proc $1.7 \mathrm{GHz}$ ) & $\begin{array}{l}-\mathrm{O} 3 \text {-qarch=pwr4 -qtune=pwr4 -Pv } \\
-\mathrm{Wp},- \text { ea478,-g1 }\end{array}$ & 1462 & 3817 & 6800 \\
\hline HCL Infiniti Global Line 2700HL Xeon EM64T & ifort -fast -ip -ipo -r8 -align & 1444 & 6167 & 9600 \\
\hline
\end{tabular}




\begin{tabular}{|c|c|c|c|c|}
\hline Computer & $\begin{array}{c}\text { "LINPACK Benchmark" } \\
\text { OS/Compiler }\end{array}$ & $\begin{array}{c}n=100 \\
\text { Mflop/s }\end{array}$ & $\begin{array}{c}\text { "TPP" } \\
\text { Best } \\
\text { Effort } \\
\text { n=1000 } \\
\text { Mflop/s }\end{array}$ & $\begin{array}{l}\text { "Theoritical } \\
\text { Peak" } \\
\text { Mflop/s }\end{array}$ \\
\hline \multicolumn{5}{|l|}{ (Dual Core) $2.4 \mathrm{GHz}$} \\
\hline $\begin{array}{l}\text { HCL Infiniti Global Line 2700AF Xeon EM64T } \\
\text { (Dual Core) } 2.4 \mathrm{GHz}\end{array}$ & ifort -fast -ip -ipo -r8 -align & 1438 & 6131 & 9600 \\
\hline $\begin{array}{l}\text { HCL Infiniti Global Line 2700JR2 (Intel Xeon } \\
\text { EM64T } 3.8 \mathrm{GHz} \text { ) }\end{array}$ & ifort -fast -ip -ipo -r8 -align & 1433 & 5144 & 7600 \\
\hline $\begin{array}{l}\text { HCL Infiniti Global Line 2700BD2 (Intel Xeon } \\
\text { 3.16 GHz) }\end{array}$ & ifort -fast -r8 -align -ip -ipo & 1428 & 4474 & 6320 \\
\hline Intel Pentium 4 (3.06 GHz) & ifc -O3 -r8 -xW -ip -ipo -align -pad & 1414 & 2880 & 6120 \\
\hline AMD Opteron $275 / 2.2 \mathrm{Ghz}$ (dual core, 4 proc) & & & 6147 & 17600 \\
\hline AMD Opteron $275 / 2.2 \mathrm{Ghz}$ (dual core, 2 proc) & & & 4630 & 8800 \\
\hline AMD Opteron $275 / 2.2 \mathrm{Ghz}$ (dual core, 1 proc) & ifort -O3 - $\mathrm{xW}$-ipo & 1385 & 2447 & 4400 \\
\hline $\begin{array}{l}\text { HP ProLiant BL } 45 \mathrm{p} \text { ( } 8 \text { proc }(2 \text { cpu cores per single } \\
\text { chip), } 2.2 \mathrm{GHz} 875 \text { Opteron) }\end{array}$ & & & 14120 & 35200 \\
\hline $\begin{array}{l}\text { HP ProLiant BL } 45 \mathrm{p} \text { ( } 4 \text { proc ( } 2 \text { cpu cores per single } \\
\text { chip), } 2.2 \mathrm{GHz} 875 \text { Opteron) }\end{array}$ & & & 10570 & 17600 \\
\hline $\begin{array}{l}\text { HP ProLiant BL } 45 \mathrm{p} \text { ( } 2 \text { proc }(2 \mathrm{cpu} \text { cores per single } \\
\text { chip), } 2.2 \mathrm{GHz} 875 \text { Opteron) }\end{array}$ & & & 6113 & 8800 \\
\hline $\begin{array}{l}\text { HP ProLiant BL } 25 \mathrm{p} \text { ( } 4 \text { proc ( } 2 \text { cpu cores per single } \\
\text { chip), } 2.2 \mathrm{GHz} 275 \text { Opteron) }\end{array}$ & & & 10730 & 17600 \\
\hline $\begin{array}{l}\text { HP ProLiant BL } 25 \mathrm{p} \text { ( } 2 \text { proc ( } 2 \text { cpu cores per single } \\
\text { chip), } 2.2 \mathrm{GHz} 275 \text { Opteron) }\end{array}$ & & & 6158 & 8800 \\
\hline $\begin{array}{l}\text { HP ProLiant BL } 25 \mathrm{p} \text { ( } 1 \text { proc }(2 \mathrm{cpu} \text { cores per single } \\
\text { chip), } 2.2 \mathrm{GHz} 275 \text { Opteron) }\end{array}$ & PGI 5.2-4 -fastsse -tp k8-64 & 1350 & 3347 & 4400 \\
\hline $\begin{array}{l}\text { HP ProLiant DL } 385 \text { ( } 4 \text { proc ( } 2 \text { cpu cores per single } \\
\text { chip), } 2.2 \mathrm{GHz} 275 \text { Opteron) }\end{array}$ & & & 10600 & 17600 \\
\hline $\begin{array}{l}\text { HP ProLiant DL } 385 \text { ( } 2 \text { proc }(2 \text { cpu cores per single } \\
\text { chip), } 2.2 \mathrm{GHz} 275 \text { Opteron) }\end{array}$ & & & 6115 & 8800 \\
\hline $\begin{array}{l}\text { HP ProLiant DL } 385 \text { ( } 1 \text { proc ( } 2 \text { cpu cores per single } \\
\text { chip), } 2.2 \mathrm{GHz} 275 \text { Opteron) }\end{array}$ & PGI 5.2-4 -fastsse -tp k8-64 & 1349 & 3352 & 4400 \\
\hline $\begin{array}{l}\text { HP ProLiant BL } 45 \mathrm{p} \text { ( } 1 \text { proc }(2 \mathrm{cpu} \text { cores per single } \\
\text { chip), } 2.2 \mathrm{GHz} 875 \text { Opteron) }\end{array}$ & PGI 5.2-4 -fastsse -tp k8-64 & 1349 & 3325 & 4400 \\
\hline $\begin{array}{l}\text { HP ProLiant DL } 585 \text { ( } 8 \text { proc ( } 2 \text { cpu cores per single } \\
\text { chip), } 2.2 \mathrm{GHz} 875 \text { Opteron) }\end{array}$ & & & 14040 & 35200 \\
\hline $\begin{array}{l}\text { HP ProLiant DL } 585 \text { ( } 4 \text { proc ( } 2 \text { cpu cores per single } \\
\text { chip), } 2.2 \mathrm{GHz} 875 \text { Opteron) }\end{array}$ & & & 10480 & 17600 \\
\hline $\begin{array}{l}\text { HP ProLiant DL } 585 \text { ( } 2 \text { proc }(2 \text { cpu cores per single } \\
\text { chip), } 2.2 \mathrm{GHz} 875 \text { Opteron) }\end{array}$ & & & 6083 & 8800 \\
\hline $\begin{array}{l}\text { HP ProLiant DL } 585 \text { ( } 1 \text { proc }(2 \text { cpu cores per single } \\
\text { chip), } 2.2 \mathrm{GHz} 875 \text { Opteron) }\end{array}$ & PGI 5.2-4 -fastsse -tp k8-64 & 1348 & 3301 & 4400 \\
\hline Intel Pentium 4 (2.8 GHz) & ifc -O3 -xW -ipo -ip -align & 1317 & 2444 & 5600 \\
\hline IBM eServer p5 575 (1.5 GHz POWER5) & $\begin{array}{l}-\mathrm{O} 3 \quad-\mathrm{qarch}=\text { pwr5 } \\
-\mathrm{Wp},-\mathrm{e} 478,-\mathrm{qtune}=\mathrm{pwr} 1\end{array}$ & 1315 & & 6000 \\
\hline HP ProLiant BL35p (2 proc, $2.4 \mathrm{GHz}$, Opteron) & & & 6460 & 9600 \\
\hline HP ProLiant BL35p (1 proc, $2.4 \mathrm{GHz}$, Opteron) & PGI 5.2-4 -O2 -tp k8-64 -mp & 1300 & 3583 & 4800 \\
\hline IBM eServer pSeries 670 (16 proc $1.5 \mathrm{GHz})$ & & & 33980 & 96000 \\
\hline IBM eServer pSeries 670 ( 8 proc $1.5 \mathrm{GHz})$ & & & 22860 & 48000 \\
\hline IBM eServer pSeries 670 ( 1 proc $1.5 \mathrm{GHz})$ & $-\mathrm{O} 3-\mathrm{qarch}=\mathrm{pwr} 4$-qtune=pwr4 $-\mathrm{Pv}$ & 1294 & 3401 & 6000 \\
\hline
\end{tabular}




\begin{tabular}{|c|c|c|c|c|}
\hline Computer & $\begin{array}{c}\text { "LINPACK Benchmark" } \\
\text { OS/Compiler }\end{array}$ & $\begin{array}{c}\mathbf{n}=100 \\
\text { Mflop/s }\end{array}$ & $\begin{array}{c}\text { "TPP" } \\
\text { Best } \\
\text { Effort } \\
\text { n=1000 } \\
\text { Mflop/s }\end{array}$ & $\begin{array}{l}\text { "Theoritical } \\
\text { Peak" } \\
\text { Mflop/s }\end{array}$ \\
\hline & -Wp,-ea478,-g1 & & & \\
\hline IBM eServer pSeries 655 (8 proc $1.5 \mathrm{GHz})$ & & & 22770 & 48000 \\
\hline IBM eServer pSeries 655 Tubro (1 proc $1.5 \mathrm{GHz}$ ) & $\begin{array}{l}-\mathrm{O} 3 \text {-qarch=pwr4 -qtune=pwr4 -Pv } \\
-\mathrm{Wp},-\mathrm{ea} 478,-\mathrm{g} 1\end{array}$ & 1293 & 3421 & 6000 \\
\hline HP ProLiant DL585 (4 CPU, 2.4GHz 850 Opteron) & $\begin{array}{l}\text { SuSE SLES } 9 \text { / PGI 5.2-4 -O2 -tp k8-64 } \\
\text {-mp }\end{array}$ & & 10540 & 19200 \\
\hline HP ProLiant DL585 (2 CPU, 2.4GHz 850 Opteron) & $\begin{array}{l}\text { SuSE SLES } 9 \text { / PGI 5.2-4 -O2 -tp k8-64 } \\
\text {-mp }\end{array}$ & & 6313 & 9600 \\
\hline HP ProLiant DL585 (1 CPU, 2.4GHz 850 Opteron) & SuSE SLES 9 / PGI 5.2-4 -O2 -tp k8-64 & 1293 & 3489 & 4800 \\
\hline $\begin{array}{l}\text { IBM IntelliStation POWER } 2752 \text { CPUs ( } 1450 \\
\text { MHz POWER4+) }\end{array}$ & & & 5993 & 11600 \\
\hline HP ProLiant DL145 (2 CPU, 2.4GHz 250 Opteron) & $\begin{array}{l}\text { SuSE SLES } 9 \text { / PGI 5.2-4 -O2 -tp k8-64 } \\
\text {-mp }\end{array}$ & & 6369 & 9600 \\
\hline HP ProLiant DL145 (1 CPU, 2.4GHz 250 Opteron) & SuSE SLES 9 / PGI 5.2-4 -O2 -tp k8-64 & 1291 & 3485 & 4800 \\
\hline $\begin{array}{l}\text { IBM IntelliStation POWER } 2751 \text { CPU ( } 1450 \\
\text { MHz POWER4+) }\end{array}$ & $\begin{array}{l}-\mathrm{O} 3-\text {-qarch=pwr4 -qtune=pwr4 -Pv } \\
-\mathrm{Wp},- \text { ea478,-g1 }\end{array}$ & 1245 & 3338 & 5800 \\
\hline IBM eServer pSeries $6306 \mathrm{E} 4$ ( 4 proc $1.45 \mathrm{GHz}$ ) & & & 10990 & 23200 \\
\hline IBM eServer pSeries $6306 \mathrm{E} 4$ (1 proc $1.45 \mathrm{GHz})$ & $\begin{array}{l}\text {-O3 -qarch=pwr4 -qtune=pwr4 -Pv } \\
-\mathrm{Wp},- \text { ea478,-g1 }\end{array}$ & 1229 & 3297 & 5800 \\
\hline IBM eServer pSeries $6306 \mathrm{C} 4$ (4 proc $1.45 \mathrm{GHz})$ & & & 10990 & 23200 \\
\hline IBM eServer pSeries $6306 \mathrm{C} 4$ ( 1 proc $1.45 \mathrm{GHz})$ & $\begin{array}{l}\text {-O3 -qarch=pwr4 -qtune=pwr4 -Pv } \\
-\mathrm{Wp} \text {,-ea478,-g1 }\end{array}$ & 1229 & 3297 & 5800 \\
\hline NEC SX-6/1 ( 8 proc. $1.77 \mathrm{~ns})$ & & & 46260 & 72000 \\
\hline NEC SX-6/1 (4 proc. $1.77 \mathrm{~ns})$ & & & 26540 & 36000 \\
\hline NEC SX-6/1 (2 proc. $1.77 \mathrm{~ns})$ & & & 15020 & 18000 \\
\hline NEC SX-6/1 (1proc. 1.77 ns) & SUPER-UX R13.1 -pi -Wf"-prob_use" & 1289 & 8553 & 9000 \\
\hline AMD Opteron $(2.192 \mathrm{GHz})$ & PGI -fastsse -tp k8-64 & 1253 & 3145 & 4284 \\
\hline IBM eServer pSeries $6506 \mathrm{M} 28 \operatorname{proc}(1450 \mathrm{MHz})$ & & & 19930 & 46400 \\
\hline IBM eServer pSeries $6506 \mathrm{M} 24 \operatorname{proc}(1450 \mathrm{MHz})$ & & & 11190 & 23200 \\
\hline IBM eServer pSeries $6506 \mathrm{M} 22 \operatorname{proc}(1450 \mathrm{MHz})$ & & & 6165 & 11600 \\
\hline IBM eServer pSeries $6506 \mathrm{M} 21 \operatorname{proc}(1450 \mathrm{MHz})$ & $\begin{array}{l}\text {-O3 -qarch=pwr4 -qtune=pwr4 -Pv } \\
-\mathrm{Wp},- \text { ea478,-g1 }\end{array}$ & 1220 & 3245 & 5800 \\
\hline Intel Pentium 4 (2.53 GHz) & ifc -O3 -xW -ipo -ip -align & 1190 & 2355 & 5060 \\
\hline NEC SX-6/8 (8proc. $2.0 \mathrm{~ns})$ & & & 41520 & 64000 \\
\hline NEC SX-6/4 (4proc. $2.0 \mathrm{ns)}$ & & & 23680 & 32000 \\
\hline NEC SX-6/2 (2proc. $2.0 \mathrm{~ns})$ & & & 13350 & 16000 \\
\hline NEC SX-6/1 (1proc. $2.0 \mathrm{~ns})$ & R12.1 -pi -Wf"-prob_use" & 1161 & 7575 & 8000 \\
\hline Fujitsu VPP5000/1(1 proc.3.33ns) & frt -Wv,-r128 -Of -KA32 & 1156 & 8784 & 9600 \\
\hline IBM eServer pSeries 6556514 proc(1300 MHz) & & & 10880 & 20800 \\
\hline IBM eServer pSeries $6556511 \operatorname{proc}(1300 \mathrm{MHz})$ & $\begin{array}{l}\text {-O3 -qarch=pwr4 -qtune=pwr4 -Pv } \\
-\mathrm{Wp},- \text { ea478,-g1 }\end{array}$ & 1135 & 2899 & 5200 \\
\hline Cray T932 (32 proc. 2.2 ns) & & & 29360 & 57600 \\
\hline
\end{tabular}




\begin{tabular}{|c|c|c|c|c|}
\hline Computer & $\begin{array}{c}\text { "LINPACK Benchmark" } \\
\text { OS/Compiler }\end{array}$ & $\begin{array}{c}n=100 \\
\text { Mflop/s }\end{array}$ & $\begin{array}{c}\text { "TPP" } \\
\text { Best } \\
\text { Effort } \\
\text { n=1000 } \\
\text { Mflop/s }\end{array}$ & $\begin{array}{l}\text { "Theoritical } \\
\text { Peak" } \\
\text { Mflop/s }\end{array}$ \\
\hline Cray T928 (28 proc. $2.2 \mathrm{~ns})$ & & & 28340 & 50400 \\
\hline Cray T924 (24 proc. $2.2 \mathrm{~ns})$ & & & 26170 & 43200 \\
\hline Cray T916 (16 proc. $2.2 \mathrm{~ns})$ & & & 19980 & 28800 \\
\hline Cray T916 (8 proc. $2.2 \mathrm{~ns})$ & & & 10880 & 14400 \\
\hline Cray T94 (4 proc. 2.2 ns) & f90-O3, inline 2 & 1129 & 5735 & 7200 \\
\hline HP AlphaServer GS1280 7/1300 (8 proc $1.3 \mathrm{GHz}$ ) & & & 14260 & 20800 \\
\hline HP AlphaServer GS1280 7/1300 (4 proc $1.3 \mathrm{GHz}$ ) & & & 7781 & 10400 \\
\hline HP AlphaServer GS1280 7/1300 (2 proc $1.3 \mathrm{GHz}$ ) & & & 3890 & 5200 \\
\hline HP AlphaServer GS1280 7/1300 (1 proc $1.3 \mathrm{GHz})$ & 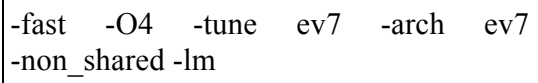 & 1122 & 2132 & 2600 \\
\hline hp rx5670 Itanium $2(4$ proc $1 \mathrm{GHz})$ & & & 11430 & 16000 \\
\hline hp rx5670 Itanium $2(2$ proc $1 \mathrm{GHz})$ & & & 6284 & 8000 \\
\hline hp rx5670 Itanium $2(1$ proc $1 \mathrm{GHz})$ & $\begin{array}{l}\text { f90 +DSmckinley }+\mathrm{O} 3 \\
\text { +Oinline_budget }=100000 \\
\text { +Ono_ptrs_to_globals }\end{array}$ & 1102 & 3534 & 4000 \\
\hline hp rx2600 Itanium $2(2$ proc $1 \mathrm{GHz})$ & & & 6251 & 8000 \\
\hline hp rx2600 Itanium $2(1$ proc $1 \mathrm{GHz})$ & $\begin{array}{l}\text { f90 +DSmckinley +O3 } \\
\text { +Oinline_budget }=100000 \\
\text { +Ono_ptrs_to_globals }\end{array}$ & 1102 & 3528 & 4000 \\
\hline hp zx6000 Itanium $2(2$ proc $1 \mathrm{GHz})$ & & & 6315 & 8000 \\
\hline hp zx6000 Itanium $2(1$ proc $1 \mathrm{GHz})$ & $\begin{array}{l}\text { f90 +DSmckinley }+\mathrm{O} 3 \\
\text { +Oinline_budget }=100000 \\
\text { +Ono_ptrs_to_globals }\end{array}$ & 1102 & 3533 & 4000 \\
\hline $\begin{array}{l}\text { IBM eServer pSeries } 690 \text { Turbo } 16 \text { proc }(1300 \\
\text { MHz) }\end{array}$ & & & 28080 & 83200 \\
\hline IBM eServer pSeries 690 Turbo 8 proc(1300 MHz) & & & 18290 & 41600 \\
\hline IBM eServer pSeries 690 Turbo 1 proc(1300 MHz) & $\begin{array}{l}\text {-O3 -qarch=pwr4 -qtune=pwr4 -Pv } \\
-\mathrm{Wp},- \text { ea478,-g1 }\end{array}$ & 1074 & 2894 & 5200 \\
\hline Intel Xeon $2.4 \mathrm{GHz}$ & ifort -ipo & 1055 & & 4800 \\
\hline Intel P4 $2200 \mathrm{MHz}$ & ifc -O3 -xW -align -ipo -Zp16 -r8 & 1033 & 1911 & 4400 \\
\hline IBM eServer pSeries $6156 \mathrm{C} 3(1$ proc $1.2 \mathrm{GHz} 4+)$ & $\begin{array}{l}\text {-O3 -qarch=pwr4 -qtune=pwr4 -Pv } \\
\text {-Wp,-ea478,-g1 }\end{array}$ & 1032 & & 4800 \\
\hline IBM eServer pSeries $6156 \mathrm{E} 3(1$ proc $1.2 \mathrm{GHz} \mathrm{P} 4+)$ & $\begin{array}{l}\text {-O3 -qarch=pwr4 -qtune=pwr4 -Pv } \\
-\mathrm{Wp},- \text { ea478,-g1 }\end{array}$ & 1032 & & 4800 \\
\hline hp AlphaServer ES45 68/1250(4 proc) & & & 7132 & 10000 \\
\hline hp AlphaServer ES45 68/1250(2 proc) & & & 3721 & 5000 \\
\hline hp AlphaServer ES45 68/1250(1 proc) & v5.5-1877-O4 & 1031 & 1945 & 2500 \\
\hline Cray T94 (3 proc. $2.2 \mathrm{~ns})$ & f90-O3, inline2 & 1029 & 4387 & 5400 \\
\hline IBM eServer pSeries $6306 \mathrm{C} 4$ (4 proc $1.2 \mathrm{GHz} \mathrm{P} 4+$ ) & & & 9255 & 19200 \\
\hline IBM eServer pSeries $6306 \mathrm{C} 4(1$ proc $1.2 \mathrm{GHz}$ p $4+)$ & $\begin{array}{l}\text {-O3 -qarch=pwr4 -qtune=pwr4 -Pv } \\
-\mathrm{Wp},- \text { ea478,-g1 }\end{array}$ & 1025 & 2727 & 4800 \\
\hline IBM eServer pSeries 630 6E4 (4 proc $1.2 \mathrm{GHz} \mathrm{P} 4+$ ) & & & 9255 & 19200 \\
\hline
\end{tabular}




\begin{tabular}{|c|c|c|c|c|}
\hline Computer & $\begin{array}{c}\text { "LINPACK Benchmark" } \\
\text { OS/Compiler }\end{array}$ & $\begin{array}{c}\mathbf{n}=100 \\
\text { Mflop/s }\end{array}$ & $\begin{array}{c}\text { "TPP" } \\
\text { Best } \\
\text { Effort } \\
\text { n=1000 } \\
\text { Mflop/s }\end{array}$ & $\begin{array}{l}\text { "Theoritical } \\
\text { Peak" } \\
\text { Mflop/s }\end{array}$ \\
\hline IBM eServer pSeries $6306 \mathrm{E} 4(1$ proc $1.2 \mathrm{GHz}$ p $4+)$ & $\begin{array}{l}\text {-O3 -qarch=pwr4 -qtune=pwr4 -Pv } \\
-\mathrm{Wp},- \text { ea478,-g1 }\end{array}$ & 1025 & 2727 & 4800 \\
\hline Intel xeon 64 (dual 3.6 GHz) & icf -O3 & 1010 & & 7200 \\
\hline HP AlphaServer ES80 7/1150 (8 proc $1.15 \mathrm{GHz})$ & & & 11410 & 18400 \\
\hline HP AlphaServer ES80 7/1150 (4 proc $1.15 \mathrm{GHz})$ & & & 6584 & 9200 \\
\hline HP AlphaServer ES80 7/1150 (2 proc $1.15 \mathrm{GHz})$ & & & 3424 & 4600 \\
\hline HP AlphaServer ES80 7/1150 (1 proc $1.15 \mathrm{GHz})$ & $\begin{array}{l}\text {-fast -O4 -tune ev7 -arch ev7 } \\
\text {-non_shared -lm }\end{array}$ & 992 & 1884 & 2300 \\
\hline HP AlphaServer ES47 7/1150 (4 proc $1.15 \mathrm{GHz})$ & & & 6584 & 9200 \\
\hline HP AlphaServer ES47 7/1150 (2 proc $1.15 \mathrm{GHz})$ & & & 3424 & 4600 \\
\hline HP AlphaServer ES47 7/1150 (1 proc $1.15 \mathrm{GHz})$ & $\begin{array}{l}\text {-fast -O4 -tune ev7 -arch ev7 } \\
\text {-non_shared -lm }\end{array}$ & 992 & 1884 & 2300 \\
\hline hp zx2000 Itanium 2(900MHz) & $\begin{array}{l}\text { f90 +DSmckinley }+\mathrm{O} 3 \\
+ \text { Oinline_budget }=100000 \\
\text { +Ono_ptrs_to_globals }\end{array}$ & 992 & 3081 & 3600 \\
\hline Fujitsu Siemens hpcLine(Xeon 2GHz) & fc -O3 -align -r8 -ipo - $x W$ & 969 & 1648 & 2000 \\
\hline AMD Opteron 242/1.6 Ghz (2 proc) & & & 3377 & 6400 \\
\hline AMD Opteron $242 / 1.6 \mathrm{Ghz}$ (1 proc) & ifort -xW -ipo -O3 & 988 & 1808 & 3200 \\
\hline Cray SV1ex-1-32(31 proc,500MHz) & & & 15520 & 62000 \\
\hline Cray SV1ex-1-32(28 proc,500MHz) & & & 15250 & 56000 \\
\hline Cray SV1ex-1-32(24 proc,500MHz) & & & 14750 & 48000 \\
\hline Cray SV1ex-1-32(20 proc,500MHz) & & & 14150 & 40000 \\
\hline Cray SV1ex-1-32(16 proc,500MHz) & & & 13050 & 32000 \\
\hline Cray SV1ex-1-32(12 proc,500MHz) & f90 -O3, inline2 & 988 & 11250 & 24000 \\
\hline $\begin{array}{l}\text { HCL Infiniti Global line } 4700 \mathrm{HW} \text { (Intel Xeon } 3.16 \\
\mathrm{GHz} \text { ) }\end{array}$ & ifort -fast -r8 -align & 981 & 3209 & 6320 \\
\hline Cray T94 (2 proc. $2.2 \mathrm{~ns})$ & f90 -O3, inline2 & 962 & 2998 & 3600 \\
\hline IBM eServer pSeries $6556518 \operatorname{proc}(1100 \mathrm{MHz})$ & & & 16170 & 35200 \\
\hline IBM eServer pSeries $6556511 \operatorname{proc}(1100 \mathrm{MHz})$ & $\begin{array}{l}-\mathrm{O} 3 \text {-qarch=pwr4 -qtune=pwr4 -Pv } \\
-\mathrm{Wp},- \text { ea478,-g1 }\end{array}$ & 937 & 2484 & 4400 \\
\hline Cray SV1ex-1-32(8 proc,500MHz) & & & 8938 & 16000 \\
\hline Cray SV1ex-1-32(4 proc,500MHz) & & & 5358 & 8000 \\
\hline Cray SV1ex-1-32(2 proc,500MHz) & & & 2947 & 4000 \\
\hline Cray SV1ex-1-32(1 proc,500MHz) & f90 -O3, inline2 & 935 & 1554 & 2000 \\
\hline hp GS1280 7/1150 (4 proc, $1.15 \mathrm{GHz})$ & & & 6584 & 9200 \\
\hline hp GS1280 7/1150 (2 proc,1.15 GHz) & & & 3493 & 4600 \\
\hline hp GS1280 7/1150 (1 proc,1.15 GHz) & KAP -O4 & 914 & 1879 & 2300 \\
\hline AMD Opteron 242/1.6 Ghz (2 proc) & & & 4370 & 6400 \\
\hline Intel Xeon $2.4 \mathrm{GHz}$ & ifort -O3 & 884 & & 4800 \\
\hline AMD Opteron 242/1.6 Ghz (1 proc) & pgf77 -fast -tp k8-64 & 882 & 2325 & 3200 \\
\hline Dell PowerEdge 1850s (3.2 GHz Intel & Intel 9 FORTRAN & 873 & 2800 & 6400 \\
\hline
\end{tabular}




\begin{tabular}{|c|c|c|c|c|}
\hline Computer & $\begin{array}{c}\text { "LINPACK Benchmark" } \\
\text { OS/Compiler }\end{array}$ & $\begin{array}{c}\mathrm{n}=\mathbf{1 0 0} \\
\text { Mflop/s }\end{array}$ & $\begin{array}{c}\text { "TPP" } \\
\text { Best } \\
\text { Effort } \\
\text { n=1000 } \\
\text { Mflop/s }\end{array}$ & $\begin{array}{l}\text { "Theoritical } \\
\text { Peak" } \\
\text { Mflop/s }\end{array}$ \\
\hline \multicolumn{5}{|l|}{ EM64T) } \\
\hline $\begin{array}{l}\text { IBM IntelliStation POWER } 2751 \mathrm{CPU}(1000 \\
\text { MHz POWER4+) }\end{array}$ & $\begin{array}{l}\text {-O3 -qarch=pwr4 -qtune=pwr4 -Pv } \\
\text {-Wp,-ea478,-g1 }\end{array}$ & 860 & 2327 & 4000 \\
\hline NEC SX-5/1 (1 proc. $4.0 \mathrm{~ns})$ & R9.1 -pi -wf"-prob_use" & 856 & 7280 & 8000 \\
\hline HP 9000 rp8420-32 (1000MHz PA-8800), 8 proc & & & 14150 & 32000 \\
\hline HP 9000 rp8420-32 (1000MHz PA-8800), 4 proc & & & 9478 & 16000 \\
\hline HP 9000 rp8420-32 (1000MHz PA-8800), 2 proc & & & 5435 & 8000 \\
\hline HP 9000 rp8420-32 (1000MHz PA-8800), 1 proc & $\begin{array}{l}\text { HP-UX 11i, HP f90 } 11.11 .74 \text { f90 +O3 } \\
\text { +Onolimit +Onodataprefetch } \\
\text { +Oinline_budget }=1000000 \\
\text { +Oloop_unroll=6 }\end{array}$ & 843 & 2905 & 4000 \\
\hline HP 9000 Superdome (1000MHz PA-8800), 8 proc & & & 14070 & 32000 \\
\hline HP 9000 Superdome (1000MHz PA-8800), 4 proc & & & 9260 & 16000 \\
\hline HP 9000 Superdome (1000MHz PA-8800), 2 proc & & & 5432 & 8000 \\
\hline HP 9000 Superdome (1000MHz PA-8800), 1 proc & $\begin{array}{l}\text { HP-UX 11i, HP f90 } 11.11 .74 \text { f90 +O3 } \\
\text { +Onolimit +Onodataprefetch } \\
\text { +Oinline_budget }=1000000 \\
\text { +Oloop_unroll=6 }\end{array}$ & 843 & 2902 & 4000 \\
\hline IBM eServer pSeries $6306 \mathrm{C} 44 \operatorname{proc}(1000 \mathrm{MHz})$ & $\begin{array}{l}-\mathrm{O} 3 \text {-qarch=pwr4 -qtune=pwr4 -Pv } \\
-\mathrm{Wp},- \text { ea478,-g1 }\end{array}$ & & 6769 & 16000 \\
\hline IBM eServer pSeries $6306 \mathrm{C} 41 \operatorname{proc}(1000 \mathrm{MHz})$ & $\begin{array}{l}-\mathrm{O} 3 \text {-qarch=pwr4 -qtune=pwr4 -Pv } \\
-\mathrm{Wp},- \text { ea478,-g1 }\end{array}$ & 842 & 2173 & 4000 \\
\hline IBM eServer pSeries $6306 \mathrm{E} 44 \operatorname{proc}(1000 \mathrm{MHz})$ & $\begin{array}{l}-\mathrm{O} 3 \text {-qarch=pwr4 -qtune=pwr4 -Pv } \\
-\mathrm{Wp},-\mathrm{ea} 478,-\mathrm{g} 1\end{array}$ & & 6769 & 16000 \\
\hline IBM eServer pSeries $6306 \mathrm{E} 41 \operatorname{proc}(1000 \mathrm{MHz})$ & $\begin{array}{l}-\mathrm{O} 3 \text {-qarch=pwr4 -qtune=pwr4 -Pv } \\
-\mathrm{Wp},- \text { ea478,-g1 }\end{array}$ & 842 & 2173 & 4000 \\
\hline AMD Athlon MP1800+(1 proc $1530 \mathrm{MHz})$ & ifc -O3 -tpp6 -ipo & 832 & 1705 & 3060 \\
\hline Compaq ES45 (4 proc. $1000 \mathrm{MHz}$ ) & & & 5522 & 8000 \\
\hline Compaq ES45 (3 proc. $1000 \mathrm{MHz}$ ) & & & 4076 & 6000 \\
\hline Compaq ES45 (2 proc. $1000 \mathrm{MHz})$ & & & 2901 & 4000 \\
\hline Compaq ES45 (1 proc. $1000 \mathrm{MHz})$ & $\begin{array}{l}\text { kf77 }- \text { fkapargs }=\text {-inline }=\text { daxpy }- \text { ur }=8 \\
\text {-ur2=320' -arch host -assume } \\
\text { nounderscore }\end{array}$ & 824 & 1542 & 2000 \\
\hline NEC SX-5/16 (16 proc. $4.0 \mathrm{~ns})$ & & & 45030 & 64000 \\
\hline NEC SX-5/8 (8 proc. $4.0 \mathrm{~ns})$ & & & 32570 & 64000 \\
\hline NEC SX-5/4 (4 proc. $4.0 \mathrm{~ns})$ & & & 19220 & 32000 \\
\hline NEC SX-5/2 (2 proc. $4.0 \mathrm{~ns})$ & & & 11150 & 16000 \\
\hline Fujitsu VPP800/1 (1 proc 4.0ns) & frt -Wv,-r128 -Of -KA32 & 813 & 7091 & 8000 \\
\hline Intel P4 $1700 \mathrm{MHz}$ & ifc -O3 -xW -align -r8 -ipo & 796 & & 3400 \\
\hline hp ES80 7/1000(4 proc, $1 \mathrm{GHz})$ & & & 5706 & 8000 \\
\hline hp ES80 7/1000(2 proc, $1 \mathrm{GHz})$ & & & 3003 & 4000 \\
\hline hp ES80 7/1000(1 proc, $1 \mathrm{GHz})$ & KAP -O4 & 790 & 1635 & 2000 \\
\hline
\end{tabular}




\begin{tabular}{|c|c|c|c|c|}
\hline Computer & $\begin{array}{c}\text { "LINPACK Benchmark" } \\
\text { OS/Compiler }\end{array}$ & $\begin{array}{c}\mathrm{n}=\mathbf{1 0 0} \\
\mathrm{Mflop} / \mathrm{s}\end{array}$ & $\begin{array}{c}\text { “TPP" } \\
\text { Best } \\
\text { Effort } \\
\text { n=1000 } \\
\text { Mflop/s }\end{array}$ & $\begin{array}{l}\text { "Theoritical } \\
\text { Peak" } \\
\text { Mflop/s }\end{array}$ \\
\hline hp ES47 7/1000(4 proc, $1 \mathrm{GHz})$ & & & 5706 & 8000 \\
\hline hp ES47 7/1000(2 proc, $1 \mathrm{GHz})$ & & & 3003 & 4000 \\
\hline hp ES47 7/1000(1 proc, $1 \mathrm{GHz})$ & KAP -O4 & 790 & 1635 & 2000 \\
\hline HP Superdome (16 proc $875 \mathrm{MHz}$ ) & & & 19210 & 56000 \\
\hline HP Superdome ( 8 proc $875 \mathrm{MHz}$ ) & & & 12370 & 28000 \\
\hline HP Superdome ( 4 proc $875 \mathrm{MHz}$ ) & & & 7257 & 14000 \\
\hline HP Superdome ( 2 proc $875 \mathrm{MHz}$ ) & & & 4046 & 7000 \\
\hline AMD Opteron $1.4 \mathrm{GHz}$ & -O3 -tpp7 -axK -ipo -align -r8 & 781 & 2020 & 3800 \\
\hline HP Superdome ( 1 proc $875 \mathrm{MHz}$ ) & $\begin{array}{l}\mathrm{f} 90+\mathrm{O} 3+\text { Oinlinebudget }=1000000 \\
+ \text { Onodataprefetch }+ \text { Oloop_unroll }=6\end{array}$ & 769 & 2305 & 3500 \\
\hline hp server rp8400(16 proc $875 \mathrm{MHz})$ & & & 17750 & 56000 \\
\hline hp server rp $8400(8$ proc $875 \mathrm{MHz})$ & & & 11710 & 28000 \\
\hline hp server rp8400(4 proc $875 \mathrm{MHz})$ & & & 7096 & 14000 \\
\hline hp server rp8400(2 proc $875 \mathrm{MHz})$ & & & 4033 & 7000 \\
\hline hp server rp8400(1 proc $875 \mathrm{MHz})$ & $\begin{array}{l}\mathrm{f} 90+\mathrm{O} 3+\text { Oinlinebudget }=1000000 \\
+ \text { Onodataprefetch }+ \text { Oloop_unroll }=6\end{array}$ & 769 & 2320 & 3500 \\
\hline hp server rp $7410(8$ proc $875 \mathrm{MHz})$ & & & 12900 & 28000 \\
\hline hp server rp7410(4 proc $875 \mathrm{MHz})$ & & & 7507 & 14000 \\
\hline hp server rp7410(2 proc $875 \mathrm{MHz})$ & & & 4179 & 7000 \\
\hline hp server rp $7410(1$ proc $875 \mathrm{MHz})$ & $\begin{array}{l}\mathrm{f} 90+\mathrm{O} 3+\text { Oinlinebudget }=1000000 \\
+ \text { Onodataprefetch }+ \text { Oloop_unroll }=6\end{array}$ & 769 & 2337 & 3500 \\
\hline Cray SV1-1-32 (31 proc. $300 \mathrm{MHz})$ & & & 10910 & 37200 \\
\hline Cray SV1-1-32 (28 proc. $300 \mathrm{MHz})$ & & & 10770 & 33600 \\
\hline Cray SV1-1-32 (24 proc. $300 \mathrm{MHz})$ & & & 10420 & 28800 \\
\hline Cray SV1-1-32 (20 proc. $300 \mathrm{MHz})$ & & & 9945 & 24000 \\
\hline Cray SV1-1-32 (16 proc. $300 \mathrm{MHz})$ & f90 -O3, inline2 & 751 & 9156 & 19200 \\
\hline Cray SV1-1-32 (12 proc. $300 \mathrm{MHz})$ & f90 -O3, inline2 & 748 & 7837 & 14000 \\
\hline Tyan S2460/AMD Athlon XP(1533 MHz,2 proc) & & & 2176 & 6132 \\
\hline Tyan S2460/AMD Athlon XP(1533 MHz,1 proc) & ifc -tpp6-O3 & 732 & 1623 & 3066 \\
\hline Intel P4 ACER(Veriton 7200)1700 MHz & ifc -O3 -xW -align -r8 -ipo & 712 & & 3400 \\
\hline Cray SV1-1-32 (8 proc. $300 \mathrm{MHz})$ & f90 -O3, inline2 & 710 & 6055 & 9600 \\
\hline Cray T94 (1 proc. 2.2 ns) & f90 -O3, inline2 & 705 & 1603 & 1800 \\
\hline AMD Athlon Thunderbird $1.4 \mathrm{GHz}$ & ifc -O3 -tpp6 -align -r8 -ipo & 704 & & 2800 \\
\hline Compaq DS20L 833 MHz (2 proc.) & & & 2316 & 3332 \\
\hline Compaq DS20L $833 \mathrm{MHz}$ & $\begin{array}{l}\text { kf77 }- \text { fkapargs }=\text {-inline }=\text { daxpy }- \text { ur }=8 \\
\text {-ur2=320' -arch host -assume } \\
\text { nounderscore }\end{array}$ & 699 & 1232 & 1666 \\
\hline Fujitsu Siemens Celsius 460 (P4, $1.5 \mathrm{GHz})$ & Intel fortran90 -O3 - $\mathrm{xW}$-align -r8 & 675 & 955 & 1500 \\
\hline
\end{tabular}




\begin{tabular}{|c|c|c|c|c|}
\hline Computer & $\begin{array}{c}\text { "LINPACK Benchmark" } \\
\text { OS/Compiler }\end{array}$ & $\begin{array}{c}n=100 \\
\text { Mflop/s }\end{array}$ & $\begin{array}{c}\text { "TPP" } \\
\text { Best } \\
\text { Effort } \\
\text { n=1000 } \\
\text { Mflop/s }\end{array}$ & $\begin{array}{l}\text { "Theoritical } \\
\text { Peak" } \\
\text { Mflop/s }\end{array}$ \\
\hline HP SuperDome (16 proc $750 \mathrm{MHz}$ ) & & & 17660 & 48000 \\
\hline HP SuperDome ( 8 proc $750 \mathrm{MHz}$ ) & & & 11260 & 24000 \\
\hline HP SuperDome ( 4 proc $750 \mathrm{MHz}$ ) & & & 6667 & 12000 \\
\hline HP SuperDome ( 2 proc $750 \mathrm{MHz}$ ) & & & 3711 & 6000 \\
\hline HP SuperDome ( 1 proc $750 \mathrm{MHz}$ ) & $\begin{array}{l}\mathrm{f} 90+\mathrm{O} 3+\text { Oinlinebudget }=1000000 \\
+ \text { Onodataprefetch }+ \text { Oloop_unroll }=6\end{array}$ & 669 & 2099 & 3000 \\
\hline hp server rp8400(16proc $750 \mathrm{MHz})$ & & & 16500 & 48000 \\
\hline hp server rp8400(8 proc $750 \mathrm{MHz})$ & & & 10810 & 24000 \\
\hline hp server rp8400(4 proc $750 \mathrm{MHz})$ & & & 6522 & 12000 \\
\hline hp server rp8400(2 proc $750 \mathrm{MHz})$ & & & 3681 & 6000 \\
\hline hp server rp8400(1 proc $750 \mathrm{MHz})$ & $\begin{array}{l}990+\mathrm{O} 3+\text { Oinlinebudget }=1000000 \\
+ \text { Onodataprefetch }+ \text { Oloop_unroll }=6\end{array}$ & 669 & 2099 & 3000 \\
\hline hp server rp $7400(8$ proc $750 \mathrm{MHz})$ & & & 10550 & 24000 \\
\hline hp server rp7400(4 proc $750 \mathrm{MHz})$ & & & 6667 & 12000 \\
\hline hp server rp $7400(2$ proc $750 \mathrm{MHz})$ & & & 3681 & 6000 \\
\hline hp server rp7400(1 proc $750 \mathrm{MHz})$ & $\begin{array}{l}\mathrm{f} 90+\mathrm{O} 3+\text { Oinlinebudget }=1000000 \\
+ \text { Onodataprefetch }+ \text { Oloop_unroll }=6\end{array}$ & 669 & 2085 & 3000 \\
\hline AMD ATHLON Thunderbird $1.2 \mathrm{GHz}$ & ifc -tpp7 -O3 -ipo & 649 & 1402 & 2400 \\
\hline Compaq ES40 (833 MHz 4cpu) & & & 4626 & 6664 \\
\hline Compaq ES40 (833 MHz 2cpu) & & & 2411 & 3332 \\
\hline Compaq ES40 (833 MHz 1cpu) & -assume nounderscore -O5 & 639 & 1277 & 1666 \\
\hline Dell PE7150 Itanium(800Mhz 4 proc) & & & 7358 & 12800 \\
\hline Dell PE7150 Itanium(800Mhz 2 proc) & & & 4504 & 6400 \\
\hline Dell PE7150 Itanium(800Mhz 1 proc) & efl -Ox -Ob2 -Ot & 600 & 2382 & 3200 \\
\hline Cray T3E-1350 (16 proc $675 \mathrm{MHz})$ & & & 3204 & 24000 \\
\hline Cray T3E-1350 (12 proc $675 \mathrm{MHz})$ & & & 2716 & 18000 \\
\hline Cray T3E-1350 (8 proc $675 \mathrm{MHz})$ & & & 2518 & 12000 \\
\hline Cray T3E-1350 (6 proc $675 \mathrm{MHz})$ & & & 2199 & 9000 \\
\hline Cray T3E-1350 (4 proc $675 \mathrm{MHz})$ & & & 1797 & 6000 \\
\hline Cray T3E-1350 (2 proc $675 \mathrm{MHz})$ & & & 1197 & 3000 \\
\hline Cray T3E-1350 (1 proc $675 \mathrm{MHz})$ & f90 ver. 3.5 -O3, inline2 & 591 & 728 & 1500 \\
\hline Cray SV1-1-32 (4 proc. $300 \mathrm{MHz}$ ) & f90 -O3, inline2 & 596 & 3574 & 4800 \\
\hline Intel/HP Itanium $800 \mathrm{MHz}$ & f90 +Ofast $+\mathrm{O} 3+$ Onodataprefetch & 580 & 2282 & 3200 \\
\hline HP i2000 Itanium $800 \mathrm{MHz}(2$ proc) & & & 3888 & 6400 \\
\hline HP i2000 Itanium $800 \mathrm{MHz}(1$ proc $)$ & f90 +Ofast $+\mathrm{O} 3+$ Onodatapretch & 580 & 2282 & 3200 \\
\hline NEC SX-4/32 (32 proc. $8.0 \mathrm{ns)}$ & & & 31060 & 64000 \\
\hline NEC SX-4/24 (24 proc. $8.0 \mathrm{ns)}$ & & & 27440 & 48000 \\
\hline
\end{tabular}




\begin{tabular}{|c|c|c|c|c|}
\hline Computer & $\begin{array}{c}\text { "LINPACK Benchmark" } \\
\text { OS/Compiler }\end{array}$ & $\begin{array}{c}\mathbf{n}=100 \\
\text { Mflop/s }\end{array}$ & $\begin{array}{c}\text { "TPP" } \\
\text { Best } \\
\text { Effort } \\
\text { n=1000 } \\
\text { Mflop/s }\end{array}$ & $\begin{array}{l}\text { "Theoritical } \\
\text { Peak" } \\
\text { Mflop/s }\end{array}$ \\
\hline NEC SX-4/16 (16 proc. $8.0 \mathrm{~ns})$ & & & 21470 & 32000 \\
\hline NEC SX-4/8 (8 proc. $8.0 \mathrm{~ns})$ & & & 12780 & 16000 \\
\hline NEC SX-4/4 (4 proc. $8.0 \mathrm{~ns})$ & & & 6780 & 8000 \\
\hline NEC SX-4/2 (2 proc. $8.0 \mathrm{~ns})$ & & & 3570 & 4000 \\
\hline NEC SX-4/1 (1 proc. $8.0 \mathrm{~ns})$ & 137 R6.1 -fopp $f=x$ inline & 578 & 1944 & 2000 \\
\hline AMD Athlon Thunderbird $1200 \mathrm{Mhz}$ & $\begin{array}{l}\text { g77 -s -static -O3 -fomit-frame-pointer } \\
\text {-Wall -mpentiumpro -march=pentiumpro } \\
\text {-malign-functions=4 -funroll-loops } \\
\text {-fexpensive-optimizations } \\
\text {-malign-double -fschedule-insns2 } \\
\text {-mwide-multiply }\end{array}$ & 558 & 1029 & 2400 \\
\hline Compaq Server DS20e(2 proc $667 \mathrm{MHz})$ & & & 1923 & 2668 \\
\hline Compaq Server DS20e(667 MHz) & & 558 & 1025 & 1334 \\
\hline Compaq Server ES40(4 proc 667MHz) & & & 3804 & 5336 \\
\hline Compaq Server ES40(2 proc $667 \mathrm{MHz})$ & & & 1923 & 2668 \\
\hline Compaq Server ES40(1 proc $667 \mathrm{MHz})$ & $\begin{array}{l}\text { kf77 }- \text { fkapargs }=\text { '-inline }=\text { daxpy }- \text { ur }=12 \\
\text {-ur2=320' -O5 -tune ev5 -assume } \\
\text { nounderscore }\end{array}$ & 561 & 1031 & 1334 \\
\hline Cray SV1-1-32 (2 proc. $300 \mathrm{MHz})$ & & & 1959 & 2400 \\
\hline Cray SV1-1-32 (1 proc. $300 \mathrm{MHz}$ ) & f90-O3, inline 2 & 549 & 1028 & 1200 \\
\hline NEC SX-4B/2(2proc.8.8ns) & & & 3246 & 3636 \\
\hline NEC SX-4B/1(1proc. $8.8 \mathrm{~ns})$ & R7.1 -fopp $\mathrm{f}=\mathrm{x}$ inline & 524 & 1767 & 1818 \\
\hline Tyan S2518/PentiumIII(1266 MHz,2 proc) & & & 1478 & 2532 \\
\hline Tyan S2518/PentiumIII(1266 MHz,1 proc) & ifc -tpp6 -O3 & 503 & 830 & 1266 \\
\hline IBM RS/6000 44P-270 4 proc (450MHz,8MBL2) & & & 4396 & 7200 \\
\hline IBM RS/6000 44P-270 2 proc (450MHz,8MBL2) & & & 2521 & 3600 \\
\hline IBM RS/6000 44P-270 1 proc(450MHz,8MBL2) & $\begin{array}{l}\text {-O -Q -qfloat=hsflt -qarch=pwr3 } \\
\text {-qtune }=\text { pwr3 -Pv -Wp,-ea478,-g1 }\end{array}$ & 503 & 1451 & 1800 \\
\hline $\begin{array}{l}\text { IBM eServer pSeries } 610 \text { Model B } 804 \operatorname{proc}(450 \\
\text { MHz 8MB L2) }\end{array}$ & & & 4396 & 7200 \\
\hline $\begin{array}{l}\text { IBM eServer pSeries } 610 \text { Model B } 802 \operatorname{proc}(450 \\
\text { MHz 8MB L2) }\end{array}$ & & & 2521 & 3600 \\
\hline $\begin{array}{l}\text { IBM eServer pSeries } 610 \text { Model B } 801 \operatorname{proc}(450 \\
\text { MHz 8MB L2) }\end{array}$ & $\begin{array}{l}\text {-O -Q -qfloat=hsflt -qarch=pwr3 } \\
\text {-qtune }=\text { pwr3 -Pv -Wp,-ea478,-g1 }\end{array}$ & 503 & 1451 & 1800 \\
\hline $\begin{array}{l}\text { IBM eServer pSeries } 610 \text { Model 6E1 } 2 \text { proc( } 450 \\
\text { MHz 8MB L2) }\end{array}$ & & & 2521 & 3600 \\
\hline $\begin{array}{l}\text { IBM eServer pSeries } 610 \text { Model } 6 \mathrm{E} 11 \operatorname{proc}(450 \\
\text { MHz 8MB L2) }\end{array}$ & $\begin{array}{l}\text {-O -Q -qfloat=hsflt -qarch=pwr3 } \\
\text {-qtune }=\text { pwr3 -Pv -Wp,-ea478,-g1 }\end{array}$ & 503 & 1451 & 1800 \\
\hline $\begin{array}{l}\text { IBM eServer pSeries } 610 \text { Model 6C1 } 2 \operatorname{proc}(450 \\
\text { MHz 8MB L2) }\end{array}$ & & & 2521 & 3600 \\
\hline $\begin{array}{l}\text { IBM eServer pSeries } 610 \text { Model 6C1 } 1 \text { proc(450 } \\
\text { MHz 8MB L2) }\end{array}$ & $\begin{array}{l}\text {-O -Q -qfloat=hsflt -qarch=pwr3 } \\
\text {-qtune=pwr3 -Pv -Wp,-ea478,-g1 }\end{array}$ & 503 & 1451 & 1800 \\
\hline IBM RS/6000 44P-170 (450 MHz) & $\begin{array}{l}\text {-O -Q -qfloat=hsflt -qarch=pwr3 } \\
\text {-qtune=pwr3 -Pv -Wp,-ea478,-g1 }\end{array}$ & 503 & 1440 & 1800 \\
\hline
\end{tabular}




\begin{tabular}{|c|c|c|c|c|}
\hline Computer & $\begin{array}{c}\text { "LINPACK Benchmark" } \\
\text { OS/Compiler }\end{array}$ & $\begin{array}{c}n=100 \\
\text { Mflop/s }\end{array}$ & $\begin{array}{c}\text { "TPP" } \\
\text { Best } \\
\text { Effort } \\
\text { n=1000 } \\
\text { Mflop/s }\end{array}$ & $\begin{array}{l}\text { "Theoritical } \\
\text { Peak" } \\
\text { Mflop/s }\end{array}$ \\
\hline NEC SX-4/Ce (1 proc. ) & R7.1 -fopp $\mathrm{f}=\mathrm{x}$ inline & 500 & 980 & 1000 \\
\hline AMD Duron $900(900 \mathrm{MHz})$ & ifc -static -O3 -tpp6 -ipo & 486 & 977 & 1800 \\
\hline Fujitsu Siemens Celsius 460 (P4, $1.5 \mathrm{GHz})$ & pgf90 -fast & 483 & 955 & 1500 \\
\hline Cray C90 (16 proc. $4.2 \mathrm{~ns})$ & CF77 $5.0-Z p-W d-e 68$ & 479 & 10780 & 15238 \\
\hline HP SuperDome (16 proc $552 \mathrm{MHz}$ ) & & & 12220 & 35328 \\
\hline HP SuperDome (8 proc $552 \mathrm{MHz}$ ) & & & 8055 & 17664 \\
\hline HP SuperDome (4 proc $552 \mathrm{MHz}$ ) & & & 4319 & 8832 \\
\hline HP SuperDome (2 proc $552 \mathrm{MHz}$ ) & & & 2506 & 4416 \\
\hline HP SuperDome (1 proc $552 \mathrm{MHz}$ ) & $\mathrm{f} 77+\mathrm{O} 3+$ Oinline $=$ daxpy & 470 & 1497 & 2208 \\
\hline Cray C90 (8 proc. $4.2 \mathrm{~ns})$ & CF77 $5.0-Z p-W d-e 68$ & 468 & 6175 & 7619 \\
\hline HP N4000 (8 proc. $550 \mathrm{MHz}$ ) & & & 7762 & 17600 \\
\hline HP N4000 (4 proc. $550 \mathrm{MHz}$ ) & & & 4494 & 8800 \\
\hline HP N4000 (2 proc. $550 \mathrm{MHz}$ ) & & & 2662 & 4400 \\
\hline HP N4000 (1 proc. $550 \mathrm{MHz})$ & $\mathrm{f} 77+\mathrm{O} 3+$ Oinline $=$ daxpy & 468 & 1583 & 2200 \\
\hline NEC SX-4/16A(16proc.8.0ns) & & & 20620 & 32000 \\
\hline NEC SX-4/8A(8proc.8.0ns) & & & 12490 & 16000 \\
\hline NEC SX-4/4A(4proc.8.0ns) & & & 6692 & 8000 \\
\hline NEC SX-4/2A(2proc.8.0ns) & & & 3525 & 4000 \\
\hline NEC SX-4/1A(1 proc.8.0ns) & R7.1 -fopp $\mathrm{f}=\mathrm{x}$ inline & 467 & 1929 & 2000 \\
\hline NEC SX-4B/2A (2 proc. 8.8 ns) & & & 3204 & 3636 \\
\hline HP V2600 (16 proc $550 \mathrm{MHz})$ & & & 9068 & 35200 \\
\hline HP V2600 (8 proc $550 \mathrm{MHz})$ & & & 6323 & 17600 \\
\hline HP V2600 (4 proc $550 \mathrm{MHz})$ & & & 3448 & 8800 \\
\hline HP V2600 (2 proc $550 \mathrm{MHz})$ & & & 2030 & 4400 \\
\hline Hewlett-Packard V2600(550 MHz) & $\mathrm{f} 77+\mathrm{O} 3+$ Oinline $=$ daxpy & 465 & 1221 & 2200 \\
\hline Compaq 8400 6/575(8proc 1.7 ns) & & & 5305 & 9600 \\
\hline Compaq 8400 6/575(6proc $1.7 \mathrm{~ns})$ & & & 4085 & 6900 \\
\hline Compaq 8400 6/575(4proc 1.7 ns) & & & 3003 & 4600 \\
\hline Compaq 8400 6/575(2proc 1.7 ns) & & & 1615 & 2300 \\
\hline Compaq 8400 6/575(1proc $1.7 \mathrm{~ns})$ & $\begin{array}{l}\text { kf77 -fkapargs }=\text { '-inline }=\text { daxpy }- \text { ur }=12 \\
\text {-tune ev6 -O5 }\end{array}$ & 460 & 847 & 1150 \\
\hline NEC SX-4B/e (1 proc. $8.8 \mathrm{~ns})$ & R7.1 -fopp $\mathrm{f}=\mathrm{x}$ inline & 454 & 890 & 909 \\
\hline AMD Opteron (1 proc. $1200 \mathrm{MHz})$ & $\begin{array}{l}\text { g77 -O3 -fforce-addr } \\
\text {-fomit-frame-pointer -funroll-loops } \\
\text {-frerun-cse-after-loop -frerun-loop-opt } \\
\text {-falign-functions=4 -static -s } \\
\text {-fexpensive-optimizations } \\
\text {-fschedule-insns2 }\end{array}$ & 443 & & 2400 \\
\hline Compaq Alpha Server DS20/500MHz & kf77 - fkapargs $='-$ inline $=$ daxpy - ur $=12$ & 440 & & 1000 \\
\hline
\end{tabular}




\begin{tabular}{|c|c|c|c|c|}
\hline Computer & $\begin{array}{c}\text { "LINPACK Benchmark" } \\
\text { OS/Compiler }\end{array}$ & $\begin{array}{c}\mathbf{n}=\mathbf{1 0 0} \\
\text { Mflop/s }\end{array}$ & $\begin{array}{c}\text { “TPP" } \\
\text { Best } \\
\text { Effort } \\
\text { n=1000 } \\
\text { Mflop/s }\end{array}$ & $\begin{array}{l}\text { "Theoritical } \\
\text { Peak" } \\
\text { Mflop/s }\end{array}$ \\
\hline & -tune ev6 -O5 & & & \\
\hline Compaq 8200 6/575(6proc 1.7 ns) & & & 3981 & 6900 \\
\hline Compaq 8200 6/575(4proc 1.7 ns) & & & 3003 & 4600 \\
\hline Compaq 8200 6/575(2proc 1.7 ns) & & & 1615 & 2300 \\
\hline Compaq 8200 6/575(1proc 1.7 ns) & $\begin{array}{l}\text { kf77 -fkapargs='-inline }=\text { daxpy }- \text { ur }=12 \\
\text {-tune ev6 -O5 }\end{array}$ & 431 & 831 & 1150 \\
\hline NEC SX-4B/1A (1 proc. $8.8 \mathrm{~ns})$ & R7.1 -fopp $\mathrm{f}=\mathrm{x}$ inline & 427 & 1753 & 1818 \\
\hline IBM RS/6K 44P-270(4 proc $375 \mathrm{MHz})$ & & & 3879 & 6000 \\
\hline IBM RS/6K 44P-270(2 proc $375 \mathrm{MHz})$ & & & 2101 & 3000 \\
\hline IBM RS/6K 44P-270(1 proc $375 \mathrm{MHz})$ & $\begin{array}{l}\text {-O -Q -qfloat=hsflt -qarch=pwr3 } \\
\text {-qtune=pwr3 -Pv -Wp,-ea478,-g1 }\end{array}$ & 426 & 1109 & 1500 \\
\hline IBM RS/6K 7026-B08(4 proc $375 \mathrm{MHz})$ & & & 3879 & 6000 \\
\hline IBM RS/6K 7026-B08(2 proc $375 \mathrm{MHz})$ & & & 2101 & 3000 \\
\hline IBM RS/6K 7026-B08(1 proc $375 \mathrm{MHz})$ & $\begin{array}{l}\text {-O -Q -qfloat=hsflt -qarch=pwr3 } \\
\text {-qtune=pwr3 -Pv -Wp,-ea478,-g1 }\end{array}$ & 426 & 1109 & 1500 \\
\hline $\begin{array}{l}\text { IBM eServer pSeries } 640 \text { ( } 4 \text { proc, } 375 \mathrm{MHz}, 4 \mathrm{MB} \\
\text { L2) }\end{array}$ & & & 3879 & 6000 \\
\hline $\begin{array}{l}\text { IBM eServer pSeries } 640 \text { ( } 2 \text { proc, } 375 \mathrm{MHz}, 4 \mathrm{MB} \\
\text { L2) }\end{array}$ & & & 2101 & 3000 \\
\hline $\begin{array}{l}\text { IBM eServer pSeries } 640 \text { ( } 1 \text { proc, } 375 \mathrm{MHz}, 4 \mathrm{MB} \\
\text { L2) }\end{array}$ & $\begin{array}{l}\text {-qarch=pwr3 -qtune=pwr3 -Pv } \\
\text {-Wp,-ea478,-g1 }\end{array}$ & 426 & 1109 & 1500 \\
\hline IBM RS/6K 44P-270 (4 proc,375MHz,4MB L2) & & & 3879 & 6000 \\
\hline IBM RS/6K 44P-270 (2 proc,375MHz,4MB L2) & & & 2101 & 3000 \\
\hline IBM RS/6K 44P-270 (1 proc,375MHz,4MB L2) & $\begin{array}{l}-O-Q-q f l o a t=h s f l t-q a r c h=p w r 3 \\
\text {-qtune=pwr3 -Pv -Wp,-ea478,-g1 }\end{array}$ & 426 & 1109 & 1500 \\
\hline $\begin{array}{l}\text { IBM eServer pSeries } 640 \text { ( } 4 \text { proc, } 375 \mathrm{MHz}, 8 \mathrm{MB} \\
\text { L2) }\end{array}$ & & & 3902 & 6000 \\
\hline $\begin{array}{l}\text { IBM eServer pSeries } 640(2 \text { proc, } 375 \mathrm{MHz}, 8 \mathrm{MB} \\
\text { L2) }\end{array}$ & & & 2180 & 3000 \\
\hline $\begin{array}{l}\text { IBM eServer pSeries } 640 \text { ( } 1 \text { proc, } 375 \mathrm{MHz}, 8 \mathrm{MB} \\
\text { L2) }\end{array}$ & $\begin{array}{l}\text {-qarch=pwr3 -qtune=pwr3 -Pv } \\
-\mathrm{Wp},- \text { ea478,-g1 }\end{array}$ & 426 & 1234 & 1500 \\
\hline IBM RS/6K 44P-270 (4 proc,375MHz,8MB L2) & & & 3902 & 6000 \\
\hline IBM RS/6K 44P-270 (2 proc,375MHz,8MB L2) & & & 2180 & 3000 \\
\hline IBM RS/6K 44P-270 (1 proc,375MHz,8MB L2) & $\begin{array}{l}-\mathrm{O}-\mathrm{Q}-\mathrm{qfloat}=\mathrm{hsflt}-\mathrm{qarch}=\mathrm{pwr} 3 \\
\text {-qtune }=\text { pwr3 }-\mathrm{Pv}-\mathrm{Wp},-\mathrm{ea} 478,-\mathrm{g} 1\end{array}$ & 426 & 1234 & 1500 \\
\hline IBM RS/6K SP Power3(16 proc $375 \mathrm{MHz})$ & & & 7699 & 24000 \\
\hline IBM RS/6K SP Power3(12 proc $375 \mathrm{MHz})$ & & & 7187 & 18000 \\
\hline IBM RS/6K SP Power3(8 proc $375 \mathrm{MHz})$ & & & 5928 & 12000 \\
\hline IBM RS/6K SP Power3(4 proc $375 \mathrm{MHz}$ ) & & & 3728 & 6000 \\
\hline IBM RS/6K SP Power3(1 proc $375 \mathrm{MHz}$ ) & $\begin{array}{l}\text {-O -Q -qfloat=hsflt }- \text { qarch=pwr3 } \\
\text {-qtune }=\text { pwr3 }-P v-W p,-e a 478,- \text { g1 }\end{array}$ & 424 & 1208 & 1500 \\
\hline Cray $3-128$ (4 proc. $2.11 \mathrm{~ns})$ & CSOS 1.0 level 129 & 421 & 2862 & 3792 \\
\hline
\end{tabular}




\begin{tabular}{|c|c|c|c|c|}
\hline Computer & $\begin{array}{c}\text { "LINPACK Benchmark" } \\
\text { OS/Compiler }\end{array}$ & $\begin{array}{c}n=100 \\
\text { Mflop/s }\end{array}$ & $\begin{array}{c}\text { “TPP" } \\
\text { Best } \\
\text { Effort } \\
\text { n=1000 } \\
\text { Mflop/s }\end{array}$ & $\begin{array}{l}\text { "Theoritical } \\
\text { Peak" } \\
\text { Mflop/s }\end{array}$ \\
\hline Compaq/DEC Alpha 21264 EV67 500 MHz & -O5 -arch host -tune host & 412 & 637 & 1000 \\
\hline Hitachi S-3800/480(4 proc 2 ns) & & & 20640 & 32000 \\
\hline Hitachi S-3800/380(3 proc 2 ns) & & & 16880 & 24000 \\
\hline Hitachi S-3800/280(2 proc 2 ns) & & & 12190 & 16000 \\
\hline Hitachi S-3800/180(1 proc 2 ns) & OSF/1 MJ FORTRAN:V03-00 & 408 & 6431 & 8000 \\
\hline IBM RS/6K SP (4 proc $375 \mathrm{MHz}$ ) & & & 3700 & 6000 \\
\hline IBM RS/6K SP (2 proc $375 \mathrm{MHz})$ & & & 2166 & 3000 \\
\hline IBM RS/6K SP (1 proc $375 \mathrm{MHz})$ & $\begin{array}{l}\text { xlf 6.1.0.3 -O3 -Q -qfloat=hsflt } \\
\text {-qarch=pwr3 -qtune=pwr3 -Pv } \\
\text {-Wp,-ea478,-g1 }\end{array}$ & 409 & 1236 & 1500 \\
\hline Cray 3-128 (2 proc. $2.11 \mathrm{~ns})$ & CSOS 1.0 level 129 & 393 & 1622 & 1896 \\
\hline Cray C90 (4 proc. $4.2 \mathrm{~ns})$ & CF77 5.0 -Zp -Wd-e68 & 388 & 3275 & 3810 \\
\hline Cray C90 (2 proc. $4.2 \mathrm{~ns})$ & CF77 5.0 -Zp -Wd-e68 & 387 & 1703 & 1905 \\
\hline Cray C90 (1 proc. $4.2 \mathrm{~ns})$ & CF77 $5.0-Z p$-Wd-e68 & 387 & 902 & 952 \\
\hline HP N4000 (8 proc. $440 \mathrm{MHz})$ & & & 6410 & 14080 \\
\hline HP N4000 (4 proc. $440 \mathrm{MHz}$ ) & & & 3724 & 7040 \\
\hline HP N4000 (2 proc. $440 \mathrm{MHz})$ & & & 2212 & 3520 \\
\hline HP N4000 (1 proc. $440 \mathrm{MHz})$ & $\mathrm{f} 77+\mathrm{O} 3+$ Oinline $=$ daxpy & 375 & 1290 & 1760 \\
\hline HP V2500 (16 proc. $440 \mathrm{MHz})$ & & & 8217 & 28160 \\
\hline HP V2500 (12 proc. $440 \mathrm{MHz})$ & & & 6914 & 21120 \\
\hline HP V2500 (8 proc. $440 \mathrm{MHz}$ ) & & & 5111 & 14080 \\
\hline HP V2500 (4 proc. $440 \mathrm{MHz})$ & & & 3041 & 7040 \\
\hline HP V2500 (2 proc. $440 \mathrm{MHz})$ & & & 1751 & 3520 \\
\hline HP V2500 (1 proc. $440 \mathrm{MHz})$ & $\mathrm{f} 77+\mathrm{O} 3+$ Oinline $=$ daxpy & 375 & 1047 & 1760 \\
\hline NEC SX-3/44R (4 proc. 2.5 ns) & & & 15120 & 25600 \\
\hline NEC SX-3/42R (4 proc. $2.5 \mathrm{~ns})$ & & & 8950 & 12800 \\
\hline NEC SX-3/41R (4 proc. 2.5 ns) & & & 4815 & 6400 \\
\hline NEC SX-3/34R (3 proc. $2.5 \mathrm{~ns})$ & & & 12730 & 19200 \\
\hline NEC SX-3/32R (3 proc. $2.5 \mathrm{~ns})$ & & & 6718 & 9600 \\
\hline NEC SX-3/31R (3 proc. $2.5 \mathrm{~ns}$ ) & & & 3638 & 4800 \\
\hline NEC SX-3/24R (2 proc. 2.5 ns) & & & 9454 & 12800 \\
\hline NEC SX-3/22R (2 proc. 2.5 ns) & & & 5116 & 6400 \\
\hline NEC SX-3/21R (2 proc. 2.5 ns) & & & 2627 & 3200 \\
\hline NEC SX-3/14R (1 proc. 2.5 ns) & f77sx 040 R2.2 -pi*:* & 368 & 5199 & 6400 \\
\hline NEC SX-3/12R (1 proc. $2.5 \mathrm{~ns})$ & f77sx 040 R2.2 -pi*:* & 368 & 2757 & 3200 \\
\hline Intel P4 $1700 \mathrm{MHz}$ & $\begin{array}{l}\text { g77 -O3 -fomit-frame-pointer } \\
\text {-funroll-loops }\end{array}$ & 363 & 1393 & 3400 \\
\hline
\end{tabular}




\begin{tabular}{|c|c|c|c|c|}
\hline Computer & $\begin{array}{c}\text { "LINPACK Benchmark" } \\
\text { OS/Compiler }\end{array}$ & $\begin{array}{c}n=100 \\
\text { Mflop/s }\end{array}$ & $\begin{array}{c}\text { "TPP" } \\
\text { Best } \\
\text { Effort } \\
\text { n=1000 } \\
\text { Mflop/s }\end{array}$ & $\begin{array}{l}\text { "Theoritical } \\
\text { Peak" } \\
\text { Mflop/s }\end{array}$ \\
\hline $\begin{array}{l}\text { IBM eServer pSeries 620/6F1 } 6 \text { CPUs(668 MHz } 8 \\
\text { MB L2) }\end{array}$ & & & 4529 & 8016 \\
\hline $\begin{array}{l}\text { IBM eServer pSeries 620/6F } 14 \text { CPUs( } 600 \text { MHz } 4 \\
\text { MB L2) }\end{array}$ & & & 3144 & 4800 \\
\hline $\begin{array}{l}\text { IBM eServer pSeries 620/6F } 12 \text { CPUs( } 600 \mathrm{MHz} 4 \\
\text { MB L2) }\end{array}$ & & & 1650 & 2400 \\
\hline $\begin{array}{l}\text { IBM eServer pSeries 620/6F1 } 1 \text { CPU (600 MHz } 2 \\
\text { MB L2) }\end{array}$ & $\begin{array}{l}\text { xlf } 7.1-O-Q-q f l o a t=h s f l t-q a r c h=p w r 3 \\
\text {-qtune=pwr3 -Pv -Wp,-ea478,-g1 }\end{array}$ & 360 & 833 & 1200 \\
\hline $\begin{array}{l}\text { IBM eServer pSeries 660/6H1 } 6 \text { CPUs( } 668 \text { MHz } 8 \\
\text { MB L2) }\end{array}$ & & & 4529 & 8016 \\
\hline $\begin{array}{l}\text { IBM eServer pSeries 660/6H1 } 4 \text { CPUs( } 600 \mathrm{MHz} 4 \\
\text { MB L2) }\end{array}$ & & & 3144 & 4800 \\
\hline $\begin{array}{l}\text { IBM eServer pSeries 660/6H1 } 2 \text { CPUs( } 600 \text { MHz } 4 \\
\text { MB L2) }\end{array}$ & & & 1650 & 2400 \\
\hline $\begin{array}{l}\text { IBM eServer pSeries 660/6H1 } 1 \text { CPU (600 MHz } 2 \\
\text { MB L2) }\end{array}$ & $\begin{array}{l}\text { xlf } 7.1-O-Q-q f l o a t=h s f l t-q a r c h=p w r 3 \\
\text {-qtune=pwr3 -Pv -Wp,-ea478,-g1 }\end{array}$ & 360 & 833 & 1200 \\
\hline $\begin{array}{l}\text { Acer TravelMate 803LMi Intel Pentium M } \\
\text { (1.6GHz) }\end{array}$ & $\mathrm{f} 77-\mathrm{O} 3$ & 352 & & 3200 \\
\hline Sun UltraSparc III $750 \mathrm{MHz}$ & $\begin{array}{l}\text {-fast }- \text { native }-\mathrm{xsafe}=\text { mem } \text {-dalign }-\mathrm{xO} 5 \\
\text {-xarch }=\text { v8plusa }- \text { xchip }=\text { ultra }\end{array}$ & 343 & 769 & 1500 \\
\hline Cray 3-128 (1 proc. $2.11 \mathrm{~ns})$ & CSOS 1.0 level 129 & 327 & 876 & 948 \\
\hline Intel P4 $1500 \mathrm{MHz}$ & $\begin{array}{l}\text { g77 -O3 -fomit-frame-pointer } \\
\text {-funroll-loops }\end{array}$ & 326 & 1311 & 3000 \\
\hline Gigabyte GA-7VX/AMD Athlon(700 MHz) & ifc -tpp6 -O3 & 317 & 772 & 1400 \\
\hline IBM RS6000/397(160 MHz ThinNode) & $\begin{array}{l}\text {-qarch=pwr2 -qhot -O3 -Pv } \\
\text {-Wp,-ea478,-g1 }\end{array}$ & 315 & 532 & 640 \\
\hline Compaq XP1000 (500 MHz) & $\begin{array}{l}\text { kf77 -tune ev6 -O5 } \\
\text {-fkapargs='-inline=daxpy -ur=12, }\end{array}$ & 335 & & 1000 \\
\hline NEC SX-3/44 (4 proc. 2.9 ns) & & & 13420 & 22000 \\
\hline NEC SX-3/24 (2 proc. 2.9 ns) & & & 8149 & 11000 \\
\hline NEC SX-3/42 (4 proc. $2.9 \mathrm{~ns})$ & & & 7752 & 11000 \\
\hline NEC SX-3/22 (2 proc. 2.9 ns) & & & 4404 & 5500 \\
\hline NEC SX-3/14 (1 proc. $2.9 \mathrm{~ns})$ & f77sx 020 R1.13 -pi*:* & 314 & 4511 & 5500 \\
\hline NEC SX-3/12 (1 proc. 2.9 ns) & f77sx 020 R1.13 -pi*:* & 313 & 2283 & 2750 \\
\hline DEC 8400 5/625(8 proc,612 MHz) & & & 3608 & 9792 \\
\hline DEC 8400 5/625(4 proc,612 MHz) & & & 2377 & 4896 \\
\hline DEC 8400 5/625(2 proc, $612 \mathrm{MHz})$ & & & 1375 & 2448 \\
\hline DEC 8400 5/625(1 proc,612 MHz) & f77 -O5 -fast & 287 & 764 & 1224 \\
\hline Apple PowerPC G4 $1 \mathrm{GHz}$ & $\begin{array}{l}f 90-\mathrm{q}-\mathrm{YEXT} S \mathrm{SFX}=-\mathrm{O} 3 \\
-\mathrm{YEXT} N \mathrm{NAMES}=\mathrm{LCS}-\mathrm{YCFRL}=1\end{array}$ & 284 & 1000 & 2000 \\
\hline Cray Y-MP/832 (8 proc. 6 ns) & CF77 4.0 -Zp -Wd-e68 & 275 & 2144 & 2667 \\
\hline Compaq Alpha Server ds20/500MHz & -fast -O5 -arch ev6 -tune ev6 & 270 & & 1000 \\
\hline DEC 8200 5/625(8 proc,612 MHz) & & & 2696 & 9792 \\
\hline DEC 8200 5/625(4 proc, $612 \mathrm{MHz})$ & & & 2313 & 4896 \\
\hline
\end{tabular}




\begin{tabular}{|c|c|c|c|c|}
\hline Computer & $\begin{array}{c}\text { "LINPACK Benchmark" } \\
\text { OS/Compiler }\end{array}$ & $\begin{array}{c}n=100 \\
\text { Mflop/s }\end{array}$ & $\begin{array}{c}\text { “TPP" } \\
\text { Best } \\
\text { Effort } \\
\text { n=1000 } \\
\text { Mflop/s }\end{array}$ & $\begin{array}{l}\text { "Theoritical } \\
\text { Peak" } \\
\text { Mflop/s }\end{array}$ \\
\hline DEC $82005 / 625(2$ proc, $612 \mathrm{MHz})$ & & & 1366 & 2448 \\
\hline DEC 8200 5/625(1 proc,612 MHz) & f77 -O5 -fast & 268 & 750 & 1224 \\
\hline IBM RS6K/595(135 MHz WideNode) & $\begin{array}{l}\text {-qarch=pwr2 -qhot }-\mathrm{O} 3-\mathrm{Pv} \\
\text {-Wp,-ea478,-g1 }\end{array}$ & 265 & 440 & 540 \\
\hline IBM RS6K SP Power3SMP(8 Proc 222 MHz) & & & 3516 & 7104 \\
\hline IBM RS6K SP Power3SMP(6 Proc $222 \mathrm{MHz}$ ) & & & 3014 & 5328 \\
\hline IBM RS6K SP Power3SMP(4 Proc 222 MHz) & & & 2153 & 3552 \\
\hline IBM RS6K SP Power3SMP(2 Proc 222 MHz) & & & 1247 & 1776 \\
\hline AMD Athlon (600 Mhz) & $\begin{array}{l}\text { g77 -O3 -s -funroll-loops } \\
\text {-fomit-frame-pointer }\end{array}$ & 260 & 557 & 1200 \\
\hline IBM RS6K SP Power3SMP(1 Proc 222 MHz) & $\begin{array}{l}-\mathrm{O} 3-\mathrm{Q} \text {-qfloat=hsflt }-\mathrm{qarch}=\mathrm{pwr} 3 \\
-\mathrm{qtune}=\text { pwr3 -bnso -bI:/lib/syscalls.exp } \\
-\mathrm{Pv}\end{array}$ & 250 & 684 & 888 \\
\hline Fujitsu VP2600/10 (3.2 ns) & FORTRAN77 EX/VP V11L10 & 249 & 4009 & 5000 \\
\hline DEC 500/500 (1 proc, $500 \mathrm{MHz})$ & $\begin{array}{l}\text { kf77 -inline }=\text { daxpy }- \text { ur }=3 \text {-fast }-\mathrm{O} 5 \text {-tune } \\
\text { ev5 }\end{array}$ & 235 & 590 & 1000 \\
\hline Intel Pentium III $933 \mathrm{MHz}$ & $\begin{array}{l}\text { g77 -O3 -fomit-frame-pointer } \\
\text {-funroll-loops }\end{array}$ & 234 & 514 & 933 \\
\hline IBM P2SC (120 MHz Thin Node) & $\begin{array}{l}\text {-qarch=pwr2 -qhot -O3 -Pv } \\
\text {-Wp,-ea478,-g1 -funroll-loops }\end{array}$ & 233 & 406 & 480 \\
\hline Apple PowerPC G4 533 MHz & $\begin{array}{l}\text { g77 -O3 -fomit-frame-pointer } \\
\text {-funroll-loops }\end{array}$ & 231 & 478 & 1066 \\
\hline DEC PersonalWorkstation 600 & $\begin{array}{l}\text {-O5 -fast -tune ev56 -inline all -speculate } \\
\text { all }\end{array}$ & 227 & & 1200 \\
\hline Cray Y-MP/832 (4 proc. $6 \mathrm{~ns}$ ) & CF77 4.0 -Zp -Wd-e68 & 226 & 1159 & 1333 \\
\hline Sun Ultra $80(4$ proc $450 \mathrm{MHz})$ & & & 2062.0 & 3600 \\
\hline Sun Ultra $80(3$ proc $450 \mathrm{MHz})$ & & & 1615.0 & 2700 \\
\hline Sun Ultra $80(2$ proc $450 \mathrm{MHz})$ & & & 1172.0 & 1800 \\
\hline Sun Ultra 80 (450MHz/4MB L2) & -fast $-\mathrm{xO} 5-\mathrm{xarch}=\mathrm{v} 8$ plusa $-\mathrm{xchip}=$ ultra & 208 & 607 & 900 \\
\hline Fujitsu VPP500/1(1 proc. 10 ns $)$ & FORTRAN77EX/VP V12L20 & 206 & 1490 & 1600 \\
\hline DEC 8400 5/440(8 proc, $440 \mathrm{MHz})$ & $\begin{array}{l}\text { kf77 -inline }=\text { daxpy }- \text { ur }=3 \text {-fast }-05 \text {-tune } \\
\text { ev5 }\end{array}$ & & 3112 & 7040 \\
\hline DEC 8100 5/440(4 proc, $440 \mathrm{MHz})$ & $\begin{array}{l}\text { kf77 -inline }=\text { daxpy }- \text { ur }=3 \text {-fast }-05 \text {-tune } \\
\text { ev5 }\end{array}$ & & 1945 & 3520 \\
\hline DEC 8100 5/440(2 proc, $440 \mathrm{MHz})$ & $\begin{array}{l}\text { kf77 -inline }=\text { daxpy }- \text { ur }=3 \text {-fast }-\mathrm{O} 5 \text {-tune } \\
\text { ev5 }\end{array}$ & & 1090 & 1760 \\
\hline DEC 8100 5/440(1 proc, $440 \mathrm{MHz})$ & $\begin{array}{l}\text { kf77 -inline }=\text { daxpy }- \text { ur }=3 \text {-fast }-\mathrm{O} 5 \text {-tune } \\
\text { ev5 }\end{array}$ & 205 & 588 & 880 \\
\hline Cray Y-MP M98 (8 proc. 6 ns) & CF77 5.0 -Zp -Wd-e68 & 204 & 1733 & 2666 \\
\hline Fujitsu VX/1 (1 proc. $7 \mathrm{~ns})$ & Fortran90/VP V10L10 & 203 & 1936 & 2200 \\
\hline Fujitsu VPP300/1 (1 proc. 7 ns) & Fortran90/VP V10L10 & 203 & 1936 & 2200 \\
\hline Fujitsu VPP700/1 (1 proc. 7 ns) & Fortran90/VP V10L10 & 203 & 1936 & 2200 \\
\hline Fujitsu VP2200/10 (3.2 ns) & FORTRAN77 EX/VP V12L10 & 203 & 1048 & 1250 \\
\hline HP Exemplar V-Class(16 proc. $240 \mathrm{MHz}$ ) & $+\mathrm{O} 3+$ Oinline $=$ daxpy & & 5935 & 15360 \\
\hline
\end{tabular}




\begin{tabular}{|c|c|c|c|c|}
\hline Computer & $\begin{array}{c}\text { "LINPACK Benchmark" } \\
\text { OS/Compiler }\end{array}$ & $\begin{array}{c}n=100 \\
\text { Mflop/s }\end{array}$ & $\begin{array}{c}\text { "TPP" } \\
\text { Best } \\
\text { Effort } \\
\text { n=1000 } \\
\text { Mflop/s }\end{array}$ & \begin{tabular}{|c} 
"Theoritical \\
Peak" \\
Mflop/s
\end{tabular} \\
\hline HP Exemplar V-Class(14 proc. $240 \mathrm{MHz}$ ) & $+\mathrm{O} 3+$ Oinline $=$ daxpy & & 5394 & 13440 \\
\hline HP Exemplar V-Class(12 proc. $240 \mathrm{MHz}$ ) & $+\mathrm{O} 3+$ Oinline $=$ daxpy & & 5202 & 11520 \\
\hline HP Exemplar V-Class(10 proc. $240 \mathrm{MHz}$ ) & $+\mathrm{O} 3+$ Oinline $=$ daxpy & & 4585 & 9600 \\
\hline HP Exemplar V-Class(8 proc. $240 \mathrm{MHz}$ ) & $+\mathrm{O} 3+$ Oinline $=$ daxpy & & 4125 & 7680 \\
\hline HP Exemplar V-Class(6 proc. $240 \mathrm{MHz}$ ) & $+\mathrm{O} 3+$ Oinline $=$ daxpy & & 3350 & 4760 \\
\hline HP Exemplar V-Class(4 proc. $240 \mathrm{MHz}$ ) & $+\mathrm{O} 3+$ Oinline $=$ daxpy & & 2414 & 3840 \\
\hline HP Exemplar V-Class(2 proc. $240 \mathrm{MHz}$ ) & $+\mathrm{O} 3+$ Oinline $=$ daxpy & & 1260 & 1920 \\
\hline HP Exemplar V-Class(1 proc. $240 \mathrm{MHz}$ ) & HP-UX $11.0+\mathrm{O} 3+$ Oinline $=$ daxpy & 203 & 743 & 960 \\
\hline Cray $2 \mathrm{~S} / 4-128$ (4 proc. $4.1 \mathrm{~ns})$ & CSOS 1.0 level 129 & 202 & 1406 & 1951 \\
\hline NEC SX-3/11R (1 proc. $2.5 \mathrm{~ns})$ & f77sx 040 R2.2 -pi*:* & 202 & 1418 & 1600 \\
\hline NEC SX-3/1LR (1 proc. 2.5 ns) & f77sx 040 R2.2 -pi*:* & 201 & 767 & 800 \\
\hline Hewlett-Packard C240 $236 \mathrm{MHz}$ & $+\mathrm{O} 3+$ Oinline $=$ daxpy & 197 & 667 & 944 \\
\hline Intel Pentium III 933 MHz & g77-O3 & 192 & 507 & 933 \\
\hline DEC 500/400 (1 proc, $400 \mathrm{MHz})$ & $\begin{array}{l}\text { kf77 -inline }=\text { daxpy }- \text { ur }=3 \text {-fast -O5 -tune } \\
\text { ev5 }\end{array}$ & 189 & 449 & 800 \\
\hline DEC 4100 5/400(4 proc, $400 \mathrm{MHz})$ & $\begin{array}{l}\text { kf77 -inline }=\text { daxpy }- \text { ur }=3 \text {-fast -O5 -tune } \\
\text { ev5 }\end{array}$ & & 1821 & 3200 \\
\hline DEC 4100 5/400(2 proc, $400 \mathrm{MHz})$ & $\begin{array}{l}\text { kf77 -inline }=\text { daxpy }- \text { ur }=3 \text {-fast -O5 -tune } \\
\text { ev5 }\end{array}$ & & 1001 & 1600 \\
\hline DEC 4100 5/400(1 proc, $400 \mathrm{MHz})$ & $\begin{array}{l}\text { kf77 -inline }=\text { daxpy }- \text { ur }=3 \text {-fast -O5 -tune } \\
\text { ev5 }\end{array}$ & 189 & 531 & 800 \\
\hline DEC 1000A 5/400(1 proc, $400 \mathrm{MHz})$ & $\begin{array}{l}\text { kf77 -inline }=\text { daxpy }- \text { ur }=3 \text {-fast -O5 -tune } \\
\text { ev5 }\end{array}$ & 187 & 440 & 800 \\
\hline Sun HPC 450 (400 MHz, 4 proc) & & & 1841 & 3200 \\
\hline Sun HPC 450 (400 MHz, 2 proc) & & & 1050 & 1600 \\
\hline Sun HPC 450 (400 MHz, 4MB L2) & -fast $-\mathrm{xO} 5-\mathrm{xarch}=\mathrm{v} 8$ plusa $-\mathrm{xchip}=$ ultra & 183 & 552 & 800 \\
\hline Cray Y-MP/832 (2 proc. $6 \mathrm{~ns})$ & CF77 5.0 -Zp -Wd-e68 & 181 & 604 & 667 \\
\hline Cray X-MP/416 (4 proc. $8.5 \mathrm{~ns}$ ) & CF77 4.0 -Zp -Wd-e68 & 178 & 822 & 940 \\
\hline Cray Y-MP M98 (4 proc. $6 \mathrm{~ns}$ ) & CF77 5.0-Zp -Wd-e68 & 177 & 1114 & 1333 \\
\hline Sun UltraSparc II $300 \mathrm{MHz}$ & $\begin{array}{l}\text {-fast -native -xsafe }=\text { mem -dalign }-\mathrm{xO} 5 \\
-\mathrm{xarch}=\mathrm{v} 8 \text { plusa }-\mathrm{xchip}=\text { ultra }\end{array}$ & 176 & 296 & 600 \\
\hline SGI Origin 2000 ( $300 \mathrm{Mhz}, 16$ proc) & & & 3970 & 9600 \\
\hline SGI Origin 2000 (300 Mhz, 8 proc) & & & 3032 & 4800 \\
\hline SGI Origin 2000 (300 Mhz, 4 proc) & & & 1957 & 2400 \\
\hline SGI Origin 2000 (300 Mhz, 2 proc) & & & 1074 & 1200 \\
\hline SGI Origin $2000(300 \mathrm{Mhz})$ & $\begin{array}{l}\text { f77 -IPA -O3 -n32 -mips4 -r10000 } \\
\text {-call_shared -TENV:X=4 } \\
\text {-OPT:IEEE_arithmetic=3:roundoff=3 } \\
\text {-LNO:blocking=off:ou_max=6:pf2=0 } \\
\text {-INLINE:array_bounds }\end{array}$ & 173 & 553 & 600 \\
\hline NEC SX-3/11 (1 proc. $2.9 \mathrm{~ns})$ & f77sx 020 R1.13 -pi*:* & 173 & 1223 & 1370 \\
\hline Sun UltraSparc II $300 \mathrm{MHz}$ & -fast -native $-\mathrm{xsafe}=$ mem -dalign $-\mathrm{xO} 5$ & 172 & 285 & 600 \\
\hline
\end{tabular}




\begin{tabular}{|c|c|c|c|c|}
\hline Computer & $\begin{array}{c}\text { "LINPACK Benchmark" } \\
\text { OS/Compiler }\end{array}$ & $\begin{array}{c}n=100 \\
\text { Mflop/s }\end{array}$ & $\begin{array}{c}\text { "TPP" } \\
\text { Best } \\
\text { Effort } \\
\text { n=1000 } \\
\text { Mflop/s }\end{array}$ & $\begin{array}{c}\text { "Theoritical } \\
\text { Peak" } \\
\text { Mflop/s }\end{array}$ \\
\hline & -xarch $=v 8$ plusa $-\mathrm{xchip}=$ ultra & & & \\
\hline NEC SX-3/1L (1 proc. $2.9 \mathrm{~ns})$ & f77sx 020 R1.13 -pi*:* & 171 & 661 & 680 \\
\hline SGI Octane (360 MHz) IP30 & $\mathrm{f} 77-\mathrm{O}$ & 170 & & 720 \\
\hline Fujitsu VP2400/10 (4 ns) & FORTRAN77 EX/VP V11L10 & 170 & 1688 & 2000 \\
\hline HP Exemplar V-Class(16 proc.200 MHz) & HP-UX 11.0 & & 4832 & 12800 \\
\hline HP Exemplar V-Class(14 proc.200 MHz) & HP-UX 11.0 & & 4442 & 11200 \\
\hline HP Exemplar V-Class(12 proc. $200 \mathrm{MHz}$ ) & HP-UX 11.0 & & 4109 & 8400 \\
\hline HP Exemplar V-Class(10 proc. $200 \mathrm{MHz}$ ) & HP-UX 11.0 & & 3506 & 8000 \\
\hline HP Exemplar V-Class(8 proc. $200 \mathrm{MHz}$ ) & HP-UX 11.0 & & 3206 & 6400 \\
\hline HP Exemplar V-Class(6 proc.200 MHz) & HP-UX 11.0 & & 2608 & 4200 \\
\hline HP Exemplar V-Class(4 proc. $200 \mathrm{MHz}$ ) & HP-UX 11.0 & & 1912 & 3200 \\
\hline HP Exemplar V-Class(2 proc. $200 \mathrm{MHz}$ ) & HP-UX 11.0 & & 1082 & 1600 \\
\hline HP Exemplar V-Class(1 proc. $200 \mathrm{MHz}$ ) & HP-UX $11.0+\mathrm{O} 3+$ Oinline=daxpy & 169 & 613 & 800 \\
\hline SGI Octane R12000 IP30 $270 \mathrm{MHz}$ & $\begin{array}{l}\text {-O3 -64 -OPT:Olimit }=15000 \\
\text {-TARG:platform=IP30 } \\
\text {-LNO:blocking=OFF }\end{array}$ & 169 & 400 & 540 \\
\hline Compaq Alpha 21164 EV56 533 MHz & $\begin{array}{l}\text { g77 -O3 -fomit-frame-pointer } \\
\text {-funroll-loops }\end{array}$ & 168 & 508 & 1066 \\
\hline Cray $2 \mathrm{~S} / 4-128$ (2 proc. $4.1 \mathrm{~ns})$ & CSOS 1.0 level 129 & 167 & 741 & 976 \\
\hline Hewlett-Packard C200 $200 \mathrm{MHz}$ & $+\mathrm{O} 3+$ Oinline $=$ daxpy & 166 & 550 & 800 \\
\hline DEC 8400 5/350 (1 proc $350 \mathrm{MHz})$ & $\begin{array}{l}\text { kf77 -fkapargs='-inline=daxpy } \\
\text {-ur3=100' -tune ev5 -O5 -assume } \\
\text { nounderscore }\end{array}$ & 164 & 510 & 700 \\
\hline DEC 8400 5/300 (8 proc $300 \mathrm{MHz})$ & & & 2282 & 4800 \\
\hline DEC 8400 5/300 (6 proc $300 \mathrm{MHz})$ & & & 1902 & 3600 \\
\hline DEC 8400 5/300 (4 proc $300 \mathrm{MHz}$ ) & & & 1351 & 2400 \\
\hline DEC 8400 5/300 (2 proc $300 \mathrm{MHz})$ & & & 757 & 1200 \\
\hline Cray Y-MP/832 (1 proc. 6 ns) & CF77 5.0 -Zp -Wd-e68 & 161 & 324 & 333 \\
\hline ASUS P2B-F/Celeron(433 MHz,1 Proc) & ifc -tpp6-O3 & 160 & 263 & 433 \\
\hline Convex C4/XA-4(4 proc) (7.41 ns) & fc9.0.0.5 -tm c4 -O3 -ds -ep 4 -is . & 160 & 2531 & 3240 \\
\hline Hewlett-Packard K460-EG 180 MHz & + Oall + Oinline $=$ daxpy & 158 & 510 & 720 \\
\hline Hewlett-Packard C180-XP $180 \mathrm{MHz}$ & + Oall + Oinline $=$ daxpy & 158 & 480 & 720 \\
\hline HP Exemplar S-Class (16 proc) & SPP-UX 5.2 & & 4609 & 11520 \\
\hline HP Exemplar S-Class (14 proc) & SPP-UX 5.2 & & 4217 & 10080 \\
\hline HP Exemplar S-Class (12 proc) & SPP-UX 5.2 & & 4019 & 8640 \\
\hline HP Exemplar S-Class (10 proc) & SPP-UX 5.2 & & 3389 & 7200 \\
\hline HP Exemplar S-Class (8 proc) & SPP-UX 5.2 & & 2979 & 5760 \\
\hline HP Exemplar S-Class (6 proc) & SPP-UX 5.2 & & 2305 & 4320 \\
\hline HP Exemplar S-Class (4 proc) & SPP-UX 5.2 & & 1629 & 2880 \\
\hline
\end{tabular}




\begin{tabular}{|c|c|c|c|c|}
\hline Computer & $\begin{array}{c}\text { "LINPACK Benchmark" } \\
\text { OS/Compiler }\end{array}$ & $\begin{array}{c}n=100 \\
\text { Mflop/s }\end{array}$ & $\begin{array}{c}\text { "TPP" } \\
\text { Best } \\
\text { Effort } \\
\text { n=1000 } \\
\text { Mflop/s }\end{array}$ & $\begin{array}{l}\text { "Theoritical } \\
\text { Peak" } \\
\text { Mflop/s }\end{array}$ \\
\hline HP Exemplar S-Class (2 proc) & SPP-UX 5.2 & & 967 & 1440 \\
\hline HP Exemplar S-Class(1 proc) & SPP-UX 5.2+Oall +Oinline=daxpy & 156 & 545 & 720 \\
\hline Sun UltraSPARC II(30 proc)336MHz & & & 5187 & 20160 \\
\hline Sun UltraSPARC II(24 proc)336MHz & & & 4755 & 16128 \\
\hline Sun UltraSPARC II(16 proc)336MHz & & & 3981 & 10752 \\
\hline Sun UltraSPARC II(14 proc)336MHz & & & 3721 & 9408 \\
\hline Sun UltraSPARC II(8 proc)336MHz & & & 2481 & 5376 \\
\hline Sun UltraSPARC II(6 proc)336MHz & & & 1990 & 4032 \\
\hline Sun UltraSPARC II(4 proc)336MHz & & & 1438 & 2688 \\
\hline Sun UltraSPARC II(2 proc)336MHz & & & 843 & 1344 \\
\hline Sun UltraSPARC II(1 proc)336MHz & $\begin{array}{l}\text {-fast }-\mathrm{xO} 5-\mathrm{xarch}=\mathrm{v} 8 \text { plusa }-\mathrm{xchip}=\text { ultra } \\
-\mathrm{o}\end{array}$ & 154 & 461 & 672 \\
\hline Cray Y-MP M98 ( 2 proc. 6 ns) & CF77 5.0 -Zp -Wd-e68 & 154 & 596 & 666 \\
\hline DEC AlphaStation 600 5/333 MHz & $\begin{array}{l}\text {-fkapargs }={ }^{\prime} \text {-inline }=\text { daxpy }- \text { ur } 3=100 \\
\text {-tune ev5 -O5 }\end{array}$ & 153 & & 666 \\
\hline Convex C4/XA-3(3 proc) (7.41 ns) & fc9.0.0.5 -tm c4 -O3 -ds -ep 3 -is . & 151 & 1933 & 2430 \\
\hline Cray Y-MP M98 (1 proc. 6 ns) & CF77 5.0 -Zp -Wd-e68 & 150 & 307 & 333 \\
\hline Cray Y-MP M92 (2 proc. 6 ns) & CF77 5.0 -Zp -Wd-e68 & 145 & 550 & 666 \\
\hline Cray Y-MP M92 (1 proc. 6 ns) & CF77 5.0 -Zp -Wd-e68 & 145 & 332 & 333 \\
\hline Cray X-MP/416 (2 proc. 8.5 ns) & CF77 5.0 -Zp -Wd-e68 & 143 & 426 & 470 \\
\hline IBM RS/6000-R24 (71.5 MHz) & $\begin{array}{l}\text { v3.1.1 xlf -Pv -Wp,-me,-ew -O3 } \\
\text {-qarch=pwrx -qtune=pwrx -qhot-qhsflt } \\
\text {-qnosave }\end{array}$ & 142 & 246 & 284 \\
\hline DEC Alphastations $433 \mathrm{MHz}$ & $\mathrm{f} 90-\mathrm{O}$ & 141 & & 866 \\
\hline Hewlett-Packard C160 $160 \mathrm{MHz}$ & + Oall + Oinline $=$ daxpy & 140 & 421 & 640 \\
\hline IBM POWER2-990(71.5 MHz) & -O-Pv-Wp-ea478-g1-qarch=pwrx & 140 & 254 & 286 \\
\hline DEC 4100 5/300(4 proc, $300 \mathrm{MHz})$ & $\begin{array}{l}\text { kf77 -inline }=\text { daxpy }- \text { ur }=3 \text {-fast -O5 -tune } \\
\text { ev5 }\end{array}$ & & 1287 & 2400 \\
\hline DEC 4100 5/300(2 proc, $300 \mathrm{MHz})$ & $\begin{array}{l}\text { kf77 -inline }=\text { daxpy }- \text { ur }=3 \text {-fast }-05 \text {-tune } \\
\text { ev5 }\end{array}$ & & 734 & 1200 \\
\hline DEC 4100 5/300(1 proc, $300 \mathrm{MHz})$ & $\begin{array}{l}\text { kf77 -inline }=\text { daxpy }- \text { ur }=3 \text {-fast }-\mathrm{O} 5 \text {-tune } \\
\text { ev5 }\end{array}$ & 140 & 420 & 600 \\
\hline DEC $84005 / 350(8$ proc $350 \mathrm{MHz})$ & & & 2853 & 5600 \\
\hline DEC $84005 / 350(6$ proc $350 \mathrm{MHz})$ & & & 2313 & 4200 \\
\hline DEC 8400 5/350 (4 proc $350 \mathrm{MHz})$ & & & 1678 & 2800 \\
\hline DEC $84005 / 350(2$ proc $350 \mathrm{MHz})$ & & & 938 & 1400 \\
\hline DEC 8400 5/300 (1 proc $300 \mathrm{MHz})$ & -inline $=$ daxpy - ur $=3$-fast $-\mathrm{O} 5$-tune ev 5 & 140 & 411 & 600 \\
\hline DEC 8200 5/300 (6 proc 300 MHz) & & & 1821 & 3600 \\
\hline DEC 8200 5/300 (4 proc $300 \mathrm{MHz})$ & & & 1317 & 2400 \\
\hline DEC 8200 5/300 (2 proc 300 MHz) & & & 752 & 1200 \\
\hline
\end{tabular}




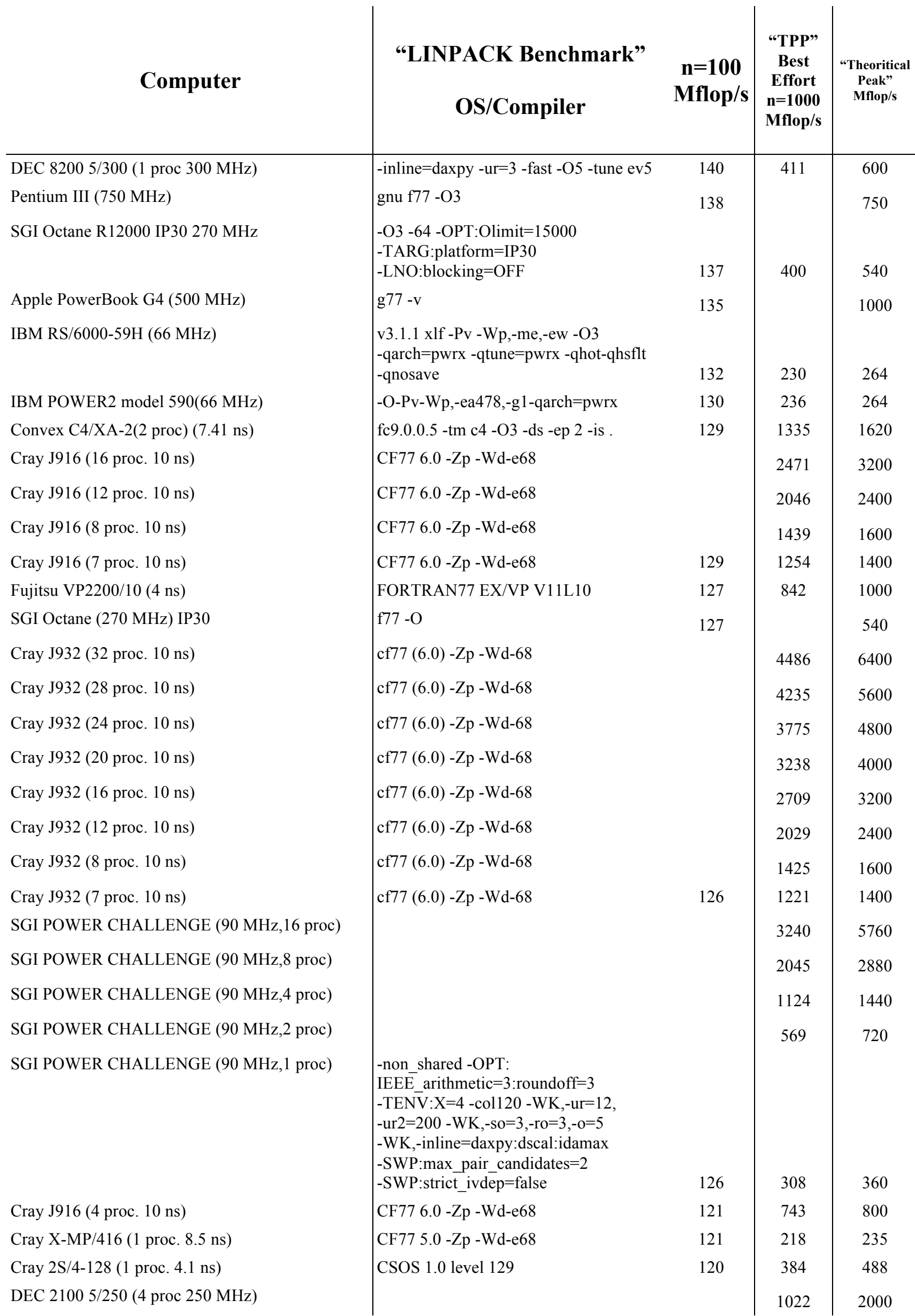




\begin{tabular}{|c|c|c|c|c|}
\hline Computer & $\begin{array}{c}\text { "LINPACK Benchmark" } \\
\text { OS/Compiler }\end{array}$ & $\begin{array}{c}\mathrm{n}=100 \\
\mathrm{Mflop} / \mathrm{s}\end{array}$ & $\begin{array}{c}\text { "TPP" } \\
\text { Best } \\
\text { Effort } \\
\text { n=1000 } \\
\text { Mflop/s }\end{array}$ & $\begin{array}{l}\text { "Theoritical } \\
\text { Peak" } \\
\text { Mflop/s }\end{array}$ \\
\hline DEC $21005 / 250(2$ proc $250 \mathrm{MHz})$ & & & 578 & 1000 \\
\hline DEC $21005 / 250(1$ proc $250 \mathrm{MHz})$ & -inline $=$ daxpy - ur $=3$-fast $-\mathrm{O} 5$-tune ev 5 & 119 & 317 & 500 \\
\hline Cray J932 (4 proc. $10 \mathrm{~ns})$ & cf77 (6.0) -Zp -Wd-68 & 117 & 730 & 800 \\
\hline ASUS P2B-D/PentiumIII(600 MHz,2 proc) & & & 745 & 1200 \\
\hline ASUS P2B-D/PentiumIII(600 MHz,1 proc) & ifc -tpp6 -O3 & 116 & 410 & 600 \\
\hline IBM RS/6000 F50 (332 MHz,4 proc) & & & 1049 & 2656 \\
\hline IBM RS/6000 F50 (332 MHz,3 proc) & & & 842 & 1992 \\
\hline IBM RS/6000 F50 (332 MHz,2 proc) & & & 599 & 1328 \\
\hline IBM RS/6000 F50 (332 MHz,1 proc) & $\begin{array}{l}\text {-O -qhot -qarch=ppc -qfloat=hsflt -Pv } \\
\text {-Wp,-ea478, -g1 -bnso } \\
\text {-bI:/lib/syscalls.exp -bnodelcsect }\end{array}$ & 116 & 317 & 664 \\
\hline SGI Origin 2000 (195 MHz, 16 proc) & & & 3146 & 6240 \\
\hline SGI Origin 2000 (195 MHz, 8 proc) & & & 2182 & 3120 \\
\hline SGI Origin 2000 (195 MHz, 4 proc) & & & 1292 & 1560 \\
\hline SGI Origin 2000 (195 MHz, 2 proc) & & & 667 & 780 \\
\hline SGI Origin 2000 (195MHz,1 proc) & $\begin{array}{l}\text {-n32 -mips4 -Ofast }=\text { ip } 27-T E N V: X=4 \\
\text {-LNO:blocking }=\text { off:ou_max }=6: p f 2=0\end{array}$ & 114 & 344 & 390 \\
\hline Sun UltraSparc II $250 \mathrm{MHz}$ & $\begin{array}{l}\text {-fast -native -xsafe }=\text { mem -dalign -xO5 } \\
\text {-xarch=v8plusa -xchip=ultra }\end{array}$ & 114 & 117 & 500 \\
\hline Fujitsu VP2100/10 (4 ns) & FORTRAN77 EX/VP V11L10 & 112 & 445 & 500 \\
\hline Cray J916 (2 proc. $10 \mathrm{~ns})$ & CF77 6.0 -Zp -Wd-e68 & 111 & 380 & 400 \\
\hline Sun Ultra HPC 6000(250 MHz,30 p) & & & 4755 & 15000 \\
\hline Sun Ultra HPC 6000(250 MHz,24 p) & & & 4389 & 12000 \\
\hline Sun Ultra HPC 6000(250 MHz,16 p) & & & 3493 & 8000 \\
\hline Sun Ultra HPC 6000(250 MHz,14 p) & & & 3112 & 7000 \\
\hline Sun Ultra HPC 6000(250 MHz, 8 p) & & & 2038 & 4000 \\
\hline Sun Ultra HPC 6000(250 MHz, 6 p) & & & 1607 & 3000 \\
\hline Sun Ultra HPC 6000(250 MHz, 4 p) & & & 1126 & 2000 \\
\hline Sun Ultra HPC 6000 (250 MHz,1MB L2) & $\begin{array}{l}\text {-fast -native -xarch=v8plusa -xsafe=mem } \\
\text {-dalign -libmil -xO5 -fsimple=2 } \\
\text {-stackvar -xarch=v8plusa } \\
\text {-xcache=16/32/1:512/64/1 -xchip=ultra } \\
\text {-xdepend -xlibmil -xlibmopt } \\
\text {-xsafe=mem -Qoption cg } \\
\text {-Qms_pipe+float_loop_ld=16 -xcrossfile }\end{array}$ & 110 & & 500 \\
\hline Cray J932 (2 proc. $10 \mathrm{~ns})$ & cf77 (6.0) -Zp -Wd-68 & 109 & 376 & 400 \\
\hline Hitachi S-820/80 (4 ns) & FORT77/HAP V23-0C & 107 & & 3000 \\
\hline Cray J916 (1 proc. $10 \mathrm{~ns})$ & CF77 6.0 -Zp -Wd-e68 & 106 & 203 & 200 \\
\hline Cray J932 (1 proc. $10 \mathrm{~ns})$ & cf77 (6.0) -Zp -Wd-68 & 104 & 202 & 200 \\
\hline Dell Dimension XPS T500 $500 \mathrm{MHz}$ & $\begin{array}{l}\text { Intel v5.0 -O3 -G6 -QxM -Qip -Qauto } \\
\text {-Qrcd }\end{array}$ & 104 & & 500 \\
\hline
\end{tabular}




\begin{tabular}{|c|c|c|c|c|}
\hline Computer & $\begin{array}{c}\text { "LINPACK Benchmark" } \\
\text { OS/Compiler }\end{array}$ & $\begin{array}{c}n=100 \\
\text { Mflop/s }\end{array}$ & $\begin{array}{c}\text { "TPP" } \\
\text { Best } \\
\text { Effort } \\
\text { n=1000 } \\
\text { Mflop/s }\end{array}$ & \begin{tabular}{|c} 
"Theoritical \\
Peak" \\
Mflop/s
\end{tabular} \\
\hline Cray $2 \mathrm{~S} / 8-128$ (8 proc. $4.1 \mathrm{~ns})$ & CF77 4.0 -Zp -Wd-e68 & 102 & 2171 & 3902 \\
\hline IBM POWER2 model 58H(55 MHz) & -O-Pv-Wp-ea478-g1-qarch=pwrx & 101 & 197 & 220 \\
\hline SGI POWER CHALLENGE (75 MHz,18 proc) & & & 3227 & 5400 \\
\hline SGI POWER CHALLENGE (75 MHz,16 proc) & & & 3033 & 4800 \\
\hline SGI POWER CHALLENGE (75 MHz,14 proc) & & & 2775 & 4200 \\
\hline SGI POWER CHALLENGE ( $75 \mathrm{MHz}, 12$ proc) & & & 2499 & 3600 \\
\hline SGI POWER CHALLENGE (75 MHz,10 proc) & & & 2167 & 3000 \\
\hline SGI POWER CHALLENGE ( $75 \mathrm{MHz}, 8$ proc) & & & 1818 & 2400 \\
\hline SGI POWER CHALLENGE ( $75 \mathrm{MHz}, 6$ proc) & & & 1421 & 1800 \\
\hline SGI POWER CHALLENGE ( $75 \mathrm{MHz}, 4$ proc) & & & 993 & 1200 \\
\hline SGI POWER CHALLENGE ( $75 \mathrm{MHz}, 2$ proc) & & & 505 & 600 \\
\hline SGI POWER CHALLENGE ( $75 \mathrm{MHz}, 1$ proc) & $\begin{array}{l}\text {-non_shared -OPT: } \\
\text { IEEE_arithmetic=3:roundoff=3 } \\
\text {-TENV:X=4 -col120 -WK,-ur=12, } \\
\text {-ur2=200 -WK,-so=3,-ro=3,-o=5 } \\
\text {-WK,-inline=daxpy:dscal:idamax } \\
\text {-SWP:max_pair_candidates }=2 \\
\text {-SWP:strict_ivdep=false }\end{array}$ & 104 & 261 & 300 \\
\hline Convex C4/XA-1(1 proc.)(7.41 ns) & fc9.0.0.5 -tm c4 - $\mathrm{O} 2$-is . & 99 & 705 & 810 \\
\hline Intel Pentium II Xeon (450 MHz) & g77 -funroll-all-loops -O3 & 98 & 295 & 450 \\
\hline ETA $10-\mathrm{G}$ (1 proc. $7 \mathrm{~ns})$ & ETAV/FTN200 & 93 & 496 & 571 \\
\hline Convex C-3880 (8 proc.) (16.7 ns) & fc7.0 -tm c38 -O3 -ep 8 -ds -is . & 86 & 795 & 960 \\
\hline IBM ES/9000-982 VF(8 proc 7.1ns) & VAST-2/VS Fortran V2R5 & & 2278 & 4507 \\
\hline IBM ES/9000-972 VF(7 proc 7.1ns) & VAST-2/VS Fortran V2R5 & & 2072 & 3944 \\
\hline IBM ES/9000-962 VF(6 proc 7.1ns) & VAST-2/VS Fortran V2R5 & & 1923 & 3380 \\
\hline IBM ES/9000-952 VF(5 proc $7.1 \mathrm{~ns})$ & VAST-2/VS Fortran V2R5 & & 1681 & 2817 \\
\hline IBM ES/9000-942 VF(4 proc 7.1ns) & VAST-2/VS Fortran V2R5 & & 1377 & 2254 \\
\hline IBM ES/9000-831 VF(3 proc 7.1ns) & VAST-2/VS Fortran V2R5 & & 1082 & 1690 \\
\hline IBM ES/9000-821 VF(2 proc $7.1 \mathrm{~ns})$ & VAST-2/VS Fortran V2R5 & & 767 & 1127 \\
\hline IBM ES/9000-711 VF(1 proc 7.1ns) & VAST-2/VS Fortran V2R5 & 86 & 422 & 563 \\
\hline Dell Dimension XPS T500(500 MHz) Pentium III & $\begin{array}{l}\text { Win98SE Intel Fortran -O3 -G6 -QaxK } \\
\text {-Qipo }\end{array}$ & 86 & & 500 \\
\hline Intel Pentium III $550 \mathrm{MHz}$ & $\begin{array}{l}\text { g77 -O3 -fomit-frame-pointer } \\
\text {-funroll-loops }\end{array}$ & 86 & 325 & 550 \\
\hline HALstation 300 model $350(118 \mathrm{MHz})$ & $\begin{array}{l}\text {-Kfast -Keval -KGREG -Kgs } \\
\text {-KV8PLUS -X7 -Kpreex -Kpreload } \\
\text {-Kfuse -x FLDFLAGS = -dn }\end{array}$ & 85 & 177 & 236 \\
\hline Dell Pentium III $550 \mathrm{MHz}$ & $\mathrm{f} 77-\mathrm{O} 3$ & 80 & & 550 \\
\hline SUN-Ultra 1 mod. 170 & f77 v4.0 -fast -O4 & 76 & & \\
\hline Convex C-3840 (4 proc.) (16.7 ns) & fc 7.0 -tm c38 -O3 -ep 4 -ds -is . & 75 & 425 & 480 \\
\hline Intel Pentium III $550 \mathrm{MHz}$ & g77 -O3 & 74 & 325 & 550 \\
\hline
\end{tabular}




\begin{tabular}{|c|c|c|c|c|}
\hline Computer & $\begin{array}{c}\text { "LINPACK Benchmark" } \\
\text { OS/Compiler }\end{array}$ & $\begin{array}{c}\mathbf{n}=\mathbf{1 0 0} \\
\mathbf{M f l o p} / \mathrm{s}\end{array}$ & $\begin{array}{c}\text { "TPP" } \\
\text { Best } \\
\text { Effort } \\
\text { n=1000 } \\
\text { Mflop/s }\end{array}$ & $\begin{array}{l}\text { "Theoritical } \\
\text { Peak" } \\
\text { Mflop/s }\end{array}$ \\
\hline HALstation 300 model $330(101 \mathrm{MHz})$ & $\begin{array}{l}\text {-Kfast -Keval -KGREG -Kgs } \\
\text {-KV8PLUS -X7 -Kpreex -Kpreload } \\
\text {-Kfuse -x FLDFLAGS = -dn }\end{array}$ & 72 & 153 & 202 \\
\hline SGI CHALLENGE/Onyx (6.6ns, 32 proc) & & & 539 & 2400 \\
\hline SGI CHALLENGE/Onyx (6.6ns, 28 proc) & & & 531 & 2100 \\
\hline SGI CHALLENGE/Onyx (6.6ns, 24 proc) & & & 499 & 1800 \\
\hline SGI CHALLENGE/Onyx (6.6ns, 20 proc) & & & 474 & 1500 \\
\hline SGI CHALLENGE/Onyx (6.6ns, 18 proc) & & & 458 & 1350 \\
\hline SGI CHALLENGE/Onyx (6.6ns, 16 proc) & & & 431 & 1200 \\
\hline SGI CHALLENGE/Onyx (6.6ns, 14 proc) & & & 393 & 1050 \\
\hline SGI CHALLENGE/Onyx (6.6ns, 12 proc) & & & 374 & 900 \\
\hline SGI CHALLENGE/Onyx (6.6ns, 10 proc) & & & 338 & 750 \\
\hline SGI CHALLENGE/Onyx (6.6ns, 8 proc) & $\begin{array}{l}\text { IRIX 5.2,f77,-O2-mips2-Wo, } \\
\text {-loopunroll,8-Olimit2000-Wf } \\
\text {-dchacheopt-jmpopt-non_shared -pfa } \\
\text { keep-WK, -WK, } \\
\text {-ipa=daxpy:saxpy,-ur=1,-mc=100 }\end{array}$ & 73 & 311 & 600 \\
\hline Convex C-3830 (3 proc.) (16.7 ns) & fc7.0 -tm c38 -O3 -ep 3 -ds -is . & 71 & 327 & 360 \\
\hline Sun UltraSPARC $1(24$ proc) $167 \mathrm{MHz}$ & & & 3566 & 8000 \\
\hline Sun UltraSPARC 1 (20 proc) $167 \mathrm{MHz}$ & & & 3170 & 6667 \\
\hline Sun UltraSPARC 1(16 proc)167MHz & & & 2761 & 5333 \\
\hline Sun UltraSPARC 1 (12 proc) $167 \mathrm{MHz}$ & & & 2238 & 4000 \\
\hline Sun UltraSPARC $1(8$ proc $) 167 \mathrm{MHz}$ & & & 1607 & 2667 \\
\hline Sun UltraSPARC 1(4 proc) $167 \mathrm{MHz}$ & & & 871 & 1333 \\
\hline Sun UltraSPARC 1 (2 proc) $167 \mathrm{MHz}$ & & & 456 & 667 \\
\hline Sun UltraSPARC 1 (1 proc) $167 \mathrm{MHz}$ & $\begin{array}{l}\text {-V -fast -native -dalign -libmil -xO4 } \\
\text {-xsafe=3Dmem -Qoption cg=20 } \\
\text {-Qms_pipe+float_loop_ld=3D16 -onetrip } \\
\text {-xcrossfile }\end{array}$ & 70 & 237 & 333 \\
\hline SGI CHALLENGE/Onyx (6.6ns, 6 proc) & $\begin{array}{l}\text { IRIX 5.2,f77,-O2-mips2-Wo, } \\
\text {-loopunroll,8-Olimit2000-Wf } \\
\text {-dchacheopt-jmpopt-non_shared -pfa } \\
\text { keep-WK, -WK, } \\
\text {-ipa=daxpy:saxpy,-ur=1,-mc=100 }\end{array}$ & 69 & & 450 \\
\hline Intel Pentium II, 333MHz & g77 -O3 -funroll-all-loops & 69 & & 333 \\
\hline AMD K6-2, $500 \mathrm{MHz}$ & $\begin{array}{l}\text { g77 -march=k6 -O3 -fomit-frame-pointer } \\
\text {-funroll-loops }\end{array}$ & 69 & 100 & 250 \\
\hline Convex SPP-1600(8 proc) $120 \mathrm{MHz}$ & & & 934 & 1920 \\
\hline Convex SPP-1200(8 proc) $120 \mathrm{MHz}$ & & & 656 & 1920 \\
\hline Convex SPP-1600(7 proc) $120 \mathrm{MHz}$ & & & 860 & 1680 \\
\hline Convex SPP-1600(6 proc) $120 \mathrm{MHz}$ & & & 722 & 1440 \\
\hline
\end{tabular}




\begin{tabular}{|c|c|c|c|c|}
\hline Computer & $\begin{array}{c}\text { "LINPACK Benchmark" } \\
\text { OS/Compiler }\end{array}$ & $\begin{array}{c}\mathrm{n}=100 \\
\mathrm{Mflop} / \mathrm{s}\end{array}$ & $\begin{array}{c}\text { "TPP" } \\
\text { Best } \\
\text { Effort } \\
\text { n=1000 } \\
\text { Mflop/s }\end{array}$ & $\begin{array}{l}\text { "Theoritical } \\
\text { Peak" } \\
\text { Mflop/s }\end{array}$ \\
\hline Convex SPP-1200(6 proc) $120 \mathrm{MHz}$ & & & 530 & 1440 \\
\hline Convex SPP-1600(5 proc) $120 \mathrm{MHz}$ & & & 633 & 1200 \\
\hline Convex SPP-1600(4 proc) $120 \mathrm{MHz}$ & & & 518 & 960 \\
\hline Convex SPP-1200(4 proc) $120 \mathrm{MHz}$ & & & 383 & 960 \\
\hline Convex SPP-1600(3 proc) $120 \mathrm{MHz}$ & & & 415 & 720 \\
\hline Convex SPP-1600(2 proc) $120 \mathrm{MHz}$ & & & 290 & 480 \\
\hline Convex SPP-1200(2 proc) $120 \mathrm{MHz}$ & & & 213 & 480 \\
\hline Convex SPP-1600(1 proc) $120 \mathrm{MHz}$ & $\mathrm{fc} 9.2 .1 \mathrm{fc}-$ is & 65 & 195 & 240 \\
\hline Convex SPP-1200(1 proc) $120 \mathrm{MHz}$ & $\mathrm{fc} 9.2 .1 \mathrm{fc}-$ is & 65 & 123 & 240 \\
\hline Dell Pentium III $450 \mathrm{MHz}$ & $\mathrm{f} 77-\mathrm{O} 3$ & 65 & & 450 \\
\hline SUN-Ultra 1 mod. 140 & f77 v4.0 -fast -O4 & 63 & & \\
\hline Convex C-3820 (2 proc.) (16.7 ns) & fc7.0 -tm c38-O3 -ep 2 -ds -is . & 62 & 222 & 240 \\
\hline AMD K6-II (350 MHz) BCM-1541 ATX & g77 -O3 -o g77ldst & 64 & & 350 \\
\hline Cray-2/4-256 (4 proc. $4.1 \mathrm{~ns})$ & $\operatorname{cf} 773.0$ & 62 & 1226 & 1951 \\
\hline ETA 10 -E (1 proc. $10.5 \mathrm{~ns})$ & ETAV/FTN200 & 62 & 334 & 381 \\
\hline Gateway 2000 G6-200 PentiumPro & MS Fortran NT /G5 /Oxb2 & 62 & & 200 \\
\hline IBM ES/9000-900 VF(6 proc. 9 ns) & VAST-2/VS Fortran V2R4 & & 1457 & 2664 \\
\hline IBM ES/9000-860 VF(5 proc. 9 ns) & VAST-2/VS Fortran V2R4 & & 1210 & 2220 \\
\hline IBM ES/9000-820 VF(4 proc. 9 ns) & VAST-2/VS Fortran V2R4 & & 1003 & 1776 \\
\hline IBM ES/9000-740 VF(3 proc. 9 ns) & VAST-2/VS Fortran V2R4 & & 775 & 1332 \\
\hline IBM ES/9000-640 VF(2 proc. 9 ns) & VAST-2/VS Fortran V2R4 & & 539 & 888 \\
\hline IBM ES/9000-660 VF(2 proc. 9 ns) & VAST-2/VS Fortran V2R4 & & 535 & 888 \\
\hline IBM ES/9000-520 VF(1 proc. 9 ns) & VAST-2/VS Fortran V2R4 & 60 & 338 & 444 \\
\hline SGI CHALLENGE/Onyx (6.6ns, 4 proc) & $\begin{array}{l}\text { IRIX 5.2,f77,-O2-mips2-Wo, } \\
\text {-loopunroll,8-Olimit2000-Wf } \\
\text {-dchacheopt-jmpopt-non_shared -pfa } \\
\text { keep-WK, -WK, } \\
\text {-ipa=daxpy:saxpy,-ur=1,-mc=100 }\end{array}$ & 58 & 178 & 300 \\
\hline Cray X-MP/14se (10 ns) & $\operatorname{cf77} 3.0$ & 53 & 184 & 210 \\
\hline DEC $7000-760$ (6 proc) $3.64 \mathrm{~ns}$ & & & 962 & 1650 \\
\hline DEC $7000-740$ (4 proc) $3.64 \mathrm{~ns}$ & & & 693 & 1100 \\
\hline DEC $7000-720$ (2 proc) $3.64 \mathrm{~ns}$ & & & 361 & 550 \\
\hline DEC $7000-710$ (1 proc) $3.64 \mathrm{~ns}$ & 3.6 -O5 -fast & 53 & 208 & 275 \\
\hline IBM RS/6000-390 (66.5 MHz) & $\begin{array}{l}\text { v3.1.1 xlf -Pv -Wp,-fz,-me,-ew -O3 -Q } \\
\text {-qstrict -qarch=pwr-qtune =pwrx -qhot } \\
\text {-qhsflt -qnosave }\end{array}$ & 53 & 181 & 266 \\
\hline DEC 2100 4/275 A500MP(4 proc) & & & 625 & 1100 \\
\hline DEC 2100 4/275 A500MP(2 proc) & & & 348 & 550 \\
\hline DEC 2100 4/275 A500MP(1 proc) & 3.6 -O5 -fast & 52 & 208 & 275 \\
\hline
\end{tabular}




\begin{tabular}{|c|c|c|c|c|}
\hline Computer & $\begin{array}{c}\text { "LINPACK Benchmark" } \\
\text { OS/Compiler }\end{array}$ & $\begin{array}{c}\mathrm{n}=100 \\
\mathrm{Mfl} / \mathrm{pp} / \mathrm{s}\end{array}$ & $\begin{array}{c}\text { "TPP" } \\
\text { Best } \\
\text { Effort } \\
\text { n=1000 } \\
\text { Mflop/s }\end{array}$ & \begin{tabular}{|} 
"Theoritical \\
Peak" \\
Mflop/s
\end{tabular} \\
\hline DEC $3000-900$ (1 proc) $3.64 \mathrm{~ns}$ & 3.6 -O5 -fast & 52 & 193 & 275 \\
\hline AMD K6-II $500 \mathrm{Mhz}$ & g77 -O3 & 51 & & 500 \\
\hline Convex SPP-1000(15 procs)100MHz & & & 965 & 3000 \\
\hline Convex SPP-1000(12 procs) $100 \mathrm{MHz}$ & & & 916 & 2400 \\
\hline Convex SPP-1000(8 procs) $100 \mathrm{MHz}$ & & & 751 & 1600 \\
\hline Convex SPP-1000(4 procs) $100 \mathrm{MHz}$ & & & 442 & 800 \\
\hline Convex SPP-1000(2 procs) $100 \mathrm{MHz}$ & & & 255 & 400 \\
\hline Convex SPP-1000(1 proc) $100 \mathrm{MHz}$ & fc9.2.1 fc -is & 48 & 123 & 200 \\
\hline Cray-2/4-256 (2 proc. $4.1 \mathrm{~ns})$ & $\operatorname{cf} 773.0$ & 48 & 709 & 976 \\
\hline IBM ES/9000-711 (1 proc 7.1ns) & VAST-2/VS Fortran V2R5 & 48 & & \\
\hline DEC $3000-700$ (1 proc) $4.44 \mathrm{~ns}$ & 3.6 -O5 -fast & 45 & 164 & 225 \\
\hline DEC 400 4/233 (1 proc) $4.3 \mathrm{~ns}$ & 3.6 -O5 -fast & 45 & 138 & 233 \\
\hline Compaq/DEC Alpha 21164 EV56 533 MHz & g77 -O3 & 45 & 501 & 1066 \\
\hline Convex C-3810 (1 proc.) (16.7 ns) & fc7.0 -tm c38 -O2 -is . & 44 & 113 & 120 \\
\hline DEC $7000-660$ (6 procs) $5.0 \mathrm{~ns}$ & & & 755 & 1200 \\
\hline DEC $7000-650$ (5 procs) $5.0 \mathrm{~ns}$ & & & 641 & 1000 \\
\hline DEC $7000-640$ (4 procs) $5.0 \mathrm{~ns}$ & & & 538 & 800 \\
\hline DEC $7000-630$ (3 procs) $5.0 \mathrm{~ns}$ & & & 413 & 600 \\
\hline DEC $7000-620$ ( 2 procs) $5.0 \mathrm{~ns}$ & & & 279 & 400 \\
\hline DEC $7000-610$ (1 proc) $5.0 \mathrm{~ns}$ & 1.3 -O5 -fast & 44 & 156 & 200 \\
\hline DEC 3000-800 Alpha AXP $5.0 \mathrm{~ns}$ & 1.3 -O5 -fast & 44 & 145 & 200 \\
\hline DEC 2100-A500MP(4 procs $) 5.25 \mathrm{~ns}$ & 1.3 -O5 -fast & & 358 & 760 \\
\hline DEC $2100-\mathrm{A} 500 \mathrm{MP}(3$ procs $) 5.25 \mathrm{~ns}$ & 1.3 -O5 -fast & & 293 & 570 \\
\hline DEC 2100-A500MP(2 procs $) 5.25 \mathrm{~ns}$ & 1.3 -O5 -fast & & 209 & 380 \\
\hline DEC $2100-A 500 \mathrm{MP}(1$ proc) $5.25 \mathrm{~ns}$ & 1.3 -O5 -fast & 43 & 129 & 190 \\
\hline DEC 10000-660 Alpha AXP(6 proc) & & & 751 & 1200 \\
\hline DEC $10000-650$ Alpha AXP(5 proc $)$ & & & 639 & 1000 \\
\hline DEC 10000-640 Alpha AXP(4 proc) & & & 523 & 800 \\
\hline DEC 10000-630 Alpha AXP(3 proc) & & & 403 & 600 \\
\hline DEC 10000-620 Alpha AXP(2 proc) & & & 273 & 400 \\
\hline DEC 10000-610 Alpha AXP $200 \mathrm{MHz}$ & $3.2 \mathrm{inl}=$ daxpy, ur $=4$, ur2 $=240$ & 43 & 155 & 200 \\
\hline NEC SX-2 (6 ns) & FORTRAN 77/SX & 43 & 885 & 1300 \\
\hline Cray Y-MP EL (4 proc. 30 ns) & CF77 5.0 -Zp -Wd-e68 & 41 & 345 & 532 \\
\hline HP 9000/735 (99 MHz) & $\begin{array}{l}+\mathrm{OP} 3 \text {-W1,-aarchive -WP,-nv -w, } \\
\text { ConvexMLIB } 1.2\end{array}$ & 41 & 120 & 198 \\
\hline Compaq Proliant 5000200 MHz & MS Power Stat. 4.0 Full Opt & 40 & & 200 \\
\hline Cray Y-MP EL98 (8 proc. 30 ns) & CF77 5.0 -Zp -Wd-e68 & 40 & 567 & 1068 \\
\hline
\end{tabular}




\begin{tabular}{|c|c|c|c|c|}
\hline Computer & $\begin{array}{c}\text { "LINPACK Benchmark" } \\
\text { OS/Compiler }\end{array}$ & $\begin{array}{c}\mathrm{n}=100 \\
\mathrm{Mflop} / \mathrm{s}\end{array}$ & $\begin{array}{c}\text { "TPP" } \\
\text { Best } \\
\text { Effort } \\
\text { n=1000 } \\
\text { Mflop/s }\end{array}$ & $\begin{array}{c}\text { "Theoritical } \\
\text { Peak" } \\
\text { Mflop/s }\end{array}$ \\
\hline Cray Y-MP EL98 (4 proc. 30 ns) & CF77 5.0-Zp -Wd-e68 & 40 & 357 & 534 \\
\hline Cray Y-MP EL94 (4 proc. 30 ns) & CF77 5.0 -Zp -Wd-e68 & 40 & 331 & 532 \\
\hline Cray S-MP/11v2 (1 proc. 30 ns) & uf77 5.1.2 vec=collapse pi + & 39 & 206 & 267 \\
\hline Cray Y-MP EL94 (2 proc. 30 ns) & CF77 5.0-Zp -Wd-e68 & 39 & 190 & 266 \\
\hline Cray Y-MP EL (2 proc. $30 \mathrm{~ns}$ ) & CF77 5.0 -Zp -Wd-e68 & 39 & 191 & 266 \\
\hline DEC $4000-720$ (2 procs) $5.25 \mathrm{~ns}$ & & & 235 & 380 \\
\hline DEC $4000-710$ (1 procs) $5.25 \mathrm{~ns}$ & 1.3 -O5 -fast & 39 & 143 & 190 \\
\hline DEC 1000 4/200 (5 ns) & $3.6-\mathrm{O} 5$-fast & 39 & 147 & 200 \\
\hline HP9000/J200 (100 MHz) & +O3 +DC7200 +Odataprefetch & 38 & & \\
\hline Cray-2/4-256 (1 proc. $4.1 \mathrm{~ns})$ & cf77 3.0 & 38 & 360 & 488 \\
\hline IBM RISC Sys/6000-580 (62.5MHz) & v2.3 xlf -O -P -Wp,-ea478 & 38 & 104 & 125 \\
\hline IBM RISC Sys/6000-980 (62.5MHz) & v2.3 xlf -O -P -Wp,-ea478 & 38 & 104 & 125 \\
\hline IBM ES/9000-520 (1 proc. 9 ns) & VAST-2/VS Fortran V2R4 & 38 & & \\
\hline IBM ES/9000-820 (1 proc. 9 ns) & VAST-2/VS Fortran V2R4 & 38 & & \\
\hline SGI CHALLENGE/Onyx (6.6ns, 2 proc) & $\begin{array}{l}\text { IRIX 5.2,f77,-O2-mips2-Wo, } \\
\text {-loopunroll,8-Olimit2000-Wf } \\
\text {-dchacheopt-jmpopt-non_shared -pfa } \\
\text { keep-WK, -WK, } \\
\text {-ipa=daxpy:saxpy,-ur=1,-mc=100 }\end{array}$ & 38 & 93.5 & 150 \\
\hline DEC 4000-610 Alpha AXP(160 MHz) & $3.2 \mathrm{inl}=$ daxpy,ur= $=4, \mathrm{ur} 2=240$ & 36 & 114 & 160 \\
\hline Pentium Pro $200 \mathrm{Mhz}$ & Solaris 2.5 GNU F77 v0.5.5 & 38 & & 200 \\
\hline NEC SX-1 & FORTRAN 77/SX & 36 & 422 & 650 \\
\hline Cray Y-MP EL98 (2 proc. 30 ns) & CF77 5.0 -Zp -Wd-e68 & 35 & 192 & 267 \\
\hline Apple Macintosh 9500/233 & MF -O4 -Asched $=2, \operatorname{targ}=604$ & 34 & & \\
\hline Apple Macintosh 6500/275 & MF -O4 -Asched $=2, \operatorname{targ}=604$ & 20 & & \\
\hline Convex C-3440 (4 proc.) & fc7.0 fc -O3 -ep 4 -ds -is . & 34 & 172 & 200 \\
\hline Cray Y-MP EL98 (1 proc. 30 ns) & CF77 5.0-Zp -Wd-e68 & 34 & 107 & 133 \\
\hline ETA $10-\mathrm{Q}$ (1 proc. $19 \mathrm{~ns})$ & ETAV/FTN200 & 34 & 185 & 210 \\
\hline Cray Y-MP EL94 (1 proc. 30 ns) & CF77 5.0 -Zp -Wd-e68 & 34 & 107 & 133 \\
\hline Cray Y-MP EL (1 proc. $30 \mathrm{~ns}$ ) & CF77 5.0 -Zp -Wd-e68 & 34 & 107 & 133 \\
\hline DEC 3000-600 Alpha AXP 5.7 ns & 1.3 -O5 -fast & 34 & 129 & 180 \\
\hline Cray S-MP/MCP784(84 proc. 25 ns) & & & 742 & 3360 \\
\hline Cray S-MP/MCP756(56 proc. 25 ns) & & & 678 & 2240 \\
\hline Cray S-MP/MCP728(28 proc. 25 ns) & & & 508 & 1120 \\
\hline Cray S-MP/MCP707 (7 proc. 25 ns) & MCP Release 2.2 & 33 & 194 & 280 \\
\hline DEC $2004 / 166$ (1 proc) $6 \mathrm{~ns}$ & $3.6-\mathrm{O} 5$-fast & 33 & 100 & 167 \\
\hline FPS 510S MCP784 (84 proc. 25 ns) & & & 548 & 3360 \\
\hline FPS 510S MCP756 (56 proc. 25 ns) & & & 513 & 2240 \\
\hline FPS 510S MCP728 (28 proc. 25 ns) & & & 414 & 1120 \\
\hline FPS 510S MCP707 (7 proc. $25 \mathrm{~ns}$ ) & pgf77 -O4 -Minline & 33 & 184 & 280 \\
\hline
\end{tabular}




\begin{tabular}{|c|c|c|c|c|}
\hline Computer & $\begin{array}{c}\text { "LINPACK Benchmark" } \\
\text { OS/Compiler }\end{array}$ & $\begin{array}{c}n=100 \\
\text { Mflop/s }\end{array}$ & $\begin{array}{c}\text { "TPP" } \\
\text { Best } \\
\text { Effort } \\
\text { n=1000 } \\
\text { Mflop/s }\end{array}$ & \begin{tabular}{|c} 
"Theoritical \\
Peak" \\
Mflop/s
\end{tabular} \\
\hline CDC Cyber $2000 \mathrm{~V}$ & Fortran V2 & 32 & & \\
\hline Convex C-3430 (3 proc.) & fc 7.0 fc -03 -ep 3 -ds -is. & 32 & 132 & 150 \\
\hline Macintosh 7300/200MHz & 4.4, Absoft Corp.-c -O -o & 32 & & 200 \\
\hline NEC SX-1E & FORTRAN 77/SX & 32 & 221 & 325 \\
\hline SGI Indigo2 (R4400/200MHz) & $\begin{array}{l}\text {-mips2 -Olimit } 3000 \text {-Wo, -loopunroll, } 8 \\
\text {-Wf,-dcacheopt -Wf,-dcacheoptx -O3 } \\
\text {-non_shared }\end{array}$ & 32 & & \\
\hline Alliant FX/2800-200 (14 proc) & fortran 1.1.27 -O -inline & 31 & 325 & 560 \\
\hline IBM RISC Sys/6000-970 (50 MHz) & v2.2.1 xlf -O -P -Wp,-ea478 & 31 & 84 & 100 \\
\hline IBM RS/6000 Cluster(8 proc $62.5 \mathrm{MHz})$ & & & 269 & 1000 \\
\hline IBM RS/6000 Cluster(4 proc $62.5 \mathrm{MHz}$ ) & & & 206 & 500 \\
\hline IBM RS/6000 Cluster(2 proc $62.5 \mathrm{MHz}$ ) & & & 144 & 250 \\
\hline IBM RS/6000 Cluster(8 proc $50 \mathrm{MHz}$ ) & & & 194 & 800 \\
\hline IBM RS/6000 Cluster(6 proc $50 \mathrm{MHz}$ ) & & & 174 & 600 \\
\hline IBM RS/6000 Cluster(4 proc $50 \mathrm{MHz}$ ) & & & 152 & 400 \\
\hline IBM RS/6000 Cluster(2 proc $50 \mathrm{MHz}$ ) & & & 111 & 200 \\
\hline IBM RISC Sys/6000-560 (50 MHz) & v2.2.1 xlf -O -P -Wp,-ea478 & 31 & 84 & 100 \\
\hline IBM ES/9000-742 VF(4 proc 11ns) & VAST-2/VS Fortran V2R5 & & 441 & 752 \\
\hline IBM ES/9000-732 VF(3 proc 11ns) & VAST-2/VS Fortran V2R5 & & 352 & 545 \\
\hline IBM ES/9000-622 VF(2 proc $11 \mathrm{~ns})$ & VAST-2/VS Fortran V2R5 & & 244 & 364 \\
\hline IBM ES/9000-621 VF(2 proc 11ns) & VAST-2/VS Fortran V2R5 & & 244 & 364 \\
\hline IBM ES/9000-521 VF(2 proc $11 \mathrm{~ns})$ & VAST-2/VS Fortran V2R5 & & 185 & 364 \\
\hline IBM ES/9000-511 VF(1 proc $11 \mathrm{~ns})$ & VAST-2/VS Fortran V2R5 & 30 & 130 & 182 \\
\hline DEC 3000-500 Alpha AXP(150 MHz) & $3.2 \mathrm{inl}=$ daxpy,ur $=4, \mathrm{ur} 2=240$ & 30 & 107 & 150 \\
\hline Hitachi SR2201(1 proc $150 \mathrm{MHz})$ & f90 PVEC,OPT(0(S),FOLD(2)) & 30 & 248 & 300 \\
\hline SGI CHALLENGES 200Mhz R4400SC & IRIX 5.3 f77 -O4 -mips2 & 30 & & \\
\hline Alliant FX/2800-200 (12 proc) & fortran 1.1.27 -O -inline & 29 & 290 & 480 \\
\hline HP 9000/715 (75 MHz) & HP-UX f77 +OP4 & 29 & & \\
\hline IBM 9672-R12 & VAST-2/VS Fortran 2.5 & 29 & & 83 \\
\hline Sun Sparc 2090 MHz, (1 proc) & Sun 5.3 -fast -unroll=4 -O4 & 29 & & \\
\hline Intel Pentium $166 \mathrm{MHz}$ & ifc -O3 -ip -align & 28.37 & 79.37 & 166 \\
\hline Alliant FX/2800-200 (10 proc) & fortran 1.1.27 -O -inline & 27 & 250 & 400 \\
\hline ETA $10-\mathrm{P}$ (1 proc. $24 \mathrm{~ns})$ & ETAV/FTN200 & 27 & 146 & 167 \\
\hline Convex C-3420 (2 proc.) & fc 7.0 fc -O3 -ep 2 -ds -is . & 27 & 90 & 100 \\
\hline Cray-1S (12.5 ns) & cf77 2.1 & 27 & 110 & 160 \\
\hline Convex C-3240 (4 proc.) & fc $-\mathrm{O} 3$-ep 2 -uo -pp=fcpp 1 -is . & 26 & 171 & 200 \\
\hline Convex C-240 (4 proc.) & $6.1-\mathrm{O} 3-\mathrm{ep} 2-$-uo $-\mathrm{pp}=\mathrm{fcpp} 1$-is . & 26 & 166 & 200 \\
\hline Convex C-3230 (3 proc.) & fc $-\mathrm{O} 3$-ep 2 -uo -pp=fcpp 1 -is . & 26 & 132 & 150 \\
\hline
\end{tabular}




\begin{tabular}{|c|c|c|c|c|}
\hline Computer & $\begin{array}{c}\text { "LINPACK Benchmark" } \\
\text { OS/Compiler }\end{array}$ & $\begin{array}{c}n=100 \\
\text { Mflop/s }\end{array}$ & $\begin{array}{c}\text { "TPP" } \\
\text { Best } \\
\text { Effort } \\
\text { n=1000 } \\
\text { Mflop/s }\end{array}$ & $\begin{array}{l}\text { "Theoritical } \\
\text { Peak" } \\
\text { Mflop/s }\end{array}$ \\
\hline Convex C-230 (3 proc.) & $6.1-\mathrm{O} 3$-ep2 -uo -pp=fcpp1 -is . & 26 & 128 & 150 \\
\hline DEC 2000-300 Alpha AXP $6.7 \mathrm{~ns}$ & 1.3 -O5 -fast & 26 & 88 & 150 \\
\hline DEC 3000-400 Alpha AXP(133 MHz) & 3.2 inl $=$ daxpy,ur $=4, u r 2=240$ & 26 & 90 & 133 \\
\hline IBM RISC Sys/6000-950 (42 MHz) & v2.2.1 xlf -O -P -Wp,-ea478 & 26 & 70 & 84 \\
\hline IBM RISC Sys/6000-550 (42 MHz) & v2.2.1 xlf -O -P -Wp,-ea478 & 26 & 70 & 84 \\
\hline IBM RISC Sys/6000-375(62.5 MHz) & v2.3.0 xlf -O -P -Wp,-ea478 & 26 & 90 & 125 \\
\hline IBM RISC Sys/6000-370(62.5 MHz) & v2.3.0 xlf -O -P -Wp,-ea478 & 26 & 90 & 125 \\
\hline SGI CHALLENGE/Onyx (6.6ns, 1 proc) & $\begin{array}{l}\text { IRIX 5.2,f77,-O2-mips2-Wo, } \\
\text {-loopunroll,8-Olimit2000-Wf } \\
\text {-dchacheopt-jmpopt-non_shared -pfa } \\
\text { keep-WK, -WK, } \\
\text {-ipa=daxpy:saxpy,-ur=1,-mc=100 }\end{array}$ & 26 & 48.4 & 75 \\
\hline Alliant FX/2800 210 (1 proc) & fortran $1.3 .02-\mathrm{Ovg}$-inline & 25 & 34 & 50 \\
\hline Alliant FX/2800-200 (8 proc) & fortran 1.1.27 -O -inline & 25 & 207 & 320 \\
\hline NAS AS/EX 100 VPF (4 proc) & & & 320 & 484 \\
\hline NAS AS/EX 90 VPF (3 proc) & & & 251 & 363 \\
\hline NAS AS/EX 80 VPF (2 proc) & & & 173 & 242 \\
\hline NAS AS/EX $60 \mathrm{VPF}$ & VAST-2/VS $2.3 .0 \mathrm{opt}=3$ & 25 & 94 & 121 \\
\hline HP 9000/750 (66 MHz) & +OP3 -W1,-aarchive -WP,-nv -w & 24 & 47 & 66 \\
\hline HP 9000/730 (66 MHz) & +OP3 -W1,-aarchive -WP,-nv -w & 24 & 49 & 66 \\
\hline IBM ES/9000 Model 480 VF & VAST-2/VS Fortran V2R4 & & 180 & 266 \\
\hline IBM ES/9000-340 VF (14.5 ns) & VAST-2/VS Fortran V2R4 & 23 & & 138 \\
\hline IBM ES/9000-411 VF(1 proc $11 \mathrm{~ns})$ & VAST-2/VS Fortran V2R5 & 23 & 99 & 182 \\
\hline Meiko CS2 (64 proc) & & & 652 & 11520 \\
\hline Meiko CS2 (32 proc) & & & 649 & 5760 \\
\hline Meiko CS2 (16 proc) & & & 530 & 2880 \\
\hline Meiko CS2 (8 proc) & & & 420 & 1440 \\
\hline Meiko CS2 (4 proc) & & & 289 & 720 \\
\hline Meiko CS2 (2 proc) & & & 169 & 360 \\
\hline Meiko CS2 (1 proc) & -dalign $-\mathrm{O} 5-\mathrm{XT}=\mathrm{ss} 10 \mathrm{~h}$, unroll $=1$ & 24 & 97 & 180 \\
\hline Fujitsu M1800/20 & EX V10L20 frt -Of -Ne & 23 & & \\
\hline Intel Pentium $166 \mathrm{MHz}$ & $\begin{array}{l}\text { g77 -march=pentium -O3 } \\
\text {-fomit-frame-pointer -funroll-loops }\end{array}$ & 23 & 78 & 166 \\
\hline Sun Sparc 10-52 (1 proc) & Sun 3.0 -fast -O4 -unroll=4 -Bstatic & 23 & & \\
\hline DEC VAX $9000420 \mathrm{VP}(2$ proc $16 \mathrm{~ns})$ & HPO V1.3-163V, DXML & & 155 & 250 \\
\hline DEC VAX $9000410 \mathrm{VP}(1$ proc $16 \mathrm{~ns})$ & HPO V1.3-163V, DXML & 22 & 89 & 125 \\
\hline IBM ES/9000-610 VF (4 proc $15 \mathrm{~ns}$ ) & VAST-2/VS Fortran V2R4 & & 335 & 532 \\
\hline IBM ES/9000-570 VF (3 proc 15 ns) & VAST-2/VS Fortran V2R4 & & 252 & 399 \\
\hline Apple Macintosh 9500/132 & MF77 -O4 -Ashed $=2$, target $=604$ & 22 & & \\
\hline
\end{tabular}




\begin{tabular}{|c|c|c|c|c|}
\hline Computer & $\begin{array}{c}\text { "LINPACK Benchmark" } \\
\text { OS/Compiler }\end{array}$ & $\begin{array}{c}n=100 \\
\text { Mflop/s }\end{array}$ & $\begin{array}{c}\text { "TPP" } \\
\text { Best } \\
\text { Effort } \\
\text { n=1000 } \\
\text { Mflop/s }\end{array}$ & $\begin{array}{l}\text { "Theoritical } \\
\text { Peak" } \\
\text { Mflop/s }\end{array}$ \\
\hline IBM ES/9000-490 VF (2 proc $15 \mathrm{~ns})$ & VAST-2/VS Fortran V2R4 & & 171 & 266 \\
\hline IBM ES/9000-320 VF (1 proc $15 \mathrm{~ns})$ & VAST-2/VS Fortran V2R4 & 22 & 91 & 133 \\
\hline IBM RISC Sys/6000-570 (50 MHz) & v2.3.0 xlf -O -P -Wp,-ea478 & 22 & 73 & 100 \\
\hline IBM RISC Sys/6000-365 (50 MHz) & v2.3.0 xlf -O -P -Wp,-ea478 & 22 & 73 & 100 \\
\hline IBM RISC Sys/6000-360 (50 MHz) & v2.3.0 xlf -O -P -Wp,-ea478 & 22 & 73 & 100 \\
\hline Multiflow TRACE 28/300 & Fortran 2.2 .1 & 22 & 69 & 123 \\
\hline Convex C-3220 (2 proc.) & fc $-\mathrm{O} 3$-ep 2 -uo $-\mathrm{pp}=\mathrm{fcpp} 1$-is . & 22 & 89 & 100 \\
\hline Convex C-220 (2 proc.) & $6.1-\mathrm{O} 3-\mathrm{ep} 2-$-uo $-\mathrm{pp}=\mathrm{fcpp} 1-$ is . & 22 & 87 & 100 \\
\hline Alliant FX/2800-200 (6 proc) & fortran 1.1.27 -O -inline & 21 & 148 & 240 \\
\hline Siemens VP400-EX (7 ns) & Fortran 77/VP V10L30 & 21 & 794 & 1714 \\
\hline IBM ES/9221-211 (16 ns) & VAST-2/VS Fortran 2.5 & 21 & & \\
\hline Apple Macintosh 6500/275 & MF -O4 -Asched $=2, \operatorname{targ}=604$ & 20 & & \\
\hline Apple Power Mac 8500/120 & Absoft Power PC v4.1 -O -U & 20 & & \\
\hline FPS Model 522 & F77 4.2 & 20 & 105 & 133 \\
\hline Fujitsu VP-400 & Fortran 77 V10L30 & 20 & & 1142 \\
\hline IBM RISC Sys/6000-530H(33 MHz) & v2.2.1 xlf -O -P -Wp,-ea478 & 20 & 55 & 66 \\
\hline IBM RS/6000-C10(601 - $80 \mathrm{MHz})$ & $\begin{array}{l}\text { v3.1.1 xlf -Pv -Wp,-fz,-me, -ew -O3 } \\
\text {-qarch=ppc -qhot -qhsflt -qnosave } \\
\text {-qnofold }\end{array}$ & 20 & 63 & 80 \\
\hline IBM ES/9672-R11 (16 ns) & VAST-2/VS Fortran 2.5 & 20 & & \\
\hline Siemens VP200-EX (7 ns) & Fortran 77 V10L30 & 20 & 472 & 857 \\
\hline Amdahl 1400 & 77/VP V10L20 & 19 & 521 & 1142 \\
\hline Amdahl 1200 & 77/VP V10L20 & 19 & 424 & 571 \\
\hline Apple Power Mac 9500/132 & Absoft Power PC v4.1 -O -U & 19 & & \\
\hline Convex C-3410 (1 proc.) & fc $7.0 \mathrm{fc}-\mathrm{O} 2$-is . & 19 & 47 & 50 \\
\hline Gateway 2000 P5-133 & MS PS 32 NT /G5 /Oxb2 & 19 & & \\
\hline IBM ES/9000 Model 260 VF (15 ns) & VAST-2/VS Fortran V2R4 & 19 & 78 & 133 \\
\hline IBM RISC Sys/6000-550L(42 MHz) & v2.3.0 xlf -O -P -Wp,-ea478 & 19 & 61 & 82 \\
\hline IBM RISC Sys/6000-540 (30 MHz) & v2.2.1 xlf -O -P -Wp,-ea478 & 19 & 50 & 60 \\
\hline IBM RISC Sys/6000-355 (42 MHz) & v2.3.0 xlf -O -P -Wp,-ea478 & 19 & 61 & 84 \\
\hline IBM RISC Sys/6000-350 (42 MHz) & v2.2.1 xlf -O -P -Wp,-ea478 & 19 & 61 & 84 \\
\hline IBM RISC Sys/6000-34H (42 MHz) & v2.3.0 xlf -O -P -Wp,-ea478 & 19 & 61 & 84 \\
\hline IBM ES/9000-311 VF(1 proc $11 \mathrm{~ns})$ & VAST-2/VS Fortran V2R5 & 19 & 82 & 182 \\
\hline Cray S-MP/11 (1 proc. $30 \mathrm{~ns})$ & uf77 5.1.2 -Oc a2 & 18 & 60 & 67 \\
\hline Compaq Deskpro $4000166 \mathrm{MHz}$ & MS Power Stat. 4.0 Full Opt & 18 & & 166 \\
\hline Fujitsu VP-200 & Fortran 77 & 18 & 422 & 533 \\
\hline HP 9000/720 (50 MHz) & HP-UX 8.05 f77 +OP4 +O3 & 18 & 36 & 50 \\
\hline IBM ES/9221-201 (16 ns) & VAST-2/VS Fortran 2.5 & 18 & & \\
\hline NAS AS/EX $50 \mathrm{VPF}$ & VAST-2/VS 2.3 .0 & 18 & 82 & 121 \\
\hline SGI $4 \mathrm{D} / 480(8$ proc $) 40 \mathrm{MHz}$ & $\mathrm{f} 77-\mathrm{O} 2-\mathrm{mp}$ & 18 & 71 & 128 \\
\hline
\end{tabular}




\begin{tabular}{|c|c|c|c|c|}
\hline Computer & $\begin{array}{c}\text { "LINPACK Benchmark" } \\
\text { OS/Compiler }\end{array}$ & $\begin{array}{c}n=100 \\
\text { Mflop/s }\end{array}$ & $\begin{array}{c}\text { "TPP" } \\
\text { Best } \\
\text { Effort } \\
\text { n=1000 } \\
\text { Mflop/s }\end{array}$ & $\begin{array}{c}\text { "Theoritical } \\
\text { Peak" } \\
\text { Mflop/s }\end{array}$ \\
\hline Siemens VP100-EX (7 ns) & Fortran 77/VP V10L30 & 18 & 254 & 428 \\
\hline Sun 670MP Ross Hypersparc(55Mhz) & -cg89 -dalign -libmil -O4 & 18 & & \\
\hline Pentium $133 \mathrm{MHz}$ & $\begin{array}{l}\text { g77 -march=pentium -O3 } \\
\text {-fomit-frame-pointer -funroll-loops }\end{array}$ & 17.61 & 60.65 & 133 \\
\hline Apple PowerMacintosh 8100/100 & Motorola MF77 -O4 & 17 & & \\
\hline Apple Power Mac 6500/275 & Absoft f77 v4.4-O & 17 & & \\
\hline Alliant FX/2800-200 (4 proc) & fortran 1.1.27 -O -inline & 17 & 94 & 160 \\
\hline Amdahl 1100 & 77/VP V10L20 & 17 & 248 & 285 \\
\hline CDC CYBER 205 (4-pipe) & FTN & 17 & 195 & 400 \\
\hline CDC CYBER 205 (2-pipe) & FTN & 17 & 113 & 200 \\
\hline Convex C-3210 (1 proc.) & fc $-\mathrm{O} 2$-uo $-\mathrm{pp}=\mathrm{fcpp} 1$-is . & 17 & 44 & 50 \\
\hline Convex C-210 (1 proc.) & $6.1-\mathrm{O} 2-\mathrm{uo}-\mathrm{pp}=\mathrm{fcpp} 1-\mathrm{is}$ & 17 & 44 & 50 \\
\hline Cray XMS (55 ns) & cf77 5.0 -Zp -Wd-e68 & 17 & 34 & 36 \\
\hline Hitachi S-810/20 & FORT77/HAP & 17 & & 840 \\
\hline IBM ES/9000 Model 210 VF (15 ns) & VAST-2/VS Fortran V2R4 & 17 & 72 & 133 \\
\hline Siemens VP50-EX (7 ns) & Fortran 77/VP V10L30 & 17 & 238 & 285 \\
\hline Multiflow TRACE 14/300 & Fortran 2.2.1 & 17 & 42 & 63 \\
\hline Amdahl 500 & 77/VP V10L20 & 16 & 133 & 142 \\
\hline Fujitsu VP-100 & Fortran 77 & 16 & & 267 \\
\hline Hitachi M680H/vector & Fort 77 E2 V04-0I & 16 & & \\
\hline Hitachi S-810/10 & HAP V21.00 & 16 & & 315 \\
\hline IBM 3090/600J VF (6 proc, 14.5 ns) & & & 540 & 828 \\
\hline IBM 3090/500J VF (5 proc, 14.5 ns) & & & 458 & 690 \\
\hline IBM 3090/400J VF (4 proc, 14.5 ns) & & & 370 & 552 \\
\hline IBM 3090/380J VF (3 proc, 14.5 ns) & & & 282 & 414 \\
\hline IBM 3090/300J VF (3 proc, 14.5 ns) & & & 284 & 414 \\
\hline IBM 3090/280J VF (2 proc, 14.5 ns) & & & 191 & 276 \\
\hline IBM 3090/200J VF (2 proc, 14.5 ns) & & & 192 & 276 \\
\hline IBM 3090/180J VF (1 proc, $14.5 \mathrm{~ns})$ & VS Fortran V2R3 & 16 & 97 & 138 \\
\hline PowerPC $601 / 100 \mathrm{MHz}$ & LS Fortran 1.5 prerelease & 16 & & \\
\hline SGI Crimson(1 proc $50 \mathrm{MHz}$ R4000) & -O2 -mips2 -G 8192 & 16 & 32 & 50 \\
\hline SGI $4 \mathrm{D} / 380(8$ proc $) 33 \mathrm{MHz}$ & $\mathrm{f} 77-\mathrm{O} 2-\mathrm{mp}$ & 16 & 60 & 106 \\
\hline SGI Indigo2 Extreme(R4000/100MHz) & -O2 -mips2 -G 8192 & 15 & & \\
\hline FPS Model 511 & F77 4.2 & 15 & 56 & 67 \\
\hline Hitachi M680H & Fort 77 E2 V04-0I & 15 & & \\
\hline IBM RISC Sys/6000-930 (25 MHz) & v2.2.1 xlf -O -P -Wp,-ea478 & 15 & 42 & 50 \\
\hline IBM RISC Sys/6000-530 (25 MHz) & v2.2.1 xlf -O -P -Wp,-ea478 & 15 & 42 & 50 \\
\hline IBM RISC Sys/6000-340 (33 MHz) & v2.2.1 xlf -O -P -Wp,-ea478 & 15 & 49 & 66 \\
\hline
\end{tabular}




\begin{tabular}{|c|c|c|c|c|}
\hline Computer & $\begin{array}{c}\text { "LINPACK Benchmark" } \\
\text { OS/Compiler }\end{array}$ & $\begin{array}{c}n=100 \\
\text { Mflop/s }\end{array}$ & $\begin{array}{c}\text { "TPP" } \\
\text { Best } \\
\text { Effort } \\
\text { n=1000 } \\
\text { Mflop/s }\end{array}$ & $\begin{array}{c}\text { "Theoritical } \\
\text { Peak" } \\
\text { Mflop/s }\end{array}$ \\
\hline IBM ES/9000-511 (1 proc 11ns) & VAST-2/VS Fortran V2R5 & 15 & & \\
\hline Kendall Square (32 proc) & & & 513 & 1280 \\
\hline Kendall Square (16 proc) & & & 307 & 640 \\
\hline Kendall Square ( 8 proc) & & & 146 & 320 \\
\hline Kendall Square (4 proc) & & & 47 & 160 \\
\hline Kendall Square (1 proc) & ksrf77 -O2 -r8 -inline_auto & 15 & 31 & 40 \\
\hline NAS AS/EX 60 & Fortran & 15 & & 40 \\
\hline SGI 4D/440(4 proc) $40 \mathrm{MHz}$ & $\mathrm{f} 77$-O2 -mp & 15 & 42 & 64 \\
\hline Siemens H120F & Fortran 77 & 15 & & \\
\hline Apple Power Mac 5500/250 & Absoft f77 v4.4-O & 14 & & \\
\hline Power Computing 100/601/100 & Absoft f77 Power PC v4.1 & 14 & & \\
\hline Cydrome CYDRA 5 & Fortran 77 Rel 2.4.1 & 14 & & 25 \\
\hline Fujitsu VP-50 & Fortran 77 & 14 & & 133 \\
\hline IBM ES/9000 Model 190 VF(15 ns) & VAST-2/VS Fortran V2R4 & 14 & 60 & 133 \\
\hline IBM ES/9221-191 (16 ns) & VAST-2/VS Fortran 2.5 & 14 & & \\
\hline Apple Power Mac 7100/80 & Absoft f77 Power PC v4.1 & 13 & & \\
\hline DELL XMT5133 Pentium 133MHz & PS 32 NT V 1.0 /G5 /Oxb2 & 14 & & \\
\hline IBM POWERPC 250 (66 MHz) & -O-Pv-Wp-ea478-g1-qarch=pwrx & 13 & & 66 \\
\hline IBM 3090/180E VF & VS $2.1 .1 \mathrm{opt}=3$ & 13 & 71 & 116 \\
\hline SGI 4D/340(4 proc) $33 \mathrm{MHz}$ & $\mathrm{f} 77-\mathrm{O} 2-\mathrm{mp}$ & 13 & 36 & 53 \\
\hline Apple Power Mac 7500/100 & Absoft f77 Power PC v4.1 & 12 & & \\
\hline Apple Power Mac 8100/80 & Absoft f77 Power PC v4.1 & 12 & & \\
\hline CDC CYBER 990E & FTN V2 VL=HIGH & 12 & & \\
\hline Cray-1S (12.5 ns, 1983 run) & CFT 1.12 & 12 & 110 & 160 \\
\hline Gateway 2000 P5-100XL & MS PS 32 /G5 /Ox /D "NDEBUG" & 12 & & \\
\hline IBM 3090/180 VF & VS Fortran V2 & 12 & 65 & 108 \\
\hline IBM RISC Sys/6000-520H(25 MHz) & v2.2.1 xlf -O -P -Wp,-ea478 & 12 & 37 & 50 \\
\hline IBM RISC Sys/6000-320H(25 MHz) & v2.2.1 xlf -O -P -Wp,-ea478 & 12 & 37 & 50 \\
\hline SGI Indigo $400050 \mathrm{MHz}$ & -O2 -mips2 -G 8192 -sopt & 12 & & \\
\hline Stardent 3040 & 3.0 -inline $-\mathrm{nmax}=300$ & 12 & 77 & 128 \\
\hline Stardent 3030 & 3.0 -inline $-\mathrm{nmax}=300$ & 12 & 63 & 96 \\
\hline Stardent 2040 (Stellar GS2000) & $\mathrm{f} 77$-O3 -is R2.1 & 12 & & 40 \\
\hline Stardent 1040 (Stellar GS1000) & $\mathrm{f} 77$-O3 -is -re R2.0 & 12 & & 40 \\
\hline CDC 4680InfoServer (60 MHz) & f77 2.20 -O3 -mips2 -Wb,-r6000 & 11 & & \\
\hline Cray S-MP/MCP101 (1 proc. 25 ns) & MCP Release 2.2 & 11 & 31 & 40 \\
\hline FPS $510 \mathrm{~S}$ MCP101 (1 proc. $25 \mathrm{~ns})$ & pgf77 -O4 & 11 & 30 & 40 \\
\hline
\end{tabular}




\begin{tabular}{|c|c|c|c|c|}
\hline Computer & $\begin{array}{c}\text { "LINPACK Benchmark" } \\
\text { OS/Compiler }\end{array}$ & $\begin{array}{c}\mathrm{n}=100 \\
\mathrm{Mflop} / \mathrm{s}\end{array}$ & $\begin{array}{c}\text { "TPP" } \\
\text { Best } \\
\text { Effort } \\
\text { n=1000 } \\
\text { Mflop/s }\end{array}$ & $\begin{array}{l}\text { "Theoritical } \\
\text { Peak" } \\
\text { Mflop/s }\end{array}$ \\
\hline IBM ES/9000 Model 340 & VAST-2/VS Fortran V2R4 & 11 & & \\
\hline IBM ES/9000-411 (1 proc 11ns) & VAST-2/VS Fortran V2R5 & 11 & & \\
\hline Meiko Comp. Surface (32 proc) & & & 210 & 1280 \\
\hline Meiko Comp. Surface (16 proc) & & & 187 & 640 \\
\hline Meiko Comp. Surface (8 proc) & & & 147 & 320 \\
\hline Meiko Comp. Surface (4 proc) & & & 98 & 160 \\
\hline Meiko Comp. Surface (2 proc) & -O4 -Mvect=smallvect & & 58 & 80 \\
\hline Meiko Comp. Surface (1 proc) & -Minline $=$ daxpy & 11 & 31 & 40 \\
\hline Gateway 2000 P5-90(90 MHz Pentium) & Windows NT /G5 /Oxb2 & 11 & & \\
\hline SGI Power Series 50MHz R4000 & -O2 -mips2 -G 8192 -sopt & 11 & & \\
\hline Stardent 3020 & 3.0 -inline $-\mathrm{nmax}=300$ & 11 & 46 & 64 \\
\hline Sperry $1100 / 90$ ext w/ISP & UCS level 2 & 11 & & \\
\hline Multiflow TRACE 7/300 & Fortran 2.2.1 & 11 & 22 & 31 \\
\hline Alliant FX/2800-200 (2 proc) & fortran 1.1.27 -O -inline & 10 & 53 & 80 \\
\hline Alliant FX/80 (8 proc.) & -O -DAS -inline & 10 & 69 & 188 \\
\hline IBM 3090/180J & VS Fortran V2R3 & 10 & & \\
\hline Intel Paragon (1 proc) & $\begin{array}{l}-\mathrm{O} 4-\text { Mvect }=\text { smallvect }- \text { Minline }=\text { daxpy } \\
- \text { Knoieee }\end{array}$ & 10 & 34 & 50 \\
\hline MIPS RC6280 (60.0MHz) & f77 $2.20-O$ & 10 & 16 & 24 \\
\hline MIPS RC6260 (60.0MHz) & f77 $2.20-O$ & 10 & 16 & 24 \\
\hline Multiflow TRACE 14/200 & Fortran 1.7 & 10 & & 31 \\
\hline Stardent 3010 & $3.0-$ inline $-\mathrm{nmax}=300$ & 10 & 25 & 32 \\
\hline Stardent 1540 (Ardent Titan-4) & & & 47 & 64 \\
\hline Stardent 1530 (Ardent Titan-3) & & & 37 & 48 \\
\hline Stardent 1520 (Ardent Titan-2) & f77 1.0 -O3 -inline & 10 & 25 & 32 \\
\hline Sun Sparc2000(50 MHz)(16 proc) & & & 333 & 800 \\
\hline Sun Sparc2000(50 MHz)(12 proc) & & & 295 & 600 \\
\hline Sun Sparc2000(50 MHz)(8 proc) & & & 223 & 400 \\
\hline Sun Sparc2000(50 MHz)(1 proc) & & & 28 & 50 \\
\hline Sun Sparc1000(50 MHz)(8 proc) & & & 198 & 400 \\
\hline Sun Sparc1000(50 MHz)(4 proc) & & & 107 & 200 \\
\hline Sun Sparc1000(50 MHz)(2 proc) & & & 53 & 100 \\
\hline Sun Sparc1000(50 MHz)(1 proc) & & & 25 & 50 \\
\hline Sun Sparc10/514(50 MHz)(4 proc) & & & 98 & 200 \\
\hline Sun Sparc10/512(50 MHz)(2 proc) & & & 57 & 100 \\
\hline Sun Sparc10/51(50 MHz)(1 proc) & & & 27 & 50 \\
\hline
\end{tabular}




\begin{tabular}{|c|c|c|c|c|}
\hline Computer & $\begin{array}{c}\text { "LINPACK Benchmark" } \\
\text { OS/Compiler }\end{array}$ & $\begin{array}{c}n=100 \\
\text { Mflop/s }\end{array}$ & $\begin{array}{c}\text { "TPP" } \\
\text { Best } \\
\text { Effort } \\
\text { n=1000 } \\
\text { Mflop/s }\end{array}$ & $\begin{array}{c}\text { "Theoritical } \\
\text { Peak" } \\
\text { Mflop/s }\end{array}$ \\
\hline Sun Sparc10/402(40 MHz)(2 proc) & & & 41 & 81 \\
\hline Sun Sparc10/40(40 MHz)(1 proc) & -fast -O4 -unroll=4 -Bstatic & 10 & 23 & 40 \\
\hline Intel iPSC/Delta (512 proc) & & & 446 & 20480 \\
\hline Intel iPSC/Delta (256 proc) & & & 418 & 10240 \\
\hline Intel iPSC/Delta (128 proc) & & & 393 & 5120 \\
\hline Intel iPSC/Delta (64 proc) & & & 352 & 2560 \\
\hline Intel iPSC/Delta (32 proc) & & & 304 & 1280 \\
\hline Intel iPSC/Delta (16 proc) & & & 231 & 640 \\
\hline Intel iPSC/Delta (8 proc) & & & 163 & 320 \\
\hline Intel iPSC/Delta (4 proc) & & & 100 & 160 \\
\hline Intel iPSC/Delta (2 proc) & if77 -O4 -Mvect=smallvect & & 58 & 80 \\
\hline Intel iPSC/Delta (1 proc) & -Minline=daxpy - Knoieee & 9.8 & 34 & 40 \\
\hline Intel iPSC/860 d7 (128 proc) & & & 219 & 5120 \\
\hline Intel iPSC/860 d6 (64 proc) & & & 208 & 2560 \\
\hline Intel iPSC/860 d5 (32 proc) & & & 167 & 1280 \\
\hline Intel iPSC/860 d4 (16 proc) & & & 131 & 640 \\
\hline Intel iPSC/860 d3 (8 proc) & & & 103 & 320 \\
\hline Intel iPSC/860 d2 (4 proc) & & & 75 & 160 \\
\hline Intel iPSC/860 d1 (2 proc) & if77 -O4 -Mvect=smallvect & & 52 & 80 \\
\hline Intel iPSC/860 d0 (1 proc) & -Minline=daxpy - Knoieee & 9.8 & 34 & 40 \\
\hline SGI $4 \mathrm{D} / 240(4$ proc $) 25 \mathrm{MHz}$ & $\mathrm{f} 77-\mathrm{O} 2-\mathrm{mp}$ & 9.8 & 28 & 40 \\
\hline Apple Power Mac 6100/66 & Absoft f77 Power PC v4.1 & 9.7 & & \\
\hline Apple Power Macintosh 6100/60 & Absoft v4.0 F77 -O & 9.6 & & \\
\hline IBM 3090/180S & VS Fortran 2.3.0 & 9.6 & 92 & 133 \\
\hline Alliant FX/80 (7 proc.) & -O -DAS -inline & 9.5 & 63 & 165 \\
\hline CDC CYBER 4680 & f77 $2.11 .2 \mathrm{o} 2$ & 9.4 & & \\
\hline IBM Power Vis. Sys. (32 proc.) & & & 310 & 1280 \\
\hline IBM Power Vis. Sys. (1 proc.) & $-\mathrm{O} 4-$ Minline $=$ daxpy & 9.3 & & \\
\hline NAS AS/EX 50 & Fortran & 9.3 & & 28 \\
\hline Sun SPARCsystem 10/30 36MHz & f77 -O4 -cg89 -libmil -native & 9.3 & & \\
\hline SGI 4D/420(2 proc) $40 \mathrm{MHz}$ & $\mathrm{f} 77-\mathrm{O} 2-\mathrm{mp}$ & 9.3 & 23 & 32 \\
\hline IBM RISC Sys/6000-520 (20 MHz) & v2.2.1 xlf -O -P -Wp,-ea478 & 9.0 & 29 & 40 \\
\hline IBM RISC Sys/6000-320 (20 MHz) & v2.2.1 xlf -O -P -Wp,-ea478 & 9.0 & 29 & 40 \\
\hline IBM ES/9000-180 VF(15 ns) & VAST-2/VS Fortran V2R4 & 8.9 & 48 & 133 \\
\hline Solbourne 6/904 (Viking sparc) & f77 -O3 -cg89 -dalign & 8.9 & & \\
\hline Intel Pentium $75 \mathrm{MHz}$ & g77 -march=pentium $-\mathrm{O} 3$ & 8.92 & 30.8 & 75 \\
\hline
\end{tabular}




\begin{tabular}{|c|c|c|c|c|}
\hline Computer & $\begin{array}{c}\text { "LINPACK Benchmark" } \\
\text { OS/Compiler }\end{array}$ & $\begin{array}{c}n=100 \\
\text { Mflop/s }\end{array}$ & $\begin{array}{c}\text { "TPP" } \\
\text { Best } \\
\text { Effort } \\
\text { n=1000 } \\
\text { Mflop/s }\end{array}$ & $\begin{array}{l}\text { "Theoritical } \\
\text { Peak" } \\
\text { Mflop/s }\end{array}$ \\
\hline & -fomit-frame-pinter -funroll-loops & & & \\
\hline IBM RISC Sys/6000-230 (45 MHz) & v2.3.0 xlf -O -P -Wp,-ea478 & 8.8 & 19 & 90 \\
\hline DEC VAXvector $6000 / 520$ (2 proc) & Fortran HPO V1.2 & 8.8 & 51 & 90 \\
\hline Comparex 8/92 (Fujitsu M382) & VS/FORTRAN 2.4.0 & 8.7 & & \\
\hline DEC VAXstation 4000-90 & V 5.2 & 8.7 & & \\
\hline Apple Power Macintosh 7100/66 & Absoft v4.0 F77 -O & 8.6 & & \\
\hline IBM ES/9000-311 (1 proc 11ns) & VAST-2/VS Fortran V2R5 & 8.6 & & \\
\hline IBM ES/9000 Model 320 & VAST-2/VS Fortran V2R4 & 8.5 & & \\
\hline NAS AS/9160 & VAST/VS $1.4 .1 \mathrm{opt}=3$ & 8.3 & & \\
\hline Alliant FX/80 (5 proc.) & -O -DAS -inline & 8.1 & 49 & 118 \\
\hline IBM ES/9000 Model 260 & VAST-2/VS Fortran V2R4 & 8.0 & & \\
\hline SCS-40 & CFT 1.13 & 8.0 & 17 & 45 \\
\hline SGI $4 \mathrm{D} / 320(2$ proc $) 33 \mathrm{MHz}$ & $\mathrm{f} 77-\mathrm{O} 2-\mathrm{mp}$ & 8.0 & 20 & 26 \\
\hline IBM ES/9000 Model 210 & VAST-2/VS Fortran V2R4 & 7.7 & & \\
\hline IBM ES/9000 Model 320 & VS/FORTRAN V2R4 & 7.6 & & \\
\hline IBM 3090/120E VF & VS $2.1 .1 \mathrm{opt}=3$ & 7.5 & 54 & 108 \\
\hline IBM 3090/180E & VS $2.1 .1 \mathrm{opt}=3$ & 7.4 & 71 & 116 \\
\hline Siemens $7890 \mathrm{~F}$ & Fortran 77 V10.3 & 7.2 & & \\
\hline Convex C-130 & Fortran 4.0 & 7.2 & 31 & 36 \\
\hline Alliant FX/80 (4 proc.) & -O -DAS -inline & 7.2 & 33 & 94 \\
\hline DEC VAXvector $6000 / 510$ (1 proc) & Fortran HPO V1.2 & 7.0 & 28 & 45 \\
\hline CECpx XL 560 Pentium $60 \mathrm{MHz}$ & $10.5 \mathrm{wfc} 386 / \mathrm{l}=\operatorname{dos} 4 \mathrm{~g} / \mathrm{ox}$ & 7.2 & & \\
\hline Sun SPARCsystem 10/41 40MHz & f77 -native -fast -O4 -Bstatic & 7.0 & & \\
\hline Stardent 1510 (Ardent Titan-1) & f77 1.0 -O2 -inline & 6.9 & 13 & 16 \\
\hline IBM 3090/180 & VS opt $=3$ & 6.8 & 65 & 108 \\
\hline Alliant FX/40 (4 proc.) & -O -DAS -inline & 6.7 & 33 & 94 \\
\hline IBM RS/6000-N40(PowerPC601 50MHz) & xlf -O -P -Wp,-ea478 & 6.7 & & 50 \\
\hline IBM RISC Sys/6000-M20 (33 MHz) & v2.3.0 xlf -O -P -Wp,-ea478 & 6.6 & 14 & 66 \\
\hline IBM RISC Sys/6000-M2A (33 MHz) & v2.3.0 xlf -O -P -Wp,-ea478 & 6.6 & 14 & 66 \\
\hline IBM ES/9000 Model 190 & VAST-2/VS Fortran V2R4 & 6.6 & & 133 \\
\hline Convex C-120 & fe 5.1 & 6.5 & 17 & 20 \\
\hline IBM RISC Sys/6000-220 (33 MHz) & v2.2.1 xlf -O -P -Wp,ea478 & 6.5 & 14 & 66 \\
\hline Alliant FX/4 (4 proc.) & -O -DAS -inline & 6.4 & 21 & 47 \\
\hline Alliant FX/2800-200 (1 proc) & fortran 1.1.27 -O -inline & 6.4 & 28 & 40 \\
\hline Apple PowerBook PB1400cs(133 MHz) & MF -O4 -Asched $=2, \operatorname{targ}=604$ & 6.3 & & \\
\hline Fujitsu M-380 & Fortran $77, \mathrm{opt}=3$ & 6.3 & & \\
\hline DEC VAX 6620 & V5.5 & 6.2 & & \\
\hline Multiflow TRACE 7/200 & Fortran 1.4 & 6.0 & & 15 \\
\hline
\end{tabular}




\begin{tabular}{|c|c|c|c|c|}
\hline Computer & $\begin{array}{c}\text { "LINPACK Benchmark" } \\
\text { OS/Compiler }\end{array}$ & $\begin{array}{c}n=100 \\
\text { Mflop/s }\end{array}$ & $\begin{array}{c}\text { “TPP" } \\
\text { Best } \\
\text { Effort } \\
\text { n=1000 } \\
\text { Mflop/s }\end{array}$ & $\begin{array}{l}\text { "Theoritical } \\
\text { Peak" } \\
\text { Mflop/s }\end{array}$ \\
\hline SGI 4D/420(1 proc) $40 \mathrm{MHz}$ & f77 -O2 & 6.0 & 12 & 16 \\
\hline Apple Performa $6230 \mathrm{CD} / 603 / 75$ & Absoft f77 Power PC v4.1 & 5.9 & & \\
\hline Siemens $7890 \mathrm{G}$ & Fortran $77 \mathrm{~V} 10.3 \mathrm{opt}=4$ & 5.9 & & \\
\hline IBM 3090/150E & VS $2.1 .1 \mathrm{opt}=3$ & 5.9 & 64 & 112 \\
\hline FPS-264 (M64/60) & F02 APFTN64 OPT=4 & 5.9 & 34 & 38 \\
\hline Alliant FX/80 (3 proc.) & -O -DAS -inline & 5.9 & 32 & 71 \\
\hline SGI $4 \mathrm{D} / 220(2$ proc $) 25 \mathrm{MHz}$ & $\mathrm{f} 77-\mathrm{O} 2-\mathrm{mp}$ & 5.9 & 15 & 20 \\
\hline Apollo DN10000 & $\mathrm{f} 77,10.7$ & 5.8 & & \\
\hline DEC VAX 4000 & opt=4, DEC Fortran V6.5 & 5.7 & & \\
\hline HP9000/J200 (100 MHz) & fort77 -o & 5.5 & & \\
\hline Alliant FX/40 (3 proc.) & -O -DAS -inline & 5.6 & 27 & 71 \\
\hline Gateway P5-60 (60 MHz Pentium) & F77L-EM32 5.01 /4 /Z1 & 5.4 & & \\
\hline DEC 5900 RISC & Ultrix 4.1 & 5.3 & & \\
\hline DEC 5000/240 & Ultrix & 5.3 & & \\
\hline Gateway P5-60 (60 MHz Pentium) & 77/32/mf/d1/warn/5/fp5/ot & 5.3 & & \\
\hline Alliant FX/4 (3 proc.) & -O -DAS -inline & 5.1 & 17 & 35 \\
\hline CDC 4330-300 (33 MHz) & f77 $2.20-\mathrm{O} 3$ & 5.1 & & \\
\hline Number-Smasher $86040 \mathrm{MHz}$ & NDP -vast-inline-on-OLM-fdiv & 5.1 & & \\
\hline VAXstation 4000-90 & DEC FORTRAN V5.2 & 5.1 & & \\
\hline DEC VAX 6000/610 (1 proc) & VMS V5.2 & 5.0 & & \\
\hline Intel iPSC/2 d4/VX (16 proc) & & & 39 & \\
\hline Intel iPSC/2 d5/VX (32 proc) & & & 52 & \\
\hline SGI $4 \mathrm{D} / 310(1$ proc $) 33 \mathrm{MHz}$ & $\mathrm{f} 77-\mathrm{O} 2$ & 5.0 & 10 & 13 \\
\hline Honeywell DPS90 & ES F77V 1.0 & 5.0 & & \\
\hline Siemens 7890D & Fortran $77 \mathrm{~V} 10.3$ & 5.0 & & \\
\hline IBM ES/9000 Model 180 (15 ns) & VAST-2/VS Fortran V2R4 & 4.9 & & \\
\hline CDC CYBER 875 & FTN 5 opt $=3$ & 4.8 & & \\
\hline Number Smasher i860 40MHz & -on -OLM -fdiv -inline & 4.7 & & 40 \\
\hline CDC CYBER 176 & FTN $5.1 \mathrm{opt}=2$ & 4.6 & & \\
\hline MIPS RC3360 (33.3MHz) & f77 $2.20-\mathrm{O}$ & 4.5 & 11 & 13 \\
\hline Alliant FX/80 (2 proc.) & -O -DAS -inline & 4.4 & 22 & 47 \\
\hline AMD 486DX5-133 & f2c and $g c c 2.7 .0$ & 4.4 & & \\
\hline Alliant FX/40 (2 proc.) & -O -DAS -inline & 4.3 & 19 & 47 \\
\hline NAS AS/EX 30 & VS $1.4 .1 \mathrm{opt}=3$ & 4.3 & & \\
\hline SGI 4D/35 & $\mathrm{f} 77-\mathrm{O} 3$ & 4.3 & & \\
\hline Sun 4/600 MP & f77 1.4 -O3 -cg89 -dalign & 4.3 & & \\
\hline
\end{tabular}




\begin{tabular}{|c|c|c|c|c|}
\hline Computer & $\begin{array}{c}\text { "LINPACK Benchmark" } \\
\text { OS/Compiler }\end{array}$ & $\begin{array}{c}n=100 \\
\text { Mflop/s }\end{array}$ & $\begin{array}{c}\text { "TPP" } \\
\text { Best } \\
\text { Effort } \\
\text { n=1000 } \\
\text { Mflop/s }\end{array}$ & $\begin{array}{c}\text { "Theoritical } \\
\text { Peak" } \\
\text { Mflop/s }\end{array}$ \\
\hline IBM ES/9221-170 (16 ns) & VAST-2/VS Fortran 2.5 & 4.1 & & \\
\hline Sun SPARCstation IPX & f77 1.4 -O3 -cg89 -dalign & 4.1 & & \\
\hline Sun 4/50 IPX & f77 1.4 -O3 -cg89 -dalign & 4.1 & & \\
\hline CDC CYBER 4360 & f77 $2.11 .2 \mathrm{o} 2$ & 4.0 & & \\
\hline Sun SPARCstation 2 & f77 1.4 -O3 -cg89 -dalign & 4.0 & & \\
\hline SGI Indigo 33MHz R3000 & -O2 -G 8192 -sopt & 4.0 & & \\
\hline Amdahl 5860 HSFPF & H enhanced opt $=3$ & 3.9 & & \\
\hline MIPS M/2000 (25.0MHz) & f77 $2.20-\mathrm{O}$ & 3.9 & 7.9 & 10 \\
\hline MIPS RC3260 (25.0MHz) & f77 $2.20-\mathrm{O}$ & 3.9 & 7.9 & 10 \\
\hline Alliant FX/4 (2 proc.) & -O -DAS -inline & 3.8 & 12 & 24 \\
\hline SGI $4 \mathrm{D} / 210(1$ proc $) 25 \mathrm{MHz}$ & $\mathrm{f} 77-\mathrm{O} 2$ & 3.9 & 7.8 & 10 \\
\hline Amdahl 5860 HSFPF & VS opt $=3$ & 3.8 & & \\
\hline CDC 4320 & f77 $2.20 \mathrm{opt}=02$ & 3.7 & & \\
\hline DEC station 5000/200 (25 Mhz) & MIPS f77 2.0 & 3.7 & & \\
\hline MIPS RS3230 (25.0MHz) & f77 $2.20-\mathrm{O}$ & 3.7 & 7.8 & 10 \\
\hline DEC VAXvector 6000/420 (2 proc) & Fortran HPO V1.0 & & 43 & 90 \\
\hline DEC VAXvector $6000 / 410$ (1 proc) & Fortran HPO V1.0 & 3.6 & 24 & 45 \\
\hline Sun $4 / 490$ & $4.1 .1 \mathrm{f} 77-\mathrm{O} 3$ & 3.6 & & \\
\hline CDC 4330 & $\mathrm{f} 772.20 \mathrm{opt}=02$ & 3.5 & & \\
\hline Apple Power Macintosh 6100/60 & Absoft F77 SDK & 3.4 & & \\
\hline NAS 8093 w/HSA & VS $1.4 .0 \mathrm{opt}=3$ & 3.5 & & \\
\hline CDC 7600 & FTN & 3.3 & & \\
\hline Sun Sparc ELC & -dalign -xcg89 -fsimple -O4 & 3.3 & & \\
\hline CDC CYBER 960-31 & NOS/VE 1.3.1 FTN 1.6 & 3.1 & & \\
\hline Gould NP1 & Fortran & 3.1 & & \\
\hline IBM 3090/120E & VS $2.1 .1 \mathrm{opt}=3$ & 3.1 & 54 & 108 \\
\hline MIPS RC3240 (25.0MHz) & f77 $2.20-\mathrm{O}$ & 3.1 & 7.1 & 10 \\
\hline Tadpole SPARCbook 2 & $\mathrm{f} 77-\mathrm{O}$ & 3.1 & & \\
\hline CDC CYBER 4340 & f77 $2.11 .2 \mathrm{o} 2$ & 3.0 & & \\
\hline Convex $\mathrm{C}-1 / \mathrm{XP}$ & Fortran 2.0 & 3.0 & & 20 \\
\hline DEC VAX 6540 & VMS 5.4-2 & 3.0 & & \\
\hline FPS-264/20 (M64/50) & F02 APFTN64 OPT=4 & 3.0 & 17 & \\
\hline Harris Nighthawk 4802 (88100) & f77 & 3.0 & & \\
\hline Convex $\mathrm{C}-1 / \mathrm{XL}$ & Fortran 1.6 & 2.9 & & 20 \\
\hline IBM ES/9000 Model 150 & VS Fortran V2R4 & 2.9 & & \\
\hline
\end{tabular}




\begin{tabular}{|c|c|c|c|c|}
\hline Computer & $\begin{array}{c}\text { "LINPACK Benchmark" } \\
\text { OS/Compiler }\end{array}$ & $\begin{array}{c}\mathrm{n}=100 \\
\mathrm{Mflop} / \mathrm{s}\end{array}$ & $\begin{array}{c}\text { “TPP" } \\
\text { Best } \\
\text { Effort } \\
\text { n=1000 } \\
\text { Mflop/s }\end{array}$ & $\begin{array}{c}\text { "Theoritical } \\
\text { Peak" } \\
\text { Mflop/s }\end{array}$ \\
\hline NAS AS/EX 25 & VS $1.4 .1 \mathrm{opt}=3$ & 2.9 & & \\
\hline Solbourne $5 / 602$ & f77 (Sun) 1.2 -O3 -dalign & 2.9 & & \\
\hline Sun $4 / 330$ & f77 1.4 -O3 -dalign & 2.7 & & \\
\hline Sun $4 / 370$ & f77 1.3.1 -O3 -cg89 -dalign & 2.7 & & \\
\hline CDC CYBER 760 & FTN 5, opt $=3$ & 2.6 & & \\
\hline CyberPlus & CPFTN 1.1-07 & 2.6 & & \\
\hline IBM 370/195 & H enhanced opt $=3$ & 2.5 & & \\
\hline Sun 4/330 SparcServer & f77 1.2, -O3 -dalign & 2.5 & & \\
\hline Alliant FX/80 (1 proc.) & -O -DAS -inline & 2.4 & 12 & 24 \\
\hline Alliant FX/40 (1 proc.) & -O -DAS -inline & 2.4 & 10 & 24 \\
\hline Gateway 200066 MHz 80486-DX2 & F77L-EM32 5.01 /4 /Z1 & 2.4 & & \\
\hline Apple Mac Quadra 840AV & Absoft -w -v -O -f -s -N40 & 2.3 & & \\
\hline HP-APOLLO 9000/425e (68040) & f77 -O4 rev 10.3.5 & 2.3 & & \\
\hline NAS AS/EX 20 & VS $1.4 .1 \mathrm{opt}=3$ & 2.2 & & \\
\hline Fujitsu AP1000 (512 proc.) & & & 610 & 2844 \\
\hline Fujitsu AP1000 (256 proc.) & & & 333 & 1422 \\
\hline Fujitsu AP1000 (128 proc.) & & & 193 & 711 \\
\hline Fujitsu AP1000 (64 proc.) & & & 100 & 356 \\
\hline Fujitsu AP1000 (1 proc.) & Sun f77 1.3.1 -O3 -dalign & 2.2 & 1.7 & 5.6 \\
\hline HP-APOLLO 9000/425t (68040) & f77 -O4 rev 10.3.4 & 2.2 & & \\
\hline Alliant FX/4 (1 proc.) & -O -DAS -inline & 2.1 & 6.3 & 12 \\
\hline CDC CYBER 175 & FTN 5 opt $=2$ & 2.1 & & \\
\hline CDC CYBER 180-860 & NOS/VE OPT=HIGH & 2.1 & & \\
\hline FPS-M64/30 & APFTN464 OPT $=4$ & 2.1 & 10 & \\
\hline IBM ES/9000 Model 130 & VS Fortran V2R4 & 2.1 & & \\
\hline IBM 3081 K (1 proc.) & H enhanced opt $=3$ & 2.1 & & \\
\hline MIPS M120-5 & UMIPS v.3 3.0 f771.31 -O & 2.1 & 3.6 & 8.3 \\
\hline MIPS M/120 (16.7MHz) & f77 $2.20-\mathrm{O}$ & 2.1 & 4.8 & 6.7 \\
\hline Prism" 486-50 (50 MHz) & Salford v2.69 /optimise & 2.1 & & \\
\hline Tadpole SPARCbook (25 MHz) & $\mathrm{f} 77-\mathrm{O}$ & 2.1 & & \\
\hline Apple Macintosh QUADRA 950 & Absoft -w -v -O -f -s -N40 & 2.0 & & \\
\hline CDC 7600 & Local & 2.0 & & \\
\hline IBM 3081 K (1 proc.) & VS opt=3 & 2.0 & & \\
\hline Culler PSC & CSD Fortran 3.21 & 2.0 & & 5 \\
\hline FPS M64/35 & APFTN464 & 2.0 & & \\
\hline
\end{tabular}




\begin{tabular}{|c|c|c|c|c|}
\hline Computer & $\begin{array}{c}\text { "LINPACK Benchmark" } \\
\text { OS/Compiler }\end{array}$ & $\begin{array}{c}n=100 \\
\text { Mflop/s }\end{array}$ & $\begin{array}{c}\text { “TPP" } \\
\text { Best } \\
\text { Effort } \\
\text { n=1000 } \\
\text { Mflop/s }\end{array}$ & $\begin{array}{l}\text { "Theoritical } \\
\text { Peak" } \\
\text { Mflop/s }\end{array}$ \\
\hline Micronics $486-50 \mathrm{MHz}$ EISA2 & NDP Fortran 486: -on & 2.0 & & \\
\hline HP 425T (68040) & & 1.9 & & \\
\hline CDC CYBER 175 & FTN 5 opt $=1$ & 1.8 & & \\
\hline HP 9000 Series 835 & $2.1 \mathrm{fc}-\mathrm{O}$ & 1.8 & & \\
\hline Sperry $1100 / 90$ & FTN opt $=$ ZEO & 1.8 & & \\
\hline Sun SPARCstation 1+ & f77 1.4 -O3 -cg89 -dalign & 1.8 & & \\
\hline ELXSI 6420 (5 proc.) & & & 6.4 & \\
\hline ELXSI 6420 (3 proc.) & & & 4.0 & \\
\hline ELXSI 6420 (2 proc.) & & & 2.7 & \\
\hline ELXSI 6420 (1 proc.) & EMBOS $6.3+\mathrm{opt}+$ inline+vector & 1.7 & 1.4 & \\
\hline FPS-164/364 (M64/40) & F02 APFTN64 OPT=4 & 1.7 & 9 & \\
\hline Honeywell DPS 8/88 & FR7X & 1.7 & & \\
\hline IBM 3033 & $\mathrm{H}$ enhanced opt $=3$ & 1.7 & & \\
\hline IBM 3033 & VS opt $=3$ & 1.7 & & \\
\hline IBM $3081 \mathrm{D}$ & VS opt $=3$ & 1.7 & & \\
\hline MIPS RS2030 (16.7MHz) & f77 $2.20-\mathrm{O}$ & 1.7 & 4.7 & 6.7 \\
\hline Sperry $1100 / 90$ ext & UFTN & 1.7 & & \\
\hline HP 9000 Series $850 \mathrm{w} / \mathrm{fp}$ & $2.0 \mathrm{fc}-\mathrm{O}$ & 1.6 & & \\
\hline Amdahl $470 \mathrm{~V} / 8$ & H enhanced opt $=3$ & 1.6 & & \\
\hline CDC CYBER 170-750 & FTN 5.1, opt=3 & 1.6 & & \\
\hline CDC CYBER 180-850 & NOS/VE OPT $=\mathrm{HIGH}$ & 1.6 & & \\
\hline DECstation 3100 & V3.0/V1.31-O & 1.6 & & \\
\hline DEC 5400 & $\mathrm{f} 77-\mathrm{O} 3$ & 1.6 & & \\
\hline Amdahl $470 \mathrm{~V} / 8$ & VS opt $=3$ & 1.5 & & \\
\hline DEC VAXstation 4000-60 & V 5.2 & 1.5 & & \\
\hline MIPS M/1000 (15.0MHz) & f77 $2.20-\mathrm{O}$ & 1.5 & 3.7 & 6 \\
\hline NAS 8093 & VS $1.4 .0 \mathrm{opt}=3$ & 1.5 & & \\
\hline Siemens $7570-\mathrm{P}$ & For1 1.6A & 1.5 & & \\
\hline ALR $486 / 33$ m-board, $256 \mathrm{~K}$ cache & Lahey F77L3, v5.0 /Z1 & 1.4 & & \\
\hline Apple Mac Quadra 700 & Absoft -w -v -O -f -s -N40 & 1.4 & & \\
\hline Compaq Deskpro 486/331-120 w/487 & Microway NDPF487 -O -OL -on & 1.4 & & \\
\hline NeXTCube & $2.0 \mathrm{gcc} 1.36-\mathrm{O}$ & 1.4 & & \\
\hline Sun SPARCstation 1 & f77 1.3.1 -O3 -cg89 -dalign & 1.4 & & \\
\hline IBM 4381-23 & VS Fortran $2.1 .1 \mathrm{opt}=3$ & 1.3 & & \\
\hline
\end{tabular}




\begin{tabular}{|c|c|c|c|c|}
\hline Computer & $\begin{array}{c}\text { "LINPACK Benchmark" } \\
\text { OS/Compiler }\end{array}$ & $\begin{array}{c}\mathrm{n}=100 \\
\mathrm{Mflop} / \mathrm{s}\end{array}$ & $\begin{array}{c}\text { “TPP” } \\
\text { Best } \\
\text { Effort } \\
\text { n=1000 } \\
\text { Mflop/s }\end{array}$ & $\begin{array}{l}\text { "Theoritical } \\
\text { Peak" } \\
\text { Mflop/s }\end{array}$ \\
\hline Compaq Deskpro 486/331-120 w/487 & Salford FTN77/ optimized & 1.3 & & \\
\hline Compaq Deskpro 486/331-120 w/487 & Watcom WFC386P /OL /OT & 1.3 & & \\
\hline ALR 486/33 m-board, 256K cache & Lahey F77L3, v5.0 /nZ1 & 1.2 & & \\
\hline CDC 7600 & CHAT, No opt & 1.2 & & \\
\hline CSPI MAP-6430 & Fortran 1.5.35 & 1.2 & & \\
\hline DEC VAX 6000/460 (6 proc) & & & 8.4 & 15 \\
\hline DEC VAX $6000 / 450$ (5 proc) & & & 7.1 & 13 \\
\hline DEC VAX $6000 / 440$ (4 proc) & & & 5.8 & 10 \\
\hline DEC VAX 6000/430 (3 proc) & & & 4.4 & 7.6 \\
\hline DEC VAX 6000/420 (2 proc) & & & 3.0 & 5.1 \\
\hline DEC VAX 6000/410 (1 proc) & VMS V5.2 & 1.2 & 1.5 & 2.6 \\
\hline ELXSI 6420 & Fortran $5.14 \mathrm{opt}=10$ & 1.2 & 1.4 & \\
\hline Gateway 2000/Micronics 486DX/33 & $\mathrm{f} 2 \mathrm{c}$ emx/gcc $-\mathrm{O} 2-\mathrm{m} 486$ & 1.2 & & \\
\hline Gateway Pentium $(66 \mathrm{HHz})$ & Lahey F77, 4.00 & 1.2 & & \\
\hline IBM ES/9000 Model 120 & VS Fortran V2R4 & 1.2 & & \\
\hline IBM 370/168 Fast Mult & H Ext & 1.2 & & \\
\hline IBM $438190 \mathrm{E}$ & VS Fortran $2.1 .1 \mathrm{opt}=3$ & 1.2 & & \\
\hline IBM 4381-13 & VS $1.4 .0 \mathrm{opt}=3$ & 1.2 & & \\
\hline MIPS M/800 (12.5MHz) & f77 $1.31-O$ & 1.2 & & 5 \\
\hline Prime P6350 & f77 rev 20.2.b2 -opt & 1.2 & & \\
\hline Siemans 7580-E & BS2000 & 1.2 & & \\
\hline Amdahl $470 \mathrm{~V} / 6$ & $\mathrm{H} \mathrm{opt}=2$ & 1.1 & & \\
\hline Compaq Deskpro 486/331-120 w/487 & Lahey F77L3 /Z1 & 1.1 & & \\
\hline Sun $4 / 260$ & f77 -O sys4-beta2 & 1.1 & 1.1 & 3.3 \\
\hline ES1066 (1 proc. 80 ns Russian) & f77(like IBM VS1.4.1 OPT=3) & 1.0 & & \\
\hline Sony Playstation 2 & gec 2.95.2 Linux & .995 & & \\
\hline CDC CYBER 180-840 & NOS/VE OPT $=\mathrm{HIGH}$ & .99 & & \\
\hline DEC VAX 8800 (4 proc) & & & 4.9 & \\
\hline DEC VAX 8800 (3 proc) & & & 3.7 & \\
\hline DEC VAX 8800 (2 proc) & & & 2.5 & \\
\hline DEC VAX 8550/8700/8800 & VMS v4.5 & .99 & 1.3 & \\
\hline Solbourne & $\mathrm{f} 77-\mathrm{O}$ & .98 & & \\
\hline IBM 4381-22 & VS Fortran $2.1 .1 \mathrm{opt}=3$ & .97 & & \\
\hline IBM 4381 MG2 & VS Fortran opt=3 & .96 & & \\
\hline
\end{tabular}




\begin{tabular}{|c|c|c|c|c|}
\hline Computer & $\begin{array}{c}\text { "LINPACK Benchmark" } \\
\text { OS/Compiler }\end{array}$ & $\begin{array}{c}\mathrm{n}=100 \\
\mathrm{Mflop} / \mathrm{s}\end{array}$ & $\begin{array}{c}\text { “TPP” } \\
\text { Best } \\
\text { Effort } \\
\text { n=1000 } \\
\text { Mflop/s }\end{array}$ & $\begin{array}{c}\text { "Theoritical } \\
\text { Peak" } \\
\text { Mflop/s }\end{array}$ \\
\hline IBM 4381-12 & VS Fortran $1.4 .0 \mathrm{opt}=3$ & .95 & & \\
\hline ICL $3980 \mathrm{w} / \mathrm{FPU}$ & FORTRAN77 PLUS V10.02 & .93 & & \\
\hline IBM-486 33MHz & Microsoft 5.1 & .94 & & \\
\hline Siemens $7860 \mathrm{E}$ & Fortran 77 V10.3 & .92 & & \\
\hline Concurrent $3280 \mathrm{XP}$ & Fortran VII,Z 8.1 & .87 & & \\
\hline MIPS M800 w/R2010 FP & f77 1.10 & .87 & & \\
\hline Gould PN 9005 & VTX/32 2.0 Fortran 77 & .87 & & \\
\hline VAXstation 3100-76 & DEC FORTRAN V5.2 & .85 & & \\
\hline IBM 9370-90 & VS Fortran $1.3 .0 \mathrm{opt}=3$ & .78 & & \\
\hline nCUBE 2,1024 proc & & & 258 & 2409 \\
\hline nCUBE 2, 512 proc & & & 204 & 1205 \\
\hline nCUBE 2, 256 proc & & & 165 & 602 \\
\hline nCUBE 2,128 proc & & & 116 & 301 \\
\hline nCUBE 2,64 proc & & & 76.9 & 151 \\
\hline nCUBE 2,32 proc & & & 46.0 & 75 \\
\hline nCUBE 2,16 proc & & & 26.1 & 38 \\
\hline nCUBE 2,8 proc & & & 14.2 & 19 \\
\hline nCUBE 2, 4 proc & & & 7.50 & 9.4 \\
\hline nCUBE 2, 2 proc & & & 3.91 & 4.7 \\
\hline nCUBE 2,1 proc & Fort $77 / \mathrm{ncc}-\mathrm{O} 3$ & .78 & 2.02 & 2.35 \\
\hline IBM 370/165 Fast Mult & H Ext & .77 & & \\
\hline Prime P9955II & f77 rev 20.2.b2 -opt & .72 & & \\
\hline DEC VAX 8530 & VMS v4.6 & .73 & & \\
\hline HP 9000 Series 850 & $2.0 \mathrm{fc}-\mathrm{O}$ & .71 & & \\
\hline DEC VAX 8650 & VMS v4.5 & .70 & & \\
\hline DEC VAX 8500 & VMS v4.5 & .65 & & \\
\hline HP/Apollo DN4500 (68030 + FPA) & & .60 & & \\
\hline Mentor Graphics Computer & fortran & .60 & & \\
\hline MIPS M/500 ( $8.3 \mathrm{HHz})$ & f77 $1.21-\mathrm{O}$ & .60 & & 3.3 \\
\hline Data General MV/20000 & $\mathrm{f} 77$ & .59 & & \\
\hline IBM 9377-80 & VS Fortran $2.1 .1 \mathrm{opt}=3$ & .58 & & \\
\hline Sperry $1100 / 80 \mathrm{w} / \mathrm{SAM}$ & FTN opt=ZEO & .58 & & \\
\hline CDC CYBER 930-31 & NOS/VE 1.2 .2 & .58 & & \\
\hline Russian PS-2100 & FORTRAN-PS & .57 & 1.6 & \\
\hline
\end{tabular}




\begin{tabular}{|c|c|c|c|c|}
\hline Computer & $\begin{array}{c}\text { "LINPACK Benchmark" } \\
\text { OS/Compiler }\end{array}$ & $\begin{array}{c}n=100 \\
\text { Mflop/s }\end{array}$ & $\begin{array}{c}\text { “TPP" } \\
\text { Best } \\
\text { Effort } \\
\text { n=1000 } \\
\text { Mflop/s }\end{array}$ & $\begin{array}{c}\text { "Theoritical } \\
\text { Peak" } \\
\text { Mflop/s }\end{array}$ \\
\hline Gateway 486DX-2 (66HHz) & Lahey F77, 4.00 & .56 & & \\
\hline Harris H1200 & VOS 4.1 opt g & .56 & & \\
\hline HP/Apollo DN4500 (68030) & & .55 & & \\
\hline HP 9000 Series 825 & $2.0 \mathrm{fc}-\mathrm{O}$ & .53 & & \\
\hline HP-APOLLO 9000/400t (68030) & f77 -O4 rev 10.8(190) & .51 & & \\
\hline Harris HCX-9 & hf77 -O3 & .50 & & \\
\hline Pyramid 9810 & OSx 4.0 & .50 & & \\
\hline HP 9000 Series 840 & $2.0 \mathrm{fc}-\mathrm{O}$ & .49 & & \\
\hline DEC VAX 8600 & VMS v4.5 & .48 & & \\
\hline Harris HCX-7 w/fpp & f77 1.0 & .48 & & \\
\hline CDC 6600 & FTN $4.6 \mathrm{opt}=2$ & .48 & & \\
\hline CDC CYBER 170-835 & FTN 5 opt=2 & .47 & & \\
\hline CCI Power 6/32 w/fpa & UNIX 4.2 bsd f77 & .47 & & \\
\hline IBM 4381-21 & VS Fortran $2.1 .1 \mathrm{opt}=3$ & .47 & & \\
\hline Sperry 7000 & 4.2 & .47 & & \\
\hline Gould PN9000 & UNIX & .47 & & \\
\hline SUN-3/260 + FPA & 3.2 f77 -O -ffpa & .46 & & \\
\hline IBM 4381 MG1 & VS Fortran opt $=3$ & .46 & & \\
\hline DEC VAX 6210 (1 proc.) & VMS v5.0 & .46 & & \\
\hline CDC CYBER 170-835 & FTN 5 opt=1 & .44 & & \\
\hline HP 9000 Series 840 & HP-UX 14.3 & .43 & & \\
\hline IBM RT 135 & AIX-2.2 & .42 & & \\
\hline Harris H1000 & VOS 3.3 opt g & .41 & & \\
\hline microVAX 3200/3500/3600 & VMS v4.6 & .41 & & \\
\hline Apple Macintosh IIfx & A/UX $2.0 \mathrm{f} 77$ & .41 & & \\
\hline Apollo DN5xxT FPX & DOMAIN/IX SR9.7 opt 4 & .40 & & \\
\hline microVAX 3200/3500/3600 & ULTRIX 2.2/VFU & .40 & & \\
\hline IBM 9370-60 & VS Fortran $1.4 .0 \mathrm{opt}=3$ & .40 & & \\
\hline Sun-3/160 + FPA & $3.2 \mathrm{f} 77-\mathrm{O}-\mathrm{ffpa}$ & .40 & & \\
\hline Prime P9755 & f77 rev 20.2.b2 -opt & .40 & & \\
\hline Ridge 3200 Model 90 & ROS/rf & .39 & & \\
\hline IBM 4381-11 & VS Fortran $1.4 .0 \mathrm{opt}=3$ & .39 & & \\
\hline Gould 32/9705 mult acc & fort77+ 4.3 & .39 & & \\
\hline NORSK DATA ND-570/2 & Fortran-500-E & .38 & & \\
\hline
\end{tabular}




\begin{tabular}{|c|c|c|c|c|}
\hline Computer & $\begin{array}{c}\text { "LINPACK Benchmark" } \\
\text { OS/Compiler }\end{array}$ & $\begin{array}{c}n=100 \\
\text { Mflop/s }\end{array}$ & $\begin{array}{c}\text { "TPP" } \\
\text { Best } \\
\text { Effort } \\
\text { n=1000 } \\
\text { Mflop/s }\end{array}$ & $\begin{array}{l}\text { "Theoritical } \\
\text { Peak" } \\
\text { Mflop/s }\end{array}$ \\
\hline Sperry $1100 / 80$ & FTN opt=ZEO & .38 & & \\
\hline Apple Mac IIfx & Absoft -w -v -O -f -s & .37 & & \\
\hline CDC CYBER 930-11 & NOS/VE OPT=High & .37 & & \\
\hline CSA w/T800C-20 & Fortran 3L & .37 & & \\
\hline Inmos T800 (20 MHz) & Fortran 3L -:o0 & .37 & & \\
\hline Sequent Symmetry (386 w/fpa) & Fortran -fpa -O3 & .37 & & \\
\hline CONCEPT 32/8750 & UTX/32 & .36 & & \\
\hline Celerity C 1230 & UNIX 4.2 bsd f77 & .36 & & \\
\hline IBM RT PC 6150/115 fpa2 & f77 & .36 & & \\
\hline IBM 9373-30 & VS Fortran $2.1 .1 \mathrm{opt}=3$ & .36 & & \\
\hline CDC 6600 & RUN & .36 & & \\
\hline Gould PN9080 & UTX/32 & .35 & & \\
\hline Prime 9950 & F77 19.4.2 & .34 & & \\
\hline Opus Series 300pm $30 \mathrm{MHz}$ & UNIX Greenhills & .33 & & \\
\hline Masscomp MC5600 w/fpa & $\mathrm{f} 77 \mathrm{v} 1.2-\mathrm{O} 3 \mathrm{rtv}$ v3.1 & .33 & & \\
\hline Data General MV/10000 & f77 opt level 2 & .30 & & \\
\hline IBM 4361 MG5 & VS Fortran opt $=3$ & .30 & & \\
\hline DATEK 80386-33 /w 64KB Cache & MS Fortran 5.0 -Ox -AH -G2 & .27 & & \\
\hline Inmos T800 (20 MHz) & Fortran 3L -:o1 & .26 & & \\
\hline Apollo DN3500 & FTN -CPU 3000 -opt 4 & .25 & & \\
\hline IRIS 2400 Turbo/FPA & $\mathrm{f} 77$ & .24 & & \\
\hline CDC CYBER 180-830 & NOS/VE OPT=HIGH & .24 & & \\
\hline Apple Macintosh PowerBook 170 & Absoft -w -v -O -f -s & .23 & & \\
\hline Gould PN 6005 & VTX/32 2.0 Fortran 77 & .23 & & \\
\hline Harris 800 & Fortran 77 & .23 & & \\
\hline IBM $370 / 158$ & $\mathrm{H} \mathrm{opt}=3$ & .23 & & \\
\hline IBM 370/158 & VS Fortran opt $=3$ & .22 & & \\
\hline NORSK DATA ND-560 & Fortran-500 & .22 & & \\
\hline Celerity C1200 & UNIX 4.2 bsd f77 & .21 & & \\
\hline Honeywell DPS 8/70 & FR7X & .21 & & \\
\hline Denelcor HEP & f77 UPX & .21 & & \\
\hline VAX 11/785 FPA & VMS v4.5 & .20 & & \\
\hline CDC CYBER 170-720 & FTN 5, opt=2 & .20 & & \\
\hline Apple Macintosh IIsi & Absoft -w -v -O -f -s & .19 & & \\
\hline
\end{tabular}




\begin{tabular}{|c|c|c|c|c|}
\hline Computer & $\begin{array}{c}\text { "LINPACK Benchmark" } \\
\text { OS/Compiler }\end{array}$ & $\begin{array}{c}n=100 \\
\text { Mflop/s }\end{array}$ & $\begin{array}{c}\text { "TPP" } \\
\text { Best } \\
\text { Effort } \\
\text { n=1000 } \\
\text { Mflop/s }\end{array}$ & $\begin{array}{c}\text { "Theoritical } \\
\text { Peak" } \\
\text { Mflop/s }\end{array}$ \\
\hline Itel $\mathrm{AS} / 5 \bmod 3$ & $\mathrm{H}$ & .19 & & \\
\hline NORSK DATA ND-500 & Fortran-500-E & .19 & & \\
\hline KONTRON KSM/386 & UNIX SVS F77 2.8 & .19 & & \\
\hline Sun $386 \mathrm{i} / 25025 \mathrm{MHz}$ & SunOS 4.0; Sun 1.1 -O & .19 & & \\
\hline CDC CYBER 170-825 & FTN 5, opt=2 & .19 & & \\
\hline IBM 4341 MG10 & VS Fortran opt=3 & .19 & & \\
\hline Apollo DN2500 & & .18 & & \\
\hline Pyramid 98xe & OSx 4.0 & .18 & & \\
\hline IBM 9370-40 & VS Fortran $1.4 .0 \mathrm{opt}=3$ & .18 & & \\
\hline VAX 11/785 FPA & UNIX 4.2 bsd f77 & .18 & & \\
\hline DEC VAX 8250/8350 (UP) & VMS v4.6 & .18 & & \\
\hline CDC CYBER 170-825 & FTN 5, opt=1 & .18 & & \\
\hline Ridge Server/RT EFP & ROS/rf & .18 & & \\
\hline CDC CYBER 170-720 & FTN 5, opt=1 & .17 & & \\
\hline Ridge 32/130 & OS 3.3/RISC & .17 & & \\
\hline PC Craft $2400 / 25 \mathrm{MHz}$ w/80387 & PLI Fortran 2.09 & .17 & & \\
\hline Concurrent 3252 & OS 6.2 .4 fortran $\mathrm{z}$ & .17 & & \\
\hline Tandy $5000 \mathrm{MC} 20 \mathrm{MHz}$ & LPI Fortran 3.0 & .17 & & \\
\hline Tektronix $4315 \mathrm{w} / 68882$ & UTEK f77 & .17 & & \\
\hline CDC CYBER 180-810 & NOS/VE OPT=HIGH & .17 & & \\
\hline Prime P2755 & f77 rev 20.2.b2 -opt & .17 & & \\
\hline Apple Macintosh IIx & A/UX $2.0 \mathrm{f} 77$ & .16 & & \\
\hline Concurrent 3242 & OS 32 v7.2 f77 & .16 & & \\
\hline Compaq $386 / 20$ w/387 & Microsoft Fortran 4.1 & .16 & & \\
\hline Apple Macintosh IIcx & Absoft -w -v -O -f -s & .15 & & \\
\hline Apple Macintosh IIx & Absoft -w -v -O -f -s & .15 & & \\
\hline DEC VAX 8200/8300 & VMS v4.5 & .15 & & \\
\hline IBM PS/2-70 (20 MHz) & AIX 1.2 & .15 & & \\
\hline Apple Macintosh SE 30 & Absoft -w -v -O -f -s & .14 & & \\
\hline Apollo DN4000 & DOMAIN/IX SR9.7 opt 4 & .14 & & \\
\hline ICL 2988 & $\mathrm{f} 77 \mathrm{OPT}=2$ & .14 & & \\
\hline IBM 9370-20 & VS Fortran $1.4 .0 \mathrm{opt}=3$ & .14 & & \\
\hline HP Vectra RS/20C $20 \mathrm{MHz}$ & LPI Fortran 3.0 & .14 & & \\
\hline VAX 11/780 FPA & VMS v4.5 & .14 & & \\
\hline
\end{tabular}




\begin{tabular}{|c|c|c|c|c|}
\hline Computer & $\begin{array}{c}\text { "LINPACK Benchmark" } \\
\text { OS/Compiler }\end{array}$ & $\begin{array}{c}n=100 \\
\text { Mflop/s }\end{array}$ & $\begin{array}{c}\text { “TPP" } \\
\text { Best } \\
\text { Effort } \\
\text { n=1000 } \\
\text { Mflop/s }\end{array}$ & $\begin{array}{c}\text { "Theoritical } \\
\text { Peak" } \\
\text { Mflop/s }\end{array}$ \\
\hline Compaq 386/20 w/387 & RM/Forrtan 2.43 & .13 & & \\
\hline microVAX II & VMS v4.5 & .13 & & \\
\hline Prime P2450 & f77 rev 20.2.b2 -opt & .13 & & \\
\hline Apple Macintosh IIsi & Fortran & .12 & & \\
\hline Apple Mac II/16 Mhz/25 Mhz 68882 & Absoft $2.4-\mathrm{w}-\mathrm{v}-\mathrm{O}-\mathrm{f}-\mathrm{s}$ & .12 & & \\
\hline CDC 6500 & FUN & .12 & & \\
\hline CONCEPT 32/6750 & $\mathrm{UTX} / 32$ & .12 & & \\
\hline IBM PS/2-70 (16 MHz) & AIX 1.2 & .12 & & \\
\hline IBM RT w/68881 & $\mathrm{f} 77$ & .12 & & \\
\hline VAX 11/750 FPA & VMS v4.1 & .12 & & \\
\hline micro VAX II & ULTRIX 2.2/VFU & .12 & & \\
\hline Concurrent 3230 & OS 6.2 .2 fortran 5.2 & .11 & & \\
\hline Definicon DSI-780 & SVS Fortran (MSDOS) & .11 & & \\
\hline ENCORE Multimax NS32332 & $\mathrm{f} 77$ & .11 & & \\
\hline HP 9000 Series 350 & HP-UX, f77 5.2 & .11 & & \\
\hline Northgate $386 / 387(25 \mathrm{MHz})$ & Lahey F77, 4.00 & .11 & & \\
\hline Prime 750 & Primos f77 v19.1 & .11 & & \\
\hline Sun 3/260, 20 MHz 68881 & $3.2 \mathrm{f} 77-\mathrm{O}-\mathrm{f} 68881$ & .11 & & \\
\hline Tektronix 4315 w/68881 & UTEK $\mathrm{f77}$ & .11 & & \\
\hline VAX 11/780 FPA & UNIX 4.3 BSD f77 -O & .11 & & \\
\hline Sun 3/160, 16.7 MHz 68881 & $3.2 \mathrm{f} 77-\mathrm{O}-\mathrm{f} 68881$ & .10 & & \\
\hline NCUBE (1 proc. $8 \mathrm{MHz})$ & Fortran & .10 & & \\
\hline Apple Mac SE/30 & ABSOFT 2.4 & .10 & & \\
\hline Apollo DN590 & DOMAIN/IX SR9.7 opt 4 & .099 & & \\
\hline Masscomp MC5600 68881 & $\mathrm{f} 77 \mathrm{v} 1.2-\mathrm{O} 3 \mathrm{rtv}$ v3.1 & .099 & & \\
\hline VAX 11/750 FPA & UNIX 4.2 bsd f77 & .096 & & \\
\hline Prime 850 & Primos & .095 & & \\
\hline Sperry $1100 / 60$ & FTN opt=ZEO & .093 & & \\
\hline Pyramid 90X FPA & UNIX 4.2 bsd $\mathrm{f} 77$ & .088 & & \\
\hline Apple Mac II/16 Mhz/25 Mhz 68882 & Absoft 2.4 & .087 & & \\
\hline SUN-3/50, 16.7 MHz 68881 & $3.2 \mathrm{f} 77-\mathrm{O}-\mathrm{f} 68881$ & .087 & & \\
\hline HP 9000 Series 330 & HP-UX, f77 5.2 & .087 & & \\
\hline Apple Macintosh II & Absoft -w -v -O -f -s & .083 & & \\
\hline microVAX II & f77 Ultrix 1.1 & .082 & & \\
\hline
\end{tabular}




\begin{tabular}{|c|c|c|c|c|}
\hline Computer & $\begin{array}{c}\text { "LINPACK Benchmark" } \\
\text { OS/Compiler }\end{array}$ & $\begin{array}{c}n=100 \\
\text { Mflop/s }\end{array}$ & $\begin{array}{c}\text { “TPP" } \\
\text { Best } \\
\text { Effort } \\
\text { n=1000 } \\
\text { Mflop/s }\end{array}$ & $\begin{array}{l}\text { "Theoritical } \\
\text { Peak" } \\
\text { Mflop/s }\end{array}$ \\
\hline Apple Mac SE + 20 MHz 68881 & ABSOFT 2.4 & .082 & & \\
\hline Ridge $32 / 110$ & ROS 3.3/RISC & .081 & & \\
\hline Data General MV/8000 & f77 opt level 2 & .078 & & \\
\hline Apple MAC II w/882 & & .078 & & \\
\hline Prime P2350 & f77 rev 20.2.b2 -opt & .077 & & \\
\hline Apple Mac/Levco Prodigy 4 & ABSOFT MacFort 020 & .076 & & \\
\hline Apple Mac II w/68020 & FORTRAN & .074 & & \\
\hline HP 9000 Series 320 & HP-UX, f77 5.2 & .073 & & \\
\hline Apollo DN3000 & DOMAIN/IX SR9.7 opt 4 & .071 & & \\
\hline Apollo DN460/660 & AEGIS $8.0 \mathrm{FTN}$ & .069 & & \\
\hline Masscomp MC500 w/FPP & 3.1 Fortran & .061 & & \\
\hline Harris HS-20 w/FPP & Fortran 773.1 & .061 & & \\
\hline Sequent Balance 8000 & DYNIX Fortran 2.4.4 & .059 & & \\
\hline Definicon DSI-32/10 & Greenhills f77 (MSDOS) & .057 & & \\
\hline VAX $11 / 750$ & VMS v4.1 & .057 & & \\
\hline Encore Multimax & $\mathrm{f} 77$ & .055 & & \\
\hline HP 9000 Series 500 & Fortran 1.7 & .043 & & \\
\hline Opus 32.32 & UNIX, f77 4.2 bsd & .043 & & \\
\hline ATT 3B20 FP & UNIX V 2.0/4 & .040 & & \\
\hline Acorn Cambridge & fortran & .039 & & \\
\hline IBM 4331 MG2 & $\mathrm{H} \mathrm{opt}=3$ & .038 & & \\
\hline Burroughs B6800 & Fortran 77 ver 34 & .037 & & \\
\hline VAX 11/725 FPA & VMS v4.1 & .037 & & \\
\hline Masscomp MCS-541 w/FPB & Fortran 3.1 & .037 & & \\
\hline IBM RT PC Model 20 & f77 & .036 & & \\
\hline VAX 11/730 FPA & VMS & .036 & & \\
\hline Prime 2250 & Fortran 77 & .034 & & \\
\hline IBM PC-AT/370 & VS Fortran opt $=3$ & .033 & & \\
\hline IBM PC-XT/370 & $\mathrm{H} \mathrm{opt}=3$ & .031 & & \\
\hline VAX $11 / 750$ & UNIX 4.2 bsd f77 & .029 & & \\
\hline Apollo DN320 & AEGIS $8.0 \mathrm{FTN}$ & .028 & & \\
\hline Sun $2 / 50+$ SKY FFP & f77 -O -fsky 3.0 & .027 & & \\
\hline Ametek S14/32 (1 node) & RM Fortran 2.11 & .026 & & \\
\hline Apollo DN550 FPA & AEGIS $8.0 \mathrm{FTN}$ & .025 & & \\
\hline
\end{tabular}




\begin{tabular}{|c|c|c|c|c|}
\hline Computer & $\begin{array}{c}\text { "LINPACK Benchmark" } \\
\text { OS/Compiler }\end{array}$ & $\begin{array}{c}n=100 \\
\text { Mflop/s }\end{array}$ & $\begin{array}{c}\text { “TPP" } \\
\text { Best } \\
\text { Effort } \\
\text { n=1000 } \\
\text { Mflop/s }\end{array}$ & $\begin{array}{l}\text { "Theoritical } \\
\text { Peak" } \\
\text { Mflop/s }\end{array}$ \\
\hline AMSTRAC $15128086 / 80879.54 \mathrm{MHz}$ & MS-Fortran $4.0-\mathrm{Ox}-\mathrm{AH}$ & .022 & & \\
\hline microVAX I & VMS & .023 & & \\
\hline Canaan & VS & .021 & & \\
\hline Chas. River Data $6835+$ SKY & SVS Fortran 77 & .018 & & \\
\hline Apollo DN 420 PEB & AEGIS 7+ FTN & .017 & & \\
\hline IBM AT w/80287 & PROFORT 1.0 & .012 & & \\
\hline IBM PC w/8087 & PROFORT 1.0 & .012 & & \\
\hline Cadtrak DS1/8087 & Intel Fortran 77 & .011 & & \\
\hline Apple Mac Classic II/16 MHz68030 & Absoft 2.4 & .011 & & \\
\hline IBM PC/AT w/80287 & Microsoft 3.2 & .0091 & & \\
\hline Chas. River Data 6835 & SVS Fortran 77 & .0088 & & \\
\hline Apollo DN300 & AEGIS $8.0 \mathrm{FTN}$ & .0071 & & \\
\hline Masscomp MC500 & 3.1 Fortran & .0070 & & \\
\hline IBM PC w/8087 & Microsoft 3.2 & .0069 & & \\
\hline Apple Mac II & ABSOFT 2.4 & .0064 & & \\
\hline HP 9000 Series 200 & HP-UX & .0062 & & \\
\hline Sun $2 / 50$ & f77 -O -fsoft 3.0 & .0055 & & \\
\hline Atari ST & ABSOFT AC/Fortran v2.2 & .0051 & & \\
\hline Apple Macintosh & ABSOFT 2.0b & .0038 & & \\
\hline Palm Pilot III & & .00169 & & \\
\hline
\end{tabular}




\section{Table 2: A Look at Parallel Processing}

1000 x 1000 Problem with Parallel Processing

\begin{tabular}{|c|c|c|c|c|c|}
\hline & $\begin{array}{c}\text { Time } \\
\text { uniprocessor }\end{array}$ & $\begin{array}{l}\text { no. of } \\
\text { procs }\end{array}$ & $\begin{array}{c}\text { Time } \\
\text { multiprocs }\end{array}$ & Speedup & Efficiency \\
\hline Hitachi S-3800/480 & 0.104 & 4 & .0324 & 3.21 & .80 \\
\hline Hitachi S-3800/380 & 0.104 & 3 & .0396 & 2.63 & .88 \\
\hline Hitachi S-3800/280 & 0.104 & 2 & .0549 & 1.89 & .95 \\
\hline NEC SX-3/*4R & 0.128 & 4 & .0442 & 2.91 & .73 \\
\hline $\mathrm{NEC} \mathrm{SX-3/*4R}$ & 0.128 & 2 & .0707 & 1.82 & .91 \\
\hline NEC SX-3/*4 & 0.148 & 4 & .0498 & 2.98 & .74 \\
\hline NEC SX-3/*4 & 0.148 & 2 & .0821 & 1.81 & .90 \\
\hline $\mathrm{NEC} \mathrm{SX-3/*2 \textrm {R }}$ & 0.243 & 4 & .0747 & 3.25 & .81 \\
\hline $\mathrm{NEC} \mathrm{SX}-3 / * 2 \mathrm{R}$ & 0.243 & 2 & .1307 & 1.86 & .93 \\
\hline NEC SX-3/*2 & 0.293 & 4 & .0863 & 3.40 & .85 \\
\hline NEC SX-3/*2 & 0.293 & 2 & .1518 & 1.93 & .96 \\
\hline Cray C90 & 0.740 & 16 & .0618 & 11.95 & .75 \\
\hline Cray C90 & 0.740 & 8 & .108 & 6.85 & .86 \\
\hline Cray C90 & 0.740 & 4 & .204 & 3.63 & .91 \\
\hline Cray C90 & 0.740 & 2 & .392 & 1.89 & .94 \\
\hline NEC SX-3 & 0.149 & 2 & .0820 & 1.82 & .91 \\
\hline NEC SX-3/*1R & 0.472 & 4 & .139 & 3.40 & .85 \\
\hline NEC SX-3/*1R & 0.472 & 2 & .255 & 1.85 & .93 \\
\hline Convex C4/XA & 0.949 & 4 & .264 & 3.59 & .90 \\
\hline Convex C4/XA & 0.949 & 3 & .346 & 2.74 & .91 \\
\hline Convex $\mathrm{C} 4 / \mathrm{XA}$ & 0.949 & 2 & .501 & 1.89 & .95 \\
\hline IBM ES/9000 (7.1 ns) & 1.58 & 8 & .293 & 5.34 & .67 \\
\hline IBM ES/9000 (7.1 ns) & 1.58 & 7 & .322 & 4.91 & .70 \\
\hline IBM ES/9000 (7.1 ns) & 1.58 & 6 & .347 & 4.56 & .76 \\
\hline IBM ES/9000 (7.1 ns) & 1.58 & 5 & .397 & 3.98 & .80 \\
\hline IBM ES/9000 (7.1 ns) & 1.58 & 4 & .485 & 3.26 & .82 \\
\hline IBM ES/9000 (7.1 ns) & 1.58 & 3 & .617 & 2.56 & .85 \\
\hline IBM ES/9000 (7.1 ns) & 1.58 & 2 & .871 & 1.82 & .91 \\
\hline Cray Y-MP/8 & 2.17 & 8 & .312 & 6.96 & .87 \\
\hline Cray Y-MP/8 & 2.17 & 4 & .577 & 3.76 & .94 \\
\hline Cray Y-MP/8 & 2.17 & 3 & .754 & 2.88 & .96 \\
\hline Cray Y-MP/8 & 2.17 & 2 & 1.11 & 1.96 & .98 \\
\hline Cray Y-MP/98 & 2.17 & 8 & .386 & 5.65 & .71 \\
\hline Cray Y-MP/98 & 2.17 & 4 & .600 & 3.63 & .91 \\
\hline Cray Y-MP/98 & 2.17 & 2 & 1.12 & 1.94 & .97 \\
\hline IBM ES/9000 (9 ns) & 1.98 & 6 & .458 & 4.31 & .72 \\
\hline IBM ES/9000 (9 ns) & 1.98 & 5 & .552 & 3.58 & .72 \\
\hline IBM ES/9000 (9 ns) & 1.98 & 4 & .666 & 2.97 & .74 \\
\hline IBM ES/9000 (9 ns) & 1.98 & 3 & .862 & 2.29 & .76 \\
\hline IBM ES/9000 (9 ns) & 1.98 & 2 & 1.24 & 1.59 & .80 \\
\hline Cray 2S & 1.76 & 4 & .476 & 3.66 & .91 \\
\hline Cray $2 \mathrm{~S}$ & 1.76 & 3 & .617 & 2.82 & .94 \\
\hline
\end{tabular}


Computer

1000 x 1000 Problem with Parallel Processing

\begin{tabular}{|c|c|c|c|c|c|}
\hline & $\begin{array}{c}\text { Time } \\
\text { uniprocessor }\end{array}$ & $\begin{array}{l}\text { no. of } \\
\text { procs }\end{array}$ & $\begin{array}{c}\text { Time } \\
\text { multiprocs }\end{array}$ & Speedup & Efficiency \\
\hline Cray $2 \mathrm{~S}$ & 1.76 & 2 & .902 & 1.93 & .96 \\
\hline Cray X-MP/4 & 3.10 & 4 & .813 & 3.78 & .94 \\
\hline Cray X-MP/4 & 3.10 & 3 & 1.07 & 2.87 & .96 \\
\hline Cray X-MP/4 & 3.10 & 2 & 1.57 & 1.96 & .98 \\
\hline Convex C3880 & 5.90 & 8 & .841 & 7.02 & .88 \\
\hline Convex C3840 & 5.90 & 4 & 1.58 & 3.74 & .94 \\
\hline Convex C3830 & 5.90 & 3 & 2.05 & 2.88 & .96 \\
\hline Convex C3820 & 5.90 & 2 & 3.01 & 1.96 & .98 \\
\hline DEC 10000 Alpha & 4.31 & 6 & .889 & 4.85 & .81 \\
\hline DEC 10000 Alpha & 4.31 & 5 & 1.04 & 4.12 & .82 \\
\hline DEC 10000 Alpha & 4.31 & 4 & 1.28 & 3.37 & .84 \\
\hline DEC 10000 Alpha & 4.31 & 3 & 1.66 & 2.60 & .87 \\
\hline DEC 10000 Alpha & 4.31 & 2 & 2.44 & 1.76 & .88 \\
\hline Convex SPP-1000 & i 5.45 & 8 & 0.8905 & 6.120 & .77 \\
\hline Convex SPP-1000 & i 5.45 & 4 & 1.513 & 3.602 & .90 \\
\hline Convex SPP-1000 & i 5.45 & 2 & 2.628 & 2.073 & 1.03 \\
\hline Cray S-MP/MCP784 & 21.4 & 84 & .902 & 23.7 & .28 \\
\hline Cray S-MP/MCP756 & 21.4 & 56 & .986 & 21.7 & .39 \\
\hline Cray S-MP/MCP728 & 21.4 & 28 & 1.32 & 16.2 & .58 \\
\hline Cray S-MP/MCP707 & 21.4 & 7 & 3.46 & 6.19 & .88 \\
\hline DEC 7000 Alpha & 4.74 & 6 & .978 & 4.84 & .81 \\
\hline DEC 7000 Alpha & 4.74 & 5 & 1.14 & 4.16 & .83 \\
\hline DEC 7000 Alpha & 4.74 & 4 & 1.38 & 3.43 & .86 \\
\hline DEC 7000 Alpha & 4.74 & 3 & 1.81 & 2.62 & .87 \\
\hline DEC 7000 Alpha & 4.74 & 2 & 2.67 & 1.77 & .89 \\
\hline Meiko CS2 & 6.89 & 64 & 1.03 & 6.69 & .10 \\
\hline Meiko CS2 & 6.89 & 32 & 1.03 & 6.69 & .21 \\
\hline Meiko CS2 & 6.89 & 16 & 1.26 & 5.47 & .34 \\
\hline Meiko CS2 & 6.89 & 8 & 1.59 & 4.33 & .54 \\
\hline Meiko CS2 & 6.89 & 4 & 2.32 & 2.97 & .74 \\
\hline Meiko CS2 & 6.89 & 2 & 3.96 & 1.74 & .87 \\
\hline Fujitsu AP1000 & 160 & 512 & 1.10 & 147 & .29 \\
\hline Fujitsu AP1000 & 160 & 256 & 1.50 & 108 & .42 \\
\hline Fujitsu AP1000 & 160 & 128 & 2.42 & 66.5 & .52 \\
\hline Fujitsu AP1000 & 160 & 64 & 3.51 & 46.0 & .72 \\
\hline Fujitsu AP1000 & 160 & 32 & 6.71 & 24.0 & .75 \\
\hline Fujitsu AP1000 & 160 & 16 & 11.5 & 13.9 & .87 \\
\hline Fujitsu AP1000 & 160 & 8 & 22.6 & 7.12 & .89 \\
\hline Fujitsu AP1000 & 160 & 4 & 41.3 & 3.90 & .97 \\
\hline Fujitsu AP1000 & 160 & 2 & 81.4 & 1.96 & .98 \\
\hline IBM 3090/J (14.5 ns) & 6.8832 & 6 & 1.24 & 5.57 & .93 \\
\hline IBM 3090/J (14.5 ns) & 6.8832 & 5 & 1.46 & 4.72 & .94 \\
\hline IBM 3090/J (14.5 ns) & 6.8832 & 4 & 1.80 & 3.81 & .95 \\
\hline IBM 3090/J (14.5 ns) & 6.8832 & 3 & 2.35 & 2.93 & .98 \\
\hline
\end{tabular}


Computer

1000 x 1000 Problem with Parallel Processing

\begin{tabular}{|c|c|c|c|c|c|}
\hline & $\begin{array}{c}\text { Time } \\
\text { uniprocessor }\end{array}$ & $\begin{array}{l}\text { no. of } \\
\text { procs }\end{array}$ & $\begin{array}{c}\text { Time } \\
\text { multiprocs }\end{array}$ & Speedup & Efficiency \\
\hline IBM 3090/J (14.5 ns) & 6.8832 & 2 & 3.48 & 1.98 & .99 \\
\hline IBM 3090/600S VF & 7.27 & 6 & 1.29 & 5.64 & .94 \\
\hline IBM 3090/500S VF & 7.27 & 5 & 1.52 & 4.78 & .96 \\
\hline IBM 3090/400S VF & 7.27 & 4 & 1.89 & 3.85 & .96 \\
\hline IBM 3090/300S VF & 7.27 & 3 & 2.46 & 2.96 & .99 \\
\hline IBM 3090/280S VF & 7.27 & 2 & 3.65 & 1.99 & .99 \\
\hline IBM 3090/200S VF & 7.27 & 2 & 3.64 & 1.99 & .99 \\
\hline Kendall Square Research & 21.5 & 32 & 1.30 & 16.5 & .52 \\
\hline Kendall Square Research & 21.5 & 16 & 2.17 & 9.90 & .62 \\
\hline Kendall Square Research & 21.5 & 8 & 4.57 & 4.71 & .59 \\
\hline Kendall Square Research & 21.5 & 4 & 14.2 & 1.52 & .38 \\
\hline IBM ES/9000 (11 ns) & 5.14 & 4 & 1.51 & 3.39 & .85 \\
\hline IBM ES/9000 (11 ns) & 5.14 & 3 & 1.90 & 2.71 & .90 \\
\hline IBM ES/9000 (11 ns) & 5.14 & 2 & 2.74 & 1.88 & .94 \\
\hline Intel Delta & 22 & 512 & 1.5 & 14.7 & .03 \\
\hline Intel Delta & 22 & 256 & 1.6 & 13.8 & .05 \\
\hline Intel Delta & 22 & 128 & 1.7 & 12.9 & .10 \\
\hline Intel Delta & 22 & 64 & 1.9 & 11.5 & .18 \\
\hline Intel Delta & 22 & 32 & 2.2 & 10.0 & .31 \\
\hline Intel Delta & 22 & 16 & 2.9 & 7.59 & .47 \\
\hline Intel Delta & 22 & 8 & 4.1 & 5.37 & .67 \\
\hline Intel Delta & 22 & 4 & 6.7 & 3.28 & .82 \\
\hline Intel Delta & 22 & 2 & 11.6 & 1.90 & .95 \\
\hline IBM 3090/600E VF & 9.36 & 6 & 1.73 & 5.41 & .90 \\
\hline IBM 3090/500E VF & 9.36 & 5 & 2.02 & 4.63 & .93 \\
\hline IBM 3090/400E VF & 9.36 & 4 & 2.48 & 3.77 & .94 \\
\hline IBM 3090/300E VF & 9.36 & 3 & 3.21 & 2.92 & .97 \\
\hline IBM 3090/200E VF & 9.36 & 2 & 4.73 & 1.98 & .99 \\
\hline Sun Sparc2000(50 MHz) & 23.85 & 16 & 2.01 & 11.89 & .74 \\
\hline Sun Sparc2000(50 MHz) & 23.85 & 12 & 2.26 & 10.54 & .88 \\
\hline Sun Sparc2000(50 MHz) & 23.85 & 8 & 2.99 & 7.96 & .99 \\
\hline Alliant FX/2800-200 & 22.9 & 14 & 2.06 & 11.1 & .79 \\
\hline Alliant FX/2800-200 & 22.9 & 12 & 2.30 & 10.0 & .83 \\
\hline Alliant FX/2800-200 & 22.9 & 10 & 2.68 & 8.54 & .85 \\
\hline Alliant FX/2800-200 & 22.9 & 8 & 3.24 & 7.07 & .88 \\
\hline Alliant FX/2800-200 & 22.9 & 4 & 6.07 & 3.77 & .94 \\
\hline Alliant FX/2800-200 & 22.9 & 2 & 11.8 & 1.94 & .97 \\
\hline IBM PVS & 20.4 & 32 & 2.17 & 9.35 & .29 \\
\hline IBM PVS & 20.4 & 16 & 2.35 & 8.64 & .54 \\
\hline IBM PVS & 20.4 & 8 & 3.41 & 5.95 & .74 \\
\hline IBM PVS & 20.4 & 4 & 5.71 & 3.56 & .89 \\
\hline IBM PVS & 20.4 & 2 & 10.6 & 1.92 & .96 \\
\hline IBM RS/6000 Cluster (62.5 ns) & 7.42 & 8 & 2.48 & 2.99 & .37 \\
\hline IBM RS/6000 Cluster (62.5 ns) & 7.42 & 4 & 3.24 & 2.29 & .57 \\
\hline
\end{tabular}


Computer

1000 x 1000 Problem with Parallel Processing

\begin{tabular}{|c|c|c|c|c|c|}
\hline & $\begin{array}{c}\text { Time } \\
\text { uniprocessor }\end{array}$ & $\begin{array}{l}\text { no. of } \\
\text { procs }\end{array}$ & $\begin{array}{c}\text { Time } \\
\text { multiprocs }\end{array}$ & Speedup & Efficiency \\
\hline IBM RS/6000 Cluster (62.5 ns) & 7.42 & 2 & 4.64 & 1.60 & .80 \\
\hline nCUBE 2 & 331 & 1024 & 2.59 & 128 & .12 \\
\hline nCUBE 2 & 331 & 512 & 3.29 & 101 & .20 \\
\hline nCUBE 2 & 331 & 256 & 4.05 & 81.7 & .32 \\
\hline nCUBE 2 & 331 & 128 & 5.74 & 57.7 & .45 \\
\hline nCUBE 2 & 331 & 64 & 8.70 & 38.0 & .59 \\
\hline nCUBE 2 & 331 & 32 & 14.5 & 22.8 & .71 \\
\hline nCUBE 2 & 331 & 16 & 25.6 & 12.9 & .81 \\
\hline nCUBE 2 & 331 & 8 & 46.9 & 7.04 & .88 \\
\hline nCUBE 2 & 331 & 4 & 89.1 & 3.71 & .93 \\
\hline nCUBE 2 & 331 & 2 & 171. & 1.93 & .97 \\
\hline Intel iPSC/860 & 22 & 128 & 2.8 & 7.68 & .06 \\
\hline Intel iPSC/860 & 22 & 64 & 3.2 & 6.72 & .11 \\
\hline Intel iPSC/860 & 22 & 32 & 4.0 & 5.38 & .17 \\
\hline Intel iPSC/860 & 22 & 16 & 5.1 & 4.22 & .26 \\
\hline Intel iPSC/860 & 22 & 8 & 6.5 & 3.31 & .41 \\
\hline Intel iPSC/860 & 22 & 4 & 8.9 & 2.42 & .60 \\
\hline Intel iPSC/860 & 22 & 2 & 12.8 & 1.68 & .84 \\
\hline $\begin{array}{l}\text { Meiko Computing Surface } \\
\text { (i860) }\end{array}$ & 21.9 & 32 & 3.19 & 6.85 & .21 \\
\hline $\begin{array}{l}\text { Meiko Computing Surface } \\
\text { (i860) }\end{array}$ & 21.9 & 24 & 3.30 & 6.62 & .28 \\
\hline $\begin{array}{l}\text { Meiko Computing Surface } \\
\text { (i860) }\end{array}$ & 21.9 & 16 & 3.57 & 6.12 & .38 \\
\hline $\begin{array}{l}\text { Meiko Computing Surface } \\
\text { (i860) }\end{array}$ & 21.9 & 8 & 4.56 & 4.79 & .60 \\
\hline $\begin{array}{l}\text { Meiko Computing Surface } \\
\text { (i860) }\end{array}$ & 21.9 & 4 & 6.83 & 3.20 & .80 \\
\hline $\begin{array}{l}\text { Meiko Computing Surface } \\
\text { (i860) }\end{array}$ & 21.9 & 2 & 11.6 & 1.88 & .94 \\
\hline IBM RS/6000 Cluster (50 ns) & 7.95 & 8 & 3.44 & 2.31 & .29 \\
\hline IBM RS/6000 Cluster (50 ns) & 7.95 & 6 & 3.84 & 2.07 & .35 \\
\hline IBM RS/6000 Cluster (50 ns) & 7.95 & 4 & 4.39 & 1.81 & .45 \\
\hline IBM RS/6000 Cluster (50 ns) & 7.95 & 2 & 6.02 & 1.32 & .66 \\
\hline Sun Sparc2000(50 MHz) & 26.71 & 8 & 3.37 & 7.92 & .99 \\
\hline Sun Sparc2000(50 MHz) & 26.71 & 4 & 6.24 & 4.28 & 1.07 \\
\hline Sun Sparc2000(50 MHz) & 26.71 & 2 & 12.60 & 2.12 & 1.06 \\
\hline Convex C3240 & 14.9 & 4 & 3.92 & 3.81 & .95 \\
\hline Convex C3230 & 14.9 & 3 & 5.06 & 2.95 & .98 \\
\hline Convex C3220 & 14.9 & 2 & 7.50 & 1.99 & .99 \\
\hline Convex C-240 & 15 & 4 & 4.03 & 3.76 & .94 \\
\hline Convex C-230 & 15 & 3 & 5.20 & 2.91 & .97 \\
\hline Convex C-220 & 15 & 2 & 7.65 & 1.98 & .99 \\
\hline Parsytec FT-400 & 1075 & 400 & 4.90 & 219. & .55 \\
\hline Parsytec FT-400 & 1075 & 256 & 6.59 & 163. & .64 \\
\hline Parsytec FT-400 & 1075 & 100 & 13.2 & 81.4 & .81 \\
\hline
\end{tabular}


Computer

1000 x 1000 Problem with Parallel Processing

\begin{tabular}{|c|c|c|c|c|c|}
\hline & $\begin{array}{c}\text { Time } \\
\text { uniprocessor }\end{array}$ & $\begin{array}{l}\text { no. of } \\
\text { procs }\end{array}$ & $\begin{array}{c}\text { Time } \\
\text { multiprocs }\end{array}$ & Speedup & Efficiency \\
\hline Parsytec FT-400 & 1075 & 64 & 19.1 & 56.3 & .88 \\
\hline Parsytec FT-400 & 1075 & 16 & 69.2 & 15.5 & .97 \\
\hline Sun Sparc10/514(50 MHz) & 24.73 & 4 & 6.81 & 3.63 & .91 \\
\hline Sun Sparc10/514(50 MHz) & 24.73 & 2 & 11.71 & 2.11 & 1.06 \\
\hline FPS Model 522 & 12 & 2 & 6.36 & 1.89 & .95 \\
\hline Suprenum S1C1 & 51 & 16 & 6.4 & 8.0 & .50 \\
\hline Suprenum S1C1 & 51 & 14 & 7.1 & 7.2 & .51 \\
\hline Suprenum S1C1 & 51 & 12 & 7.9 & 6.5 & .54 \\
\hline Suprenum S1C1 & 51 & 10 & 8.9 & 5.8 & .58 \\
\hline Suprenum S1C1 & 51 & 8 & 10.4 & 4.9 & .61 \\
\hline Suprenum S1C1 & 51 & 6 & 13.1 & 3.9 & .65 \\
\hline Suprenum S1C1 & 51 & 4 & 18.1 & 2.8 & .70 \\
\hline Suprenum S1C1 & 51 & 2 & 33.4 & 1.5 & .75 \\
\hline Alliant FX/800-200 & 24.2 & 4 & 7.09 & 3.41 & .85 \\
\hline Alliant FX/800-200 & 24.2 & 2 & 12.7 & 1.91 & .95 \\
\hline Alliant FX/80 & 57.7 & 8 & 9.64 & 5.99 & .75 \\
\hline Alliant FX/80 & 57.7 & 7 & 10.6 & 5.44 & .78 \\
\hline Alliant FX/80 & 57.7 & 6 & 11.8 & 4.89 & .82 \\
\hline Alliant FX/80 & 57.7 & 5 & 13.6 & 4.24 & .85 \\
\hline Alliant FX/80 & 57.7 & 4 & 16.2 & 3.56 & .89 \\
\hline Alliant FX/80 & 57.7 & 3 & 20.7 & 2.79 & .93 \\
\hline Alliant FX/80 & 57.7 & 2 & 29.8 & 1.94 & .97 \\
\hline Stardent 1540 (Ardent Titan-4) & 51.2 & 4 & 14.3 & 3.57 & .89 \\
\hline Stardent 1530 (Ardent Titan-3) & 51.2 & 3 & 18.3 & 2.80 & .93 \\
\hline Stardent 1520 (Ardent Titan-2) & 51.2 & 2 & 26.3 & 1.95 & .97 \\
\hline SGI 4D/480 $40 \mathrm{MHz}$ & 54.0 & 8 & 9.48 & 5.70 & .71 \\
\hline SGI 4D/440 $40 \mathrm{MHz}$ & 54.0 & 4 & 15.91 & 3.39 & .85 \\
\hline SGI 4D/420 $40 \mathrm{MHz}$ & 54.0 & 2 & 28.80 & 1.88 & .94 \\
\hline SGI 4D/380 $33 \mathrm{MHz}$ & 65.0 & 8 & 11.13 & 5.84 & .73 \\
\hline SGI 4D/340 $33 \mathrm{MHz}$ & 65.0 & 4 & 18.62 & 3.49 & .87 \\
\hline SGI 4D/320 $33 \mathrm{MHz}$ & 65.0 & 2 & 34.17 & 1.90 & .95 \\
\hline Sun Sparc10/402(40 MHz) & 29.03 & 2 & 16.28 & 1.78 & .89 \\
\hline Alliant FX/40 & 66.1 & 4 & 20.5 & 3.22 & .81 \\
\hline Alliant FX/40 & 66.1 & 3 & 24.9 & 2.65 & .88 \\
\hline Alliant FX/40 & 66.1 & 2 & 34.8 & 1.90 & .95 \\
\hline SGI 4D/240 $25 \mathrm{MHz}$ & 85.2 & 4 & 23.89 & 3.57 & .89 \\
\hline SGI 4D/220 $25 \mathrm{MHz}$ & 85.2 & 2 & 44.89 & 1.90 & .95 \\
\hline Alliant FX/4 & 106 & 4 & 32.3 & 3.28 & .82 \\
\hline Alliant FX/4 & 106 & 3 & 38.7 & 2.74 & .91 \\
\hline Alliant FX/4 & 106 & 2 & 55.8 & 1.90 & .95 \\
\hline DEC VAX 6000-460 & 439 & 6 & 80 & 5.5 & .92 \\
\hline DEC VAX 6000-450 & 439 & 5 & 94 & 4.7 & .94 \\
\hline DEC VAX 6000-440 & 439 & 4 & 114 & 3.8 & .96 \\
\hline DEC VAX 6000-430 & 439 & 3 & 152 & 2.9 & .96 \\
\hline
\end{tabular}


Computer

1000 x 1000 Problem with Parallel Processing

\begin{tabular}{lccccc} 
& $\begin{array}{c}\text { Time } \\
\text { uniprocessor }\end{array}$ & $\begin{array}{c}\text { no. of } \\
\text { procs }\end{array}$ & $\begin{array}{c}\text { Time } \\
\text { multiprocs }\end{array}$ & Speedup & Efficiency \\
\hline DEC VAX 6000-420 & 439 & 2 & 222 & 1.9 & .99 \\
ELXSI 6420 & 475 & 5 & 104 & 4.57 & .91 \\
ELXSI 6420 & 475 & 3 & 167 & 2.84 & .95 \\
ELXSI 6420 & 475 & 2 & 245 & 1.94 & .97 \\
DEC VAX 6240 & 1295 & 4 & 332 & 3.90 & .98 \\
DEC VAX 6230 & 1295 & 3 & 439 & 2.95 & .98 \\
DEC VAX 6220 & 1295 & 2 & 654 & 1.98 & .99 \\
Sequent Balance 21000 & 11111 & 30 & 445 & 25.0 & .83
\end{tabular}




\section{Table 3: Highly Parallel Computing}

\section{Computer \\ (Full Precision)}

\begin{tabular}{|c|c|c|c|c|c|}
\hline $\begin{array}{c}\text { Computer } \\
\text { (Full Precision) }\end{array}$ & $\begin{array}{l}\text { Number } \\
\text { of Procs } \\
\text { or Cores }\end{array}$ & $\begin{array}{c}\boldsymbol{R}_{\max } \\
\text { GFlop/s }\end{array}$ & $\begin{array}{l}N_{\max } \\
\text { Order }\end{array}$ & $\begin{array}{c}N_{1 / 2} \\
\text { Order }\end{array}$ & $\begin{array}{c}\boldsymbol{R}_{\text {Peak }} \\
\text { GFlop/s }\end{array}$ \\
\hline $\begin{array}{l}\text { NUDT, Inspur Tianhe-2 (TH-2) Model } \\
\text { NH-IVB-FEP } \\
\text { 2.2GHz } 160002 \text { Intel Xeon IvyBridge (6 core) E5-2692 } \\
\text { Intel Xeon Phi 31S1P }\end{array}$ & 2371200 & 22808300 & 6974976 & & 41733120 \\
\hline $\begin{array}{l}\text { IBM Blue Gene/Q Power BCQ } 1.6 \mathrm{GHz} \text { (120 racks * } 1024 \\
\text { nodes/rack * } 16 \text { cores/node) w/Custom }\end{array}$ & 1966080 & 21466530 & 14942207 & & 25165824 \\
\hline $\begin{array}{l}\text { IBM Blue Gene/Q Power BCQ } 1.6 \mathrm{GHz} \text { (96 racks * } 1024 \\
\text { nodes/rack * } 16 \text { cores/node) w/custom }\end{array}$ & 1572864 & 16324751 & 12681215 & & 20132659 \\
\hline $\begin{array}{l}\text { IBM Blue Gene/Q Power BCQ } 1.6 \mathrm{GHz} \text { ( } 72 \text { racks * } 1024 \\
\text { nodes/rack * } 16 \text { cores/node) w/custom }\end{array}$ & 1179648 & 12003644 & 10715135 & & 15099494 \\
\hline $\begin{array}{l}\text { K computer, Fujitsu SPARC64 VIIIfx 2.0GHz, } 8 \text { core w/Tofu } \\
\text { interconnect }\end{array}$ & 705024 & 10510000 & 11870208 & & 11280384 \\
\hline $\begin{array}{l}\text { K computer, Fujitsu SPARC64 VIIIfx 2.0GHz, } 8 \text { core w/Tofu } \\
\text { interconnect }\end{array}$ & 548352 & 8162000 & 10725120 & & 8773632 \\
\hline $\begin{array}{l}\text { IBM Blue Gene/Q Power BCQ } 1.6 \mathrm{GHz} \text { ( } 48 \text { racks * } 1024 \\
\text { nodes/rack * } 16 \text { cores/node) w/custom }\end{array}$ & 786432 & 8152590 & 8912895 & & 10066330 \\
\hline $\begin{array}{l}\text { IBM Blue Gene/Q Power BCQ } 1.6 \mathrm{GHz}(24 \text { racks * } 1024 \\
\text { nodes/rack * } 16 \text { cores/node) w/custom }\end{array}$ & 393216 & 4141180 & 6422527 & & 5033165 \\
\hline $\begin{array}{l}\text { IBM iDataPlex dx360 M4 } 2 \text { x Intel E5-2680v2 }(2.8 \mathrm{GHz}) \text { Ivy } \\
\text { Bridge CPU Cores: } 26,100 \text { (1305 nodes * } 2 \text { sockets * } 10 \\
\text { cores/socket) GPUs: NVIDIA } 2 \text { x K20x -GPU cores: } 36,540 \\
\text { InfiniBand FDR }\end{array}$ & 62640 & 3003000 & 3838464 & & 4003740 \\
\hline $\begin{array}{l}\text { IBM iDataPlex DX360M4 Intel Sandybridge } 2.7 \mathrm{GHz}(9216 \\
\text { nodes * } 2 \text { sockets * } 8 \text { cores/socket) w/InfiniBand }\end{array}$ & 147456 & 2897000 & 5201920 & & 3185050 \\
\hline $\begin{array}{l}\text { TH-1A (14336 6-core Intel X5670 } 2.93 \mathrm{GHz}+7168 \text { Nvidia } \\
\text { M2050 w/custom interconnect) }\end{array}$ & 186368 & 2566000 & 3600000 & 1000000 & 4701061 \\
\hline $\begin{array}{l}\text { IBM iDataPlex DX360M4 Intel Sandybridge } 2.7 \mathrm{GHz} \text { ( } 7168 \\
\text { nodes * } 2 \text { sockets * } 8 \text { cores/socket) w/InfiniBand }\end{array}$ & 114688 & 2072000 & 4464640 & & 2477261 \\
\hline $\begin{array}{l}\text { IBM Power } 775 \text { (IBM POWER7 } 3.836 \mathrm{GHz} \text { w/Custom } \\
\text { (equivlent to } 247.5 \text { drawers x } 8 \text { sockets per drawer x } 32 \text { cores } \\
\text { per socket) ) }\end{array}$ & 63360 & 1515000 & 2280960 & & 1944392 \\
\hline $\begin{array}{l}\text { IBM Power } 775 \text { (IBM POWER7 } 3.836 \mathrm{GHz})(216 \text { drawers x } \\
8 \text { sockets per drawer x } 32 \text { cores per socket) Custom }\end{array}$ & 55296 & 1429000 & 4147200 & & 1696923 \\
\hline $\begin{array}{l}\text { IBM Blue Gene/Q Power BCQ } 1.6 \mathrm{GHz} \text { ( } 8 \text { racks * } 1024 \\
\text { nodes/rack * } 16 \text { cores/node) w/custom }\end{array}$ & 131072 & 1358197 & 3899391 & & 1677721 \\
\hline $\begin{array}{l}464 \text { Dawning TC3600 Blade System } 4640 \text { Computing Nodes } \\
(2 * \text { Intel } 6 \text { core X5650 } 2.666 \mathrm{GHz}, 1 * \text { NVidia Tesla C } 2050 \\
\text { GPU) w/InfiniBand }\end{array}$ & 120640 & 1271000 & 2359296 & & 2983520 \\
\hline $\begin{array}{l}\text { IBM Power } 775 \text { (POWER7 } 3.836 \mathrm{GHz} \text {, w/custom) (8 sockets } \\
\text { per drawer x } 32 \text { cores per socket) }\end{array}$ & 47488 & 1183000 & 3419136 & & 1457311 \\
\hline $\begin{array}{l}\text { IBM BladeCenter cluster of } 3240 \text { nodes dual socket } 1.8 \mathrm{GHz} \\
\text { Opteron (dual core) LS21 blades plus } 6480 \text { nodes dual socket } \\
3.2 \mathrm{GHz} \text { PowerXCell } 8 \mathrm{i} \text { ( } 8 \mathrm{SPU}+1 \text { PPU cores) QS22 blades } \\
\text { w/InfiniBand }\end{array}$ & 129600 & 1105000 & 2329599 & & 1456704 \\
\hline $\begin{array}{l}\text { TSUBAME } 2.0 ; 1357 \text { HP Proliant SL390s G7 nodes w/ Xeon } \\
\text { X5670 (2.93GHz) } 6 \text { cores x 2sockets, NVIDIA Tesla M2050 } \\
\text { (1.15GHz) 14cores x 3chips and QDR InfiniBand x 2rails; } \\
\text { SUSE Linux Enterprise server } 11\end{array}$ & 73278 & 1192000 & 2490368 & & 2287630 \\
\hline Cray XT5 (Opteron quad core $2.3 \mathrm{GHz}$ ) & 150152 & 1059000 & 4712799 & & 1381400 \\
\hline Cray XE6 (AMD 12-core, 2.1 Ghz w/custom interconnect) & 153408 & 1054000 & 4537344 & & 1288627 \\
\hline IBM BladeCenter cluster of 3060 nodes dual socket $1.8 \mathrm{GHz}$ & 122400 & 1042000 & 2249343 & & 1375776 \\
\hline
\end{tabular}




\section{Computer (Full Precision)}

\begin{tabular}{|c|cccc}
$\begin{array}{c}\text { Number } \\
\text { of Procs } \\
\text { or Cores }\end{array}$ & $\begin{array}{c}\boldsymbol{R}_{\max } \\
\text { GFlop/s }\end{array}$ & $\begin{array}{c}\boldsymbol{N}_{\max } \\
\text { Order }\end{array}$ & $\begin{array}{c}\boldsymbol{N}_{1 / 2} \\
\text { Order }\end{array}$ & $\begin{array}{c}\boldsymbol{R}_{\text {Peak }} \\
\text { GFlop/s }\end{array}$ \\
\hline & & & & \\
\hline 60000 & 1033110 & 2880000 & 1344000 \\
\hline & & & \\
& & & 1375776 \\
\hline 122400 & 1026000 & 2236927 & 1173000 \\
\hline 112800 & 919100 & 3844936 & 1217700 \\
\hline 39680 & 886400 & 3571200 & 1028851 \\
\hline 98928 & 831750 & 3718960 & 10027000 \\
\hline 294912 & 825500 & 4043519 & 14902700 \\
\hline 294912 & 819600 & 3981311 & \\
\hline & & & &
\end{tabular}

Opteron (dual core) LS21 blades plus 6120 nodes dual socket 3.2 GHz PowerXCell 8i (8 SPU + 1 PPU cores) QS22 blades w/InfiniBand

BM iDataPlex DX360M4 (dual socket - 10 core Ivy Bridge $2.8 \mathrm{GHz}$ ) InfiniBand FDR 14

DOE/NNSA/LANL IBM BladeCenter cluster of 3060 nodes dual socket $1.8 \mathrm{GHz}$ Opteron (dual core) LS21 blades plus 6120 nodes dual socket $3.2 \mathrm{GHz}$ PowerXCell 8i (8 SPU + 1 PPU cores) QS22 blades w/InfiniBand

Cray XT5 (AMD six-core 2.6 GHz Istanbul)
IBM Power 775 (IBM POWER7 $3.836 \mathrm{GHz}$ (155 drawers x
256 cores/drawer)) w/Custom

Cray XT-5 AMD six-core $2.6 \mathrm{GHz}$ Istanbul
IBM Blue Gene/P Soltuion (Quad core $0.85 \mathrm{GHz}$ PowerPC
$450 \mathrm{w} /$ custom)

$450 \mathrm{w} /$ custom)

IBM Blue Gene/P Soltuion (Quad core $0.85 \mathrm{GHz}$ PowerPC

$450 \mathrm{w} /$ custom)

Mole-8.5 256 computing nodes, each node contains: 2 2.267GHz 4-core Xeon 5520, 6 nVidia Tesla C2050 (Fermi) GPU cards, w/QDR Infiniband

National Research Center of Parallel Computer Engineering $\&$ Technology 8704 Propriety nodes, 16 core $(.975 \mathrm{GHz}$ w/InfiniBand QDR)

330 IBM iDatPlex DX360M4 Compute nodes: (2x Intel IvyBridge $2.8 \mathrm{GHz} 10$ core) (2x Nvidia K20x GPUs (660 total)) FDR14

\begin{tabular}{|c|c|c|c|c|}
\hline total)) FDR14 & 15840 & 709700 & 1048320 & 1012440 \\
\hline $\begin{array}{l}\text { 1IBM Blue Gene/Q Power BCQ } 1.6 \mathrm{GHz} \text { ( } 4 \text { racks * } 1024 \\
\text { nodes/rack * } 16 \text { cores/node) w/custom }\end{array}$ & 65536 & 689758 & 2752511 & 838861 \\
\hline $\begin{array}{l}\text { IBM Blue Gene/Q IBM BQC } 1.6 \text { GHz w/ Proprietary Nodes: } \\
4096 \text { Cores/node: } 16\end{array}$ & 65536 & 677104 & 2719743 & 838800 \\
\hline $\begin{array}{l}\text { Lomonosov } 4420 \text { nodes of } 2 \times \text { Intel Xeon } 5570 \text { Nehalem }(4 \\
\text { cores, } 2.93 \mathrm{GHz})+680 \text { nodex of } 2 \times \text { Intel Xeon } 5670 \\
\text { Westmere }(6 \text { cores, } 2.93 \mathrm{GHz})+777 \text { nodes of } 2 \times \text { Intel Xeon } \\
5670 \text { Westmere }(4 \text { cores, } 2.53 \mathrm{GHz}) 2 \times \text { M } 2070 \text { Tesla }\end{array}$ & 71492 & 674100 & 2073599 & 1373000 \\
\hline $\begin{array}{l}\text { SGI Altix ICE } 8200 \mathrm{EX} \text { ( } 92 \text { racks Xeon QC } 3.0 \mathrm{Ghz}+18 \\
\text { racks Xeon } 2.93 \mathrm{Ghz} \text { w/Infiniband) }\end{array}$ & 56320 & 544300 & 2458680 & 673259 \\
\hline $\begin{array}{l}\text { IBM BlueGene/L DD2 Prototype cluster (dual } 0.7 \mathrm{GHz} \\
\text { PowerPC } 440 \mathrm{w} / \text { custom) }\end{array}$ & 212992 & 478200 & 2456063 & 596378 \\
\hline $\begin{array}{l}\text { IBM System Blue Gene/P Solution (Quad core } 0.85 \mathrm{GHz} \\
\text { PowerPC } 450 \mathrm{w} / \text { Custom) }\end{array}$ & 163840 & 450300 & 2580479 & 557056 \\
\hline Cray XT5 (Opteron quad core $2.3 \mathrm{GHz}$ ) & 66000 & 463300 & 2078999 & 607000 \\
\hline $\begin{array}{l}\text { IBM Flex System p } 460 \text { IBM POWER7 } 3.55 \mathrm{GHz} \text { ( } 560 \text { nodes } \\
\text { x } 32 \text { cores per node) Infiniband QDR }\end{array}$ & 17920 & 434800 & 2400000 & 508928 \\
\hline $\begin{array}{l}\text { IBM Blue Gene/P Soltuion (Quad core } 0.85 \mathrm{GHz} \text { PowerPC } \\
450 \text { w/custom) }\end{array}$ & 147456 & 415700 & 2958335 & 501350 \\
\hline $\begin{array}{l}\text { IBM iDataPlex DX360M4 ( } 2 \text { socket } 8 \text { core Sandybridge } 2.6 \\
\text { GHz ) Number of nodes: } 464 \text { CPU cores: } 7,424 \text { (464 nodes x } \\
2 \text { sockets/node x } 8 \text { cores/socket) Accelerator: } 464 \text { Intel Phi } \\
\text { (MIC) - } 1 \text { per node Accelerator cores; } 27,840 \text { (464 Phi x } 60 \\
\text { cores/Phi ) w/Infiniband QDR }\end{array}$ & 35264 & 368455 & 768000 & 623467 \\
\hline $\begin{array}{l}\text { IBM iDataPlex DX360M4 (2 socket } 12 \text { core Ivy Bridge } 2.7 \\
\text { GHz) InfiniBand FDR14 }\end{array}$ & 18144 & 352671 & 2370816 & 391910 \\
\hline T-Platforms T-Blade2 (Intel Xeon X5570 quad core, 2.933 & 35360 & 350100 & 2489344 & 414419 \\
\hline
\end{tabular}




\section{Computer (Full Precision)}

\begin{tabular}{|c|c|c|c|c|}
\hline & cores & & & \\
\hline \multicolumn{5}{|l|}{ Ghz, w/QDR InfiniBand) } \\
\hline $\begin{array}{l}\text { BM iDataPlex DX360M4 (dual socket - } 12 \text { core Ivy Bridge } \\
2.7 \mathrm{GHz} \text { ) InfiniBand FDR14 }\end{array}$ & 18240 & 347647 & 2371200 & 393984 \\
\hline $\begin{array}{l}\text { IBM Blue Gene/Q IBM BQC } 1.6 \text { GHz w/ Proprietary Nodes: } \\
2048 \text { Cores/node: } 16\end{array}$ & 32768 & 339834 & 1949695 & 419400 \\
\hline $\begin{array}{l}\text { IBM NeXtScale nx360 M4 Ivy Bridge } 2.5 \mathrm{GHz} \text { Cores: } \\
16,820 \text { ( } 841 \text { nodes * } 2 \text { sockets * } 10 \text { cores/socket) InfiniBand } \\
\text { FDR }\end{array}$ & 16820 & 326572 & 2000000 & 336400 \\
\hline $\begin{array}{l}\text { IBM NeXtScale nx } 360 \text { M4 Ivy Bridge } 2.5 \mathrm{GHz} \text { ( } 812 \text { nodes * } \\
2 \text { sockets * } 10 \text { cores/socket) InfiniBand FDR }\end{array}$ & 16240 & 323611 & 1800000 & 324800 \\
\hline $\begin{array}{l}\text { Cray XE6 (12Core AMD Opteron } 6174 \text { (Magny-Cours) } 2.2 \\
\text { GHz) }\end{array}$ & 45504 & 295500 & 2472456 & 400430 \\
\hline $\begin{array}{l}\text { IBM iDataPlex DX360M4 Intel Sandybridge } 2.7 \mathrm{GHz} \text { ( } 9216 \\
\text { nodes * } 2 \text { sockets * } 8 \text { cores/socket) w/InfiniBand }\end{array}$ & 147456 & 2877000 & 5038080 & 3185049 \\
\hline $\begin{array}{l}\text { IBM BlueGene/L DD2 Prototype cluster (dual } 0.7 \mathrm{GHz} \\
\text { PowerPC } 440 \text { w/custom) }\end{array}$ & 131072 & 280600 & 1769471 & 367001 \\
\hline HITACHI SR16000/M1 322 nodes (3836MHz) & 10304 & 253000 & 1858560 & 316209 \\
\hline HITACHI SR16000-M1/320 (3830MHz) & 9984 & 243900 & 1576960 & 306389 \\
\hline $\begin{array}{l}\text { IBM iDataPlex DX360M2 Intel Nehalem } 2.53 \mathrm{GHz}(3824 \\
\text { nodes * } 2 \text { sockets/node * } 4 \text { cores/socket) w/InfiniBand }\end{array}$ & 30592 & 261631 & 2526944 & 309591 \\
\hline $\begin{array}{l}\text { IBM iDataPlex DX360M4 }(2 \text { socket Sandybridge } 2.6 \mathrm{GHz} \\
\text { nodes: } 234 \text { CPU cores: } 3,744(234 \text { nodes x } 2 \text { sockets/node x } \\
8 \text { cores/socket) Accelerator: } 234 \text { Intel Phi (MIC) - } 1 \text { per node } \\
\text { Accelerator cores; } 14,040(234 * 60) \text { Internconnet: } \\
\text { Infiniband }\end{array}$ & 17784 & 220031 & 896000 & 302515 \\
\hline $\begin{array}{l}\text { IBM Blue Gene/Q Power BCQ } 1.6 \mathrm{GHz}(1 \text { racks * } 1024 \\
\text { nodes/rack * } 16 \text { cores/node) w/custom }\end{array}$ & 16384 & 172691 & 1376255 & 209715 \\
\hline $\begin{array}{l}320 \text { hybrid nodes (Intel 2-way } 2.267 \mathrm{GHz} 4 \text {-core Xeon } 5520 \\
\text { plus } 6 \text { nVidia Tesla C2050 (Fermi) GPU cards per node) } \\
\text { connected with QDR Mellanox Infiniband }\end{array}$ & 29440 & 207300 & 1113600 & 1012653 \\
\hline Intel (6-core Xeon X5660, $2.8 \mathrm{GHz}, 2$ sockets/node, IB QDR) & 23040 & 192500 & 2255040 & 258000 \\
\hline $\begin{array}{l}\text { IBM Blue Gene/P Solution (Quad core } 0.85 \mathrm{GHz} \text { PowerPC } \\
450 \mathrm{w} / \text { custom) }\end{array}$ & 65536 & 190900 & 2654207 & 222822 \\
\hline $\begin{array}{l}\text { IBM Blue Gene/Q Power BCQ } 1.6 \mathrm{GHz}(1 \text { rack * } 1024 \\
\text { nodes/rack * } 16 \text { cores/node) w/Custom }\end{array}$ & 16384 & 188967 & 1409023 & 209715 \\
\hline IBM Power 775 IBM POWER7 $3.836 \mathrm{GHz}$ w/Custom & 8192 & 185100 & 1433088 & 251396 \\
\hline $\begin{array}{l}\text { Dawning 5000A, AMD } 8347 \text { HE Opteron (quadcore, } 1.9 \mathrm{GHz} \text {, } \\
\text { w/Infiniband, Windows HPC server } 1920 \text { nodes) }\end{array}$ & 30720 & 180600 & 300208 & 233472 \\
\hline $\begin{array}{l}\text { IBM iDataPlex DX360M4 Intel Sandybridge } 2.7 \mathrm{GHz} \\
\text { w/InfiniBand ( } 512 \text { nodes * } 2 \text { sockets * } 8 \text { cores } / \text { socket) }\end{array}$ & 8192 & 176947 & 1198080 & 164800 \\
\hline $\begin{array}{l}\text { IBM Blue Gene/Q IBM BQC } 1.6 \mathrm{GHz} \text { w/Proprietary Nodes: } \\
1024 \text { Cores/node: } 16\end{array}$ & 16384 & 172494 & 1376255 & 209700 \\
\hline $\begin{array}{l}\text { IBM System x iDataPlex ( } 2.53 \mathrm{GHz} \text { Quad Core Intel Xeon } \\
\text { w/GigE) }\end{array}$ & 29920 & 168600 & 2716430 & 302790 \\
\hline $\begin{array}{l}\text { IBM System Blue Gene/P Solution (Quad core } 0.85 \mathrm{GHz} \\
\text { PowerPC } 450 \text { w/ Custom) }\end{array}$ & 65536 & 167300 & 1766399 & 222820 \\
\hline $\begin{array}{l}\text { IBM System x iDataPlex dx360 M3 } 1360 \text { nodes (Intel Xeon } \\
\text { X5670 (Westmere EP) } 2.93 \mathrm{GHz} \text { w/Infiniband 4x QDR } \\
\text { QLogic) }\end{array}$ & 16320 & 168800 & 1958400 & 191270 \\
\hline $\begin{array}{l}\text { IBM System x iDataPlex dx360 M3 (Intel Xeon X5670 } \\
\text { (Westmere EP) } 2.93 \mathrm{GHz} \text { w/Infiniband 4x QDR QLogic) }\end{array}$ & 8160 & 165300 & 1305600 & 191270 \\
\hline
\end{tabular}




\section{Computer (Full Precision)}

\begin{tabular}{|c|c|c|c|c|c|}
\hline $\begin{array}{l}\text { IBM Power } 775 \text { (IBM POWER7 } 3.836 \mathrm{GHz} \text { custom } \\
\text { interconnect) }\end{array}$ & 6912 & 159600 & 1429000 & & 212115 \\
\hline $\begin{array}{l}256 \text { x HP DL165 dual socket } 2.3 \mathrm{GHz} \text { AMD(12 core); } 368 \mathrm{x} \\
\text { HP SL160 dual socket } 2.67 \mathrm{GHz} \text { Opteron (hex core); } 150 \mathrm{x} \\
\text { IBM dx360 dual socket } 2.67 \mathrm{GHz} \text { Opteron (hex core); } 564 \mathrm{x} \\
\text { IBM dx340 dual socket } 2.33 \mathrm{GH} \text { Xeon (quad core); } 376 \mathrm{x} \\
\text { Sun/Oracle X2200 dual socket } 2.3 \mathrm{GHz} \text { Opteron (quad core); } \\
512 \mathrm{x} \text { Dell } 1950 \mathrm{e} \text { dual socket } 2.32 \mathrm{GHz} \text { Xeon (quad core); } \\
2225 \text { nodes w/ Myricom } 10 \mathrm{G} \text { interconnect }\end{array}$ & 20925 & 149900 & 1790200 & & 193900 \\
\hline $\begin{array}{l}256 \text { hybrid nodes (Intel } 2 \text {-way } 2.267 \mathrm{GHz} 4 \text {-core Xeon } 5520 \\
\text { plus } 6 \text { nVidia Tesla C2050 (Fermi) GPU cards per node) } \\
\text { connected with QDR Mellanox Infiniband }\end{array}$ & 23552 & 149700 & 710400 & & 809611 \\
\hline $\begin{array}{l}\text { IBM System Blue Gene/P Solution (Quad core } 0.85 \mathrm{GHz} \\
\text { PowerPC } 450 \mathrm{w} / \text { Custom) }\end{array}$ & 65536 & 145400 & 1757183 & & 222820 \\
\hline $\begin{array}{l}\text { IBM iDataPlex DX360M2 (Intel Westmere } 2.4 \mathrm{GHz} \text { Nodes } \\
256 \text { CPU Cores: } 3072 \text { ( } 256 \text { nodes * } 2 \text { sockets * } 6 \text { cores }) \\
\text { GPU: } 512 \text { nVIDIA M2070 w/InfiniBand })(256 \text { nodes * } 2 \\
\text { sockets * } 6 \text { cores * } 4 \text { fp per cycle })+(512 \text { GPUs * } 515.2 \text { fp per } \\
\text { GPU) }\end{array}$ & 10240 & 142700 & 1159000 & & 293273 \\
\hline $\begin{array}{l}\text { IBM BlueGene/L DD2 Prototype cluster (dual } 0.7 \mathrm{GHz} \\
\text { PowerPC } 440 \mathrm{w} / \text { custom) }\end{array}$ & 65536 & 136800 & 1277951 & & 183500 \\
\hline $\begin{array}{l}1200 \text { IBM System x iDataPlex dx360 M3 (Intel Xeon X5650 } \\
\text { (Westmere EP) 2.66 GHz w/Infiniband QDR QLogic) }\end{array}$ & 14400 & 136300 & 1532160 & & 153216 \\
\hline $\begin{array}{l}\text { HP Cluster Platform } 3000 \text { BL460c (Dual Intel Xeon } 3 \mathrm{GHz} \\
\text { quad core E5365 (Clovertown) w/Infiniband 4X DDR) }\end{array}$ & 14400 & 132800 & 1850000 & 250000 & 172608 \\
\hline Intel (6-core Xeon X5660, $2.8 \mathrm{GHz}, 2$ sockets/node, IB QDR) & 15444 & 131500 & 1894464 & & 173000 \\
\hline Cluster Platform 3000 BL460c, Xeon 53xx 3GHz, Infiniband & 14240 & 129300 & 1750000 & & 170880 \\
\hline SGI Altix ICE 8200, Xeon quad core $3.0 \mathrm{GHz}$ & 14336 & 126900 & 1831872 & & 172032 \\
\hline $\begin{array}{l}\text { IBM BladeCenter cluster ( } 360 \text { nodes dual socket } 1.8 \mathrm{GHz} \\
\text { Opteron (dual core) \& } 720 \text { nodes dual socket } 3.2 \mathrm{GHz} \\
\text { PowerXCell } 8 \mathrm{i} \text { QS22 blades w/InfiniBand) }\end{array}$ & 14400 & 126500 & 805759 & & 161856 \\
\hline
\end{tabular}

USC Cluster (256 x HP SL160 dual socket $2.67 \mathrm{GHz}$ Opteron (hex core) $160 \mathrm{x}$ IBM dx360 dual socket $2.67 \mathrm{GHz}$ Opteron (hex core) 112 x HP SL160 dual socket $2.67 \mathrm{GHz}$ Xeon (hex core) $180 \times$ IBM dx340 dual socket $2.33 \mathrm{GH}$ Xeon (quad core) $384 \times$ IBM dx340 dual socket $2.33 \mathrm{GHz}$ Xeon (quad core) $128 \times$ Sun/Oracle X2200 dual socket $2.3 \mathrm{GHz}$ Opteron (quad core) $512 \times$ Dell 1950e dual socket $2.32 \mathrm{GHz}$ Xeon (quad core) $256 \times$ Sun/Oracle x 2200 dual socket $2.3 \mathrm{GHz}$ Opteron (quad core) w/Myrinet 10G

Cray XT5 (quad core $2.3 \mathrm{GHz}$ Optron)

NEC SX-9/E/1280M160

HITACHI SR16000-M1/176(3830MHz)

\begin{tabular}{l|c|ccc}
\hline IBM Power 575 4.7 GHz (w/IB) & 8064 & 115900 & 1128959 & 151603 \\
\hline IBM Power 775 IBM POWER7 3.836 GHz w/Custom & 4608 & 114800 & 1184256 & 86395 \\
\hline
\end{tabular}

Fujitsu FX1Quadcore SPARC64VII (Quad core 2.52GHz infiniband DDR)

320 node iDataPlex (300 dual socket ( 8 core) Sandybridge plus 20 dual socket Sandybridge nodes w/dua 1 Intel Phi) SandyBridge E5-2670 (2.6 GHz) \& Intel PHI $5110 \mathrm{P}$ w/Mellanox FDR

Number of Procs $\boldsymbol{R}_{\max }$ $N_{\max }$ $N_{1 / 2}$ $\boldsymbol{R}_{\text {Peak }}$ or Cores GFlop/s Order Order GFlop/s 


\section{Computer (Full Precision)}

\begin{tabular}{|c|c|c|c|c|}
\hline $\begin{array}{l}\text { Number } \\
\text { of Procs } \\
\text { or Cores }\end{array}$ & $\begin{array}{c}\boldsymbol{R}_{\max } \\
\text { GFlop/s }\end{array}$ & $\begin{array}{l}N_{\max } \\
\text { Order }\end{array}$ & $\begin{array}{c}N_{1 / 2} \\
\text { Order }\end{array}$ & $\begin{array}{c}R_{\text {Peak }} \\
\text { GFlop/s }\end{array}$ \\
\hline 7424 & 98920 & 638975 & & 148378 \\
\hline 10240 & 106100 & 1535480 & & 122880 \\
\hline 13728 & 102800 & & & 181612 \\
\hline 26569 & 102200 & 1700000 & & 127531 \\
\hline 15104 & 101700 & 1740800 & & 139000 \\
\hline 22592 & 101700 & 2220160 & & 117478 \\
\hline 16128 & 100600 & 1152000 & & 1483778 \\
\hline 6720 & 98240 & 1058399 & & 126336 \\
\hline 6656 & 92980 & 960000 & & 125133 \\
\hline 32768 & 92960 & 1302527 & & 111411 \\
\hline 40960 & 91290 & 983039 & & 114688 \\
\hline 12512 & 91030 & 1630720 & & 120115 \\
\hline 9920 & 89010 & 1778304 & & 119040 \\
\hline 8256 & 87890 & 1188864 & 129024 & 96760 \\
\hline
\end{tabular}

SandyBridge E5-2670 (2.6 GHz)) 18 IBM iDataPlex DX360M4 with 2x Phi Nodes: (2 Intel SandyBridge E5-2670 (2.6 GHz) 2 Intel PHI 5110P) w/ Mellanox CX3 Single-Port FDR HCA

Intel Xenon E5-2650 (8 core, 2 GHz) + Nvidia M2050 + Infiniband (Node $=2$ Socket +3 GPU)

SGI Altix ICE 8200EX (Xeon quad core $3.0 \mathrm{GHz}$ w/infiniband)

Cluster Platform 3000 BL460c, Xeon 53xx 2.66GHz, Infiniband

Cray XT3 Red Storm (AMD Opteron 2.4 GHz w/custom)

T2K Open Supercomputer (Todai Combined Cluster) AMD

Quad Core Opteron $(2.3 \mathrm{GHz}) 4$ sockets per node

Myrinet-10G

Cray XT3 dual-core Optron 2.6 Ghz

HITACHI HA8000-tc/HT225 504nodes (2300MHz)

Intel Xeon

IBM Power $5754.7 \mathrm{GHz}$ (w/IB)

IBM Power $5754.7 \mathrm{GHz}(\mathrm{w} / \mathrm{IB})$

IBM System Blue Gene/P Solution (Quad core $0.85 \mathrm{GHz}$

PowerPC $450 \mathrm{w} /$ Custom)

IBM eServer Blue Gene Solution (2way 0.7 GHz PowerPC $440 \mathrm{w} /$ Custom)

Appro Xtreme-X (Opteron 8-core 2.4GHz QDR infiniband) IBM BladeCenter cluster HS21 (3.0 GHz Quad Core Intel $\mathrm{Xeon} \mathrm{w} / \mathrm{IB}$ )

Fujitsu PRIMERGY RX200S5, X5570 (2.93GHz Infiniband DDR)

TSUBAME Grid cluster and TSUBASA cluster TSUBAME: SunFire X4600 w/(Opteron 880 (2.4GHz) 2 cores x 8sockets; NVidia GT200 (1.44GHz) 30multiprocessors x 1 chip x 2boards; ClearSpeed CSX600 (210MHz) 1 core x 2sockets x 1board) plus SunFire X4600 with ClearSpeed X620 w/( Opteron $880(2.4 \mathrm{GHz})$ 2cores x 8sockets ClearSpeed CSX600 (210MHz) 1 core x 2sockets x 1board) TSUBASA: SunBlade X6250 68 nodes( Xeon E5440 (2.83GHz) 4cores x 2sockets )

IBM Blue Gene/Q Prototype II (IBM BQC $1.6 \mathrm{GHz}$ Nodes:512 Cores/node: $16 \mathrm{w} /$ custom)

\begin{tabular}{|c|c|c|c|c|}
\hline $\begin{array}{l}\text { IBM System Blue Gene/P Solution (Quad core } 0.85 \mathrm{GHz} \\
\text { PowerPC } 450 \mathrm{w} / \text { Custom) }\end{array}$ & 32768 & 84310 & 1302527 & 111411 \\
\hline $\begin{array}{l}\text { BullX Cluster (602 dual socket Intel X5650 2.67 GHz, } 215 \\
\text { dual socket Intel X5550 2.67 GHz, } 16 \text { quad socket Intel } \\
\text { X7560 w/InfiniBand) }\end{array}$ & 9376 & 85900 & 1446480 & 99316 \\
\hline $\begin{array}{l}\text { T2K Open Supercomputer (AMD Opteron quad-core, } 2.3 \\
\mathrm{GHz} \text { ) w/ Myrinet } 10 \mathrm{G}\end{array}$ & 12288 & 82980 & 1433600 & 113000 \\
\hline $\begin{array}{l}\text { IBM BladeCenter cluster HS21 ( } 3.0 \mathrm{GHz} \text { Quad Core Intel } \\
\text { Xeon w/ IB) }\end{array}$ & 9824 & 80940 & 1623744 & 117888 \\
\hline IBM Power 5754.7 GHz (w/ IB) & 6400 & 80320 & 1056000 & 120320 \\
\hline IBM Power 5754.7 GHz (w/ IB) & 6656 & 80000 & 1096000 & 125133 \\
\hline $\begin{array}{l}\text { Dell C6100 } 670 \text { nodes (Intel Xeon CPU X5670 2.93GHz } \\
\text { (12-cores/node) w/Infinaband }\end{array}$ & 8040 & 79800 & 1800000 & 94229 \\
\hline
\end{tabular}




\section{Computer (Full Precision)}

\begin{tabular}{|c|c|c|c|c|}
\hline IBM Power 5754.7 GHz (w/IB) & 5376 & 78680 & 907199 & 101068 \\
\hline $\begin{array}{l}\text { TSUBAME Grid cluster and TSUBASA cluster TSUBAME: } \\
\text { SunFire X4600 w/(Opteron } 880(2.4 \mathrm{GHz}) \text { 2cores x 8sockets; } \\
\text { NVidia GT200 (1.44GHz) 30multiprocessors x 1chip x } \\
\text { 2boards; ClearSpeed CSX600 }(210 \mathrm{MHz}) 1 \text { core x 2sockets x } \\
\text { 1board) plus SunFire X4600 with ClearSpeed X620 } \\
\text { w/( Opteron } 880(2.4 \mathrm{GHz}) 2 \text { cores x 8sockets ClearSpeed } \\
\text { CSX600 (210MHz) 1core x 2sockets x 1board) TSUBASA: } \\
\text { SunBlade X6250 } 68 \text { nodes( Xeon E5440 (2.83GHz) 4cores x } \\
\text { 2sockets ) }\end{array}$ & 30976 & 77480 & 995328 & 161816 \\
\hline $\begin{array}{l}\text { T2K Open Supercomputer (Tsukuba) Appro Xtreme-X3 } \\
\text { (AMD Opteron quad-core } 2.3 \mathrm{GHz} \text { Infiniband } 4 \mathrm{X} \text { x } 4 \text { rail) }\end{array}$ & 10000 & 76460 & 1508000 & 92000 \\
\hline $\begin{array}{l}\text { IBM System x iDataPlex ( } 2.53 \mathrm{GHz} \text { Quad Core Intel Xeon } \\
\text { w/GigE) }\end{array}$ & 13440 & 76030 & 1808000 & 136013 \\
\hline $\begin{array}{l}\text { IBM eServer pSeries p5 } 575 \text { (8-way } 1.9 \mathrm{GHz} \text { POWER } 5 \text { w/HP } \\
\text { Sw Interconnect) }\end{array}$ & 12208 & 75760 & 1383600 & 92781 \\
\hline HITACHI SR16000-XM1/108(3300MHz) & 3456 & 73350 & 1145440 & 91238.4 \\
\hline $\begin{array}{l}\text { SuperMicro Xeon Cluster, E } 54624 \text { core, } 2.8 \mathrm{GHz} \text {, Nvidia } \\
\text { Tesla s } 2050 \mathrm{GPU},(128 \text { nodes; w/2 socket \& } 2 \mathrm{GPU} / \text { node) } \\
\text { w/Infiniband }\end{array}$ & 4608 & 75296 & 685567 & 143300 \\
\hline $\begin{array}{l}\text { USC system ( } 384 \text { Sun x2200 2.3GHz AMD 2356, } 512 \text { Dell } \\
\text { pe1950 2.3GHz Intel, Interconnect 10G Myrinet) }\end{array}$ & 10240 & 72050 & 1285200 & 94208 \\
\hline $\begin{array}{l}\text { IBM BladeCenter PS702 Express (IBM POWER7 } 3.00 \mathrm{GHz} \\
\text { (Intelligent Energy Optimization enabled, up to } 3.30 \mathrm{GHz} \text { ) } \\
\text { w/Infiniband) ( } 245 \text { nodes x } 16 \text { cores/node) }\end{array}$ & 3920 & 72030 & 940800 & 103488 \\
\hline $\begin{array}{l}\text { IBM eServer Blue Gene Solution ( } 0.7 \mathrm{GHz} \text { PowerPC } 440 \\
\text { w/custom interconnect) }\end{array}$ & 32768 & 71900 & 884735 & 91750 \\
\hline IBM Power 775 IBM POWER7 3.836 GHz w/Custom & 2816 & 70760 & 907776 & 86395 \\
\hline $\begin{array}{l}\text { IBM BlueGene/L DD2 Prototype cluster (dual } 0.7 \mathrm{GHz} \\
\text { PowerPC } 440 \mathrm{w} / \text { custom) }\end{array}$ & 32768 & 70720 & 933887 & 91750 \\
\hline $\begin{array}{l}\text { IBM Power } 775 \text { (IBM POWER7 } 3.836 \text { GHz Interconnect: } \\
\text { Custom) }\end{array}$ & 2816 & 68320 & 710000 & 86395 \\
\hline $\begin{array}{l}\text { IBM System x iDataPlex (2.26 GHz Quad Core Intel Xeon } \\
\text { w/InfiniBand) }\end{array}$ & 8000 & 66680 & 1610000 & 72320 \\
\hline $\begin{array}{l}\text { IBM System x iDataPlex ( } 2.26 \mathrm{GHz} \text { Quad Core Intel Xeon } \\
\text { w/InfiniBand) }\end{array}$ & 8000 & 66500 & 1554280 & 72320 \\
\hline $\begin{array}{l}\text { IBM System x iDataPlex ( } 2.26 \mathrm{GHz} \text { Quad Core Intel Xeon } \\
\text { w/InfiniBand) }\end{array}$ & 7992 & 65780 & 1374072 & 72248 \\
\hline Columbia - SGI Altix $1.5 \mathrm{GHz}$, Voltaire Infiniband & 13608 & 66567 & 1478736 & 82944 \\
\hline $\begin{array}{l}\text { IBM BladeCenter HX5 } 205 \text { nodes (Intel Xeon E7-4870 } \\
\text { (Westmere EX) } 2.40 \mathrm{GHz}(10 \text { core) Cores: } 8,000 \text { (200 nodes } \\
* 4 \text { sockets * } 10 \text { cores) w/Infiniband QDR }\end{array}$ & 8000 & 64860 & 1099224 & 78720 \\
\hline $\begin{array}{l}\text { Sun Blade } 6048 \text { (Xeon X5560 quad core } 2.8 \mathrm{GHz} \\
\text { w/Infiniband QDR) }\end{array}$ & 6464 & 64630 & 1405152 & 72397 \\
\hline BladeCenter JS21 Cluster, PPC 970, 2.3GHz, Myrinet & 10000 & 63830 & 1458000 & 92000 \\
\hline $\begin{array}{l}\text { IBM eServer pSeries p5 } 575 \text { (8-way } 1.9 \mathrm{GHz} \text { POWER5 } \\
\text { w/HPsw) }\end{array}$ & 10240 & 63390 & 1280000 & 77824 \\
\hline $\begin{array}{l}\text { IBM BladeCenter JS21 Cluster (PPC 970, 2.3GHz } \\
\text { w/Myrinet) }\end{array}$ & 10000 & 62630 & 1458000 & 92000 \\
\hline $\begin{array}{l}\text { IBM Blue Gene Q Prototype (IBM BQC } 1.6 \mathrm{GHz}, 16 \text { core, } \\
\text { Interconnect Proprietary) }\end{array}$ & 8192 & 65347 & 434175 & 104857 \\
\hline IBM Power 750 Express (POWER7 3.55 GHz (w/ Intelligent & 2720 & 61260 & 1100000 & 83994 \\
\hline
\end{tabular}




\section{Computer \\ (Full Precision)}

Number

of Procs

or Cores

$\boldsymbol{R}_{\max }$

$N_{\max }$

$N_{1 / 2}$

$\boldsymbol{R}_{\text {Peak }}$

Energy Optimization enable up to $3.86 \mathrm{GHz}$ ) w/10G Ethernet

Nodes 854 sockets $* 8$ cores)

252 nodes Dell PE1950 Xeon quad core $2.33 \mathrm{GHz}$ plus 254 nodes Dell PE1950 Xeon quad core $2.33 \mathrm{GHz}$ plus 255 nodes Sun X2200M2x64 Opteron quad core $2.3 \mathrm{GHz}$ plus 254 nodes IBM iDataPlex DX340 quad core Xeon $2.33 \mathrm{GHz}$ w/10G Myrinet

(

IBM eServer pSeries p5 575 (8-way $1.9 \mathrm{GHz}$ POWER5 w/HP Sw Interconnect)

IBM Power $5754.7 \mathrm{GHz}(\mathrm{w} / \mathrm{IB})$

IBM Power $5754.7 \mathrm{GHz}$ (w/IB)

IBM Power 750 Express (POWER7 $3.55 \mathrm{GHz}$ (w/ Intelligent

Energy Optimization enable up to $3.86 \mathrm{GHz}$ ) Nodes 80 w/10G Ethernet)

\begin{tabular}{|c|c|c|c|c|c|}
\hline W/ I0G Ethernet) & 2560 & 58310 & 1050000 & & 19052 \\
\hline $\begin{array}{l}\text { IBM System x iDataPlex (2.53 GHz Quad Core Intel Xeon } \\
\text { w/InfiniBand) }\end{array}$ & 6720 & 56810 & 1256000 & & 68006 \\
\hline $\begin{array}{l}\text { Hitachi SR } 16000 \text { Model } 2 \text { (POWER6 4.7GHz (32way), } \\
\text { InfiniBand Fat Tree Network) }\end{array}$ & 4096 & 56650 & 1100000 & & 77004.8 \\
\hline SGI Altix 4700 (Itanium 1.6 GHz) & 9614 & 56520 & 1583232 & & 61530 \\
\hline $\begin{array}{l}\text { TSUBAME Sun Fire X4600 }(2.4 \mathrm{GHz} \text { Opteron } 880 \text { (648 } \\
\text { nodes } * 8 \text { sockets } * 2 \text { cores })+648 \text { ClearSpeed accelerator } \\
\text { cards } * 2 \text { CSX600 processors }) \text { w/Voltaire Infiniband })\end{array}$ & 11664 & 56430 & 1123200 & & 102560 \\
\hline $\begin{array}{l}\text { IBM Power } 750 \text { Express (POWER7 } 3.55 \mathrm{GHz}(\mathrm{w} / \text { Intelligent } \\
\text { Energy Optimization enable up to } 3.86 \mathrm{GHz}) \text { ) w/10G Ethernet } \\
\text { Nodes } 80\end{array}$ & 2560 & 56200 & 1050000 & & 79052 \\
\hline $\begin{array}{l}\text { Xenon Systems } 128 \text { nodes, Dual quad core Xeon E5462, } 2.8 \\
\text { GHz + NVIDIA Tesla S2050 w/infiniband }\end{array}$ & 114688 & 52550 & 670000 & 335000 & 143308 \\
\hline IBM Power 5754.7 GHz (w/IB) & 3584 & 52810 & 767999 & & 67380 \\
\hline $\begin{array}{l}\text { NASA Project Columbia (20x508proc SGI Altix } 30001.5 \\
\text { GHz Itanium2 w/Infiniband) }\end{array}$ & 10160 & 51870 & 1290240 & & 60960 \\
\hline SGI Altix 4700 (Itanium $1.6 \mathrm{GHz}$ ) & 9108 & 51441 & 1260000 & & 58291 \\
\hline HITACHI SR16000-L2/121(4700MHz) & 3872 & 51210 & 844800 & & 72794 \\
\hline $\begin{array}{l}\text { BM Power } 750 \text { Express ( } 86 \text { nodes } * 4 \text { sockets } * 8 \text { cores) } \\
\text { (POWER7 } 3.55 \mathrm{GHz}(\mathrm{w} / \text { Intelligent Energy Optimization } \\
\text { enable up to } 3.86 \mathrm{GHz}) 10 \mathrm{G} \text { Ethernet) }\end{array}$ & 2752 & 50710 & 1100000 & & 84982 \\
\hline $\begin{array}{l}\text { T2K Open Supercomputer/Kyodai, HX600, Opteron Quad } \\
\text { Core } 2.3 \mathrm{GHz} \text {, InfiniBand Fujitsu }\end{array}$ & 6656 & 50510 & 1223040 & 215000 & 61235 \\
\hline $\begin{array}{l}\text { IBM System x iDataPlex }(2.53 \mathrm{GHz} \text { Quad Core Intel Xeon } \\
\text { w/InfiniBand) }\end{array}$ & 6048 & 49900 & 1192000 & & 61206 \\
\hline IBM Power 5754.7 GHz (w/ IB) & 3296 & 48550 & 950000 & & 61965 \\
\hline IBM Power 5754.7 GHz (w/ IB) & 3520 & 47970 & 796000 & & 66176 \\
\hline $\begin{array}{l}\text { TSUBAME Sun Fire X4600 }(2.6 \mathrm{GHz} \text { Opteron } 885 \text { (16 nodes } \\
* 8 \text { sockets } * 2 \text { cores })+2.4 \mathrm{GHz} \text { Opetron } 880(632 \text { nodes } * 8 \\
\text { sockets } * 2 \text { cores }+360 \text { ClearSpeed accelerator cards } * 2 \\
\text { CSX600 processors) w/Voltaire Infiniband) }\end{array}$ & 11088 & 47380 & 1148160 & & 82125 \\
\hline $\begin{array}{l}\text { T-Platforms T-Blade solution Intel Xeon E5472 (quad core, } \\
\text { 3GHz) w/InfiniBand }\end{array}$ & 5000 & 47170 & 750000 & & 60000 \\
\hline $\begin{array}{l}\text { IBM System Blue Gene/P Solution (Quad core } 0.85 \mathrm{GHz} \\
\text { PowerPC } 450 \text { w/Custom) }\end{array}$ & 16384 & 46830 & 933887 & & 55706 \\
\hline
\end{tabular}




\begin{tabular}{|c|c|c|c|c|c|}
\hline $\begin{array}{c}\text { Computer } \\
\text { (Full Precision) }\end{array}$ & $\begin{array}{l}\text { Number } \\
\text { of Procs } \\
\text { or Cores }\end{array}$ & $\begin{array}{c}\boldsymbol{R}_{\max } \\
\text { GFlop/s }\end{array}$ & $\begin{array}{l}N_{\max } \\
\text { Order }\end{array}$ & $\begin{array}{c}N_{1 / 2} \\
\text { Order }\end{array}$ & $\begin{array}{c}\boldsymbol{R}_{\text {Peak }} \\
\text { GFlop/s }\end{array}$ \\
\hline $\begin{array}{l}\text { Dell PE1955 dual-core Intel } 2.66 \mathrm{Ghz} \text { blade w/2 sockets/node } \\
\text { w/Mellanox Infiniband }\end{array}$ & 5848 & 46730 & 1187200 & & 62220 \\
\hline $\begin{array}{l}\text { IBM BladeCenter cluster HS21 ( } 2.5 \mathrm{GHz} \text { Quad Core Intel } \\
\text { Xeon L5420 w/ IB) }\end{array}$ & 5376 & 46040 & 1113600 & & 53670 \\
\hline $\begin{array}{l}\text { IBM cluster ( } 866 \text { dual socket, } 2.6 \mathrm{GHz} \text { Opteron, } 87 \text { quad } \\
\text { socket, } 2.6 \mathrm{GHz} \text { Opteron, } 627 \text { dual socket, } 2.5 \mathrm{GHz} \text { Shanghai, } \\
8 \text { quad socket, QC } 2.5 \mathrm{GHz} \text { Shanghai, with Infiniband) }\end{array}$ & 9304 & 45730 & 1200000 & & 73072 \\
\hline $\begin{array}{l}\text { IBM System x iDataPlex ( } 2.53 \mathrm{GHz} \text { Quad Core Intel Xeon } \\
\text { w/InfiniBand) }\end{array}$ & 5376 & 45480 & 1124000 & & 54405 \\
\hline $\begin{array}{l}\text { Tsubame Sun Galaxy } 4 \text { ( } 2.6 \mathrm{GHz} \text { Opteron } 885 \text { (16 nodes } * 8 \\
\text { sockets } * 2 \text { cores })+2.4 \mathrm{GHz} \text { Opetron } 880(632 \text { nodes } * 8 \\
\text { sockets } * 2 \text { cores }+360 \text { ClearSpeed accelerator cards }) \\
\text { w/Voltaire Infiniband })\end{array}$ & 10728 & 45200 & 971520 & & 84429 \\
\hline $\begin{array}{l}\text { IBM QPACE Cluster ( } 3.2 \mathrm{GHz} \text { IBM PowerXCell8i with } \\
\text { Custom Interconnect) }\end{array}$ & 4608 & 44500 & 487551 & & 55706 \\
\hline Cray XT3 dual-core Optron $2.6 \mathrm{Ghz}$ & 10404 & 43480 & 1064520 & & 54101 \\
\hline $\begin{array}{l}\text { IBM x } 3455 \text { cluster }(822 \text { nodes dual socket dual core } 2.6 \mathrm{GHz} \\
\text { Opteron \& } 641 \text { nodes dual socket dual core } 2.5 \mathrm{GHz} \text { Shanghai } \\
\text { w/ Infiniband) }\end{array}$ & 8416 & 43460 & 591000 & & 68378 \\
\hline $\begin{array}{l}\text { IBM System Blue Gene/P Solution (Quad core } 0.85 \mathrm{GHz} \\
\text { PowerPC } 450 \text { w/Custom) }\end{array}$ & 16384 & 43160 & 909311 & & 55706 \\
\hline Bull NovaScale 5160, Itanium2 1.6 GHz, Quadrics & 8704 & 42900 & & & 55706 \\
\hline $\begin{array}{l}\text { NASA Project Columbia (16x504proc SGI Altix } 30001.5 \\
\text { GHz Itanium2 w/Infiniband) }\end{array}$ & 8064 & 42707 & 1075200 & & 48384 \\
\hline Dell 1955 (dual-core 2.66 GHz IB: Topspin/PCI-X) & 5168 & 41460 & 1097600 & & 54988 \\
\hline $\begin{array}{l}\text { Dell PowerEdge C6100 cluster ( } 2.66 \text { GHz Six Core Xeon } \\
\text { X5650 w/ IB) }\end{array}$ & 4428 & 40310 & 1100000 & & 47110 \\
\hline $\begin{array}{l}\text { IBM System x iDataPlex ( } 2.53 \mathrm{GHz} \text { Quad Core Intel Xeon } \\
\text { w/InfiniBand) }\end{array}$ & 4704 & 39630 & 1051000 & & 47604 \\
\hline $\begin{array}{l}\text { IBM System x iDataPlex ( } 2.8 \mathrm{GHz} \text { Quad Core Intel Xeon } \\
\text { w/InfiniBand) }\end{array}$ & 4104 & 38990 & 1190000 & & 45965 \\
\hline $\begin{array}{l}\text { IBM System x iDataPlex ( } 2.53 \mathrm{GHz} \text { Quad Core Intel Xeon } \\
\text { w/GigE) }\end{array}$ & 6720 & 38790 & 1256000 & & 68006 \\
\hline $\begin{array}{l}\text { Thunderbird - Dell PowerEdge } 1850 \text { (Pentium } 3.6 \text { GHz, } \\
\text { Infiniband) }\end{array}$ & 8000 & 38270 & 1150000 & & 64512 \\
\hline $\begin{array}{l}\text { Tsubame Sun Galaxy } 4 \text { ( } 2.6 \mathrm{GHz} \text { Opteron } 885 \text { (16 nodes } * 8 \\
\text { sockets } * 2 \text { cores })+2.4 \mathrm{GHz} \text { Opetron } 880 \text { ( } 632 \text { nodes } * 8 \\
\text { sockets } * 2 \text { cores }) \text { w/Voltaire Infiniband) }\end{array}$ & 10368 & 38180 & 1334160 & & 49869 \\
\hline $\begin{array}{l}\text { IBM eServer Blue Gene Solution ( } 2 \text { way } 0.7 \mathrm{GHz} \text { PowerPC } \\
440 \text { w/custom interconnect) }\end{array}$ & 16384 & 37330 & 663551 & & 45875 \\
\hline $\begin{array}{l}\text { IBM Power } 750 \text { Express (POWER7 } 3.55 \mathrm{GHz} \text { w/ Intelligent } \\
\text { Energy Optimization enable up to } 3.86 \mathrm{GHz})(47 \text { nodes } * 4 \\
\text { sockets } * 8 \text { cores) Interconnect: Infiniband DDR }\end{array}$ & 1504 & 36880 & 1100416 & & 46443 \\
\hline $\begin{array}{l}\text { IBM eServer BlueGene/L Solution (2 way } 0.7 \mathrm{GHz} \\
\text { PowerPC440 w/Custom interconnect) }\end{array}$ & 16384 & 36490 & 688127 & & 45875.2 \\
\hline Cray XT3 Red Storm (AMD Opteron 2.4 GHz w/custom) & 10848 & 36190 & 1100000 & & 43392 \\
\hline $\begin{array}{l}\text { IBM BlueGene/L DD2 Prototype cluster (2way } 0.7 \mathrm{GHz} \\
\text { PowerPC } 440 \text { w/custom interconnect) }\end{array}$ & 16384 & 36010 & 655359 & & 45875 \\
\hline Earth Simulator $* * * *$ & 5120 & 35860 & 1075200 & 266240 & 40960 \\
\hline $\begin{array}{l}\text { Dell PowerEdge } 1950 \text { (Intel Dural 2.33GHz Quad-core } \\
\text { w/Infiniband) }\end{array}$ & 5408 & 34780 & 761392 & 30000 & 50402 \\
\hline IBM System x iDataPlex (2.53 GHz Quad Core Intel Xeon & 4032 & 32980 & 974000 & & 40804 \\
\hline
\end{tabular}




\begin{tabular}{|c|c|c|c|c|c|}
\hline $\begin{array}{c}\text { Computer } \\
\text { (Full Precision) }\end{array}$ & $\begin{array}{l}\text { Number } \\
\text { of Procs } \\
\text { or Cores }\end{array}$ & $\begin{array}{c}R_{\max } \\
\text { GFlop/s }\end{array}$ & $\begin{array}{l}N_{\max } \\
\text { Order }\end{array}$ & $\begin{array}{c}N_{1 / 2} \\
\text { Order }\end{array}$ & $\begin{array}{c}\boldsymbol{R}_{\text {Peak }} \\
\text { GFlop/s }\end{array}$ \\
\hline \multicolumn{6}{|l|}{ w/InfiniBand) } \\
\hline $\begin{array}{l}\text { IBM QPACE Cluster ( } 3.2 \mathrm{GHz} \text { IBM PowerXCell8i with } \\
\text { Custom Interconnect) }\end{array}$ & 3456 & 32850 & 421631 & & 41779 \\
\hline $\begin{array}{l}\text { Dell PowerEdge M600 (Intel quad core } 2.33 \mathrm{GHz} \text { ), } \\
\text { w/Infiniband }\end{array}$ & 4032 & 31800 & 1309280 & 158280 & 37578 \\
\hline $\begin{array}{l}\text { IBM BladeCenter HS22 cluster (2.66 GHz Quad Core Intel } \\
\text { Xeon w/InfiniBand) }\end{array}$ & 7992 & 31310 & 752640 & & 34048 \\
\hline $\begin{array}{l}\text { IBM System x iDataPlex ( } 2.8 \mathrm{GHz} 6 \mathrm{C} \text { Intel Westmere } \\
\text { w/InfiniBand) }\end{array}$ & 3072 & 30130 & 774144 & & 34406 \\
\hline $\begin{array}{l}\text { IBM System x iDataPlex ( } 2.53 \mathrm{GHz} \text { Quad Core Intel Xeon } \\
\text { w/InfiniBand) }\end{array}$ & 3360 & 28360 & 888000 & & 34003 \\
\hline $\begin{array}{l}\text { IBM eServer Blade Center JS20+ (2-way PowerPC970 } \\
\text { 2.2Ghz w/Myrinet) }\end{array}$ & 4800 & 27910 & 977816 & & 42144 \\
\hline Fujitsu RX200 S5 socket quad core Intel $2.266 \mathrm{GHz} 10 \mathrm{GbE}$ & 6000 & 27777 & 1966080 & & 54000 \\
\hline HP BL460c (Intel Xeon $3 \mathrm{GHz}$ Quad core w/GigE) & 5184 & 27720 & 1537920 & & 62208 \\
\hline $\begin{array}{l}\text { IBM eServer Blue Gene Solution (PowerPC } 4400.7 \mathrm{GHz} \\
\text { w/Custom) }\end{array}$ & 12288 & 27450 & 516095 & & 34406 \\
\hline Intel (1100 node Woodcrest quad core $3 \mathrm{GHz}$ w/Infiniband) & 4400 & 27210 & 400000 & & 52800 \\
\hline $\begin{array}{l}\text { IBM System x iDataPlex ( } 2.8 \mathrm{GHz} \text { Quad Core Intel Nehalem } \\
\text { w/InfiniBand) }\end{array}$ & 2592 & 27140 & 870912 & & 29030 \\
\hline $\begin{array}{l}\text { IBM System x iDataPlex ( } 2.8 \mathrm{GHz} \text { Quad Core Intel Nehalem } \\
\text { w/InfiniBand) }\end{array}$ & 2512 & 26270 & 791280 & & 28134 \\
\hline HP BL460c (Intel Xeon $3 \mathrm{GHz}$ Quad core w/GigE) & 4000 & 25530 & 1351040 & & 48000 \\
\hline $\begin{array}{l}\text { IBM BladeCenter cluster HS21 (2.66GHz Quad Core Intel } \\
\text { Xeon w/GigE) }\end{array}$ & 5040 & 24670 & 781600 & & 53626 \\
\hline $\begin{array}{l}\text { SGI Altix } 4700 \text { (Intel Itanium2 dual-core } 1.6 \mathrm{GHz} \text { w/SGI } \\
\text { NUMAlink) }\end{array}$ & 4096 & 23817 & 881664 & & 26214 \\
\hline IBM Power 5754.7 GHz (w/ IB) & 1536 & 23470 & 768000 & & 28876.8 \\
\hline IBM Power 595 p6 $5.0 \mathrm{GHz}$ w/ InfiniBand & 1536 & 23370 & 684000 & & 30720 \\
\hline $\begin{array}{l}\text { IBM System Blue Gene/P Solution (Quad core } 0.85 \mathrm{GHz} \\
\text { PowerPC } 450 \text { w/Custom) }\end{array}$ & 8192 & 23270 & 651263 & & 27853 \\
\hline $\begin{array}{l}\text { System G } 324 \text { Mac Pro towers, dual quad core } 2.8 \mathrm{GHz} \text { Xeon } \\
\text { w/Infiniband }\end{array}$ & 2520 & 22320 & 545000 & & 28224 \\
\hline $\begin{array}{l}\text { IBM System Blue Gene/P Solution (Quad core } 0.85 \mathrm{GHz} \\
\text { PowerPC } 450 \mathrm{w} / \text { Custom) }\end{array}$ & 8192 & 21910 & 602111 & & 27853 \\
\hline $\begin{array}{l}\text { Sun Constellation (quad core Intel Xeon X5570 2.93Ghz IB } \\
\text { Mellanox) }\end{array}$ & 2144 & 21330 & 551712 & & 25128 \\
\hline $\begin{array}{l}\text { T2K Open Supercomputer (Todai) AMD Opteron } 8356 \text { (quad } \\
\text { core, } 2.3 \mathrm{GHz} \text { ) }\end{array}$ & 4096 & 21090 & 400000 & & 37683 \\
\hline $\begin{array}{l}\text { IBM System Blue Gene/P Solution (Quad core } 0.85 \mathrm{GHz} \\
\text { PowerPC } 450 \text { w/Custom) }\end{array}$ & 8192 & 20860 & 602111 & & 27850 \\
\hline $\begin{array}{l}\text { IBM eServer Blade Center JS20+ (2-way PowerPC } 970 \\
\text { 2.2Ghz w/Myrinet) }\end{array}$ & 3564 & 20530 & 812592 & 180576 & 31363 \\
\hline Cray XT3, (AMD Opteron $2.4 \mathrm{GHz}$ w/custom) & 5200 & 20527 & & & 24960 \\
\hline IBM System p p575 1.9GHz (w/HPS) & 3072 & 20070 & 700000 & & 23347 \\
\hline $\begin{array}{l}\text { Intel Itanium2 Tiger4 (4-way) 1.4GHz Itanium2 w/Quadrics } \\
\text { Elan4 (QsNetII) }\end{array}$ & 4096 & 19940 & 975000 & 110000 & 22938 \\
\hline $\begin{array}{l}\text { IBM BladeCenter cluster HS21 ( } 3.0 \mathrm{GHz} \text { Dual Core Xeon } \\
5160 \mathrm{w} / \mathrm{IB})\end{array}$ & 2080 & 19910 & 968000 & & 24960 \\
\hline $\begin{array}{l}\text { IBM System x iDataPlex ( } 2.53 \mathrm{GHz} \text { Quad Core Intel Xeon } \\
\text { w/GigE) }\end{array}$ & 3360 & 19580 & 900000 & & 34003 \\
\hline
\end{tabular}




\begin{tabular}{|c|c|c|c|c|c|}
\hline $\begin{array}{c}\text { Computer } \\
\text { (Full Precision) }\end{array}$ & $\begin{array}{l}\text { Number } \\
\text { of Procs } \\
\text { or Cores }\end{array}$ & $\begin{array}{c}\boldsymbol{R}_{\max } \\
\text { GFlop/s }\end{array}$ & $\begin{array}{l}N_{\max } \\
\text { Order }\end{array}$ & $\begin{array}{c}N_{1 / 2} \\
\text { Order }\end{array}$ & $\begin{array}{c}\boldsymbol{R}_{\text {Peak }} \\
\text { GFlop/s }\end{array}$ \\
\hline $\begin{array}{l}\text { NASA Project Columbia (8x512proc SGI Altix } 30001.5 \mathrm{GHz} \\
\text { Itanium } 2 \mathrm{w} / \text { Infiniband) }\end{array}$ & 4032 & 19564 & 800000 & & 24192 \\
\hline $\begin{array}{l}\text { IBM BladeCenter cluster HS21 (3.0GHz Dual Core Xeon } \\
5160 \mathrm{w} / \mathrm{IB})\end{array}$ & 2072 & 19550 & 900000 & & 24860 \\
\hline $\begin{array}{l}\text { Intel Itanium2 Tiger4 (4-way) 1.4GHz Itanium2 w/Quadrics } \\
\text { Elan4 (QsNetII) }\end{array}$ & 4032 & 19470 & 960000 & 110000 & 22579 \\
\hline $\begin{array}{l}\text { IBM BladeCenter cluster HS21 (3.0GHz Dual Core Xeon } \\
5160 \mathrm{w} / \mathrm{IB})\end{array}$ & 2072 & 19390 & 844000 & & 24860 \\
\hline $\begin{array}{l}\text { IBM BladeCenter cluster HS21 (3.0GHz Dual Core Xeon } \\
5160 \mathrm{w} / \mathrm{IB})\end{array}$ & 2064 & 18730 & 660000 & & 24770 \\
\hline $\begin{array}{l}\text { IBM BladeCenter cluster HS21 (2.33 GHz Xeon } 5140 \text { w/ } \\
\text { GigE) }\end{array}$ & 3840 & 18600 & 600000 & & 35790 \\
\hline $\begin{array}{l}\text { IBM BladeCenter QS22 cluster (4.0 GHz "Prototype" IBM } \\
\text { PowerXcell 8i w/ IB) }\end{array}$ & 2016 & 18570 & 325375 & 115455 & 30464 \\
\hline $\begin{array}{l}\text { Dell Cluster Intel Pentium Woodcrest ( } 3 \mathrm{GHz} \text { w/Pathscale } \\
\text { Infiniband) }\end{array}$ & 2340 & 18270 & 713000 & & 28080 \\
\hline $\begin{array}{l}\text { IBM eServer Blue Gene Solution (2way } 0.7 \mathrm{GHz} \text { PowerPC } \\
440 \mathrm{w} / \text { Custom) }\end{array}$ & 8192 & 18200 & 442367 & & 22937.6 \\
\hline $\begin{array}{l}\text { IBM eServer Blue Gene Solution, BlueGene/L (IBM } \\
\text { PowerPC } 700 \mathrm{MHz} 440 \times 5 \text { processors w/Proprietary } \\
\text { Interconnect) }\end{array}$ & 8196 & 17810 & 675839 & & 22940 \\
\hline IBM Blue Gene/L (0.7GHz Power PC440 w/ custom) & 8192 & 17730 & 442367 & & 22938 \\
\hline Cray XT3 Opteron dual core $2.6 \mathrm{GHz}$ & 4096 & 17280 & 598672 & & 21420 \\
\hline $\begin{array}{l}\text { SGI Altix XE } 1300 \text { Cluster Solution ( } 512 \text { Xeon } 5355 \\
\text { quad-core } 2.66 \mathrm{GHz} \text { w/Infiniband DDRx ) }\end{array}$ & 2048 & 17250 & 638448 & & 21791 \\
\hline $\begin{array}{l}\text { IBM BladeCenter cluster HS21 (2.33GHz Quad Core Intel } \\
\text { Xeon } 5345 \mathrm{w} / \mathrm{IB})\end{array}$ & 2504 & 17140 & 720000 & & 23337 \\
\hline $\begin{array}{l}\text { IBM System x cluster }(864 \times 3455 \text { nodes and } 86 \text { x } 3755 \text { nodes, } \\
2.6 \mathrm{GHz} \text { Dual Core Opteron w/ IB) }\end{array}$ & 4144 & 17100 & 670000 & & 21549 \\
\hline $\begin{array}{l}\text { IBM System x iDataPlex ( } 2.53 \mathrm{GHz} \text { Quad Core Intel Xeon } \\
\text { w/InfiniBand) }\end{array}$ & 2016 & 17050 & 689000 & & 20402 \\
\hline $\begin{array}{l}\text { Sun Blade } 6048 \text { with X6420 blades (quad core AMD } 2.0 \\
\text { GHz) w/Infiniband }\end{array}$ & 3072 & 16990 & 854784 & & 24576 \\
\hline Cray XT3, (AMD Opteron 2.6 GHz w/custom) & 4096 & 16975 & & & 21299 \\
\hline $\begin{array}{l}\text { Dell Cluster Intel Pentium Woodcrest ( } 3 \mathrm{GHz} \text { w/Pathscale } \\
\text { Infiniband) }\end{array}$ & 2208 & 16570 & 698000 & & 26496 \\
\hline Apple Xserve G5 (IBM PowerPC 970FX 2 GHz w/Myrinet) & 3072 & 16180 & 750000 & 160000 & 24576 \\
\hline Intel (X5550 2.67 GHz dual-quad core w/Infiniband DDR) & 1760 & 15890 & 759296 & 100000 & 18304 \\
\hline HITACHI SR11000-J2/128(2300MHz) & 128 & 15811 & 645120 & 116640 & 18841 \\
\hline $\begin{array}{l}\text { IBM System x iDataPlex ( } 2.5 \mathrm{GHz} \text { Quad Core Intel Xeon w/ } \\
\text { IB) }\end{array}$ & 2048 & 15810 & 642000 & & 20480 \\
\hline $\begin{array}{l}\text { IBM BladeCenter cluster LS20 ( } 2.2 \text { GHz AMD Opteron w/ } \\
\text { GigE) }\end{array}$ & 6400 & 15760 & 840000 & & 28160 \\
\hline Cray X1E (1.13 GHz) & 1020 & 15706 & & & 18442 \\
\hline $\begin{array}{l}\text { IBM BladeCenter QS22 cluster (3.2 GHz IBM PowerXcell 8i } \\
\text { w/ IB) }\end{array}$ & 2016 & 15700 & 325375 & 111615 & 24371 \\
\hline IBM System x cluster (2.6 GHz Dual Core Opteron w/ IB) & 4144 & 15350 & 560000 & & 21550 \\
\hline $\begin{array}{l}\text { IBM BladeCenter cluster HS21 (2.33GHz Quad Core Intel } \\
\text { Xeon } 5345 \text { w/ IB) }\end{array}$ & 2120 & 15290 & 483000 & & 19758 \\
\hline $\begin{array}{l}\text { IBM BladeCenter cluster HS21 ( } 2.33 \mathrm{GHz} \text { Xeon } 5140 \text { w/ } \\
\text { GigE) }\end{array}$ & 3072 & 15160 & 550000 & & 28630 \\
\hline
\end{tabular}




\begin{tabular}{|c|c|c|c|c|c|}
\hline $\begin{array}{c}\text { Computer } \\
\text { (Full Precision) }\end{array}$ & $\begin{array}{l}\text { Number } \\
\text { of Procs } \\
\text { or Cores }\end{array}$ & $\begin{array}{r}\boldsymbol{R}_{\max } \\
\text { GFlop/s }\end{array}$ & $\begin{array}{l}N_{\max } \\
\text { Order }\end{array}$ & $\begin{array}{c}N_{1 / 2} \\
\text { Order }\end{array}$ & $\begin{array}{c}\boldsymbol{R}_{\text {Peak }} \\
\text { GFlop/s }\end{array}$ \\
\hline $\begin{array}{l}\text { Fujitsu PRIMERGY RX200S3 (3.0 GHz Dual Core Xeon } \\
5160 \mathrm{w} / \mathrm{IB})\end{array}$ & 1536 & 15090 & 500000 & 95000 & 18432 \\
\hline $\begin{array}{l}\text { IBM eServer BladeCenter JS21 (4way PowerPC } 970 \\
\text { 2.5GHz w/Myrinet) }\end{array}$ & 2016 & 15040 & 653184 & 145152 & 20160 \\
\hline Cray X1E (1.13 GHz) & 1014 & 14955 & & & 18333 \\
\hline $\begin{array}{l}\text { IBM BladeCenter cluster HS21 (2.66GHz Quad Core Intel } \\
\text { Xeon } 5355 \text { w/ IB) }\end{array}$ & 1792 & 14910 & 588672 & & 19067 \\
\hline Intel Xeon X5550, Quad core $2.67 \mathrm{GHz}$ Infiniband DDR & 1568 & 14710 & $704000^{`}$ & 100000 & 16746 \\
\hline SGI Altix 4700 (Itanium 1.6 GHz) & 2540 & 14593 & 483800 & & 16256 \\
\hline $\begin{array}{l}\text { IBM eServer Blade Center JS20+ (2-way PowerPC970 } \\
\text { 2.195Ghz w/Myrinet) }\end{array}$ & 2520 & 14550 & 670320 & & 22126 \\
\hline Cray XT3 (AMD Opteron 2.4 GHz w/custom) & 3700 & 14170 & 452000 & & 17760 \\
\hline $\begin{array}{l}\text { IBM System x } 3755 \text { cluster }(2.2 \mathrm{GHz} \text { AMD Dual Core } \\
\text { Opteron w/ IB) }\end{array}$ & 4576 & 14070 & 1000000 & & 20130 \\
\hline $\begin{array}{l}\text { IBM BladeCenter QS22 cluster (3.2 GHz IBM PowerXcell 8i } \\
\text { w/ IB) }\end{array}$ & 1800 & 13990 & 309759 & 104063 & 21760 \\
\hline IBM System p p575 (1.9 GHz w/HPS) & 2240 & 13990 & 550000 & & 17024 \\
\hline ASCI Q AlphaServer EV-68(1.25 GHz w/Quadrics) & 8160 & 13880 & 633000 & & 20480 \\
\hline $\begin{array}{l}\text { IBM iDataPlex DX360M2 Westmere }(2.4 \mathrm{GHz} 6 \text { core })+504 \\
\text { nVIDIA M2070 Nodes } 252 \mathrm{w} / \text { InfiniBand }\end{array}$ & 3024 & 137600 & 1150000 & & 288691 \\
\hline $\begin{array}{l}\text { IBM eServer pSeries p5 } 575 \text { (8-way } 1.9 \mathrm{GHz} \text { POWER } 5 \\
\text { w/HPSw) }\end{array}$ & 2048 & 13090 & 806400 & 54000 & 15565 \\
\hline IBM p5 575 (1.5 GHz w/ HPS $)$ & 2560 & 12940 & 600000 & & 15360 \\
\hline $\begin{array}{l}\text { Dell PowerEdge Cluster } 1955 \text { (Intel dual core } 2.67 \mathrm{GHz} \\
\text { w/GigE) }\end{array}$ & 2520 & 12510 & 750000 & & 13459 \\
\hline $\begin{array}{l}\text { Apple XServe platform (1100 dual } 2.3 \mathrm{GHz} \text { IBM PowerPC } \\
970 \text { w/Mellanox Infiniband and Cisco Gigabit Ethernet } \\
\text { secondary fabric) }\end{array}$ & 2200 & 12250 & 620000 & & 20240 \\
\hline $\begin{array}{l}\text { T-Platforms T-Blade solution Intel Xeon E5472 (quad core, } \\
\text { 3GHz) w/InfiniBand }\end{array}$ & 1328 & 12200 & 380000 & & 15936 \\
\hline SGI Altix 4700 (Itanium $1.6 \mathrm{GHz}$ ) 4 & 2024 & 12072 & 655872 & & 12954 \\
\hline $\begin{array}{l}\text { SGI Altix } 4700(1.6 \mathrm{GHz} \text { Itanium2 dual-core w/SGI } \\
\text { NUMAlink4 within nodes and between nodes 4x256) }\end{array}$ & 2016 & 11913 & 440832 & & 13107 \\
\hline $\begin{array}{l}\text { SGI Altix } 3700 \mathrm{Bx} 2(1.6 \mathrm{GHz} \text { Itanium } 2 \text { configured } 16 \times 128 \\
\text { SGI NUMALink) }\end{array}$ & 2016 & 11814 & 494592 & & 12902 \\
\hline $\begin{array}{l}\text { Apple XServe platform (1080 dual } 2.3 \mathrm{GHz} \text { IBM PowerPC } \\
970 \text { w/Mellanox Infiniband and Cisco Gigabit Ethernet } \\
\text { secondary fabric) }\end{array}$ & 2160 & 11770 & 590000 & & 19872 \\
\hline $\begin{array}{l}\text { IBM System Blue Gene/P Solution (Quad core } 0.85 \mathrm{GHz} \\
\text { PowerPC } 450 \text { w/Custom) }\end{array}$ & 4096 & 11710 & 466943 & & 13926 \\
\hline $\begin{array}{l}\text { IBM BlueGene/L DD1 Prototype ( } 0.5 \mathrm{GHz} \text { PowerPC } 440 \\
\text { w/custom) }\end{array}$ & 8192 & 11680 & 331775 & & 16384 \\
\hline SGI Altix 3700 (Itanium Bx2, 1.6 GHz, NUMALink) & 2024 & 11652 & 440832 & & 12954 \\
\hline $\begin{array}{l}\text { SGI Altix } 3700 \mathrm{Bx} 2(1.6 \mathrm{GHz} \text { Itanium } 2 \text { configured } 4 \times 512 \\
\text { SGI NUMALink) }\end{array}$ & 2016 & 11636 & 440832 & & 12902 \\
\hline IBM System p p575 1.9GHz (w/IB) 2 & 1920 & 11470 & 576000 & & 14590 \\
\hline $\begin{array}{l}\text { IBM Blue Gene Solution, BlueGene/P (IBM PowerPC } 850 \\
\text { MHz } 450 \text { processors w/Proprietary Interconnect) }\end{array}$ & 4092 & 11320 & 466943 & & 13500 \\
\hline $\begin{array}{l}\text { IBM BladeCenter cluster HS21 ( } 3.0 \mathrm{GHz} \text { Dual Core Intel } \\
\text { Xeon } 5160 \mathrm{w} / \mathrm{IB} \text { ) }\end{array}$ & 1280 & 11230 & 518400 & & 15360 \\
\hline
\end{tabular}




\begin{tabular}{|c|c|c|c|c|c|}
\hline $\begin{array}{c}\text { Computer } \\
\text { (Full Precision) }\end{array}$ & $\begin{array}{l}\text { Number } \\
\text { of Procs } \\
\text { or Cores }\end{array}$ & $\begin{array}{r}\boldsymbol{R}_{\max } \\
\text { GFlop/s }\end{array}$ & $\begin{array}{l}N_{\max } \\
\text { Order }\end{array}$ & $\begin{array}{c}N_{1 / 2} \\
\text { Order }\end{array}$ & $\begin{array}{c}\boldsymbol{R}_{\text {Peak }} \\
\text { GFlop/s }\end{array}$ \\
\hline $\begin{array}{l}\text { IBM BladeCenter cluster HS21 (3.0GHz Dual Core Xeon } \\
5160 \mathrm{w} / \mathrm{IB})\end{array}$ & 1360 & 11170 & 537200 & 85000 & 16320 \\
\hline $\begin{array}{l}\text { IBM System Blue Gene/P Solution (Quad core } 0.85 \mathrm{GHz} \\
\text { PowerPC } 450 \text { w/Custom) }\end{array}$ & 4096 & 11110 & 466943 & & 13930 \\
\hline $\begin{array}{l}\text { IBM BladeCenter QS22 cluster (3.2 GHz IBM PowerXcell 8i } \\
\text { w/ IB) }\end{array}$ & 1512 & 11110 & 273919 & 94591 & 18278 \\
\hline $\begin{array}{l}\text { Apple XServe platform (1024 dual } 2.3 \mathrm{GHz} \text { IBM PowerPC } \\
970 \text { w/Mellanox Infiniband and Cisco Gigabit Ethernet } \\
\text { secondary fabric) }\end{array}$ & 2048 & 10930 & 520000 & & 18841.6 \\
\hline $\begin{array}{l}\text { Fujitsu PRIMEQUEST580 (1.6GHz Dual Core Itanium2 w/ } \\
\text { IB) }\end{array}$ & 2048 & 10850 & 580000 & 100000 & 13107 \\
\hline $\begin{array}{l}\text { Intel WA Endeavor ( } 285 \text { node Woodcrest 2-dual core } 3 \mathrm{GHz} \\
\text { w/Infiniband) }\end{array}$ & 1140 & 10770 & 512000 & & 13680 \\
\hline $\begin{array}{l}\text { Sun Constellation (quad core Intel Xeon X5570 2.93Ghz IB } \\
\text { Mellanox) }\end{array}$ & 2112 & 10720 & 200000 & & 24752 \\
\hline IBM System p p575 1.9GHz (w/IB) & 1920 & 10610 & 576000 & & 14592 \\
\hline PACS-CS (Hitachi and Fujitsu) Intel Xeon (2.8 GHz w/GigE) & 2560 & 10350 & 722944 & & 14336 \\
\hline IBM eServer pSeries 655 (8-way $1.7 \mathrm{GHz}$ POWER4+) & 2880 & 10310 & 400000 & & 19584 \\
\hline $\begin{array}{l}\text { Apple G5 dual } 2.0 \mathrm{GHz} \text { IBM Power PC } 970 \text { s, w/Infiniband } \\
\text { 4X primary fabric, Cisco Gigabit Ethernet secondary fabric }\end{array}$ & 2200 & 10280 & 520000 & 152000 & 17600 \\
\hline Dell PowerEdge 1750, P4 Xeon $3.06 \mathrm{GHz}$, w/Myrinet & 2500 & 9819 & 630000 & & 15300 \\
\hline BlueGene/L DD2 Prototype (dual PowerPC 4400.7 GHz) & 4096 & 9433 & 479231 & & 11469 \\
\hline BlueGene/L DD2 Prototype (dual PowerPC 4400.7 GHz) & 4096 & 9360 & 331775 & & 11469 \\
\hline $\begin{array}{l}\text { IBM System Cluster } 13502.33 \mathrm{GHz} \text { Intel Xeon } 5345 \\
\text { (w/GigE) }\end{array}$ & 1536 & 9287 & 616000 & & 14315 \\
\hline IBM eServer pSeries 690 (32 way $1.9 \mathrm{GHz}$ POWER4+) & 2176 & 9241 & 370000 & & 16538 \\
\hline Fujitsu PFU RG1000 (1.5GHz Core2Duo w/ GbE) & 2048 & 9045 & 487680 & 180480 & 12288 \\
\hline HITACHI SR11000-K1/80 (2.1 GHz) & 80 & 9036 & 547200 & & 10752 \\
\hline IBM eServer pSeries 690 (32 way 1.9 GHz POWER4+) & 2112 & 8955 & 350000 & & 16051 \\
\hline HITACHI SR11000-K1/80(2.1 GHz) & 80 & 8893 & 489600 & & 10752 \\
\hline $\begin{array}{l}\text { RIKEN Super Combined Cluster(dual Xeon } 3.06 \mathrm{GHz} \\
\text { multiple clusters w/(1x512-1x128-InfiniBand } 4 \mathrm{X} \\
\text { 3x128-Myrinet)GigE secondary) }\end{array}$ & 2048 & 8728 & 474200 & 120000 & 12534 \\
\hline HP RX2600 Itanium $21.5 \mathrm{GHz}$ w/Quadrics & 1936 & 8633 & 835000 & 140000 & 11616 \\
\hline HP 256 Intel Xeon Processor E5472 (3 GHz w/Infiniband) & 1024 & 8616 & 301056 & & 12288 \\
\hline $\begin{array}{l}\text { IBM BladeCenter cluster AMD Opteron LS20 }(2.0 \mathrm{GHz} \\
\text { AMD Opteron w/ GigE) }\end{array}$ & 3920 & 8509 & 660000 & & 15680 \\
\hline SGI Altix 3000 (Itanium $1500 \mathrm{MHz} 4$ clustered w infiniband) & 2016 & 8397 & 600000 & & 12100 \\
\hline $\begin{array}{l}\text { IBM System x } 3550 \text { cluster }(2.66 \mathrm{GHz} \text { Dual Core Intel Xeon } \\
5160 \mathrm{w} / \mathrm{IB})\end{array}$ & 1008 & 8368 & 333000 & & 10725 \\
\hline HP xw8600 workstations Intel X5450@ 3GHz Infiniband 4x & 864 & 8295 & 645000 & & 10368 \\
\hline $\begin{array}{l}\text { IBM System x } 3455 \text { cluster ( } 2.6 \mathrm{GHz} \text { AMD Dual Core } \\
\text { Opteron w/ IB) }\end{array}$ & 2080 & 8210 & 299520 & & 10820 \\
\hline $\begin{array}{l}\text { IBM BladeCenter cluster HS21 ( } 2.66 \mathrm{GHz} \text { Quad-core Intel } \\
\text { Xeon } 5355 \text { w/ IB) }\end{array}$ & 1024 & 8189 & 320000 & & 10895 \\
\hline IBM eServer pSeries 690 (32 way $1.9 \mathrm{GHz}$ POWER4+) & 2048 & 8174 & 360000 & & 15565 \\
\hline Dawning 4000A (quad Opteron 848 2.2Ghz w/Mirinet2000) & 2560 & 8061 & 728480 & 180000 & 11264 \\
\hline AMD Opteron $2 \mathrm{GHz}$, w/Myrinet & 2816 & 8051 & 761160 & 109208 & 11264 \\
\hline Intel Xeon $3.2 \mathrm{GHz}$ w/Myrinet & 1536 & 7737 & 4000000 & & 9830 \\
\hline
\end{tabular}




\begin{tabular}{|c|c|c|c|c|c|}
\hline $\begin{array}{c}\text { Computer } \\
\text { (Full Precision) }\end{array}$ & $\begin{array}{l}\text { Number } \\
\text { of Procs } \\
\text { or Cores }\end{array}$ & $\begin{array}{l}\boldsymbol{R}_{\max } \\
\text { GFlop/s }\end{array}$ & $\begin{array}{l}N_{\max } \\
\text { Order }\end{array}$ & $\begin{array}{c}N_{1 / 2} \\
\text { Order }\end{array}$ & $\begin{array}{c}\boldsymbol{R}_{\text {Peak }} \\
\text { GFlop/s }\end{array}$ \\
\hline ASCI Q AlphaServer EV-68 1.25 GHz w/Quadrics & 4096 & 7727 & 590000 & 126100 & 10240 \\
\hline ASCI Q AlphaServer EV-68 1.25 GHz w/Quadrics & 4096 & 7679 & 576000 & 138600 & 10240 \\
\hline Linux NetworX/Quadrics(2.4 GHz Xeon w/Quadrics) & 2304 & 7634 & 350000 & 75000 & 11059 \\
\hline Intel Xeon x5355 Quad-core (2.66 GHz w/InfiniBand) & 1024 & 7500 & 300000 & & 10895 \\
\hline $\begin{array}{l}\text { IBM eServer pSeries p5 } 575 \text { (16-way } 1.5 \mathrm{GHz} \text { dual core } \\
\text { POWER } 5 \text { w/HP sw) }\end{array}$ & 1536 & 7395 & 400000 & & 9216 \\
\hline IBM SP Power3 416 nodes $375 \mathrm{MHz}$ & 6656 & 7304 & 640000 & & 9984 \\
\hline ASCI White-Pacific, IBM SP Power 3(375 MHz) & 8000 & 7226 & 518096 & 179000 & 12000 \\
\hline $\begin{array}{l}\text { IBM eServer Itanium2 ( } 248 \text { dual } 1.3 \mathrm{GHz} \& 640 \text { dual } 1.5 \\
\text { GHz w/Myrinet) }\end{array}$ & 1776 & 7215 & 540000 & & 10259 \\
\hline $\begin{array}{l}\text { IBM eServer Opteron e } 325 \text { ( } 2 \text { way, } 2.2 \mathrm{GHz} \text { AMD Opteron } \\
\text { w/Myrinet) }\end{array}$ & 2320 & 7185 & 600000 & & 10208 \\
\hline $\begin{array}{l}\text { Dell } 1855 \text { blade system (dual } 3.2 \mathrm{GHz} \text { Intel EM64T, } \\
\text { InfiniBand TopSpin) }\end{array}$ & 1300 & 6989 & 442624 & & 8320 \\
\hline $\begin{array}{l}\text { IBM Power } 795 \text { ( } 4.00 \mathrm{GHz} \text { POWER7, RHEL 6, Intelligent } \\
\text { Energy Optimization enabled, up to } 4.14 \mathrm{GHz})\end{array}$ & 256 & 6902 & 427776 & 22888 & 8487 \\
\hline $\begin{array}{l}\text { Dell PowerEdge SC1425 ( } 2 \text { way Intel Xeon EM64T 3.60GHz } \\
\text { w/Infiniband) }\end{array}$ & 1140 & 6888 & 650000 & & 8208 \\
\hline Fujitsu PRIMEPOWER HPC2500 (2.08GHz) & 1664 & 6860 & 850720 & 118326 & 13844 \\
\hline $\begin{array}{l}\text { Intel WA Endeavor ( } 232 \text { node Woodcrest 2-dual core } 3 \mathrm{GHz} \\
\text { w/Infiniband) }\end{array}$ & 928 & 6855 & 430000 & & 11136 \\
\hline IBM Power 795 (4.00 GHz POWER7, SLES 11 SP1) & 256 & 6830 & 427776 & 22888 & 8487 \\
\hline $\begin{array}{l}\text { IBM eServer pSeries p5 } 575 \text { (16-way } 1.5 \mathrm{GHz} \text { dual core } \\
\text { POWER5 w/High Perf Sw) }\end{array}$ & 1472 & 6748 & 500000 & & 8832 \\
\hline IBM Power 795 ( $4.0 \mathrm{GHz}$ POWER7 ) & 256 & 6653 & 360000 & 58000 & 8486 \\
\hline $\begin{array}{l}\text { Dell PowerEdge SC1425 ( } 2 \text { way Intel Xeon EM64T 3.60GHz } \\
\text { w/Infiniband Topspin) }\end{array}$ & 1152 & 6615 & 600000 & 300000 & 8294 \\
\hline $\begin{array}{l}\text { IBM BladeCenter cluster HS21 ( } 3.0 \mathrm{GHz} \text { Dual Core Intel } \\
\text { Xeon } 5160 \mathrm{w} / \mathrm{GigE} \text { ) }\end{array}$ & 1760 & 6521 & 404800 & & 21120 \\
\hline IBM/Quadrics (2.4 GHz Xeon w/Quadrics QsNet) & 1920 & 6586 & 425000 & 90000 & 9216 \\
\hline IBM eServer pSeries 690 (32 way $1.7 \mathrm{GHz}$ POWER4+) & 1664 & 6363 & 360000 & & 11315 \\
\hline HITACHI SR11000-K2/50(2300MHz) & 50 & 6272 & 450000 & & 7360 \\
\hline $\begin{array}{l}\text { IBM eServer 1350-xSeries } 335 \text { ( } 2 \text { way } 3.06 \mathrm{GHz} \text { Xeon } \\
\text { w/Quadrics) }\end{array}$ & 1456 & 6232 & 400000 & 67000 & 8911 \\
\hline IBM eServer pSeries 690 (32 way 1.7 GHz POWER4+) & 1600 & 6188 & 355000 & & 10880 \\
\hline $\begin{array}{l}\text { IBM eServer Opteron e } 325 \text { (AMD Opteron } 2.0 \mathrm{GHz} \\
\text { w/Myrinet) }\end{array}$ & 2048 & 6155 & 678912 & & 8192 \\
\hline SGI Altix 3700 (Itanium Bx2, 1.6 GHz, NUMALink) & 1012 & 6028 & 440832 & & 6477 \\
\hline $\begin{array}{l}\text { SGI Altix } 4700 \text { (Intel Itanium2 dual-core } 1.6 \mathrm{GHz} \text { w/SGI } \\
\text { NUMAlink) }\end{array}$ & 1024 & 6015 & 423360 & & 6554 \\
\hline $\begin{array}{l}\text { SGI Altix } 3700 \text { (Itanium Bx2, 1.6 GHz, NUMALink, } \\
\text { 10GigEthernet) }\end{array}$ & 1016 & 6007 & 573888 & & 6502 \\
\hline $\begin{array}{l}\text { IBM eServer pSeries p5 } 575 \text { (8-way } 1.9 \mathrm{GHz} \text { POWER } 5 \text { w/HP } \\
\text { Sw Interconnect) }\end{array}$ & 896 & 5917 & 480000 & & 6810 \\
\hline Cray X1 (800 MHz) & 504 & 5895 & 494592 & 53760 & 6451 \\
\hline $\begin{array}{l}\text { IBM eServer pSeries p5 } 575 \text { (8-way } 1.9 \mathrm{GHz} \text { POWER } 5 \text { w/HP } \\
\text { Sw Interconnect) }\end{array}$ & 864 & 5735 & 532800 & & 6566 \\
\hline $\begin{array}{l}\text { IBM eServer Blade Center JS20+ (2-way PowerPC970 } \\
\text { 2.195Ghz w/Myrinet) }\end{array}$ & 1024 & 5659 & 440000 & & 8991 \\
\hline
\end{tabular}




\begin{tabular}{|c|c|c|c|c|c|}
\hline $\begin{array}{c}\text { Computer } \\
\text { (Full Precision) }\end{array}$ & $\begin{array}{l}\text { Number } \\
\text { of Procs } \\
\text { or Cores }\end{array}$ & $\begin{array}{r}R_{\max } \\
\text { GFlop/s }\end{array}$ & $\begin{array}{l}N_{\max } \\
\text { Order }\end{array}$ & $\begin{array}{c}N_{1 / 2} \\
\text { Order }\end{array}$ & $\begin{array}{c}\boldsymbol{R}_{\text {Peak }} \\
\text { GFlop/s }\end{array}$ \\
\hline IBM eServer pSeries 690 (41x32 way $1.7 \mathrm{GHz}$ POWER4+) & 1312 & 5568 & 660000 & 60000 & 8921 \\
\hline $\begin{array}{l}\text { Dell Power Edge } 1855 \text { (2-way Intel Xeon 3.60GHz EM64T } \\
\text { w/GigE) }\end{array}$ & 1260 & 5439 & 200000 & & 9072 \\
\hline Fujitsu PRIMEPOWER HPC2500(1.3GHz) & 2304 & 5406 & 658800 & 100080 & 11980 \\
\hline $\begin{array}{l}\text { MVS-15000BM Cluster IBM JS20 (dual IBM PowerPC } \\
\text { 970FX - 2.2 GHz w/Myrinet) }\end{array}$ & 924 & 5355 & 415800 & 110000 & 8131 \\
\hline Cray X1 (800 MHz) & 441 & 5156 & 451584 & 48384 & 5645 \\
\hline $\begin{array}{l}\text { IBM BladeCenter cluster LS21 (2.6 GHz AMD Opteron } \\
\text { w/Voltaire 4X Infiniband) }\end{array}$ & 1136 & 5005 & 410000 & & 5907 \\
\hline HITACHI SR11000-J1/50 (1.9 GHz) & 50 & 4993 & 396000 & & 6080 \\
\hline $\begin{array}{l}\text { IBM System x } 3550 \text { cluster }(3.0 \mathrm{GHz} \text { Dual Core Intel Xeon } \\
5160 \mathrm{w} / 10 \mathrm{G} \text { Myrinet) }\end{array}$ & 512 & 4919 & 349440 & & 6144 \\
\hline HP RX2600 Itanium 2 (1GHz w/Quadrics) & 1540 & 4881 & 550000 & 110000 & 6160 \\
\hline Cray XT3 (AMD Opterons 2.6 Ghz w/custom) & 1100 & 4782 & 349760 & & 5720 \\
\hline IBM e 326 cluster ( $2.8 \mathrm{GHz}$ AMD Dual Opteron w/ Myrinet) & 1024 & 4754 & 522240 & & 5734 \\
\hline IBM BlueGene/L DD2 Prototype (0.7 GHz PowerPC 440) & 2048 & 4713 & 233471 & & 5734 \\
\hline Cray X1 (800 MHz) & 400 & 4684 & 440320 & 43520 & 5120 \\
\hline $\begin{array}{l}\text { SGI Altix } 4700 \text { (dual-core Itanium2 1.6GHz w/SGI } \\
\text { NUMAlink) }\end{array}$ & 768 & 4603 & 387072 & & 4915 \\
\hline HITACHI SR11000-K1/40 (2.1 GHz) & 40 & 4596 & 446400 & & 5376 \\
\hline Fujitsu PRIMERGY RX200 (Xeon 3.06GHz/Infiniband 4X) & 1024 & 4564 & 485568 & 91584 & 6266 \\
\hline $\begin{array}{l}\text { IBM BladeCenter cluster HS21 ( } 2.33 \mathrm{GHz} \text { Xeon } 5140 \text { w/ } \\
\text { GigE) }\end{array}$ & 768 & 4554 & 280000 & & 7158 \\
\hline Fujitsu PRIMEPOWER HPC2500 (Sparc 1.56GHz) & 1472 & 4552 & 749340 & 90390 & 9185 \\
\hline HP 256 Intel Xeon Processor E5472 (3 GHz w/Gigabit) & 1024 & 4547 & 401408 & & 8640 \\
\hline $\begin{array}{l}\text { IBM System x } 3455 \text { cluster }(2.6 \mathrm{GHz} \text { AMD Dual Core } \\
\text { Opteron w/ IB) }\end{array}$ & 1024 & 4517 & 460000 & & 5325 \\
\hline Compaq AlphaServer SC ES45/EV68 1GHz & 3016 & 4463 & 280000 & 85000 & 6032 \\
\hline $\begin{array}{l}\text { IBM BladeCenter cluster ( } 2.2 \mathrm{GHz} \text { AMD Opteron w/ } \\
\text { Infiniband SDR) }\end{array}$ & 1152 & 4379 & 700000 & & 5069 \\
\hline IBM eServer pSeries 655 (8-way $1.7 \mathrm{GHz}$ POWER4+) & 1152 & 4379 & 450000 & 60000 & 7833.6 \\
\hline $\begin{array}{l}\text { IBM eServer pSeries p5 } 575 \text { (dual core 16-way } 1.5 \mathrm{GHz} \\
\text { POWER5 w/Myrinet) }\end{array}$ & 1024 & 4307 & 515000 & & 6144 \\
\hline Dell PowerEdge 1750 (dual Xeon $3.2 \mathrm{GHz}$ w/Myrinet) & 1020 & 4298 & 420000 & & 6528 \\
\hline Legend DeepComp 6800, Itanium2 $1.3 \mathrm{GHz}$ QsNet & 1024 & 4193 & 491488 & 120000 & 5324.8 \\
\hline $\begin{array}{l}\text { IBM pSeries p690 Turbo ( } 1.3 \mathrm{GHz} 50 \text { servers } / 32 \\
\text { processors/server) } 2 \text { planes Colony switch }\end{array}$ & 1600 & 4184 & 550000 & 93000 & 8320 \\
\hline $\begin{array}{l}\text { Dell } 1855 \text { blade, Intel Irwindale dual } 3.2 \mathrm{GHz} \text { w/InfiniBand } \\
\text { w/MS Windows }\end{array}$ & 896 & 4106 & 440000 & & 5734 \\
\hline $\begin{array}{l}\text { IBM BladeCenter cluster LS21 (2.6 GHz AMD Opteron w/ } \\
\text { Votaire } 4 \text { X Infiniband) }\end{array}$ & 1024 & 4099 & 180000 & & 5325 \\
\hline Compaq AlphaServer SC ES45/EV68 1GHz & 3024 & 4059 & 525000 & 105000 & 6048 \\
\hline Linux NetworX/Quadrics(2.4 GHz Xeon w/Quadrics) & 1900 & 4049 & 350000 & 75000 & 9120 \\
\hline Compaq AlphaServer SC ES45/EV68(1GHz w/Quadrics) & 2560 & 3980 & 360000 & 85000 & 5120 \\
\hline Dell PowerEdge 1750 (dual Xeon 3.2 GHz w/Myrinet) & 992 & 3975 & 300000 & & 6349 \\
\hline $\begin{array}{l}\text { HP Proliant DL140 G3 (dual processor dual core Intel Xeon } \\
\text { 3GHz } 5160 \text { nodes w/Infiniband 4X DDR) }\end{array}$ & 512 & 3859 & 320000 & & 6000 \\
\hline $\begin{array}{l}\text { IBM eServer pSeries p5 } 575 \text { (16-way } 1.5 \mathrm{GHz} \text { dual core } \\
\text { POWER } 5 \text { w/HP sw) }\end{array}$ & 768 & 3851 & 300000 & & 4608 \\
\hline
\end{tabular}




\begin{tabular}{|c|c|c|c|c|c|}
\hline $\begin{array}{c}\text { Computer } \\
\text { (Full Precision) }\end{array}$ & $\begin{array}{l}\text { Number } \\
\text { of Procs } \\
\text { or Cores }\end{array}$ & $\begin{array}{c}\boldsymbol{R}_{\max } \\
\text { GFlop/s }\end{array}$ & $\begin{array}{l}N_{\max } \\
\text { Order }\end{array}$ & $\begin{array}{c}N_{1 / 2} \\
\text { Order }\end{array}$ & $\begin{array}{c}\boldsymbol{R}_{\text {Peak }} \\
\text { GFlop/s }\end{array}$ \\
\hline IBM eServer pSeries 655 (8 way $1.5 \mathrm{GHz}$ POWER4+) & 1024 & 3812 & 428800 & 42400 & 6144 \\
\hline $\begin{array}{l}\text { IBM Power } 795 \text { ( } 4.25 \mathrm{GHz} \text { POWER7, SLES } 11 \text { SP1, } \\
\text { TurboCore mode enabled) }\end{array}$ & 128 & 3784 & 427776 & 20429 & 4358 \\
\hline $\begin{array}{l}\text { EDA Express }(2.4-2.8 \mathrm{GHz} \text { AMD x } 86-64 \text { Opteron - } 816 \text { cores } \\
\text { from IBM plus } 384 \text { cores from HP w/GigE) }\end{array}$ & 1200 & 3782 & 352000 & & 5760 \\
\hline $\begin{array}{l}\text { IBM eServer 1350-xSeries } 335 \text { (dual } 3.06 \mathrm{GHz} \text { Xeon } \\
\text { w/GigE) }\end{array}$ & 1000 & 3755 & 390000 & & 6120 \\
\hline IBM eServer pSeries 655 ( 8 way $1.5 \mathrm{GHz}$ POWER4+) & 1008 & 3686 & 403200 & 40000 & 6048 \\
\hline IBM Power 795 ( 4.25 GHz POWER7 ) & 128 & 3676 & 260000 & 48000 & 4358 \\
\hline $\begin{array}{l}\text { IBM JS21 Blade Center (128 PowerPC } 9702.5 \mathrm{GHz}, 128 \\
\text { nodes 4proc/node w/Myrinet) }\end{array}$ & 512 & 3637 & 331776 & 69632 & 5120 \\
\hline Intel Xeon $3 \mathrm{GHz}$ dual core w/Murinet 2000 & 544 & 3601 & 241684 & & 6528 \\
\hline $\begin{array}{l}\text { IBM Power } 780 \text { ( } 3.7 \mathrm{GHz} \text { POWER } 7+\text {, RHEL } 6.3 \text {, Intelligent } \\
\text { Energy Optimization enabled, up to } 4144 \mathrm{MHz})\end{array}$ & 128 & 3575 & 321451 & 21000 & 4243 \\
\hline Cray X1 (800 MHz) & 300 & 3522 & 376320 & 38400 & 3840 \\
\hline IBM pSeries 690 Turbo $1.3 \mathrm{GHz}$ & 1280 & 3406 & 317000 & & 6656 \\
\hline $\begin{array}{l}\text { IBM eServer pSeries p5 } 575 \text { (8-way } 1.9 \mathrm{GHz} \text { POWER } 5 \text { w/HP } \\
\text { Sw Interconnect) }\end{array}$ & 512 & 3392 & 320000 & & 3891 \\
\hline HPTi Intel Xeon(2.2 Ghz,dual w/Myrinet) & 1536 & 3337 & 285000 & 75000 & 6758 \\
\hline HITACHI SR11000-H1/56 (1700MHz) & 896 & 3310 & 413280 & 52920 & 6093 \\
\hline HITACHI SR11000-H1/50(1700MHz) & 50 & 3295 & 392400 & 49860 & 5440 \\
\hline Dell PowerEdge 1850 (Xeon EM64T 3.2 GHz w/Infiniband) & 896 & 3256 & 148224 & & 5734 \\
\hline IBM $\mathrm{p} 575+32$ nodes $\mathrm{w} / 16$ processor SMP/node & 512 & 3247 & 392000 & & 3891.2 \\
\hline $\begin{array}{l}\text { IBM eServer 1350-xSeries } 335 \text { ( } 2 \text { way } 3.06 \mathrm{GHz} \text { Xeon } \\
\text { w/Myrinet-2000) }\end{array}$ & 768 & 3231 & 301000 & 59000 & 4700 \\
\hline IBM p690 cluster, Power $41.3 \mathrm{GHz}$ & 1200 & 3210 & 300000 & & 6240 \\
\hline $\begin{array}{l}\text { HP CP3000 (576 Intel Xeon Processor X3.6GHz/800-2MB } \\
\text { w/Infiniband) }\end{array}$ & 576 & 3059 & 300000 & & 4082 \\
\hline SGI Altix $3700 \mathrm{Bx} 2 \quad$ (Itanium2, 1.6 GHz 9MB) & 510 & 3073 & 312480 & & 3264 \\
\hline $\begin{array}{l}\text { SGI Altix } 4700 \text { (dual-core Itanium2 processors @ } 1.6 \mathrm{GHz} \\
\text { w/SGI NUMAlink) }\end{array}$ & 512 & 3071 & 311808 & & 3277 \\
\hline IBM eServer pSeries 570 (8 way 1.9 GHz POWER5 w/GigE) & 720 & 3068 & 375500 & & 5472 \\
\hline Dell Cluster Pentium 4 (3.2 GHz, w/GigE) & 860 & 3064 & 460000 & & 5504 \\
\hline $\begin{array}{l}\text { IBM eServer HS20 cluster ( } 2 \text { way } 3.2 \mathrm{GHz} \text { Intel Xeon } \\
\text { EM64T w/GigE) }\end{array}$ & 1000 & 3059 & 326000 & & 6400 \\
\hline IBM SP Power3 208 nodes $375 \mathrm{MHz}$ & 3328 & 3052 & 371712 & & 4992 \\
\hline $\begin{array}{l}\text { HP Cluster (Dual Intel Quad Core Xeon EM64 processor } \\
\text { w/Infiniband) }\end{array}$ & 320 & 2999 & 224128 & & 3943 \\
\hline $\begin{array}{l}\text { HP Cluster (Dual Intel Quad Core Xeon EM64 processor } \\
\text { w/Infiniband) }\end{array}$ & 320 & 2976 & 202144 & & 4044 \\
\hline $\begin{array}{l}\text { IBM eServer Blade Center JS20 (2-way PowerPC970 2.2Ghz } \\
\text { w/Myrinet) }\end{array}$ & 504 & 2948 & 310000 & & 4435.2 \\
\hline Cray X-1 (800 MHz) & 252 & 2932.9 & 338688 & 44288 & 3225.6 \\
\hline Compaq Alphaserver SC ES45/EV68(1GHz w/Quadrics) & 2048 & 2916 & 272000 & & 4096 \\
\hline NEC SX-8/192M24 (24 nodes 8 proc/node) & 192 & 2914 & 431616 & & 3072 \\
\hline HITACHI SR11000-H1/50 (1700MHz) & 800 & 2909 & 396000 & 84600 & 5440 \\
\hline
\end{tabular}




\begin{tabular}{|c|c|c|c|c|c|}
\hline $\begin{array}{c}\text { Computer } \\
\text { (Full Precision) }\end{array}$ & $\begin{array}{l}\text { Number } \\
\text { of Procs } \\
\text { or Cores }\end{array}$ & $\begin{array}{l}\boldsymbol{R}_{\max } \\
\text { GFlop/s }\end{array}$ & $\begin{array}{l}N_{\max } \\
\text { Order }\end{array}$ & $\begin{array}{c}N_{1 / 2} \\
\text { Order }\end{array}$ & $\begin{array}{c}\boldsymbol{R}_{\text {Peak }} \\
\text { GFlop/s }\end{array}$ \\
\hline $\begin{array}{l}\text { SGI Altix } 3700 \mathrm{Bx} 2 \text { (1.5 GHz Itanium2 configured 18x64 } \\
\text { GigE) }\end{array}$ & 1200 & 2887 & 336000 & & 7200 \\
\hline SGI Altix 3700 Bx2 (1.5 GHz Itanium2) & 510 & 2869 & 317520 & & 3060 \\
\hline $\begin{array}{l}\text { Dell } 1855 \text { blade system (dual } 3.2 \mathrm{GHz} \text { Intel Irwindale w/MS, } \\
\text { InfiniBand) }\end{array}$ & 854 & 2864 & 300000 & & 5466 \\
\hline IBM Xeon Cluster $2.4 \mathrm{MHz}$ w/ Myrinet & 1024 & 2847 & 230000 & & 4915 \\
\hline $\begin{array}{l}\text { IBM eServer Blade Center JS20+ (2-way PowerPC970 } \\
\text { 2.195Ghz w/Myrinet) }\end{array}$ & 528 & 2816 & 320000 & & 4635.84 \\
\hline Cray X-1 (800 MHz) & 240 & 2793.2 & 337920 & 43264 & 3072.0 \\
\hline $\begin{array}{l}\text { IBM eServer Opteron e } 326 \text { Cluster ( } 2 \text { way, } 2.4 \mathrm{GHz} \text { AMD } \\
\text { Opteron w/GigE) }\end{array}$ & 920 & 2791 & 417430 & & 4416 \\
\hline $\begin{array}{l}\text { IBM eServer Opteron e } 326 \text { Cluster ( } 2 \text { way, } 2.4 \text { GHz AMD } \\
\text { Opteron w/InfiniBand) }\end{array}$ & 704 & 2724 & 337920 & & 3379 \\
\hline Cray X-1 (800 MHz) & 234 & 2719.0 & 329472 & 44544 & 2995.2 \\
\hline IBM eServer pSeries 690 Turbo(1.3 GHz Power 4) & 1056 & 2713.0 & 240000 & & 5491 \\
\hline Cray X-1 (800 MHz) & 225 & 2614.2 & 330240 & 38400 & 2880.0 \\
\hline $\begin{array}{l}\text { HP Cluster (Dual Intel Quad Core Xeon EM64 processor } \\
\text { w/Infiniband) }\end{array}$ & 320 & 2614 & 129408 & & 3940 \\
\hline Cray X-1 (800 MHz) & 224 & 2609.5 & 329728 & 38912 & 2867.2 \\
\hline $\begin{array}{l}\text { IBM eServer HS20 cluster ( } 2 \text { way } 3.2 \mathrm{GHz} \text { Intel Xeon } \\
\text { EM64T w/GigE) }\end{array}$ & 640 & 2554 & 272009 & & 4096 \\
\hline Dell PowerEdge 1850, Xeon EM64T (3.2 GHz w/Myrinet) & 784 & 2540 & 200000 & & 5017 \\
\hline IBM SP Power3 158 nodes $375 \mathrm{MHz}$ & 2528 & 2526. & 371712 & 102400 & 3792 \\
\hline $\begin{array}{l}\text { IBM Power } 780 \text { ( } 3.44 \mathrm{GHz} \text { POWER7, SLES } 11 \mathrm{SP} 1 \text {, } \\
\text { Intelligent Energy Optimization enabled, up to } 3.780 \mathrm{GHz})\end{array}$ & 96 & 2512 & 310176 & 15508 & 2903 \\
\hline Cray X-1 (800 MHz) & 220 & 2481 & 317440 & & 2816 \\
\hline Intel dual Pentium Xeon (3.06 GHz w/Myrinet) & 598 & 2455 & 252000 & & 3660 \\
\hline SGI Altix 3000 (1500 MHz Itanium 2) & 510 & 2439 & 252960 & & 3060 \\
\hline Cray X-1 (800 MHz) & 208 & 2416.5 & 292864 & 38912 & 2662.4 \\
\hline ASCI Red Intel Pentium II Xeon core $333 \mathrm{MHz}$ & 9632 & 2379.6 & 362880 & 75400 & 3207 \\
\hline Cray X-1 (800 MHz) & 252 & 2368 & 135555 & & 3226 \\
\hline SGI Altix 3000 (1.3 GHz Itanium2) & 496 & 2338 & 193536 & & 2579 \\
\hline IBM p690 cluster, Power $41.3 \mathrm{GHz}$ & 864 & 2310 & 275000 & 62000 & 4493 \\
\hline $\begin{array}{l}\text { Dell Power Edge } 1855 \text { (2-way Intel Xeon 3.60GHz EM64T } \\
\text { w/Infiniband) }\end{array}$ & 420 & 2303 & 529200 & & 3024 \\
\hline Cray X-1 (800 MHz) & 196 & 2276.4 & 301056 & 34304 & 2508.8 \\
\hline $\begin{array}{l}\text { IBM BlueGene/L DD2 Prototype ( } 0.7 \text { GHz PowerPC } 440 \\
\text { w/custom) }\end{array}$ & 1024 & 2220 & 172031 & & 2867 \\
\hline Atipa Tech. Pentium 4 (1.8 GHz w/Myrinet) & 1024 & 2207. & 280000 & 56000 & 3686 \\
\hline NEC SX-6/248M31(typeE) (1.77ns) & 248 & 2155 & 220224 & 22816 & 2894.16 \\
\hline ASCI Blue-Pacific SST, IBM SP 604E(332 MHz) & 5808 & 2144. & 431344 & 432344 & 3868 \\
\hline $\begin{array}{l}\text { Dell PE1 } 855 \text { blade (dual 3.2GHz Intel 64-bit Xeons w/ } \\
\text { Topspin Infiniband) }\end{array}$ & 400 & 2141 & 290560 & & 2560 \\
\hline ASCI Red Intel Pentium II Xeon core $333 \mathrm{MHz}$ & 9472 & 2121.3 & 251904 & 66000 & 3154 \\
\hline Apple Xserve G5 (dual 2.0 GHz w/Myrinet) & 448 & 2104 & 221376 & 64000 & 3584 \\
\hline Cray X-1 (800 MHz) & 180 & 2099.9 & 291840 & 34816 & 2304.0 \\
\hline Compaq Alphaserver SC ES45/EV68 1GHz & 1520 & 2096 & 390000 & 71000 & 3040 \\
\hline
\end{tabular}




\begin{tabular}{|c|c|c|c|c|c|}
\hline $\begin{array}{c}\text { Computer } \\
\text { (Full Precision) }\end{array}$ & $\begin{array}{l}\text { Number } \\
\text { of Procs } \\
\text { or Cores }\end{array}$ & $\begin{array}{c}\boldsymbol{R}_{\max } \\
\text { GFlop/s }\end{array}$ & $\begin{array}{l}N_{\max } \\
\text { Order }\end{array}$ & $\begin{array}{c}N_{1 / 2} \\
\text { Order }\end{array}$ & $\begin{array}{c}\boldsymbol{R}_{\text {Peak }} \\
\text { GFlop/s }\end{array}$ \\
\hline IBM eServer Intel Tiger4 (quad 1.3GHz Itanium2 w/Myrinet) & 512 & 2082 & 490160 & & 2662 \\
\hline Apple Xserve G5 (dual 2 GHz IBM Power PC 970) & 448 & 2073 & 220032 & & 3584 \\
\hline Intel (dual-core Xeon $3.2 \mathrm{GHz}$ w/IB switch) & 448 & 2060 & 230000 & & 2867 \\
\hline NEC SX-8R/64M8 & 64 & 2056 & 352256 & & 2253 \\
\hline Compaq Alphaserver SC ES45/EV68 1.25GHz & 1024 & 2037 & 320000 & & 2560 \\
\hline Fujitsu M9000 (SPARC64 VII 2.52GHz, quad core) & 256 & 2032.0 & 268128 & 46000 & 2580.48 \\
\hline Sun M9000 (SPARC64 VII 2.52GHz, quad core) & 256 & 2032.0 & 268128 & 46000 & 2580.48 \\
\hline Self-made AMD Opteron dual (2.2GHz w/Infiniband) & 576 & 2028 & 274000 & 24950 & 2534 \\
\hline IBM eServer Cluster HS20 (2.8 GHz Xeon w/GigE) & 610 & 2026 & 340600 & & 3416 \\
\hline $\begin{array}{l}\text { HP-DL580-G5 (Intel Xeon (Tigerton) } 2.933 \mathrm{GHz} \text { quad core } \\
\text { quad processor X7350 nodes w/10Gbps PARAMNet-3) }\end{array}$ & 256 & 2013 & 351232 & & 3000 \\
\hline $\begin{array}{l}\text { IBM eServer HS20 cluster ( } 2 \text { way } 3.2 \mathrm{GHz} \text { Intel Xeon EM64T } \\
\text { w/GigE) }\end{array}$ & 640 & 2010 & 272009 & & 4096 \\
\hline PowerEdge HPC Cluster (2.4 GHz Xeon w/GigE) & 600 & 2004. & 253400 & 42200 & 2880 \\
\hline IBM p690 cluster, Power $41.3 \mathrm{GHz}$ & 768 & 2002 & 252000 & & 3994 \\
\hline Self made (256 nodes dual 3.06GHz Intel Xeon w/ GigE) & 512 & 1997 & 331968 & & 3133 \\
\hline Linux Networx (dual 3.06GHz Intel Xeon processor w/GigE) & 512 & 1997 & 331968 & & 3133 \\
\hline $\begin{array}{l}\text { Fujitsu-Siemens hpcLine (Xeon "Nocona" 64-bit 3.2GHz } \\
\text { w/InfiniBand) }\end{array}$ & 400 & 1978 & 220000 & 3200 & 2560 \\
\hline IBM Power 575 (4.7 GHz POWER6 SLES 10 SP2) & 128 & 1975 & 200000 & 22500 & 2406.4 \\
\hline Cray X-1 (800 MHz) & 169 & 1965.9 & 281216 & 31616 & 2163.2 \\
\hline $\begin{array}{l}\text { BM Power } 770 \text { ( } 3.8 \mathrm{GHz} \text { POWER } 7+\text {, RHEL } 6.3 \text {, Intelligent } \\
\text { Energy Optimization enabled, up to } 4312 \mathrm{MHz})\end{array}$ & 64 & 1948 & 336384 & 14750 & 2208 \\
\hline IBM AMD Opteron (2.2 GHz w/Infiniband) & 600 & 1930 & 400000 & & 2640 \\
\hline Apple Xserve G5 (dual 2 GHz IBM Power PC 970) & 440 & 1911 & 208896 & & 3520 \\
\hline SGI Altix 3000 (Itanium 2, $1.3 \mathrm{GHz}$ ) & 416 & 1793 & 298799 & 298799 & 2163 \\
\hline IBM SP 112 nodes (375 MHz POWER3 High) & 1792 & 1791 & 275000 & 275000 & 2688 \\
\hline $\begin{array}{l}\text { IBM Power } 780 \text { ( } 3.86 \mathrm{GHz} \text { POWER7, RHEL 6, Intelligent } \\
\text { Energy Optimization enabled, up to } 3.94 \mathrm{GHz})\end{array}$ & 64 & 1772 & 224256 & 11700 & 2021 \\
\hline $\begin{array}{l}\text { IBM eServer Cluster 1350-xSeries } 3352.8 \mathrm{GHz} \text { Xeon } \\
\text { w/Myrinet }\end{array}$ & 512 & 1762 & 240000 & 37000 & 2867 \\
\hline IBM p690+ POWER4+ (1.7 GHz w-plane SP Switch2) & 544 & 1760 & 400000 & & 3699 \\
\hline IBM xSeries x335 (dual 2.4 GHz Intel Xeon w/GigE) & 1024 & 1755 & 335000 & 41600 & 4915 \\
\hline IBM eServer pSeries 690 (32 way 1.9 GHz POWER4+) & 352 & 1714 & 372000 & & 2675.2 \\
\hline HITACHI SR8000/MPP/1152(450MHz) & 1152 & 1709.1 & 141000 & 16000 & 2074 \\
\hline IBM eServer pSeries 690 Turbo(1.3 GHz Power 4) & 624 & 1696.0 & 221000 & & 3245 \\
\hline Cray X-1 (800 MHz) & 144 & 1676.9 & 258048 & 29184 & 1843.2 \\
\hline IBM xSeries Cluster Dual Xeon $3.06 \mathrm{GHz}$ w/Myrinet & 486 & 1667 & 213120 & & 2974 \\
\hline HITACHI SR8000-F1/168(375MHz) & 168 & 1653. & 160000 & 19560 & 2016 \\
\hline $\begin{array}{l}\text { IBM eServer Intel Tiger4 (4 way } 1.3 \mathrm{GHz} \text { Itanium } 2 \\
\text { w/Myrinet) }\end{array}$ & 512 & 1636 & 430079 & & 2662 \\
\hline $\begin{array}{l}\text { IBM eServer pSeries } 655 \text { ( } 8 \text { way } 1.7 \text { GHz POWER4+ } \\
\text { w/GigE) }\end{array}$ & 544 & 1636 & 335000 & & 3699.2 \\
\hline ASCI Red Intel Pentium II Xeon core 333Mhz & 6720 & 1633.3 & 306720 & 52500 & 2238 \\
\hline IBM eServer Intel Tiger4 (4 way $1.3 \mathrm{GHz}$ Itanium2 & 500 & 1616 & 419999 & & 2600 \\
\hline
\end{tabular}




\begin{tabular}{|c|c|c|c|c|c|}
\hline $\begin{array}{c}\text { Computer } \\
\text { (Full Precision) }\end{array}$ & $\begin{array}{l}\text { Number } \\
\text { of Procs } \\
\text { or Cores }\end{array}$ & $\begin{array}{c}\boldsymbol{R}_{\max } \\
\text { GFlop/s }\end{array}$ & $\begin{array}{l}N_{\max } \\
\text { Order }\end{array}$ & $\begin{array}{c}N_{1 / 2} \\
\text { Order }\end{array}$ & $\begin{array}{c}\boldsymbol{R}_{\text {Peak }} \\
\text { GFlop/s }\end{array}$ \\
\hline \multicolumn{6}{|l|}{ w/Myrinet) } \\
\hline SGI ASCI Blue Mountain & 5040 & 1608. & 374400 & 138000 & 2520 \\
\hline IBM eServer Cluster HS20 (2.8 GHz Xeon w/GigE) & 480 & 1602 & 275600 & & 2688 \\
\hline $\begin{array}{l}\text { Dell PowerEdge 1850s (dual } 3.2 \mathrm{GHz} \text { Intel EM64T } \\
\text { w/Myrinet) }\end{array}$ & 400 & 1578 & 190000 & & 2560 \\
\hline IBM eServer (Opteron $2.2 \mathrm{GHz}$ w/Infiniband) & 576 & 1575 & 200000 & & 2534.4 \\
\hline NEC SX-6/192M24 & 192 & 1484 & 200064 & 16128 & 1536 \\
\hline IBM eServer pSeries 655 (4 way $1.7 \mathrm{GHz}$ POWER4+) & 384 & 1477 & 174000 & 40000 & 2611 \\
\hline Cray X-1 (800 MHz) & 126 & 1473.3 & 241920 & 30080 & 1612.8 \\
\hline IBM eServer (Opteron $2.2 \mathrm{GHz}$ w/Infiniband) & 484 & 1447 & 200000 & & 2129.6 \\
\hline $\begin{array}{l}\text { IBM eServer pSeries } 690 \text { Turbo(1.3 GHz Power } 4 \\
\text { w/Federation) }\end{array}$ & 512 & 1456.0 & 307200 & & 2662 \\
\hline $\begin{array}{l}\text { IBM BlueGene/L Test Prototype, PowerPC } 440500 \mathrm{MHz} \\
\text { (custom processor/interconnect) }\end{array}$ & 1024 & 1435 & 98304 & & 2048 \\
\hline IBM eServer pSeries 690 Turbo (1.7 GHz POWER4+) & 384 & 1424 & 325000 & & 2611.2 \\
\hline IBM SP 328 nodes (375 MHz POWER3 Thin) & 1312 & 1417. & 374000 & 374000 & 1968 \\
\hline Cray X-1 (800 MHz) & 121 & 1411.1 & 239360 & 26240 & 1548.8 \\
\hline SGI Altix 3700 (1.5 GHz Itanium2) & 255 & 1405 & 211680 & & 1530 \\
\hline $\begin{array}{l}\text { MVS-5000BM Cluster IBM JS20 (dual IBM PowerPC } 970 \text { - } \\
\text { 1.6 GHz w/Myrinet) }\end{array}$ & 330 & 1401 & 280000 & 45000 & 2112 \\
\hline Cray X-1 (800 MHz) & 120 & 1400.4 & 230400 & 26496 & 1536.0 \\
\hline IBM xSeries 335 cluster (dual 3.06GHz Xeon w/InfiniBand) & 384 & 1389 & 120000 & & 2350 \\
\hline IBM eServer pSeries 690 Turbo(1.3 GHz Power 4 w/Colony) & 512 & 1384.0 & 200000 & & 2662 \\
\hline NEC SX-7/160M5(1.81ns) & 160 & 1378 & 200000 & 15200 & 1412.8 \\
\hline $\begin{array}{l}\text { Dell PowerEdge } 1850 \text { (Xeon } 64 \text { 3.2GHz w/Topspin } \\
\text { InfiniBand) }\end{array}$ & 256 & 1349 & 220440 & 110220 & 1638 \\
\hline Intel ASCI Option Red (200 MHz Pentium Pro) & 9152 & 1338. & 235000 & 63000 & 1830 \\
\hline Legend DeepComp 1800 (2GHz Pentium 4 w/Myrinet) & 512 & 1297 & 172000 & & 2048 \\
\hline $\begin{array}{l}\text { Self Made Pentium4 Xeon (80-3.06 GHz, 72-2.8 GHz, } \\
112-2.4 \mathrm{GHz}, 256-2.2 \mathrm{GHz} \text { w/GigE) }\end{array}$ & 520 & 1283 & 260000 & & 2557 \\
\hline Intel EM64T (2 way $3.2 \mathrm{GHz}$ Intel EM64T w/Myrinet D) & 256 & 1269 & 241920 & & 1638 \\
\hline $\begin{array}{l}\text { IBM Power } 760 \text { ( } 3.4 \mathrm{GHz} \text { POWER7+, AIX, Intelligent } \\
\text { Energy Optimization enabled, up to } 3.787 \mathrm{GHz})\end{array}$ & 48 & 1268 & 217600 & 9344 & 1454 \\
\hline $\begin{array}{l}\text { IBM Power } 760 \text { (3.4 GHz POWER7+, SLES11SP2, } \\
\text { Intelligent Energy Optimization enabled, up to } 3.787 \mathrm{GHz} \text { ) }\end{array}$ & 48 & 1259.0 & 232223 & 9600 & 1454 \\
\hline HP Integrity rx2600 Itanium2 (1.3 GHz w/Myrinet) & 304 & 1253 & 256000 & & 1580 \\
\hline IBM eServer (Opteron $2.2 \mathrm{GHz}$ w/Infiniband) & 400 & 1246 & 200000 & & 1760 \\
\hline $\begin{array}{l}\text { IBM eServer HS20 cluster ( } 2 \text { way } 3.2 \mathrm{GHz} \text { Intel Xeon EM64T } \\
\text { w/Myrinet) }\end{array}$ & 252 & 1196 & 160922 & & 1612.8 \\
\hline NEC SX-5/128M8(3.2ns) & 128 & 1192.0 & 129536 & 10240 & 1280 \\
\hline $\begin{array}{l}\text { SGI Altix } 4700 \text { (Intel Itanium2 (Montvale) @ } 1.66 \mathrm{GHz} \\
\text { processor cores w/NUMAlink interconnect) }\end{array}$ & 200 & 1183 & 164640 & 32000 & 1328 \\
\hline IBM xSeries Cluster Dual Xeon (3.06 GHz w/Myrinet) & 490 & 1172 & 180000 & & 1499 \\
\hline $\begin{array}{l}\text { Visual Technology SuperNova / AMD Opteron } 1.8 \mathrm{GHz} \\
\text { GigtEth }\end{array}$ & 512 & 1169.0 & 220000 & 59000 & 1843.2 \\
\hline
\end{tabular}




\begin{tabular}{|c|c|c|c|c|c|}
\hline $\begin{array}{c}\text { Computer } \\
\text { (Full Precision) }\end{array}$ & $\begin{array}{l}\text { Number } \\
\text { of Procs } \\
\text { or Cores }\end{array}$ & $\begin{array}{c}\boldsymbol{R}_{\max } \\
\text { GFlop/s }\end{array}$ & $\begin{array}{l}N_{\max } \\
\text { Order }\end{array}$ & $\begin{array}{c}N_{1 / 2} \\
\text { Order }\end{array}$ & $\begin{array}{c}\boldsymbol{R}_{\text {Peak }} \\
\text { GFlop/s }\end{array}$ \\
\hline Cray X-1 (800 MHz) & 100 & 1167.1 & 217600 & 23040 & 1280.0 \\
\hline SGI Altix Itanium $21300 \mathrm{MHz}$ & 256 & 1142 & 334080 & 46000 & 1331.2 \\
\hline NEC SX-6/128M16(1.77ns) & 128 & 1141.0 & 327680 & 8960 & 1152 \\
\hline HP rx2600 Itanium2 (1.3 GHz w/Myrinet) & 304 & 1137 & 240000 & & 1580.8 \\
\hline $\begin{array}{l}\text { Bull NovaScale } 516016 \times 16 \text { Itanium2 1.3GHz w/Quadrics } \\
\text { Elan } 4\end{array}$ & 256 & 1131 & 335872 & 32000 & 1331 \\
\hline NEC SX-6/144M18 (2 ns) & 144 & 1130 & 225216 & 11232 & 1152 \\
\hline $\begin{array}{l}\text { IBM eServer Opteron e } 325 \text { Cluster ( } 2 \text { way, } 2.2 \mathrm{GHz} \text { Opteron } \\
\text { w/Myrinet) }\end{array}$ & 352 & 1128 & 185800 & & 1548.8 \\
\hline CRAY T3E-1200 (600 MHz) & 1488 & 1127 & 148800 & 28272 & 1786 \\
\hline $\begin{array}{l}\text { IBM eServer Opteron e } 325 \text { Cluster ( } 2 \text { way, } 2.2 \text { GHz AMD } \\
\text { Opteron w/Myrinet) }\end{array}$ & 352 & 1120 & 185000 & & 1548.8 \\
\hline IBM eServer pSeries 655 (32x8-way $1.7 \mathrm{GHz}$ POWER4+) & 256 & 1107 & 224000 & 14000 & 1740.8 \\
\hline $\begin{array}{l}\text { IBM eServer Blade Center Cluster HS20 (dual } 3.2 \text { GHz Xeon } \\
\text { EM64T w/GigE) }\end{array}$ & 252 & 1104 & 160000 & & 1613 \\
\hline Dell 1750 cluster Intel Xeon (dual $3.06 \mathrm{GHz}$ w/Gnet) & 304 & 1095 & 175000 & 40000 & 1860 \\
\hline Intel P4 Xeon (3.06 GHz w/Myrinet 2000) & 252 & 1084 & 247000 & 38000 & 1542 \\
\hline Intel ASCI Option Red (200 MHz Pentium Pro) & 7264 & 1068. & 215000 & 53400 & 1453 \\
\hline Linux Networx dual Intel Xeon (3.06GHz w/Myrinet) & 256 & 1060 & 159432 & 29858 & 1566.72 \\
\hline IBM AVIDD-B+AVIDD-I(2.4 GHz Xeon Force10) & 384 & 1058. & 220000 & & 1843 \\
\hline Linux Networx Dual AMD Opteron (1.8 GHz w/Infiniband) & 512 & 1053 & 114000 & 22202 & 1843 \\
\hline Cray X-1 (800 MHz) & 90 & 1050.1 & 195840 & 23808 & 1152.0 \\
\hline IBM Power 595 (5.0 GHz POWER6 SLES 11) & 64 & 1050 & 130000 & 9500 & 1280 \\
\hline IBM xSeries Cluster Xeon 2.4 GHz w/Gig-E & 448 & 1040 & 195000 & & 2150 \\
\hline IBM eServer pSeries 690 Turbo(1.3 GHz Power 4) & 384 & 1038. & 245000 & & 1997 \\
\hline Intel Pentium 4 dual-Xeon 2.8Ghz w/Quadrics Elan3 & 256 & 1036 & 170000 & 30600 & 1434 \\
\hline HITACHI SR8000-F1/112(375MHz) & 112 & 1035.0 & 120000 & 15160 & 1344 \\
\hline $\begin{array}{l}\text { IBM eServer Opteron e } 325 \text { Cluster ( } 2 \text { way, } 2.0 \mathrm{GHz} \text { AMD } \\
\text { Opteron w/GigE) }\end{array}$ & 432 & 1034 & 215000 & & 1728 \\
\hline Fujitsu SPARC Enterprise M9000 (2.4 GHz dual core) & 128 & 1032.0 & 331045 & 48108 & 1228.8 \\
\hline Sun SPARC Enterprise M9000 (2.4 GHz dual core) & 128 & 1032.0 & 331045 & 48108 & 1228.8 \\
\hline IBM Power 595 (5.0 GHz POWER6 RHEL 5.2) & 64 & 1032 & 120000 & 9100 & 1280 \\
\hline $\begin{array}{l}\text { IBM eServer } 1350 \text {-xSeries } 335 \text { ( } 2 \text { way } 3.06 \mathrm{GHz} \text { Xeon } \\
\text { w/Infiniband) }\end{array}$ & 252 & 1032 & 180000 & & 1542 \\
\hline Cray X-1 (800 MHz) & 88 & 1029.6 & 202752 & 23936 & 1126.4 \\
\hline IBM Power 595 (5.0 GHz POWER6) & 64 & 1028 & 183800 & 17000 & 1280 \\
\hline Self Made P4(256/2.2GHz+112/2.4GHz+32/2.53w/Genet) & 400 & 1011 & 257912 & & 1843 \\
\hline HP Compaq AlphaServer SC ES40/833 (833 MHz) & 812 & 1007 & 252700 & 39954 & 1352.8 \\
\hline Linux NetworX/Quadrics(2.4 GHz Xeon w/Myrinet) & 391 & 1007 & 208000 & 25000 & 1732 \\
\hline $\begin{array}{l}\text { Galactic Computing ( } 2.8 \mathrm{Ghz} \text { Pentium } 4 \text { Xeon, w/InfiniBand } \\
4 \mathrm{x})\end{array}$ & 264 & 1003 & 153000 & 30850 & 1478.4 \\
\hline $\begin{array}{l}\text { IMSc-Netweb-Summation Intel dual Xeon } 2.4 \mathrm{GHz} \\
\text { w/Dolphin 3D SCI }\end{array}$ & 288 & 1002 & 183000 & & 1382.4 \\
\hline $\begin{array}{l}\text { IBM eServer Opteron e } 325 \text { Cluster (dual } 2.0 \mathrm{GHz} \text { AMD } \\
\text { Opteron w/GigE) }\end{array}$ & 432 & 987.1 & 215000 & & 1728 \\
\hline $\begin{array}{l}\text { IBM PowerLinux 7R4 (4.0 GHz POWER7+, RHEL 6.4, } \\
\text { Intelligent Energy Optimization enabled, up to } 4.431 \mathrm{GHz})\end{array}$ & 32 & 987.1 & 160000 & 9000 & 1134 \\
\hline
\end{tabular}




\begin{tabular}{|c|c|c|c|c|c|}
\hline $\begin{array}{c}\text { Computer } \\
\text { (Full Precision) }\end{array}$ & $\begin{array}{l}\text { Number } \\
\text { of Procs } \\
\text { or Cores }\end{array}$ & $\begin{array}{l}\boldsymbol{R}_{\max } \\
\text { GFlop/s }\end{array}$ & $\begin{array}{l}N_{\max } \\
\text { Order }\end{array}$ & $\begin{array}{c}N_{1 / 2} \\
\text { Order }\end{array}$ & $\begin{array}{c}\boldsymbol{R}_{\text {Peak }} \\
\text { GFlop/s }\end{array}$ \\
\hline $\begin{array}{l}\text { IBM Power } 750 \text { Express ( } 4.0 \mathrm{GHz} \text { POWER } 7+\text {, AIX, } \\
\text { Intelligent Energy Optimization enabled, up to } 4.431 \mathrm{GHz})\end{array}$ & 32 & 984.5 & 163200 & 8930 & 1134 \\
\hline NEC SX-6/128M16 & 128 & 982.0 & 204800 & 12800 & 1024 \\
\hline $\begin{array}{l}\text { IBM Power } 750 \text { Express ( } 4.0 \mathrm{GHz} \text { POWER7+, SLES11SP2, } \\
\text { Intelligent Energy Optimization enabled, up to } 4.431 \mathrm{GHz} \text { ) }\end{array}$ & 32 & 980.2 & 160000 & 7400 & 1134 \\
\hline IBM eServer pSeries 690 Turbo (1.7 GHz POWER4+) & 256 & 976.0 & 280000 & & 1740.8 \\
\hline Intel dual Xeon $2.4 \mathrm{GHz}$ w/Dolphin 3D SCI & 288 & 970.3 & 182000 & & 1382 \\
\hline Linux NetworX/Quadrics(2.4 GHz Xeon w/Myrinet) & 352 & 962.8 & 200000 & 33000 & 1690 \\
\hline Self-made Intel Pentium $2.2 \mathrm{GHz}$ w/SCI3D & 400 & 960.4 & 220800 & 32800 & 1760 \\
\hline Self-made Intel Dual Xeon 2.4 GHz w/Dolphin 3D SCI & 288 & 957.1 & 184000 & 33050 & 1382.4 \\
\hline $\begin{array}{l}\text { IBM eServer } 1350-x \text { Series } 335 \text { ( } 2 \text { way } 3.06 \mathrm{GHz} \text { Xeon } \\
\text { w/Infiniband) }\end{array}$ & 256 & 947.7 & 110000 & & 1566.7 \\
\hline Cray X-1 (800 MHz) & 80 & 937.0 & 194560 & 21376 & 1024.0 \\
\hline $\begin{array}{l}\text { SunFire X4200 \& X4100 (Dual Core AMD Opteron(tm) } 2.39 \\
\text { GHz Processor } 280 \text { w/Infiniband SDR 4x (10Gb)) }\end{array}$ & 256 & 934.5 & 157184 & & 1196.6 \\
\hline SP Power3 $375 \mathrm{MHz}$ Nighthawk 2 & 1056 & 929.8 & 220000 & 62000 & 1584 \\
\hline NEC SX-6/120M15(2ns) & 120 & 927.6 & 204000 & 19440 & 960 \\
\hline $\begin{array}{l}\text { IBM eServer Opteron e } 325 \text { Cluster ( } 2 \text { way, } 2.0 \text { GHz AMD } \\
\text { Opteron w/GigE) }\end{array}$ & 432 & 926.6 & 201600 & & 1728 \\
\hline HITACHI SR8000-F1/100(375MHz) & 100 & 917.2 & 115000 & 15000 & 1200 \\
\hline IBM XSeries Xeon 2.8GHz, NPACI-ROCKS, Myrinet & 256 & 916.5 & 150000 & 25000 & 1433.6 \\
\hline IBM p690 cluster, Power $41.3 \mathrm{GHz}$ & 360 & 910 & 210000 & & 1872 \\
\hline $\begin{array}{l}\text { IBM Power } 750 \text { Express ( } 3.5 \mathrm{GHz} \text { POWER7+, AIX, } \\
\text { Intelligent Energy Optimization enabled, up to } 3.955 \mathrm{GHz} \text { ) }\end{array}$ & 32 & 895.7 & 163200 & 8930 & 1012 \\
\hline $\begin{array}{l}\text { IBM eServer xSeries Linux cluster ( } 2.4 \mathrm{GHz} \text { Pentium IV } \\
\text { Xeon w/ Gigabit Ethernet) }\end{array}$ & 768 & 894.9 & 210000 & & 3686.4 \\
\hline CRAY T3E-1200E (600 MHz) & 1080 & 891.5 & 259200 & 26400 & 1296 \\
\hline Fujitsu VPP5000/100 (3.33nsec) & 100 & 886.0 & 195600 & 18000 & 960 \\
\hline HITACHI SR8000/128(250MHz) & 128 & 873.6 & 120000 & 16000 & 1024 \\
\hline $\begin{array}{l}\text { IBM Power } 750 \text { Express ( } 3.55 \mathrm{GHz} \text { POWER7, RHEL 6, } \\
\text { Intelligent Energy Optimization enabled, up to } 3.86 \mathrm{GHz} \text { ) }\end{array}$ & 32 & 870.0 & 150016 & 7680 & 989 \\
\hline IBM eServer xSeries Linux (2.4GHz P4 Xeon) & 768 & 868.6 & 200000 & & 3686 \\
\hline SGI Origin 3000 (R14000A $600 \mathrm{MHz})$ & 1024 & 852.9 & 129024 & 31744 & 1229 \\
\hline HP XC1 Itanium 2 (1 GHz w/Quadrics) & 256 & 851.0 & 232000 & 24650 & 1024 \\
\hline Grendels dual $2.4 \mathrm{GHz}$ Intel Xeons w/Myrinet & 252 & 840.5 & 175760 & 27768 & 1210 \\
\hline $\begin{array}{l}\text { IBM eServer 1350-xSeries } 335 \text { ( } 2 \text { way Xeon } 2.4 \mathrm{GHz} \\
\text { w/Myrinet) }\end{array}$ & 250 & 829.8 & 154000 & & 1200 \\
\hline IBM eServer pSeries 690 (1.1 GHz Power4) & 512 & 826.5 & 185000 & 60000 & 2253 \\
\hline $\begin{array}{l}\text { Apple G5 dual } 2.0 \mathrm{GHz} \text { IBM Power PC } 970 \text { s, Infiniband } 4 \mathrm{X} \\
\text { primary fabric, Cisco Gigabit Ethernet secondary fabric }\end{array}$ & 256 & 821 & 120000 & & 1024 \\
\hline CRAY T3E-900 (450 MHz) & 1320 & 815.1 & 134400 & 26880 & 1188 \\
\hline Compaq AlphaServer SC ES45/1Ghz & 512 & 809 & 215000 & 27000 & 1024 \\
\hline HITACHI SR8000-G1/64(450MHz) & 64 & 790.7 & 110000 & 8504 & 921.6 \\
\hline Compaq AlphaServer SC ES45/1GHz & 480 & 772 & 140000 & 22950 & 960 \\
\hline LANL Space Simulator(Intel P4 $2.53 \mathrm{GHz}+1 \mathrm{~Gb})$ & 288 & 757.1 & 180000 & 44000 & 1457 \\
\hline $\begin{array}{l}\text { HP Integrity Superdome (1.6GHz/24MB Dual Core Itanium } \\
\text { 2) }\end{array}$ & 128 & 745.5 & 240000 & 27040 & 819.2 \\
\hline
\end{tabular}




\begin{tabular}{|c|c|c|c|c|c|}
\hline $\begin{array}{c}\text { Computer } \\
\text { (Full Precision) }\end{array}$ & $\begin{array}{l}\text { Number } \\
\text { of Procs } \\
\text { or Cores }\end{array}$ & $\begin{array}{c}\boldsymbol{R}_{\max } \\
\text { GFlop/s }\end{array}$ & $\begin{array}{c}N_{\max } \\
\text { Order }\end{array}$ & $\begin{array}{c}N_{1 / 2} \\
\text { Order }\end{array}$ & $\begin{array}{c}\boldsymbol{R}_{\text {Peak }} \\
\text { GFlop/s }\end{array}$ \\
\hline IBM eServer pSeries 690 Turbo(1.3 GHz Power 4) & 256 & 736.6 & 285000 & 25000 & 1331 \\
\hline Legend Group DeepComp 1800 - P4 Xeon 2.4 GHz - Myrinet & 256 & 735.8 & 114920 & 28000 & 1228.8 \\
\hline Self-Made MVS1000M EV67 (667 MHz Myrinet) & 768 & 734.6 & 270000 & 30000 & 1024 \\
\hline Fujitsu VPP5000/80 (3.33nsec) & 80 & 730.2 & 273600 & 15360 & 768.0 \\
\hline IBM eServer pSeries 655/651(1.1GHz Power 4) & 256 & 726.3 & 300000 & 20000 & 1126 \\
\hline IBM SP 176 nodes ( $375 \mathrm{MHz})$ & 704 & 723.4 & 187000 & 37500 & 1056 \\
\hline $\begin{array}{l}\text { Self-made MVS-5000BM Cluster IBM PowerPC } 970 \\
\text { (1.6 GHz w/Myrinet) }\end{array}$ & 84 & 722.1 & 200000 & 33000 & 1075.2 \\
\hline Presto III Athlon MP 1900+(1.6Ghz Myrinet) & 480 & 716.1 & 100000 & & 1536 \\
\hline Cray X-1 (800 MHz) & 60 & 706.3 & 168960 & 18432 & 768.0 \\
\hline Compaq AlphaServer SC ES45/1GHz & 480 & 706.0 & 205000 & 31400 & 960 \\
\hline IBM eServer pSeries 690 Turbo(1.3 GHz Power 4) & 224 & 704.8 & 135000 & & 1165 \\
\hline IBM eServer pSeries 690 Turbo(1.3 GHz Power 4) & 256 & 701.5 & 224000 & & 1331 \\
\hline HITACHI SR8000-E1/80(300MHz) & 80 & 691.3 & 120000 & 9408 & 768 \\
\hline IBM Cluster 1350 (208 proc 2.4GHz P4 Xeon) & 208 & 682.6 & 170000 & & 998.4 \\
\hline NCSA Titan Cluster(Itanium $800 \mathrm{MHz}$ w/Myrinet) & 320 & 677.9 & 183000 & 32000 & 1024 \\
\hline CRAY X1 (800 MHz, 60 procs) & 60 & 675.5 & 168960 & 17610 & 768.0 \\
\hline SGI Altix $3000(1.5 \mathrm{GHz})$ & 128 & 668.3 & 224000 & & 768 \\
\hline LANL Space Simulator P4(2.53GHz)+1000Mb/sw & 288 & 665.1 & 180000 & 65000 & 1457 \\
\hline Compaq AlphaServer SC ES45/1GHz & 480 & 660.8 & 210000 & 47000 & 960 \\
\hline $\begin{array}{l}\text { Self-made MVS-5000BM Cluster IBM JS20, IBM PowerPC } \\
9701.6 \mathrm{GHz} \text { w/Myrinet }\end{array}$ & 152 & 655.5 & 185000 & 29000 & 972.8 \\
\hline NEC Magi Cluster (PIII 933 MHz w/Myrinet) & 1012 & 654.8 & 217600 & 29000 & 944 \\
\hline Intel Pentium 4(dual 2.0 GHz w/Myrinet 2000) & 240 & 654.7 & 159000 & & 960 \\
\hline SGI Altix 3000 (Itanium 2, $1.5 \mathrm{GHz}$ ) & 128 & 651.7 & 160000 & 160000 & 768 \\
\hline $\begin{array}{l}\text { IBM eServer Opteron Cluster (2-way Opteron } 2.0 \mathrm{GHz} \\
\text { w/GigE) }\end{array}$ & 240 & 651.4 & 166000 & 43200 & 960 \\
\hline IBM eServer pSeries 690 (1.5 GHz POWER4+) & 192 & 651.4 & 220000 & & 1152.0 \\
\hline Pentium 4 (256-2.2GHz,72-2.4GHz,32-2.8GHz) & 360 & 644. & 234000 & & 1651 \\
\hline HP Superdome (1.5GHz Itanium 2, w/HyperPlex & 128 & 642.9 & 235040 & 68000 & 768.0 \\
\hline IBM x335 Cluster dual Xeon 2.8 GHz + GIG-E & 258 & 638.8 & 160000 & 65000 & 1433.6 \\
\hline $\begin{array}{l}\text { IBM Flex System p270 ( } 3.4 \mathrm{GHz} \text { POWER7+, RHEL 6.4, } \\
\text { Intelligent Energy Optimization enabled, up to } 3.787 \mathrm{GHz} \text { ) }\end{array}$ & 24 & 635 & 160000 & 7000 & 727 \\
\hline IBM eServer xSeries Cluster(2.8 GHz Pentium 4) & 184 & 629.7 & 103000 & 20700 & 1030 \\
\hline SGI Origin 3000 (R14000 $500 \mathrm{MHz}$ ) & 768 & 623.2 & 163000 & 25000 & 768 \\
\hline RWC (933MHz 512-dual Pent III w/Myrinet2000) & 1012 & 618.3 & 146000 & 23000 & 955.4 \\
\hline Dell 2650 Windows(Pentium 42.4 GHz w/Gnet) & 256 & 618.0 & 166000 & 50000 & 1228 \\
\hline IBM BladeCenter Xeon Dual Processor 2.4 GHz GigE & 280 & 613.9 & 200000 & & 1344 \\
\hline IBM SP 140 nodes (222 MHz POWER3) & 1120 & 613.02 & 170000 & 50000 & 994.6 \\
\hline HITACHI SR8000-F1/64(375MHz) & 64 & 605.3 & 92000 & 10048 & 768 \\
\hline Intel Pentium 4(dual 2.0 GHz w/Myrinet 2000) & 256 & 605.2 & 154000 & & 1024 \\
\hline SGI Altix 3000 (Itanium 2, $1.3 \mathrm{GHz}$ ) & 128 & 594.9 & 320000 & 320000 & 665.6 \\
\hline Linux Cluster UIUC-NCSA (1 GHz Pentium III) & 1008 & 594.5 & 235000 & & 1008 \\
\hline
\end{tabular}




\begin{tabular}{|c|c|c|c|c|c|}
\hline $\begin{array}{c}\text { Computer } \\
\text { (Full Precision) }\end{array}$ & $\begin{array}{l}\text { Number } \\
\text { of Procs } \\
\text { or Cores }\end{array}$ & $\begin{array}{c}\boldsymbol{R}_{\max } \\
\text { GFlop/s }\end{array}$ & $\begin{array}{c}N_{\max } \\
\text { Order }\end{array}$ & $\begin{array}{c}N_{1 / 2} \\
\text { Order }\end{array}$ & $\begin{array}{c}\boldsymbol{R}_{\text {Peak }} \\
\text { GFlop/s }\end{array}$ \\
\hline $\begin{array}{l}\text { MEGWARE Computer GmbH (dual Intel Xeon } 3.06 \mathrm{GHz} \text {, } \\
\text { FSB533, 8Gb/s Infiniband) }\end{array}$ & 128 & 593.7 & 154856 & 16432 & 783.36 \\
\hline IBM eServer pSeries 690 Turbo(1.3 GHz Power 4) & 256 & 590.2 & 158000 & 158000 & 1331 \\
\hline ASCI Red Intel Pentium II Xeon core 333Mhz & 2336 & 581.1 & 180864 & 31500 & 778 \\
\hline HITACHI SR8000-F1/60(375MHz) & 60 & 577.5 & 89000 & 10000 & 720 \\
\hline Apple Xserve G5 (2GHz PowerPC 970 w/Myrinet) & 88 & 575.4 & 114000 & & 704 \\
\hline Cray X-1 (800 MHz) & 49 & 572.5 & 150528 & 15232 & 627.2 \\
\hline Compaq Alpha 21264A(667MHz,dual w/Myrinet) & 742 & 564.2 & 230000 & 37440 & 989.8 \\
\hline Fujitsu VPP5000/64 (3.33nsec) & 64 & 563.0 & 235776 & 12288 & 614.4 \\
\hline $\begin{array}{l}\text { IBM BladeCenter PS704 Express ( } 2.46 \mathrm{GHz} \text { POWER7, } \\
\text { RHEL 6,Intelligent Energy Optimization not enabled) }\end{array}$ & 32 & 560.5 & 160000 & 7552 & 630.78 \\
\hline $\begin{array}{l}\text { IBM BladeCenter PS704 Express ( } 2.46 \mathrm{GHz} \text { POWER7, } \\
\text { RHEL 6, Intelligent Energy Optimization not enabled) }\end{array}$ & 32 & 559.6 & 160000 & 7552 & 630.78 \\
\hline IBM eServer pSeries 690 Turbo(1.3 GHz Power 4) & 192 & 558.2 & 245000 & 22000 & 998.4 \\
\hline IBM SP 120 nodes (222 MHz POWER3) & 960 & 558.13 & 200000 & 53000 & 852.5 \\
\hline HITACHI SR8000-E1/64(300MHz) & 64 & 556.5 & 110000 & 8000 & 614 \\
\hline IBM eServer pSeries 655 (16x8-way $1.7 \mathrm{GHz}$ POWER4+) & 128 & 556.5 & 156000 & 10000 & 870.4 \\
\hline IBM pSeries 690 Turbo(7x32 1.3GHz w/Gigenet) & 224 & 555.3 & 226800 & & 1165 \\
\hline SGI Origin $3000,700 \mathrm{MHz}$ & 512 & 553.0 & 230000 & 230000 & 717 \\
\hline CRAY X1 (800 MHz, 49 procs) & 49 & 550.5 & 150528 & 16128 & 627.2 \\
\hline Fujitsu M8000 (SPARC64 VII 2.52GHz, quad core) & 64 & 548.2 & 156200 & 12000 & 645.12 \\
\hline Sun M8000 (SPARC64 VII 2.52GHz, quad core) & 64 & 548.2 & 156200 & 12000 & 645.12 \\
\hline RWC SCore Cluster III Pentium III (933MHz) & 960 & 547.9 & 140000 & 24000 & 955.4 \\
\hline IBM SP 475 nodes (332 MHz 604e) & 1900 & 547.0 & 244000 & 58000 & 1262 \\
\hline IBM SP 32 nodes (375 MHz POWER3 High) & 512 & 546.3 & 148000 & 33000 & 768.0 \\
\hline SGI Origin $2000(250 \mathrm{MHz})$ & 1536 & 543.2 & 203904 & 64512 & 768 \\
\hline IBM eServer xSeries Linux (2.4GHz P4 Xeon) & 512 & 540.2 & 224200 & & 2458 \\
\hline IBM SP 128 nodes (375 MHz POWER3 Thin) & 512 & 538.4 & 163000 & & 768 \\
\hline Bull Novascale 5160 (8x16 Itanium2 $1.3 \mathrm{GHz}$ w/Quadrics) & 128 & 535.9 & 236544 & 36864 & 665.6 \\
\hline $\begin{array}{l}\text { PARAM Padma C-DAC IBM p630(Quad } \\
\text { P4-1.0GHz)w/PARAMNet-II }\end{array}$ & 248 & 532.2 & 224000 & 43895 & 992 \\
\hline $\begin{array}{l}\text { IBM x } 3850 \text { X5 [Dual Chassis configuration with QPI (Quick } \\
\text { Path Interconnect) (Intel Xeon X7560@ } 2.27 \text { GHz, } 64 \text { cores } \\
\text { ( } 8 \text { sockets * } 8 \text { cores) }\end{array}$ & 64 & 526 & 168000 & & 581 \\
\hline ASCI Red Intel Pentium II Xeon core $-333 \mathrm{Mhz}$ & 2336 & 522.5 & 121856 & 25300 & 778 \\
\hline $\begin{array}{l}\text { IBM Power } 740 \text { Express ( } 4.2 \mathrm{GHz} \text { POWER7+, AIX, } \\
\text { Intelligent Energy Optimization enabled, up to } 4.540 \mathrm{GHz})\end{array}$ & 16 & 517.1 & 117504 & 3500 & 581 \\
\hline $\begin{array}{l}\text { IBM Power } 730 \text { Express ( } 4.2 \mathrm{GHz} \text { POWER } 7+\text {, AIX, } \\
\text { Intelligent Energy Optimization enabled, up to } 4.540 \mathrm{GHz} \text { ) }\end{array}$ & 16 & 514.3 & 117504 & 3500 & 581 \\
\hline CPlant/Ross(Alpha EV6 466 MHz w/Myrinet) & 1000 & 512.43 & 142300 & & 932 \\
\hline Compaq AlphaServer SC ES45/1Ghz & 324 & 512 & 170000 & 20000 & 648 \\
\hline $\begin{array}{l}\text { IBM PowerLinux 7R2 (4.2 GHz POWER7+, SLES11SP2, } \\
\text { Intelligent Energy Optimization enabled, up to } 4.540 \mathrm{GHz})\end{array}$ & 16 & 508.2 & 112128 & 4000 & 581 \\
\hline $\begin{array}{l}\text { IBM Power } 730 \text { Express ( } 4.2 \mathrm{GHz} \text { POWER7+, SLES11SP2, } \\
\text { Intelligent Energy Optimization enabled, up to } 4.540 \mathrm{GHz} \text { ) }\end{array}$ & 16 & 508.5 & 112128 & 4000 & 581 \\
\hline $\begin{array}{l}\text { IBM Power } 740 \text { Express ( } 4.2 \mathrm{GHz} \text { POWER7+, SLES11SP2, } \\
\text { Intelligent Energy Optimization enabled, up to } 4.540 \mathrm{GHz} \text { ) }\end{array}$ & 16 & 508.5 & 115383 & 4200 & 581 \\
\hline
\end{tabular}




\begin{tabular}{|c|c|c|c|c|c|}
\hline $\begin{array}{c}\text { Computer } \\
\text { (Full Precision) }\end{array}$ & $\begin{array}{l}\text { Number } \\
\text { of Procs } \\
\text { or Cores }\end{array}$ & $\begin{array}{c}\boldsymbol{R}_{\max } \\
\text { GFlop/s }\end{array}$ & $\begin{array}{l}N_{\max } \\
\text { Order }\end{array}$ & $\begin{array}{c}N_{1 / 2} \\
\text { Order }\end{array}$ & $\begin{array}{c}\boldsymbol{R}_{\text {Peak }} \\
\text { GFlop/s }\end{array}$ \\
\hline Compaq ES40/EV67 AlphaServer SC & 512 & 507.6 & 200000 & 30000 & 683 \\
\hline IBM Power 575 (4.7 GHz POWER6 RHEL 5.2) & 32 & 500.0 & 105400 & 7000 & 601.6 \\
\hline IBM eServer pSeries 655 (8-way 1.5 GHz POWER4+) & 128 & 498.5 & 224000 & 15000 & 768 \\
\hline $\begin{array}{l}\text { BM Flex System p260 ( } 4.1 \mathrm{GHz} \text { POWER } 7+\text {, AIX, Intelligent } \\
\text { Energy Optimization enabled, up to } 4.340 \mathrm{GHz}) 16\end{array}$ & 496 & 117504 & 3500 & & 555 \\
\hline NEC SX-6/64M8 & 64 & 495.2 & 122880 & 6656 & 512 \\
\hline Fujitsu VPP5000/56 (3.33nsec) & 56 & 492.4 & 228480 & 12768 & 538 \\
\hline $\begin{array}{l}\text { IBM Flex System p260 (4.1 GHz POWER7+, SLES11SP2, } \\
\text { Intelligent Energy Optimization enabled, up to } 4.340 \mathrm{GHz} \text { ) }\end{array}$ & 16 & 485.4 & 112128 & 4300 & 555 \\
\hline IBM eServer pSeries 690 (1.1 GHz Power4) & 256 & 485.2 & 158000 & & 1126 \\
\hline Fujitsu VPP800/63 (4.0nsec) & 63 & 482.5 & 234360 & 12852 & 504 \\
\hline AMD Athlon MP2000+ cluster(1.667GHz,w/Fenet) & 240 & 480.7 & 116100 & 24570 & 800 \\
\hline IBM SP 28 nodes (375 MHz POWER3 High) & 448 & 480.4 & 138000 & 31000 & 672.0 \\
\hline SKIF K-500 Pentium Xeon 2.8 GHz SCI 3D & 128 & 475.3 & 123000 & 18304 & 716.8 \\
\hline PARRAM Padma (IBM p630 w/PARAMNet-II) & 240 & 475.0 & 230400 & 72850 & 960 \\
\hline HP Superdome (750 MHz, HyperPlex) & 256 & 470.93 & 340092 & 90072 & 768 \\
\hline IBM ASCI Option Blue Pacific (332 MHz) & 1344 & 468.2 & 205000 & 65000 & 892 \\
\hline Sun Fire Supercluster $(1050 \mathrm{MHz} 3 \times 100)$ & 300 & 468.1 & 230400 & 38400 & 630 \\
\hline IBM Power 575 (4.7 GHz POWER6) & 32 & 467 & 110000 & 9000 & 602 \\
\hline SGI Origin 3000 (R14000A $600 \mathrm{MHz})$ & 512 & 466.0 & 111104 & 19840 & 614.4 \\
\hline $\begin{array}{l}\text { IBM eServer Blade Center JS20 (2-way PowerPC970 1.6Ghz } \\
\text { w/GigE) }\end{array}$ & 164 & 462 & 140000 & & 1049.6 \\
\hline HITACHI SR8000/64(250MHz) & 64 & 449.7 & 92000 & 9160 & 512 \\
\hline HP Superdome (750 MHz, 1000bT) & 256 & 449.44 & 340092 & 110052 & 768 \\
\hline IBM Power 570 (4.2 GHz POWER6) & 32 & 449.2 & 110000 & 18000 & 537.6 \\
\hline CRAY T3E (300 MHz) & 1024 & 448.6 & 119808 & 19008 & 614 \\
\hline CRAY T3E-1200E (600 MHz) & 540 & 447.8 & 181440 & 17280 & 648 \\
\hline IBM SP 96 nodes (222 MHz POWER3) & 768 & 446.20 & 183000 & 45000 & 682.0 \\
\hline IBM xSeries(2.8 GHz Intel P4 w/Myrinet 2000) & 126 & 443.7 & 125000 & & 705.6 \\
\hline CRAY T3E-900 (450 MHz) & 690 & 443.1 & 144000 & 18720 & 621 \\
\hline IBM Power 740 Express (3.55 GHz POWER7) & 16 & 439.3 & 130400 & 400 & 494.6 \\
\hline $\begin{array}{l}\text { IBM Power } 740 \text { Express ( } 3.55 \mathrm{GHz} \text { POWER7, RHEL 6, } \\
\text { Intelligent Energy Optimization enabled, up to } 3.86 \mathrm{GHz} \text { ) }\end{array}$ & 16 & 435.8 & 112129 & 5200 & 494 \\
\hline IBM Power 730 Express (3.55 GHz POWER7) & 16 & 435.4 & 112128 & 5376 & 494 \\
\hline IBM Power 570 (4.2 GHz POWER6 RHEL 5.2) & 32 & 433.7 & 110000 & 7000 & 537.6 \\
\hline NEC SX-6/56M7 & 56 & 433.6 & 107520 & 5824 & 448 \\
\hline IBM Power 730 Express (3.55 GHz POWER7) & 16 & 432.9 & 92000 & 4000 & 494.6 \\
\hline Cray X-1 (800 MHz) & 36 & 422.1 & 129024 & 13056 & 460.8 \\
\hline Dell Cluster (2.4 GHz XEON w/Myrinet) & 128 & 421.9 & 117200 & & 614.4 \\
\hline Sun HPC 4500 Cluster/64 (400MHz/8MB L2) & 896 & 420.44 & 144000 & 43200 & 716.8 \\
\hline Intel Itanium 2 (1.3 GHz Quad proc w/Myrinet 2000) & 96 & 418.4 & 136000 & 18000 & 499.2 \\
\hline IBM eServer p5 595 (1.9GHz POWER5) & 64 & 418.0 & 152000 & 8000 & 486.4 \\
\hline IBM eServer p5 595 (1.9GHz POWER5) & 64 & 416.8 & 157000 & 12000 & 486.4 \\
\hline $\begin{array}{l}\text { Intel dual Pentium Xeon }(768-3.06 \mathrm{GHz} \& 252-3.2 \mathrm{GHz} \\
\text { w/Myrinet) }\end{array}$ & 1020 & 415.2 & 321600 & & 631.1 \\
\hline
\end{tabular}




\begin{tabular}{|c|c|c|c|c|c|}
\hline $\begin{array}{c}\text { Computer } \\
\text { (Full Precision) }\end{array}$ & $\begin{array}{l}\text { Number } \\
\text { of Procs } \\
\text { or Cores }\end{array}$ & $\begin{array}{c}\boldsymbol{R}_{\max } \\
\text { GFlop/s }\end{array}$ & $\begin{array}{l}N_{\max } \\
\text { Order }\end{array}$ & $\begin{array}{c}N_{1 / 2} \\
\text { Order }\end{array}$ & $\begin{array}{c}\boldsymbol{R}_{\text {Peak }} \\
\text { GFlop/s }\end{array}$ \\
\hline CRAY T3E-900 (450 MHz) & 640 & 413.7 & 138240 & 18432 & 576 \\
\hline SGI Origin $3000(500 \mathrm{MHz})$ & 512 & 405.60 & 230000 & 130560 & 512 \\
\hline CRAY X1 (800 MHz, 36 procs) & 36 & 404.3 & 129024 & 12416 & 460.8 \\
\hline AMD Athlon $1900+1.6 \mathrm{GHz}$ Myrinet-2000 & 240 & 403.6 & 142000 & 26000 & 768 \\
\hline Self-made Xenia / IBM Intellistation(Xeon $2.4 \mathrm{GHz}$ ) Myrinet & 128 & 401.4 & 85000 & 14600 & 614.4 \\
\hline HITACHI SR8000-G1/32(450MHz) & 32 & 395.6 & 85000 & 5320 & 460.8 \\
\hline IBM eServer xSeries Linux (2.4GHz P4 Xeon) & 256 & 381.1 & 158600 & & 1229 \\
\hline SGI Origin $2000(250 \mathrm{MHz})$ & 1024 & 379.6 & 164736 & 40500 & 512 \\
\hline SGI Altix 3000, $900 \mathrm{Mhz}$ & 128 & 378.9 & 110400 & 30000 & 461 \\
\hline IBM eServer pSeries 690 Turbo(1.3 GHz Power 4) & 128 & 378.2 & 200000 & 16000 & 665.6 \\
\hline $\begin{array}{l}\text { IBM BladeCenter PS702 Express ( } 3.00 \mathrm{GHz} \text { POWER7, } \\
\text { RHEL 6, Intelligent Energy Optimization enabled, up to } 3.30 \\
\text { GHz) }\end{array}$ & 16 & 375.7 & 112128 & 6408 & 423 \\
\hline NEC SX-6/48M6 & 48 & 374.5 & 107520 & 4992 & 384 \\
\hline CP-PACS* (150 MHz PA-RISC based CPU) & 2048 & 368.2 & 103680 & 30720 & 614 \\
\hline Intel Pentium III (572@1GHz, 452@1.266GHz) & 1024 & 366.0 & 242000 & & 1144 \\
\hline NEC SX-5/48M3 (4 nsec) & 48 & 364.6 & 76800 & & 384 \\
\hline IBM eServer pSeries 655/651(1.1GHz Power 4) & 128 & 364.5 & 210000 & 13000 & 563 \\
\hline IBM xSeries 2.8 Ghz x335 Pentium IV Linux cluster & 128 & 361.6 & 112000 & & 716.8 \\
\hline Fujitsu VPP5000/38 (3.33nsec) & 38 & 351.1 & 196080 & 9120 & 364.8 \\
\hline IBM SP (200 MHz Power 3 nodes) & 768 & 350.4 & 113000 & 30000 & 614 \\
\hline $\begin{array}{l}\text { Intel Pentium4 1.7GHz(1) / 2.0GHz(98) / 2.4GHz(44)/ } \\
2.53 \mathrm{GHz}(35) / 2.8 \mathrm{GHz}(4) \text { Giganet }\end{array}$ & 182 & 349.3 & 144800 & & 806.1 \\
\hline Compaq AlphaServer SC ES40/833 & 256 & 344.1 & 142000 & 17000 & 427 \\
\hline PowerEdge 2650(P-4,2GHz+120 P-4,2.2GHz w/Gnet) & 180 & 343.4 & 100000 & & 768 \\
\hline CRAY T3E (300 MHz) & 784 & 342.8 & 104832 & 17280 & 470 \\
\hline HP Superdome (1.5GHz Itanium 2, 6.0MB L3 Cache) & 64 & 341.7 & 154080 & 15040 & 384.0 \\
\hline SGI Altix 3000 (Itanium 2, $1.5 \mathrm{GHz}$ ) & 64 & 338.0 & 160000 & 160000 & 384 \\
\hline HP Superdome (1.5GHz Itanium2, 6MB L3 Cache) & 64 & 335.45 & 150080 & 15200 & 384.0 \\
\hline IBM Power 730 Express(3.7 GHz POWER7) & 12 & 335.2 & 108000 & 440 & 376.3 \\
\hline Self Made P4(95/2GHz+341/2.4GHz+32/2.53 w/Gnet) & 168 & 334.9 & 138990 & 40000 & 739 \\
\hline $\begin{array}{l}\text { IBM Power } 730 \text { Express ( } 3.72 \mathrm{GHz} \text { POWER7, RHEL 6, } \\
\text { Intelligent Energy Optimization enabled, up to } 3.92 \mathrm{GHz} \text { ) }\end{array}$ & 12 & 333.1 & 112128 & 5504 & 376.32 \\
\hline Compaq AlphaServer SC ES40/EV67 $833 \mathrm{MHz}$ & 256 & 332.2 & 192000 & 20000 & 426.5 \\
\hline Athlon MP 1.2Ghz, w/Myrinet 2000 & 252 & 331.7 & 90720 & & 614.4 \\
\hline Cray X-1 (800 MHz) & 28 & 329.8 & 114688 & 12160 & 358.4 \\
\hline PowerEdge HPC Cluster (2.4 GHz Xeon w/Gnet) & 198 & 327.9 & 100000 & & 950 \\
\hline Compaq AlphaServerSC ES40(833 MHz Quadrics) & 300 & 326.4 & 110000 & 38000 & 499.8 \\
\hline Xenia/IBM Intellistation(Xeon 2.4 GHz w/Myrinet) & 128 & 323.4 & 86000 & 14600 & 614.4 \\
\hline Helix(Cardiff 108@2.2 GHz, 36@2.4 GHzw/Dophin) & 144 & 322.5 & 105000 & 17000 & 648 \\
\hline CRAY T3E-900 (450 MHz) & 512 & 321.1 & 122880 & 15360 & 461 \\
\hline Fujitsu VPP700/160E (6.5nsec) & 160 & 319.4 & 168000 & 24000 & 384 \\
\hline CRAY X1 (800 MHz, 28 procs) & 28 & 318.1 & 114688 & 11302 & 358.4 \\
\hline IBM xSeries Xeon Dual Processor $2.4 \mathrm{GHz}$ & 168 & 317.8 & 137000 & & 806.4 \\
\hline
\end{tabular}




\begin{tabular}{|c|c|c|c|c|c|}
\hline $\begin{array}{c}\text { Computer } \\
\text { (Full Precision) }\end{array}$ & $\begin{array}{l}\text { Number } \\
\text { of Procs } \\
\text { or Cores }\end{array}$ & $\begin{array}{c}\boldsymbol{R}_{\max } \\
\text { GFlop/s }\end{array}$ & $\begin{array}{c}N_{\max } \\
\text { Order }\end{array}$ & $\begin{array}{c}N_{1 / 2} \\
\text { Order }\end{array}$ & $\begin{array}{c}\boldsymbol{R}_{\text {Peak }} \\
\text { GFlop/s }\end{array}$ \\
\hline SGI Origin $3000400 \mathrm{MHz}, 512 \mathrm{CPU}$ & 512 & 315.5 & 130560 & 108800 & 409.6 \\
\hline HITACHI SR8000-F1/32(375MHz) & 32 & 313.3 & 65000 & 6000 & 384 \\
\hline IBM SP 256 nodes (332 MHz 604e) & 1024 & 311.9 & 180000 & 40000 & 680 \\
\hline NEC SX-6/40M5 & 40 & 311.7 & 102400 & 4480 & 320 \\
\hline CPlant/Siberia(Alpha EV6 500 MHz w/Myrinet) & 552 & 309.2 & 105700 & & 552 \\
\hline Dell PowerEdge HPC(P4 2.4 GHz Xeon w/Myrinet) & 128 & 308.3 & 115000 & & 614.4 \\
\hline IBM SP 64 nodes (222 MHz POWER3) & 512 & 307.63 & 148000 & 35000 & 454.7 \\
\hline IBM pSeries 690 Turbo(4x32 1.3GHz w/Gigenet) & 128 & 306.4 & 112000 & & 665.8 \\
\hline SGI Origin 3000 (500 MHz, $384 \mathrm{CPU})$ & 384 & 306.30 & 384000 & 96768 & 967.7 \\
\hline SGI Origin $2800(400 \mathrm{MHz})$ & 512 & 300.23 & 130560 & 21216 & 409.6 \\
\hline SGI Origin 2000 (400 MHz, $512 \mathrm{CPU})$ & 512 & 300.20 & 130560 & 130560 & 409.6 \\
\hline SGI Altix 3000 (Itanium 2, $1.3 \mathrm{GHz}$ ) & 64 & 297.2 & 160000 & 160000 & 332.8 \\
\hline Fujitsu VPP5000/32 (3.33nsec) & 32 & 296.1 & 170880 & 7680 & 307 \\
\hline IBM SP 256 nodes (200 MHz POWER3) & 512 & 287.84 & 140000 & 30000 & 410 \\
\hline COMPAS-ECCO (Pentium III, 1GHZ w/Myrinet) & 480 & 285.9 & 150000 & 17000 & 480 \\
\hline Compaq AlphaServer SC ES45/1GHz (ev68) & 176 & 285.3 & 124000 & 14000 & 352 \\
\hline Intel Paragon XP/S MP (50 MHz OS=SUNMOS) & 6768 & 281.1 & 128600 & 25700 & 338 \\
\hline Dell Precision 530(Pentium 4-1.7 GHz, GigE) & 208 & 280.4 & 96000 & & 707 \\
\hline NEC SX-7/32 (1.81ns) & 32 & 280.3 & 72000 & 2064 & 282.5 \\
\hline SGI Origin $3000,700 \mathrm{MHz}$ & 256 & 279.9 & 163000 & 163000 & 358 \\
\hline HP rx26000 Itanium2 $1.3 \mathrm{GHz}$ Cluster w/InfiniBand & 64 & 278.7 & 98304 & 9216 & 332.8 \\
\hline IBM SP 16 nodes (375 MHz POWER3 High) & 256 & 278.3 & 107000 & 21200 & 384.0 \\
\hline Dell PowerEdge HPC Cluster(2.4 GHz Xeon w/genet) & 128 & 277.8 & 115000 & 30000 & 614 \\
\hline IBM Power 570 (5.0 GHz POWER6) & 16 & 277.7 & 104000 & 5600 & 320.6 \\
\hline IBM eServer xSeries Linux (2.4GHz P4 Xeon) & 128 & 274.0 & 112000 & & 614.4 \\
\hline Sun HPC4500 Cluster/60 (336MHz/4MB L2) & 720 & 272.1 & 192000 & & 483.8 \\
\hline Compaq Alphaserver SC512(500Mhz w/Quadrics) & 512 & 271.4 & 140000 & & 512 \\
\hline Fujitsu VPP700/128E (6.5nsec) & 128 & 268.9 & 166400 & 23040 & 307 \\
\hline Fujitsu SPARC Enterprise M9000 (2.4 GHz) & 32 & 268.6 & 162085 & 6500 & 307.2 \\
\hline Sun SPARC Enterprise M9000 (2.4 GHz) & 32 & 268.6 & 162085 & 6500 & 307.2 \\
\hline Compaq ES40/EV67 AlphaServer SC & 256 & 263.6 & 106000 & 20000 & 342 \\
\hline hp server rp8400 (750 MHz, HyperPlex) & 128 & 261.09 & 234144 & 50004 & 384 \\
\hline IBM eServer pSeries 690 (1.1 GHz Power4) & 128 & 259.5 & 112000 & 21000 & 563.2 \\
\hline IBM SP 64 nodes (375 MHz POWER3 Thin) & 256 & 257.82 & 148000 & 24000 & 384 \\
\hline Intel Paragon XP/S MP (50 MHz OS=SUNMOS) & 6144 & 256.2 & 122500 & 24300 & 307 \\
\hline HITACHI SR8000/36(250MHz) & 36 & 255.9 & 69000 & 5968 & 288 \\
\hline Fujitsu SPARC Enterprise M9000 (2.28 GHz) & 32 & 255.3 & 158045 & 6500 & 291.8 \\
\hline Sun SPARC Enterprise M9000 (2.28 GHz) & 32 & 255.3 & 158045 & 6500 & 291.8 \\
\hline NEC SX-6 & 32 & 253.6 & 76800 & 3328 & 256 \\
\hline NEC SX-6/32M4 & 32 & 251.2 & 92160 & 3584 & 256 \\
\hline hp server rp8400 (750 MHz, 1000bT) & 128 & 251.11 & 234144 & 70092 & 384 \\
\hline HP Superdome (750 MHz, HyperPlex) & 128 & 248.90 & 220104 & 36072 & 384 \\
\hline IBM eServer pSeries 655 (8-way $1.5 \mathrm{GHz}$ POWER4+) & 64 & 248.7 & 160000 & 11000 & 384 \\
\hline
\end{tabular}




\begin{tabular}{|c|c|c|c|c|c|}
\hline $\begin{array}{c}\text { Computer } \\
\text { (Full Precision) }\end{array}$ & $\begin{array}{l}\text { Number } \\
\text { of Procs } \\
\text { or Cores }\end{array}$ & $\begin{array}{c}\boldsymbol{R}_{\max } \\
\text { GFlop/s }\end{array}$ & $\begin{array}{c}N_{\max } \\
\text { Order }\end{array}$ & $\begin{array}{c}N_{1 / 2} \\
\text { Order }\end{array}$ & $\begin{array}{c}\boldsymbol{R}_{\text {Peak }} \\
\text { GFlop/s }\end{array}$ \\
\hline NEC SX-5/32M2 (4 nsec) & 32 & 247.0 & 55296 & & 256 \\
\hline HP Superdome (750 MHz, 1000bT) & 128 & 245.11 & 220968 & 43092 & 384 \\
\hline SGI Origin $3000($ R14000A $600 \mathrm{MHz})$ & 256 & 245.1 & 120000 & 120000 & 307.2 \\
\hline SGI Origin $2000300 \mathrm{MHz}, 512 \mathrm{CPU}$ & 512 & 241.40 & 147456 & 33984 & 307.2 \\
\hline IBM Power 570 (4.7GHz, POWER6) & 16 & 239.4 & 92000 & 4400 & 301 \\
\hline IBM Power 570 (4.7GHz POWER6) & 16 & 239.4 & 92000 & 4400 & 300.8 \\
\hline LosLobos Supercluster(PIII 733MHz w/Myrinet) & 500 & 237.0 & 150000 & 20000 & 366.5 \\
\hline IBM Power 570 (4.7 GHz POWER6) & 16 & 235.1 & 90000 & 7230 & 300.8 \\
\hline CRAY T3E (300 MHz) & 540 & 234.9 & 86400 & 14400 & 324 \\
\hline HELIX (AMD 1.76GHz w/gnet) & 132 & 234.8 & 82080 & 25000 & 466 \\
\hline HITACHI SR2201/1024(150MHz) & 1024 & 232.3 & 155520 & 34560 & 307 \\
\hline Numerical Wind Tunnel* (9.5 ns) & 167 & 229.7 & 66132 & 18018 & 281 \\
\hline HITACHI SR8000/32(250MHz) & 32 & 229.5 & 65000 & 5632 & 256 \\
\hline IBM Power 570 (4.7 GHz POWER6 RHEL 5.1) & 16 & 229.4 & 110000 & 8400 & 300.8 \\
\hline Intel Paragon XP/S MP (50 MHz OS=SUNMOS) & 5376 & 223.6 & 114500 & 22900 & 269 \\
\hline CRAY T3E (300 MHz) & 512 & 222.3 & 84480 & 12480 & 307 \\
\hline CLiC (Pentium III 800 MHZ) & 529 & 221.6 & 176640 & 28272 & 423.2 \\
\hline Korean Inst S\&T(Pentium 4 1.7GHz w/Myrinet) & 128 & 221.6 & 115000 & 18000 & 435.2 \\
\hline SGI Altix 3000, $1 \mathrm{Ghz}$ & 64 & 219.4 & 167039 & 167039 & 256 \\
\hline IBM eServer p5 595 (1.9GHz POWER5) & 32 & 217.1 & 130000 & 9000 & 243.2 \\
\hline Fujitsu VPP700/116(7nsec) & 116 & 213.0 & 111360 & 18560 & 255 \\
\hline Titech Grid Cluster, Pentium III-S 1.4Ghz & 256 & 212.7 & 115000 & & 358.4 \\
\hline CRAY T3E-1200E (600 MHz) & 256 & 211.8 & 125952 & 11520 & 307 \\
\hline Compaq SC232 (667 MHz) & 232 & 211.0 & 120000 & & 309.5 \\
\hline IBM SP 128 nodes (332 MHz 604e) & 512 & 210.2 & 100000 & 20872 & 340 \\
\hline SGI Origin $3000(500 \mathrm{MHz})$ & 256 & 210.20 & 163200 & 163000 & 256 \\
\hline HITACHI SR8000-F1/20(375MHz) & 20 & 206.15 & 68000 & 4440 & 240 \\
\hline Intel EPG (dual 3.06GHz Xeon w/Myrinet) & 64 & 202.7 & 100000 & & 391.7 \\
\hline Fujitsu VPP500/153(10nsec) & 153 & 200.6 & 62730 & 17000 & 245 \\
\hline HITACHI SR8000-G1/16(450MHz) & 16 & 199.1 & 62000 & 3440 & 230.4 \\
\hline Self Made(91-P4 2GHz + 35-P4 2.4GHz w/Genet) & 126 & 198.7 & 85000 & 30000 & 532.0 \\
\hline IBM ASCI Option Blue Pacific (332 MHz) & 672 & 198.6 & 95000 & 37000 & 446 \\
\hline SGI Origin 3000 Cluster2x128(R14000A $600 \mathrm{MHz})$ & 256 & 198.5 & 160000 & 160000 & 307.2 \\
\hline SGI Altix 3000, $900 \mathrm{MHz}$ & 64 & 197.4 & 119039 & 119039 & 230 \\
\hline Self-Made Intel Pentium 4 Xeon(1.7GHz w/GigE) & 208 & 197.2 & 90000 & & 707 \\
\hline HPTi ACL-276/667 (Alpha 667 MHz w/Myrinet) & 270 & 196.34 & 80000 & & 360 \\
\hline SGI Origin $2000(250 \mathrm{MHz})$ & 512 & 195.6 & 110592 & 23040 & 256 \\
\hline Numerical Wind Tunnel* (9.5 ns) & 140 & 195.0 & 60480 & 15730 & 236 \\
\hline HP Integrity rx8640 (1.6GHz/24MB Dual-Core Itanium 2) & 32 & 192.4 & 116000 & 7520 & 204.8 \\
\hline Intel Paragon XP/S MP (50 MHz OS=SUNMOS) & 4608 & 191.5 & 106000 & 21000 & 230 \\
\hline IBM eServer pSeries 690 Turbo(1.3 GHz Power 4) & 64 & 191.4 & 148000 & 11000 & 332.8 \\
\hline NEC SX-6/24M3 & 24 & 188.7 & 69120 & 2688 & 192 \\
\hline Cray X-1 (800 MHz) & 16 & 188.5 & 81920 & 8064 & 204.8 \\
\hline
\end{tabular}




\begin{tabular}{|c|c|c|c|c|c|}
\hline $\begin{array}{c}\text { Computer } \\
\text { (Full Precision) }\end{array}$ & $\begin{array}{l}\text { Number } \\
\text { of Procs } \\
\text { or Cores }\end{array}$ & $\begin{array}{c}\boldsymbol{R}_{\max } \\
\text { GFlop/s }\end{array}$ & $\begin{array}{c}N_{\max } \\
\text { Order }\end{array}$ & $\begin{array}{c}N_{1 / 2} \\
\text { Order }\end{array}$ & $\begin{array}{c}\boldsymbol{R}_{\text {Peak }} \\
\text { GFlop/s }\end{array}$ \\
\hline IBM eServer p5 590 (1.65GHz POWER5) & 32 & 187.8 & 113000 & 5800 & 211.2 \\
\hline IBM eServer pSeries 655/651(1.1GHz Power 4) & 64 & 184.7 & 150000 & 9000 & 282 \\
\hline Netfinity Xseries(X330) PIII 1GHz & 320 & 184.4 & 120000 & 1500 & 320 \\
\hline IBM x330 Cluster PIII $1 \mathrm{GHz}$ w/100Mb enet & 420 & 182.4 & 192000 & 192000 & 420 \\
\hline CRAY X1 (800 MHz, 16 procs) & 16 & 182.3 & 81920 & 8242 & 204.8 \\
\hline NEC TX7/i9510 Itanium2 1.6GHz & 32 & 181.92 & 200848 & 7824 & 204.8 \\
\hline Numerical Wind Tunnel* (9.5 ns) & 128 & 179.2 & 56832 & 14800 & 216 \\
\hline Compaq AlphaServerSC ES40/EV68 833MHz & 160 & 178.0 & 71000 & 20000 & 266.5 \\
\hline Sun Fire $15 \mathrm{~K}(1050 \mathrm{MHz} / 8 \mathrm{MB} \mathrm{E} \$)$ & 106 & 177.2 & 206116 & 18000 & 222.6 \\
\hline NEC TX7/i9510 Itanium2 $1.5 \mathrm{GHz}$ & 32 & 172.30 & 161936 & 7440 & 192 \\
\hline SGI Altix 3000 (Itanium 2, $1.5 \mathrm{GHz}$ ) & 32 & 171.9 & 16000 & 16000 & 192.0 \\
\hline SGI Itanium 2, $800 \mathrm{MHz}$ & 64 & 171.8 & 115199 & 115199 & 204.8 \\
\hline HP 9000 Superdome (1000MHz PA-8800) & 64 & 171.8 & 120800 & 10000 & 256 \\
\hline IBM QS22 blade (2 PowerXCell 8i processors) & 18 & 170.7 & 48895 & & 217.6 \\
\hline Fujitsu VPP500/128(10nsec) & 128 & 170.2 & 56832 & 14804 & 205 \\
\hline Intel Pentium III (1 GHz w/100 Mb enet) & 512 & 169.4 & 16000 & & 512 \\
\hline Sun Fire 15K (1050MHz/8MB E\$) & 104 & 168.5 & 96116 & 17000 & 218.4 \\
\hline IBM S80s (450 MHz, SP switch) & 360 & 167.87 & 113000 & 31000 & 324 \\
\hline Compaq AlphaServer SC ES40/EV67 (667MHz) & 184 & 167.5 & 99900 & 22500 & 245.5 \\
\hline Self Made(6-P4 1.7GHz + 99-P4 2GHz w/Genet) & 105 & 167.2 & 77900 & 27000 & 416.4 \\
\hline Origin $3000400 \mathrm{MHz}$ Cluster(2x128) & 256 & 167.1 & 204800 & 163000 & 204.8 \\
\hline IBM eServer pSeries 690 (1.1 GHz Power4) & 64 & 163.8 & 148000 & & 332.8 \\
\hline SGI Origin $3000400 \mathrm{MHz}, 256 \mathrm{CPU}$ & 256 & 163.5 & 163200 & 81920 & 204.8 \\
\hline IBM eServer pSeries 690(2x32w/Genet $1.3 \mathrm{GHz})$ & 64 & 161.9 & 80000 & & 281.6 \\
\hline CRAY T3E-900 (450 MHz) & 256 & 161.6 & 84480 & 10080 & 230 \\
\hline Intel P 4 cluster $(92-2.0 \mathrm{GHz}+6-1.7 \mathrm{GHz}$ w/Genet $)$ & 98 & 160.4 & 75500 & 24000 & 388 \\
\hline HITACHI SR8000-F1/16(375MHz) & 16 & 159.5 & 46000 & 3800 & 192 \\
\hline Pentium 4 (2 GHz w/Giganet) & 91 & 157.8 & 73500 & 26000 & 364 \\
\hline IBM S80s (450 MHz, SP switch) & 336 & 157.75 & 109000 & 29000 & 302 \\
\hline Sun Fire 15K (1050 MHz/8MB E\$) & 96 & 157.6 & 96116 & 16000 & 201.6 \\
\hline IBM SP 32 nodes (222 MHz POWER3) & 256 & 157.46 & 107000 & 25000 & 227.3 \\
\hline Compaq AS SC256 (500 MHz EV6 Quadrics sw) & 256 & 154.4 & 120000 & 26000 & 256 \\
\hline SGI Origin $2000400 \mathrm{MHz}, 256 \mathrm{CPU}$ & 256 & 152.20 & 163200 & 163200 & 204.8 \\
\hline SGI Altix 3000 (Itanium 2, $1.3 \mathrm{GHz}$ ) & 32 & 151.8 & 160000 & 160000 & 166.4 \\
\hline IBM SP/472 (120 MHz) & 460 & 151.8 & 61000 & 22600 & 221 \\
\hline Intel Paragon XP/S MP (50 MHz OS=SUNMOS) & 3648 & 151.7 & 95000 & 18100 & 182 \\
\hline SGI Origin $3000(400 \mathrm{MHz})(2 \times 128 \mathrm{cpu})$ & 256 & 151.20 & 112640 & 112640 & 204.8 \\
\hline HITACHI SR8000-G1/12(450MHz) & 12 & 150.11 & 54000 & 3000 & 172.8 \\
\hline IBM SP 128 nodes (200 MHz POWER3) & 256 & 149.36 & 100000 & 18500 & 205 \\
\hline Sun Blade 1000 750MHz Cluster w/Myrinet2000 & 196 & 149.2 & 70560 & 70560 & 294 \\
\hline Compaq ES40/EV68 AlphaServer SC (833 MHz) & 128 & 149.1 & 70000 & & 213.2 \\
\hline Fujitsu VPP5000/16 (3.33nsec) & 16 & 149.1 & 120768 & 4416 & 154 \\
\hline Compaq AlphaServerSC ES40(833 MHz Quadrics) & 160 & 148. & 71000 & 2000 & 266.5 \\
\hline
\end{tabular}




\begin{tabular}{|c|c|c|c|c|c|}
\hline $\begin{array}{c}\text { Computer } \\
\text { (Full Precision) }\end{array}$ & $\begin{array}{l}\text { Number } \\
\text { of Procs } \\
\text { or Cores }\end{array}$ & $\begin{array}{c}\boldsymbol{R}_{\max } \\
\text { GFlop/s }\end{array}$ & $\begin{array}{c}N_{\max } \\
\text { Order }\end{array}$ & $\begin{array}{c}N_{1 / 2} \\
\text { Order }\end{array}$ & $\begin{array}{c}\boldsymbol{R}_{\text {Peak }} \\
\text { GFlop/s }\end{array}$ \\
\hline SGI Origin $2000(250 \mathrm{MHz})$ & 384 & 147.1 & 96768 & 17280 & 192 \\
\hline IBM S80s (450 MHz, SP switch) & 312 & 146.26 & 104800 & 28000 & 281 \\
\hline Sun Fire $15 \mathrm{~K}(1050 \mathrm{MHz} / 8 \mathrm{MB} \mathrm{E} \$)$ & 88 & 144.6 & 96116 & 15000 & 184.8 \\
\hline HITACHI SR8000/20(250MHz) & 20 & 144.5 & 48000 & 4000 & 160 \\
\hline Intel Paragon XPS-140 (50 MHz OS=SUNMOS) & 3680 & 143.4 & 55700 & 20500 & 184 \\
\hline IBM eServer pSeries 690 Turbo(1.7GHz POWER4+) & 32 & 143.3 & 151000 & 5000 & 217.6 \\
\hline HP AlphaServer GS1280 7/1300 (1.3 GHz) & 64 & 142.8 & 122500 & & 166.4 \\
\hline NEC SX-6/16M2 (1.77ns) & 16 & 142.8 & 51200 & 2048 & 144 \\
\hline Cray X-1 (800 MHz) & 12 & 142.4 & 73728 & 7040 & 153.6 \\
\hline SGI Origin $2000(195 \mathrm{MHz})$ & 480 & 141.2 & 108864 & 21312 & 187 \\
\hline HITACHI SR8000-E1/16(300MHz) & 16 & 140.8 & 62000 & 3200 & 154 \\
\hline SGI 1100 Cluster (Dual Pentium III, 1 GHz) & 324 & 140.5 & 133000 & & 324 \\
\hline IBM SP 8 nodes (375 MHz POWER3 High) & 128 & 138.8 & 76000 & 16000 & 192.0 \\
\hline IBM Power 550 (5.0 GHz POWER6+) & 8 & 137.6 & 64200 & 1900 & 160.0 \\
\hline CRAY X1 (800 MHz, 12 procs) & 12 & 137.6 & 73728 & 6294 & 153.6 \\
\hline IBM S80s (450 MHz, SP switch) & 288 & 137.17 & 100800 & 26000 & 259 \\
\hline IBM Power 550 (5.0 GHz POWER6+ SLES 11) & 8 & 137.1 & 85000 & 3500 & 160.0 \\
\hline IBM eServer pSeries 690 Turbo(1.1 GHz) & 64 & 137.1 & 80000 & 13500 & 281.6 \\
\hline Compaq ES40/EV6 AlphaServer SC & 256 & 135.7 & 120000 & & 256 \\
\hline Fujitsu VPP500/100(10nsec) & 100 & 135.3 & 51000 & 12816 & 160 \\
\hline HP Superdome (750 MHz) & 64 & 133.82 & 138888 & & 192 \\
\hline IBM SP 32 nodes (375 MHz POWER3 Thin) & 128 & 132.75 & 107000 & 15400 & 192 \\
\hline hp server rp8400 (750 MHz, HyperPlex) & 64 & 132.71 & 137808 & 21384 & 192 \\
\hline hp server rp8400 (750 MHz, 1000bT) & 64 & 132.69 & 165456 & 29268 & 192 \\
\hline Sun Fire 15K (1050MHz/8MB E\$) & 80 & 132.6 & 96116 & 14000 & 168.0 \\
\hline Intel Itanium $21.3 \mathrm{GHz}$ & 32 & 132.5 & 73400 & & 166.4 \\
\hline Dell PowerEdge HPC(Dual Pentium III, 1 GHz) & 400 & 131.0 & 130000 & 65000 & 400 \\
\hline Fujitsu VPP500/96 (10nsec) & 96 & 129.5 & 49728 & 12430 & 154 \\
\hline Fujitsu VPP700/64 (7nsec) & 64 & 129.5 & 115200 & 12800 & 141 \\
\hline Paragon XP/S MP(1024 Nodes, OS=SUNMOS S1.6) & 3072 & 127.1 & 86000 & 17800 & 154 \\
\hline NEC SX-8/8 (2 GHz) & 8 & 126.2 & 30720 & & 128 \\
\hline NEC SX-5/16 (4 nsec) & 16 & 125.8 & 55296 & & 128 \\
\hline NEC SX-6/16M2 (2 nsec) & 16 & 125.70 & 51200 & 2240 & 128 \\
\hline SGI Origin $3000600 \mathrm{MHz}, 128 \mathrm{CPU}$ & 128 & 125.5 & 81920 & 81920 & 154 \\
\hline IBM eServer pSeries 655 (8-way $1.5 \mathrm{GHz}$ POWER4+) & 32 & 125.2 & 112000 & 6000 & 192 \\
\hline IBM S80s (450 MHz, SP switch) & 264 & 124.66 & 96800 & 25000 & 238 \\
\hline Sun HPC 10000 Cluster/4 (336 MHz, 4MB L2) & 256 & 123.9 & 80640 & 26880 & 172 \\
\hline HITACHI SR8000-G1/10(450MHz) & 10 & 123.4 & 49440 & 2648 & 144.0 \\
\hline Origin $3000400 \mathrm{MHz}$ Cluster(64+128) & 192 & 122.3 & 96000 & 111000 & 153.6 \\
\hline NEC SX-4/64M2 (8.0 ns) & 64 & 122.2 & 30080 & 4352 & 128 \\
\hline Compaq AlphaServer SC40 EV/67 667 MHz & 112 & 121.3 & 107520 & & 149 \\
\hline Dell PowerEdge Cluster W2K(Dual PIII,1GHz/Gnet) & 252 & 120.7 & 155000 & 50000 & 252 \\
\hline IBM Power 570 (4.7GHz, POWER6) & 8 & 120.6 & 58000 & 3400 & 150 \\
\hline
\end{tabular}




\begin{tabular}{|c|c|c|c|c|c|}
\hline $\begin{array}{c}\text { Computer } \\
\text { (Full Precision) }\end{array}$ & $\begin{array}{l}\text { Number } \\
\text { of Procs } \\
\text { or Cores }\end{array}$ & $\begin{array}{c}\boldsymbol{R}_{\max } \\
\text { GFlop/s }\end{array}$ & $\begin{array}{l}N_{\max } \\
\text { Order }\end{array}$ & $\begin{array}{c}N_{1 / 2} \\
\text { Order }\end{array}$ & $\begin{array}{c}\boldsymbol{R}_{\text {Peak }} \\
\text { GFlop/s }\end{array}$ \\
\hline IBM Power 570 (4.7GHz POWER6) & 8 & 120.6 & 58000 & 3400 & 150.4 \\
\hline Sun Fire $15 \mathrm{~K}(1050 \mathrm{MHz} / 8 \mathrm{MB} \mathrm{E} \$)$ & 72 & 119.8 & 96116 & 12500 & 151.2 \\
\hline IBM Power 570 (4.7 GHz POWER6) & 8 & 118.4 & 79680 & 4000 & 150.4 \\
\hline Linux cluster PIII $(1.0 \mathrm{GHz}, \mathrm{w} / 100 \mathrm{Mb} / \mathrm{s}$ enet $)$ & 256 & 118.1 & 157000 & 157000 & 256 \\
\hline Fujitsu PRIMEPOWER2000(675MHz) & 128 & 118.0 & 116480 & 43000 & 259.2 \\
\hline IBM Power 570 (4.7 GHz POWER6 RHEL 5.1) & 8 & 116.4 & 83000 & 4400 & 150.4 \\
\hline CRAY T3E-900 (450 MHz) & 192 & 116.0 & 51840 & 8448 & 171 \\
\hline HITACHI SR8000/16(250MHz) & 16 & 115.9 & 42928 & 3584 & 128 \\
\hline Fujitsu PRIMERGY CL460J (Pentium4 1.7GHz) & 64 & 115.7 & 40000 & 9000 & 217.6 \\
\hline Cray T3E-1350 (675 MHz) & 128 & 113.9 & 89088 & 7488 & 172.8 \\
\hline IBM S80s (450 MHz, SP switch) & 240 & 113.31 & 92000 & 24000 & 216 \\
\hline $\begin{array}{l}\text { IBM BladeCenter JS43 Express ( } 4.2 \text { GHz POWER6+ SLES } \\
\text { 11) }\end{array}$ & 8 & 113.1 & 65000 & 3300 & 134.4 \\
\hline CRAY T3E (300 MHz) & 256 & 112.8 & 59904 & 8832 & 154 \\
\hline SGI Altix 3000, $1 \mathrm{Ghz}$ & 32 & 111.9 & 100000 & 100000 & 128 \\
\hline IBM SP (160 MHz, P2SC) & 256 & 111.64 & 52000 & 13100 & 163 \\
\hline IBM System p5 575 (1.9GHz POWER5+) & 16 & 111.4 & 92400 & 1340 & 121.6 \\
\hline SGI 1100 Cluster (Pentium III 1GHz) & 266 & 110.4 & 119000 & & 266 \\
\hline Fujitsu VPP700/56 (7nsec) & 56 & 110.3 & 109200 & 10752 & 123 \\
\hline Sun UltraSPARC II 450MHz 40 E420R 4proc/node & 160 & 110.0 & 136080 & 136080 & 144 \\
\hline Fujitsu VPP500/80 (10nsec) & 80 & 109.8 & 46400 & 11030 & 128 \\
\hline SGI Origin $2000300 \mathrm{MHz}$ Cluster(2x128) & 256 & 109.5 & 81920 & 81920 & 153.6 \\
\hline Dell PowerEdge HPC (Dual Pentium III, 1 GHz) & 320 & 109.0 & 120000 & 60000 & 320 \\
\hline IBM SP 64 nodes (332 MHz 604e) & 256 & 108.1 & 81460 & 14180 & 170 \\
\hline SGI Origin $3000(500 \mathrm{MHz})$ & 128 & 106.9 & 81920 & 81920 & 128 \\
\hline Sun Fire $15 \mathrm{~K}(1050 \mathrm{MHz} / 8 \mathrm{MB} \mathrm{E} \$)$ & 64 & 106.9 & 96116 & 12000 & 134.4 \\
\hline Sun Fire E6900 (UltraSPARC IV 1.35 Ghz w/custom) & 48 & 106.6 & 141565 & 8900 & 129.6 \\
\hline CRAY T3E-1200E(600 MHz) & 128 & 106.0 & 89088 & 7488 & 154 \\
\hline IBM S80s (450 MHz, SP switch) & 216 & 104.92 & 87000 & 22000 & 194 \\
\hline IBM System p5 560Q (1.8 GHz POWER5+) & 16 & 104.7 & 87400 & 4080 & 115.2 \\
\hline IBM Power 550 (4.2GHz, POWER6) & 8 & 104.6 & 76000 & 1700 & 135 \\
\hline IBM Power 550 (4.2GHz POWER6) & 8 & 104.6 & 76000 & 1700 & 134.4 \\
\hline IBM Power 550 (4.2 GHz POWER6 RHEL 5.1) & 8 & 104.2 & 85000 & 6100 & 134.4 \\
\hline IBM System p5 560Q (1.8GHz POWER5) & 16 & 104.2 & 92300 & 1400 & 115.2 \\
\hline Sun Fire $15 \mathrm{~K}(900 \mathrm{MHz} / 8 \mathrm{MB} \mathrm{L} 2 \$$, perflib) & 72 & 103.7 & 96116 & 10700 & 129.6 \\
\hline IBM eServer p5 570 (1900 MHz POWER5) & 16 & 103.1 & 72000 & 4000 & 121.60 \\
\hline IBM SP (375 MHz POWER3 ) & 90 & 102.8 & 90000 & & 135.0 \\
\hline Fujitsu PRIMEPOWER2000(563MHz) & 128 & 102.0 & 116480 & 44000 & 216 \\
\hline NEC TX7/i9510 (Itanium2,1GHz) & 32 & 101.77 & 128016 & 21840 & 128 \\
\hline SGI Origin $2000(250 \mathrm{MHz})$ & 256 & 101.4 & 86400 & 13248 & 128 \\
\hline HITACHI SR8000-G1/8(450MHz) & 8 & 101.3 & 44000 & 2432 & 115.2 \\
\hline Compaq AlphaServer SC40 EV/67 667 MHz & 96 & 101.2 & 96000 & 10000 & 128 \\
\hline SGI Origin $3000500 \mathrm{MHz}, 128 \mathrm{CPU}$ & 128 & 101.0 & 115000 & & 128 \\
\hline Pentium 4 (2 GHz, Giganet + F-enet) & 56 & 100.7 & 55000 & 16000 & 224 \\
\hline
\end{tabular}




\begin{tabular}{|c|c|c|c|c|c|}
\hline $\begin{array}{c}\text { Computer } \\
\text { (Full Precision) }\end{array}$ & $\begin{array}{l}\text { Number } \\
\text { of Procs } \\
\text { or Cores }\end{array}$ & $\begin{array}{c}\boldsymbol{R}_{\max } \\
\text { GFlop/s }\end{array}$ & $\begin{array}{c}N_{\max } \\
\text { Order }\end{array}$ & $\begin{array}{c}N_{1 / 2} \\
\text { Order }\end{array}$ & $\begin{array}{c}\boldsymbol{R}_{\text {Peak }} \\
\text { GFlop/s }\end{array}$ \\
\hline Cray T3D $1024(150 \mathrm{MHz})$ & 1024 & 100.5 & 81920 & 10224 & 152 \\
\hline Sun Ultra HPC10000 Cluster/4(250 MHz,4MB L2) & 256 & 100.4 & 80640 & 22528 & 128 \\
\hline IBM SP (375 MHz POWER3 ) & 88 & 99.7 & 88000 & & 132.0 \\
\hline $\begin{array}{l}\text { SGI Origin } 2000250 / 300 \mathrm{MHz} \text { Cluster } \\
(2 \times 64 \times 250+2 \times 64 \times 300)\end{array}$ & 256 & 98.87 & 81920 & 81920 & 140.8 \\
\hline Sun Fire 6900 (UltraSPARC IV, $1.2 \mathrm{GHz}$ ) & 48 & 98.26 & 96116 & 8300 & 115.2 \\
\hline IBM Cell BE $(3.2 \mathrm{GHz}) * * * * *$ & 9 & 98.05 & 4096 & 1536 & $\begin{array}{c}14.6(64 \text { bit }) \\
204.8(32 \text { bit })\end{array}$ \\
\hline SGI Altix 3000, $900 \mathrm{MHz}$ & 32 & 97.67 & 82079 & 82079 & 115 \\
\hline HP Integrity rx7640 (1.6GHz/18MB Dual-Core Itanium 2) & 16 & 96.85 & 76520 & 4320 & 102.4 \\
\hline Kepler(192 PIII@650 MHz + 4 PIII@733 MHz) & 196 & 96.25 & 109760 & 12320 & 127.7 \\
\hline IBM SP (375 MHz POWER3 ) & 84 & 95.5 & 88000 & & 126.0 \\
\hline IBM eServer pSeries 690 Turbo(1.3 GHz Power 4) & 32 & 95.26 & 108000 & 7000 & 166.4 \\
\hline Cray X-1 (800 MHz) & 8 & 95.2 & 61440 & 5632 & 102.4 \\
\hline Fujitsu VPP700/46 ( 7nsec) & 46 & 94.3 & 100280 & 8280 & 101 \\
\hline SGI Origin 300 (500 MHz, w/Myrinet) & 128 & 94.15 & 81920 & 81920 & 128 \\
\hline HP 9000 rp8420-32 (1000MHz PA-8800) & 32 & 94.1 & 58960 & 5200 & 128 \\
\hline $\begin{array}{l}\text { ClearSpeed CSX600 Advance accelerator boards (dual } \\
\text { ClearSpeed boards each } 250 \mathrm{MHz} \text { ) (frontend HP ProLiant } \\
\text { DL380 G5, dual node Intel Xeon } 5100 \text { dual core, } 3 \mathrm{GHz} \text { ) }\end{array}$ & 6 & 93.3 & 45000 & & 240 \\
\hline SGI Origin $2000250 \mathrm{MHz}$ Cluster(2x128) & 256 & 92.99 & 81920 & 81920 & 128 \\
\hline NEC SX-4/48M2 (8.0 ns) & 48 & 92.63 & 30080 & 2688 & 96 \\
\hline Sun Ultra HPC10000 Cluster/4(250 MHz,4MB L2) & 244 & 92.6 & 80640 & 21504 & 122 \\
\hline Sun Fire $15 \mathrm{~K}(900 \mathrm{MHz} / 8 \mathrm{MB} \mathrm{L} 2 \$$, perflib) & 64 & 92.58 & 96116 & 10000 & 115.2 \\
\hline CRAY X1 (800 MHz, 8 procs) & 8 & 92.4 & 61440 & 4996 & 102.4 \\
\hline IBM eServer pSeries 655/651(1.1GHz Power 4) & 32 & 92.24 & 106000 & 6000 & 141 \\
\hline IBM eServer pSeries 690 Turbo (1300 MHz) & 32 & 91.32 & 72000 & 3800 & 166.4 \\
\hline CRAY T3E-1200E & 112 & 90.4 & 58368 & 6432 & 134 \\
\hline Fujitsu VPP500/64 (10nsec) & 64 & 89.3 & 41472 & 9820 & 102 \\
\hline Sun Fire E6900 (UltraSPARC IV 1.35 Ghz w/custom) & 40 & 89.03 & 119565 & 7300 & 108.0 \\
\hline HP Integrity rx8620 (1.5GHz Itanium2, 6MB L3 Cache) & 16 & 88.8 & 58600 & 4200 & 96.0 \\
\hline IBM SP2-T2 (66 MHz) & 512 & 88.4 & 73500 & 20150 & 136 \\
\hline Sun Ultra HPC10000 Cluster/4(250 MHz,4MB L2) & 224 & 87.94 & 80640 & 19200 & 112 \\
\hline IBM System p5 560Q (1.5GHz POWER5+) & 16 & 87.77 & 92400 & 1320 & 96.0 \\
\hline Compaq Alphaserver GS320 (731Mhz 4MB L2) & 128 & 87.51 & 110000 & 110000 & 170.8 \\
\hline IBM eServer p5 575 (1.5 GHz POWER5) & 16 & 87.34 & 71050 & 1320 & 96.0 \\
\hline Presto III Athlon Cluster(1.33GHz, Myrinet) & 78 & 87.25 & 75160 & 25000 & 207.5 \\
\hline Hewlett-Packard SuperDome $552 \mathrm{MHz}$ & 64 & 86.45 & 41000 & 3960 & 141.3 \\
\hline SGI Origin $3000400 \mathrm{MHz}, 128 \mathrm{CPU}$ & 128 & 85.44 & 65536 & 65536 & 102.4 \\
\hline IBM Cell BE $(2.1 \mathrm{GHz})^{* * * * *}$ & 9 & 84.52 & 3712 & 1792 & $\begin{array}{l}9.6(64 \mathrm{bit}) \\
134.4(32 \mathrm{bit})\end{array}$ \\
\hline Bull NovaScale 5160 Intel Itanium 2 (1.5 GHz) & 16 & 83.25 & 85760 & 4736 & 96 \\
\hline CRAY T3E (300 MHz) & 192 & 83.07 & 51840 & 7680 & 115 \\
\hline Sun Fire 6900 (UltraSPARC IV, $1.2 \mathrm{GHz}$ ) & 40 & 82.12 & 96116 & 6500 & 96.0 \\
\hline SGI Origin $3000400 \mathrm{MHz}, 256 \mathrm{CPU}$ & 256 & 81.90 & 81920 & 81920 & 102.5 \\
\hline SGI Origin $2000400 \mathrm{MHz}, 128 \mathrm{CPU}$ & 128 & 81.76 & 65536 & 65536 & 102.4 \\
\hline
\end{tabular}




\begin{tabular}{|c|c|c|c|c|c|}
\hline $\begin{array}{c}\text { Computer } \\
\text { (Full Precision) }\end{array}$ & $\begin{array}{l}\text { Number } \\
\text { of Procs } \\
\text { or Cores }\end{array}$ & $\begin{array}{c}\boldsymbol{R}_{\max } \\
\text { GFlop/s }\end{array}$ & $\begin{array}{c}N_{\max } \\
\text { Order }\end{array}$ & $\begin{array}{c}N_{1 / 2} \\
\text { Order }\end{array}$ & $\begin{array}{c}\boldsymbol{R}_{\text {Peak }} \\
\text { GFlop/s }\end{array}$ \\
\hline H-P e-vectra Pentium III $733 \mathrm{MHz}$ & 225 & 81.60 & 80370 & 23265 & 165.9 \\
\hline Origin $3000400 \mathrm{MHz}$ Cluster(2x64) & 128 & 81.56 & 153600 & 81920 & 102.4 \\
\hline Sun Fire $15 \mathrm{~K}$ (900MHz/8MB L2\$, perflib) & 56 & 81.27 & 96116 & 8400 & 100.8 \\
\hline IBM S80s (450 MHz, SP switch) & 168 & 80.87 & 77000 & 20000 & 151 \\
\hline IBM SP 16 nodes (222 MHz POWER3) & 128 & 80.83 & 76000 & 15000 & 113.7 \\
\hline Sun Fire $15 \mathrm{~K}(1050 \mathrm{MHz} / 8 \mathrm{MB} \mathrm{E} \$)$ & 48 & 80.75 & 96116 & 8500 & 100.8 \\
\hline HITACHI SR8000-F1/8(375MHz) & 8 & 80.25 & 30352 & 2504 & 96 \\
\hline Compaq AlphaserverSC $833 \mathrm{mhz}$ & 64 & 80.00 & 60000 & 10000 & 106.6 \\
\hline Sony PlayStation $3(3.2 \mathrm{GHz})^{* * * * *}$ & 7 & 79.9 & 2000 & 900 & $\begin{array}{c}11 \text { (64 bit) } \\
154 \text { (32 bit) }\end{array}$ \\
\hline CRAY T3E-900 (450 MHz) & 128 & 79.59 & 42240 & 6432 & 115 \\
\hline Sun HPC 10000 Cluster/2 (400MHz/4MB L2) & 128 & 79.36 & 57120 & 10752 & 102 \\
\hline Hitachi S-3000 cluster/412 (3x4) (2 ns) & 12 & 78.2 & 31120 & 4880 & 96 \\
\hline Presto III Athlon Cluster(1.33GHz, F-enet) & 78 & 77.4 & 75160 & 25000 & 207.5 \\
\hline IBM SP 64 nodes (200 MHz POWER3) & 128 & 76.77 & 89000 & 11500 & 102 \\
\hline Fujitsu-Siemens hpcLine(Pentium III,850 MHz) & 192 & 76.1 & 66720 & 12960 & 163.2 \\
\hline Sun Ultra HPC10000 Cluster/3(250 MHz,4MB L2) & 192 & 75.65 & 65520 & 19200 & 96 \\
\hline Sun Ultra HPC10000 Cluster/4(250 MHz,4MB L2) & 192 & 75.58 & 80640 & 16320 & 96 \\
\hline Fujitsu VPP5000/8 (3.33nsec) & 8 & 74.89 & 85440 & 2688 & 76.8 \\
\hline Intel Paragon XPS-140 (50 MHz) & 1872 & 72.9 & 55000 & 17500 & 94 \\
\hline SGI Origin $2000300 \mathrm{MHz}$ Cluster(128+32) & 160 & 72.57 & 61440 & 61440 & 96 \\
\hline NEC SX-6/8 (1.77ns) & 8 & 71.67 & 30720 & 800 & 72 \\
\hline Sun Fire E6900 (UltraSPARC IV 1.35 Ghz w/custom) & 32 & 71.60 & 119565 & 5900 & 86.4 \\
\hline HP AlphaServer GS1280 7/1300 (1.3 GHz) & 32 & 71.13 & 65536 & & 83.2 \\
\hline IBM S80s (450 MHz, SP switch) & 144 & 70.94 & 72000 & 18000 & 130 \\
\hline IBM SP 4 nodes (375 MHz POWER3 High) & 64 & 70.65 & 54000 & 11000 & 96.0 \\
\hline Sun Fire 15K (900MHz/8MB L2\$, perflib) & 48 & 69.88 & 96116 & 7500 & 86.4 \\
\hline IBM SP 16 nodes (375 MHz POWER3 Thin) & 64 & 67.78 & 76000 & 10400 & 96 \\
\hline Sun Fire $15 \mathrm{~K}(1050 \mathrm{MHz} / 8 \mathrm{MB} \mathrm{E} \$)$ & 40 & 67.52 & 96116 & 7500 & 84.0 \\
\hline Fujitsu VPP700/32 ( 7nsec) & 32 & 67.3 & 83200 & 5760 & 70 \\
\hline Sun HPC 10000 Cluster/2 (336 MHz, 4MB L2) & 128 & 66.93 & 57120 & 10080 & 86 \\
\hline NEC SX-4/32 (8.0 ns) $* * *$ & 32 & 66.53 & 15360 & 1792 & 64 \\
\hline IBM System p5 575 (2.2GHz POWER5+) & 8 & 66.44 & 57200 & 860 & 70.4 \\
\hline Sun Fire 6900 (UltraSPARC IV, 1.2 GHz) & 32 & 65.94 & 96116 & 5400 & 76.8 \\
\hline IBM eServer pSeries 670 (1.5GHz POWER4+) & 16 & 65.06 & 80000 & 1200 & 96.0 \\
\hline IBM Power 520 (4.7 GHz POWER6+) & 4 & 65.01 & 47600 & 840 & 75.2 \\
\hline RWC SCore Cluster II(Dual PIII 800MHz+Myrinet) & 132 & 64.7 & 58000 & 8000 & 105.6 \\
\hline IBM Power 520 (4.7 GHz POWER6+ SLES 11) & 4 & 64.42 & 60000 & 1900 & 75.2 \\
\hline Origin $3000600 \mathrm{MHz}, 64 \mathrm{CPU}$ & 64 & 64.15 & 81920 & 81920 & 76.8 \\
\hline IBM eServer pSeries 655 (8-way $1.5 \mathrm{GHz}$ POWER4+) & 16 & 64.07 & 80000 & 4000 & 96 \\
\hline Paragon XP/S MP(512 Nodes, OS=SUNMOS S1.6) & 1516 & 64.0 & 61000 & 12200 & 77 \\
\hline Compaq Alphaserver GS320 (731Mhz 4MB L2) & 64 & 63.81 & 60000 & 9000 & 85.4 \\
\hline Compaq ES40/EV67 AlphaServer SC & 64 & 63.8 & 60000 & 9000 & 85.4 \\
\hline NEC SX-8/4 (2 GHz) & 4 & 63.30 & 30720 & & 64 \\
\hline NEC SX-6/8 (2 nsec) & 8 & 63.21 & 30720 & 800 & 64 \\
\hline
\end{tabular}




\begin{tabular}{|c|c|c|c|c|c|}
\hline $\begin{array}{c}\text { Computer } \\
\text { (Full Precision) }\end{array}$ & $\begin{array}{l}\text { Number } \\
\text { of Procs } \\
\text { or Cores }\end{array}$ & $\begin{array}{l}\boldsymbol{R}_{\max } \\
\text { GFlop/s }\end{array}$ & $\begin{array}{l}N_{\max } \\
\text { Order }\end{array}$ & $\begin{array}{c}N_{1 / 2} \\
\text { Order }\end{array}$ & $\begin{array}{c}\boldsymbol{R}_{\text {Peak }} \\
\text { GFlop/s }\end{array}$ \\
\hline hp AlphaServer GS1280 7/1150(1.15 GHz) & 32 & 62.89 & 65536 & & 73.6 \\
\hline HP Kayak Intel Cluster (NT 550 MHz PIII) & 256 & 62.59 & 122500 & 20500 & 141 \\
\hline SGI Origin $2000(300 \mathrm{Mhz})$ & 128 & 62.25 & 60032 & 9000 & 77 \\
\hline Cray T932 (2.2 ns) $* * *$ & 32 & 61.8 & 16384 & 1280 & 58 \\
\hline NEC SX-4/32 (8.0 ns) & 32 & 61.77 & 20480 & 1688 & 64 \\
\hline IBM Power 570 (4.7GHz, POWER6) & 4 & 61.6 & 39200 & 660 & 75 \\
\hline IBM Power 570 (4.7GHz POWER6) & 4 & 61.56 & 39200 & 660 & 75.2 \\
\hline NEC SX-4/32M2 (8.0 ns) & 32 & 61.32 & 20480 & 2432 & 64 \\
\hline Alphleet (Alpha cluster/Myranet, $500 \mathrm{MHz}$ ) & 140 & 61.3 & 56000 & 22000 & 140 \\
\hline IBM Power 570 (4.7 GHz POWER6 RHEL 5.1) & 4 & 60.37 & 59000 & 3500 & 75.2 \\
\hline IBM Power 570 (4.7 GHz POWER6) & 4 & 60.08 & 55000 & 3000 & 75.2 \\
\hline Thinking Machines CM-5 & 1024 & 59.7 & 52224 & 24064 & 131 \\
\hline Hitachi S-3000 cluster/309 (3x3) (2 ns) & 9 & 59.0 & 26940 & 3180 & 72 \\
\hline IBM S80s (450 MHz, SP switch) & 120 & 58.97 & 65000 & 17000 & 108 \\
\hline Sun Fire $12 \mathrm{~K}(1050 \mathrm{MHz} / 8 \mathrm{MB} \mathrm{E} \$)$ & 36 & 58.92 & 66166 & 6500 & 75.6 \\
\hline HITACHI SR2201/256(150MHz) & 256 & 58.68 & 77760 & 13440 & 78 \\
\hline Sun Fire $15 \mathrm{~K}(900 \mathrm{MHz} / 8 \mathrm{MB}$ L2\$, perflib) & 40 & 58.41 & 96116 & 6500 & 72.0 \\
\hline HITACHI SR8000/8(250MHz) & 8 & 58.3 & 30352 & 2304 & 64 \\
\hline Fujitsu VPP700/26E (6.5nsec) & 26 & 58.0 & 74880 & 5200 & 62 \\
\hline IBM SP2 (160 MHz) & 128 & 57.24 & 39000 & 9180 & 82 \\
\hline $\begin{array}{l}\text { IBM BladeCenter JS23 Express (4.2 GHz POWER6+ SLES } \\
\text { 11) }\end{array}$ & 4 & 57.14 & 58000 & 2000 & 67.2 \\
\hline Cray T3E-1350 (675 MHz) & 64 & 57.0 & 62976 & 5040 & 86.4 \\
\hline Sun Ultra HPC10000 Cluster/3(250 MHz,4MB L2) & 144 & 56.97 & 65520 & 14400 & 72 \\
\hline IBM eServer p5 575 (1.9GHz POWER5) & 8 & 56.78 & 61000 & 2800 & 60.8 \\
\hline CRAY T3E (300 MHz) & 128 & 55.72 & 42240 & 5952 & 76.8 \\
\hline IBM eServer p5 575 (1.9GHz POWER5) & 8 & 56.67 & 57200 & 796 & 60.8 \\
\hline Sun HPC 450 Cluster/40(300MHz/2MB L2 cache) & 160 & 55.44 & 89600 & 22400 & 96.0 \\
\hline SGI2400(Origin 2000)Enet-Cluster(6x32 250MHz) & 192 & 54.68 & 99840 & 99840 & 96 \\
\hline IBM SP 32 nodes (332 MHz 604e) & 128 & 54.27 & 57600 & 9376 & 85 \\
\hline $\begin{array}{l}\text { IBM Blade Server: BladeCenter T-HS20 w/2.8 GHz Xeon } \\
\text { and GigE }\end{array}$ & 16 & 54.16 & 38000 & 7600 & 89.6 \\
\hline Hitachi S-3000 cluster/408 (2x4) (2 ns) & 8 & 54.1 & 31200 & 3760 & 64 \\
\hline $\begin{array}{l}\text { IBM eServer Cluster 1350-xSeries } 335 \text { w/2.8 GHz Xeon and } \\
\text { GigE }\end{array}$ & 16 & 54.05 & 38000 & 7600 & 89.6 \\
\hline Sun Fire E6900 (UltraSPARC IV 1.35 Ghz w/custom) & 24 & 53.81 & 101658 & 4500 & 64.8 \\
\hline IBM eServer p5 570 (1900 MHz POWER5) & 8 & 53.80 & 53600 & 10000 & 60.8 \\
\hline IBM Power 520 (4.2GHz, POWER6) & 4 & 53.6 & 47400 & 800 & 67 \\
\hline IBM Power 520 (4.2GHz POWER6) & 4 & 53.59 & 47400 & 800 & 67.2 \\
\hline SGI Origin $3000(500 \mathrm{MHz})$ & 64 & 53.16 & 81920 & 81920 & 64 \\
\hline CRAY T3E-1200E (600 MHz) & 64 & 53.07 & 62976 & 4992 & 76.8 \\
\hline Presto II PC cluster(PIII 824MHz w/fast enet) & 132 & 52.83 & 68520 & 68520 & 108.8 \\
\hline IBM S80s (450 MHz, SP switch) & 96 & 52.65 & 58000 & 13000 & 86.4 \\
\hline Sun Fire 12K (1050MHz/8MB E\$) & 32 & 52.58 & 66166 & 6000 & 67.2 \\
\hline Sun Fire $12 \mathrm{~K}$ (900MHz/8MB L2\$, perflib) & 36 & 52.05 & 48108 & 5500 & 64.8 \\
\hline
\end{tabular}




\begin{tabular}{|c|c|c|c|c|c|}
\hline $\begin{array}{c}\text { Computer } \\
\text { (Full Precision) }\end{array}$ & $\begin{array}{l}\text { Number } \\
\text { of Procs } \\
\text { or Cores }\end{array}$ & $\begin{array}{c}\boldsymbol{R}_{\max } \\
\text { GFlop/s }\end{array}$ & $\begin{array}{c}N_{\max } \\
\text { Order }\end{array}$ & $\begin{array}{c}N_{1 / 2} \\
\text { Order }\end{array}$ & $\begin{array}{c}\boldsymbol{R}_{\text {Peak }} \\
\text { GFlop/s }\end{array}$ \\
\hline IBM Power 520 (4.2 GHz POWER6 SLES 10 SP1) & 4 & 51.5 & 39840 & 2950 & 67.2 \\
\hline SGI Origin $2000(250 \mathrm{MHz})$ & 128 & 51.44 & 61000 & 10000 & 64 \\
\hline Cray T932 (2.2 ns) *** & 24 & 51.1 & 16384 & 1000 & 43 \\
\hline Sun Ultra HPC10000 Cluster/2(250 MHz,4MB L2) & 128 & 51.08 & 44352 & 12096 & 64 \\
\hline Cray T3D $512(150 \mathrm{MHz})$ & 512 & 50.8 & 57856 & 7136 & 76 \\
\hline HITACHI SR8000-G1/4(450MHz) & 4 & 50.59 & 31248 & 1704 & 57.6 \\
\hline Sun Fire 6900 (UltraSPARC IV, $1.2 \mathrm{GHz}$ ) & 24 & 49.64 & 96116 & 4100 & 57.6 \\
\hline IBM System p5 550Q (1.65GHz POWER5+) & 8 & 48.96 & 57200 & 840 & 52.8 \\
\hline Sun Ultra HPC10000 Cluster/4(250 MHz,4MB L2) & 128 & 48.85 & 80640 & 10368 & 64 \\
\hline LANL Avalon Cluster:Alpha $533 \mathrm{Mhz}+100 \mathrm{Mb} / \mathrm{s} \mathrm{sw}$ & 140 & 48.6 & 62720 & 25200 & 149.4 \\
\hline HP Integrity rx6600 (1.6GHz/24MB Dual-Core Itanium 2) & 8 & 48.55 & 47000 & 920 & 51.2 \\
\hline SGI2400(Origin 2000)Cluster(4x32 $300 \mathrm{MHz}$ ) & 128 & 48.33 & 57600 & 9500 & 76.8 \\
\hline Cray SV1ex-1-32, 500MHz & 32 & 48.17 & 40320 & 4150 & 64 \\
\hline Cray X-1 (800 MHz) & 4 & 47.8 & 41984 & 3456 & 51.2 \\
\hline HP 9000 rp7420-16 (1000MHz PA-8800) & 16 & 47.5 & 30600 & 1020 & 64 \\
\hline SGI Origin 200 (2x64 $300 \mathrm{MHz}$ w/fast enet) & 128 & 47.49 & 43000 & 86300 & 47.5 \\
\hline Intel Cluster PIII $500 \mathrm{MHz}$ quad w/Giganet+NT4 & 252 & 47.38 & 65520 & 98280 & 126 \\
\hline Hewlett-Packard V2600 (552 MHz) & 48 & 47.24 & 50040 & 9548 & 105.9 \\
\hline Compaq Alphaserver GS320 (1001Mhz 4MB L2) & 32 & 47.1 & 40000 & 5000 & 64.0 \\
\hline Hewlett-Packard SuperDome $552 \mathrm{MHz}$ & 32 & 47.01 & 41000 & 1472 & 70.7 \\
\hline IBM eServer pSeries 655/651(1.1GHz Power 4) & 16 & 46.92 & 75000 & 4000 & 70.4 \\
\hline Sun Fire $12 \mathrm{~K}(900 \mathrm{MHz} / 8 \mathrm{MB}$ L2\$, perflib) & 32 & 46.63 & 48108 & 5000 & 57.6 \\
\hline Sun Ultra HPC6000 Cluster/4(250 MHz,4MB L2) & 120 & 46.56 & 53760 & 24192 & 60 \\
\hline CRAY X1 (800 MHz, 4 procs) & 4 & 46.5 & 41984 & 3048 & 51.2 \\
\hline HP Integrity rx4640 (1.6GHz/24MB Dual-Core Itanium 2) & 8 & 46.31 & 49000 & 920 & 51.2 \\
\hline Cray SV1ex-1-32, 500MHz & 30 & 46.21 & 39690 & 4600 & 60 \\
\hline Fujitsu VPP500/32 (10nsec) & 32 & 46.1 & 29760 & 5350 & 51 \\
\hline Sun Fire 12K (1050MHz/8MB E\$) & 28 & 46.04 & 66166 & 5500 & 58.8 \\
\hline Fujitsu VPP700/22 (7nsec) & 22 & 45.9 & 67320 & 4840 & 48.4 \\
\hline SGI Origin $2000(300 \mathrm{Mhz})$ & 96 & 45.70 & 53248 & 8000 & 58 \\
\hline IBM System p5 550Q (1.5GHz POWER5+) & 8 & 44.68 & 65000 & 820 & 48.0 \\
\hline Sun HPC 10000(400MHz 8MB L2 Cache) & 64 & 44.57 & 39936 & 4032 & 51.2 \\
\hline HP Integrity rx7620 (1.5GHz Itanium2, 6MB L3 Cache) & 8 & 44.4 & 33000 & 1000 & 48.0 \\
\hline Cray SV1ex-1-32, 500MHz & 28 & 44.28 & 37044 & 4000 & 56 \\
\hline IBM SP2-T2 (66 MHz) & 256 & 44.2 & 53000 & 13500 & 68 \\
\hline HITACHI SR8000/6(250MHz) & 6 & 43.91 & 28000 & 2000 & 48 \\
\hline Sun HPC 10000(400MHz 4MB L2 Cache) & 64 & 43.82 & 39936 & 4032 & 51.2 \\
\hline SGI Origin $3000400 \mathrm{MHz}, 64 \mathrm{CPU}$ & 64 & 43.15 & 36864 & 36864 & 51.2 \\
\hline Cray SV1ex-1-32, 500MHz & 27 & 42.44 & 35721 & 4150 & 54 \\
\hline IBM SP 8 nodes (222 MHz POWER3) & 64 & 41.76 & 53000 & 10000 & 56.8 \\
\hline Sun E6000 "WildFire" 4 servers $(250 \mathrm{MHz})$ & 104 & 41.58 & 34944 & 9408 & 52 \\
\hline SGI Origin $2000400 \mathrm{MHz}, 64 \mathrm{CPU}$ & 64 & 41.53 & 81920 & 81920 & 51.2 \\
\hline CRAY T3E-900 (450 MHz) & 64 & 41.52 & 43776 & 4608 & 58 \\
\hline Fujitsu-Siemens hpcLine(Pentium II,450 MHz) & 192 & 41.45 & 56480 & 11136 & 86.4 \\
\hline Sun HPC $10000(400 \mathrm{MHz} 4 \mathrm{MB}$ L2 Cache) & 60 & 41.19 & 39936 & 3840 & 48.0 \\
\hline
\end{tabular}




\begin{tabular}{|c|c|c|c|c|c|}
\hline $\begin{array}{c}\text { Computer } \\
\text { (Full Precision) }\end{array}$ & $\begin{array}{l}\text { Number } \\
\text { of Procs } \\
\text { or Cores }\end{array}$ & $\begin{array}{c}\boldsymbol{R}_{\max } \\
\text { GFlop/s }\end{array}$ & $\begin{array}{c}N_{\max } \\
\text { Order }\end{array}$ & $\begin{array}{c}N_{1 / 2} \\
\text { Order }\end{array}$ & $\begin{array}{c}\boldsymbol{R}_{\text {Peak }} \\
\text { GFlop/s }\end{array}$ \\
\hline Sun Fire 12K (900MHz/8MB L2\$, perflib) & 28 & 40.95 & 48108 & 4200 & 50.4 \\
\hline Hitachi S-3000 cluster/306 (2x3) (2 ns) & 6 & 40.9 & 27000 & 2400 & 48 \\
\hline HITACHI SR8000-F1/4(375MHz) & 4 & 40.76 & 23000 & 1720 & 48 \\
\hline Hitachi S-3000 cluster/206 (3x2) (2 ns) & 6 & 40.6 & 21600 & 2160 & 48 \\
\hline Compaq ES40/EV6 AlphaServer SC & 64 & 40.3 & 57000 & & 64 \\
\hline SGI Origin $2000(195 \mathrm{MHz})$ & 128 & 40.25 & 60000 & 6000 & 49.9 \\
\hline IBM SP 32 nodes (200 MHz POWER3) & 64 & 39.90 & 63000 & 7400 & 51.2 \\
\hline Sun Fire $12 \mathrm{~K}(1050 \mathrm{MHz} / 8 \mathrm{MB} \mathrm{E} \$)$ & 24 & 39.65 & 66166 & 4500 & 50.4 \\
\hline SGI Origin2000 (8x16 $250 \mathrm{MHz}$ w/fast enet) & 128 & 39.40 & 82000 & 26000 & 64.0 \\
\hline Cray SV1ex-1-32, 500MHz & 25 & 39.09 & 34650 & 4150 & 50 \\
\hline Sun HPC 10000(400MHz 4MB L2 Cache) & 56 & 38.53 & 39936 & 3456 & 44.8 \\
\hline Sun Ultra HPC6000 Cluster/4(250 MHz,4MB L2) & 96 & 38.44 & 53760 & 19968 & 48 \\
\hline Cray SV1ex-1-32, 500MHz & 24 & 38.31 & 34776 & 3700 & 48 \\
\hline IBM S80s (450 MHz, SP switch) & 72 & 38.25 & 50000 & 11000 & 64.8 \\
\hline Sun E6000 "WildFire" 4 servers (250 MHz) & 96 & 38.13 & 29568 & 8064 & 48 \\
\hline Sun Ultra HPC10000 Cluster/2(250 MHz,4MB L2) & 96 & 37.79 & 50400 & 6528 & 48 \\
\hline Fujitsu VPP5000/4 (3.33nsec) & 4 & 37.60 & 60384 & 1584 & 38.4 \\
\hline SGI Origin 2000 Ether Cluster(250 MHz,4x32) & 128 & 37.31 & 56000 & 23000 & 64 \\
\hline IBM eServer pSeries $655(1.7 \mathrm{GHz}$ POWER4+) & 8 & 37.29 & 55000 & 600 & 54.4 \\
\hline Sun Ultra HPC10000 Cluster/3(250 MHz,4MB L2) & 96 & 36.91 & 65520 & 8640 & 48 \\
\hline Compaq GS140 cluster & 64 & 36.70 & 40932 & 5200 & 67 \\
\hline Cray T932 (2.2 ns) *** & 16 & 36.6 & 16384 & 1000 & 29 \\
\hline Fujitsu VPP300/16E (6.5nsec) & 16 & 36.4 & 57600 & 3520 & 38 \\
\hline Fujitsu VPP700/16E (6.5nsec) & 16 & 36.4 & 57600 & 3520 & 38 \\
\hline IBM SP 2 nodes (375 MHz POWER3 High) & 32 & 36.27 & 38000 & 7200 & 48.0 \\
\hline Sun Fire E6900 (UltraSPARC IV 1.35 Ghz w/custom) & 16 & 36.16 & 84155 & 3600 & 43.2 \\
\hline Hewlett-Packard V2600 (550 MHz) & 32 & 36.01 & 41000 & 5040 & 70.4 \\
\hline Sun HPC 10000(400MHz 4MB L2 Cache) & 52 & 35.83 & 39936 & 3264 & 41.6 \\
\hline Sun Fire 6800 (900MHz/8MB L2) & 24 & 35.63 & 48108 & 5000 & 43.2 \\
\hline HP AlphaServer GS1280 7/1300 (1.3 GHz) & 16 & 35.6 & 40000 & & 41.6 \\
\hline HITACHI SR8000-E1/4(300MHz) & 4 & 35.57 & 31248 & 1600 & 38.4 \\
\hline Sun Fire 12K (900MHz/8MB L2\$, perflib) & 24 & 35.06 & 48108 & 3700 & 43.2 \\
\hline IBM SP 8 nodes (375 MHz POWER3 Thin) & 32 & 34.51 & 53000 & 7000 & 48 \\
\hline NEC SX-4/16 $(8.0 \mathrm{~ns}) * * *$ & 16 & 34.42 & 14336 & 960 & 32 \\
\hline Parnass2 Cluster (PII 400 MHz w/Myricon) & 144 & 34.23 & 64224 & 7200 & 57.6 \\
\hline Sun HPC 10000(333MHz 4MB L2 Cache) & 64 & 34.17 & 20352 & 3648 & 42.6 \\
\hline Fujitsu VPP300/16 ( 7nsec) & 16 & 34.1 & 59200 & 3520 & 35 \\
\hline Fujitsu VPP700/16 ( 7nsec) & 16 & 34.1 & 59200 & 3520 & 35 \\
\hline Sun HPC 10000(400MHz 8MB L2 Cache) & 48 & 33.85 & 39936 & 3072 & 38.4 \\
\hline IBM eServer BladeCenter JS2 1 (2.5 GHz Power PC) & 4 & 33.72 & 30800 & 3700 & 40.0 \\
\hline Compaq GS140 cluster & 56 & 33.70 & 40932 & 4588 & 58 \\
\hline SGI Origin2000 (6x16 $300 \mathrm{MHz}$ w/fast enet) & 96 & 33.61 & 71500 & 21000 & 57.6 \\
\hline Compaq Alphaserver GS320 (731Mhz 4MB L2) & 32 & 33.54 & 40000 & 4700 & 46.8 \\
\hline Sun HPC 10000(400MHz 4MB L2 Cache) & 48 & 33.09 & 39936 & 3072 & 38.4 \\
\hline
\end{tabular}




\begin{tabular}{|c|c|c|c|c|c|}
\hline $\begin{array}{c}\text { Computer } \\
\text { (Full Precision) }\end{array}$ & $\begin{array}{l}\text { Number } \\
\text { of Procs } \\
\text { or Cores }\end{array}$ & $\begin{array}{c}\boldsymbol{R}_{\max } \\
\text { GFlop/s }\end{array}$ & $\begin{array}{c}N_{\max } \\
\text { Order }\end{array}$ & $\begin{array}{c}N_{1 / 2} \\
\text { Order }\end{array}$ & $\begin{array}{c}\boldsymbol{R}_{\text {Peak }} \\
\text { GFlop/s }\end{array}$ \\
\hline Sun Fire 12K (1050MHz/8MB E\$) & 20 & 33.08 & 66166 & 4500 & 42.0 \\
\hline Sun Fire 6900 (UltraSPARC IV, 1.2 GHz) & 16 & 32.88 & 66166 & 320 & 38.4 \\
\hline IBM eServer pSeries 655 (1.5GHz POWER4+) & 8 & 32.59 & 55000 & 600 & 48.0 \\
\hline NEC Express5800/1160Xa (800MHz) & 16 & 32.29 & 62504 & 7000 & 51.2 \\
\hline SGI Origin2000 (300 Mhz) & 64 & 32.29 & 81976 & 12324 & 38.4 \\
\hline Sun HPC $10000(333 \mathrm{MHz} 4 \mathrm{MB}$ L2 Cache) & 60 & 32.27 & 20352 & 3456 & 40.0 \\
\hline IBM BladeCenter JS21 dual-core PowerPC 970MP, $2.5 \mathrm{GHz}$ & 4 & 32.22 & 39100 & 2052 & 40.0 \\
\hline Cray SV1ex-1-32, 500MHz & 20 & 32.11 & 32760 & 3350 & 40 \\
\hline Intel Core 2 Q6600 Kensfield) (4 core, $2.4 \mathrm{GHz}$ ) & 4 & 31.90 & 15000 & 1664 & 38.4 \\
\hline SGI Origin2000 (3x32 $250 \mathrm{MHz}$ w/fast enet) & 96 & 31.84 & 58000 & 20000 & 48.0 \\
\hline NEC SX-8/2 (2 GHz) & 2 & 31.72 & 30720 & & 32 \\
\hline Paragon XP/S MP(256 Nodes, OS=SUNMOS S1.6) & 768 & 31.7 & 43500 & 8400 & 38 \\
\hline HP V2500 (32 proc. $440 \mathrm{MHz})$ & 32 & 31.59 & 41000 & 4720 & 56.3 \\
\hline IBM System p5 550 (2.1GHz POWER5+) & 4 & 31.50 & 53100 & 500 & 33.6 \\
\hline hp AlphaServer GS1280 7/1150(1.15 GHz) & 16 & 31.46 & 40000 & & 36.8 \\
\hline SGI Origin 2000 Ether Cluster(195 MHz,4x32) & 128 & 31.36 & 56000 & 21000 & 50 \\
\hline NEC SX-4/16 (8.0 ns) & 16 & 31.10 & 20480 & 960 & 32 \\
\hline NEC SX-4/16M2 (8.0 ns) & 16 & 31.09 & 20480 & 204832 & \\
\hline Sun HPC 6500 Cluster/4 (250 MHz, 4MB L2) & 80 & 30.98 & 24192 & 13440 & 40 \\
\hline DEC AlphaServer 8400 5/612 (625 MHz) & 64 & 30.90 & 30704 & 8360 & 80 \\
\hline NEC SX-4/16A (8.0 ns) & 16 & 30.83 & 20480 & 960 & 32 \\
\hline Cray SV1-1-32 (300 MHz) & 32 & 30.72 & 40320 & 4150 & 39.2 \\
\hline SGI Origin 2000 Ether Cluster(250 MHz,3x32) & 96 & 30.70 & 49000 & 17000 & 48 \\
\hline Thinking Machines CM-5 & 512 & 30.4 & 36864 & 16384 & 66 \\
\hline Sun HPC 10000(400MHz 4MB L2 Cache) & 44 & 30.33 & 39936 & 2688 & 35.2 \\
\hline Sun HPC 10000(333MHz 4MB L2 Cache) & 56 & 30.27 & 20352 & 3264 & 37.3 \\
\hline $\begin{array}{l}\text { ClearSpeed CSX600 Advance accelerator boards }(250 \mathrm{MHz}) \\
\text { (frontend IBM Intellistation (dual Opteron } 2502.4 \mathrm{GHz} \\
\text { PCI-X board) }\end{array}$ & 3 & 30.2 & 20256 & 4712 & 100.8 \\
\hline Cray SV1-1-32 (300 MHz) & 30 & 30.04 & 39690 & 4600 & 36 \\
\hline Sun Fire $6800(750 \mathrm{MHz} / 8 \mathrm{MB} \mathrm{L} 2 \$)$ & 24 & 29.65 & 48108 & & 36 \\
\hline Hitachi SR2201/128(150MHz) & 128 & 29.46 & 51840 & 7680 & 38.4 \\
\hline IBM SP2 (160 MHz) & 64 & 29.45 & 27500 & 5700 & 41 \\
\hline Sun Fire $12 \mathrm{~K}(900 \mathrm{MHz} / 8 \mathrm{MB}$ L2\$, perflib) & 20 & 29.30 & 48108 & 3300 & 36.0 \\
\hline HITACHI SR8000/4(250MHz) & 4 & 29.1 & 21464 & 1600 & 32 \\
\hline IBM SP2-T2 (66 MHz) & 160 & 28.7 & 42200 & 10300 & 42 \\
\hline Compaq GS140 cluster & 48 & 28.58 & 40932 & 4200 & 50 \\
\hline Cray T3E-1350 (675 MHz) & 32 & 28.5 & 44544 & 3456 & 43.2 \\
\hline IBM System p5 550 (1.9GHz POWER5+) & 4 & 28.49 & 53100 & 250 & 30.0 \\
\hline IBM eServer pSeries 650 6M2(1.45GHz POWER4+) & 8 & 28.41 & 60000 & 600 & 46.4 \\
\hline Hitachi S-3800/480 (2 ns) & 4 & 28.4 & 15500 & 830 & 32 \\
\hline Sun HPC 10000(333MHz 4MB L2 Cache) & 52 & 28.32 & 20352 & 3072 & 34.6 \\
\hline CRAY T3E (300 MHz) & 64 & 28.31 & 29952 & 4032 & 38.4 \\
\hline IBM SP 16 nodes (332 MHz 604e) & 64 & 28.12 & 36000 & 6760 & 42 \\
\hline
\end{tabular}




\begin{tabular}{|c|c|c|c|c|c|}
\hline $\begin{array}{c}\text { Computer } \\
\text { (Full Precision) }\end{array}$ & $\begin{array}{l}\text { Number } \\
\text { of Procs } \\
\text { or Cores }\end{array}$ & $\begin{array}{c}\boldsymbol{R}_{\max } \\
\text { GFlop/s }\end{array}$ & $\begin{array}{c}N_{\max } \\
\text { Order }\end{array}$ & $\begin{array}{c}N_{1 / 2} \\
\text { Order }\end{array}$ & $\begin{array}{c}\boldsymbol{R}_{\text {Peak }} \\
\text { GFlop/s }\end{array}$ \\
\hline Cray SV1-1-32 (300 MHz) & 28 & 28.01 & 37044 & 4000 & 33.6 \\
\hline Hitachi S-3000 cluster/204 (2x2) (2 ns) & 4 & 27.9 & 21600 & 1640 & 32 \\
\hline HP Exemplar X-Class SPP-UX 5.2 & 64 & 27.56 & 29956 & 4584 & 46 \\
\hline Sun HPC 10000(400MHz 4MB L2 Cache) & 40 & 27.56 & 39936 & 2496 & 32.0 \\
\hline IBM eServer p5 570 (1900 MHz POWER5) & 4 & 27.52 & 38000 & 1400 & 30.40 \\
\hline IBM S80s (450 MHz, SP switch) & 48 & 27.28 & 41000 & 9000 & 43.2 \\
\hline Hitachi S-3000 cluster/404 (1x4) (2 ns) & 4 & 27.2 & 31200 & 2680 & 32 \\
\hline CRAY SV1-1-32 (300 MHz) & 27 & 26.82 & 35721 & 4150 & 32 \\
\hline SGI POWER CHALLENGE (90 MHz) & 128 & 26.7 & 53000 & 20000 & 46 \\
\hline CRAY T3E-1200E (600 MHz) & 32 & 26.58 & 44544 & 3456 & 38.4 \\
\hline Sun Fire $12 \mathrm{~K}(1050 \mathrm{MHz} / 8 \mathrm{MB} \mathrm{E} \$)$ & 16 & 26.57 & 66166 & 3500 & 33.6 \\
\hline Sun Ultra HPC 10000(250 MHz 4MB L2 Cache) & 64 & 26.45 & 19968 & 3072 & 32 \\
\hline Sun HPC 10000(333MHz 4MB L2 Cache) & 48 & 26.38 & 20352 & 2880 & 32.0 \\
\hline SGI Origin $2000(250 \mathrm{MHz})$ & 64 & 26.24 & 43520 & 5200 & 32 \\
\hline HITACHI SR8000-G1/2(450MHz) & 2 & 25.55 & 23000 & 1256 & 28.8 \\
\hline Sun HPC 6500 Cluster/4 (250 MHz, 4MB L2) & 64 & 25.40 & 26880 & 10752 & 32 \\
\hline DEC AlphaServer 8400 5/612 (625 MHz) & 56 & 25.39 & 26864 & 8360 & 70 \\
\hline Cray T3D $256(150 \mathrm{MHz})$ & 256 & 25.3 & 40960 & 4918 & 38 \\
\hline Compaq GS140 cluster & 40 & 25.17 & 40932 & 3824 & 42 \\
\hline CRAY SV1-1-32 (300 MHz) & 25 & 25.02 & 34650 & 4150 & 30 \\
\hline HP Integrity rx4640-8 (1.6GHz/9MB Itanium 2) & 4 & 24.49 & 38680 & 560 & 25.6 \\
\hline Sun Ultra HPC 10000(250 MHz 4MB L2 Cache) & 60 & 24.83 & 19968 & 2800 & 30 \\
\hline Sun HPC 10000(400MHz 4MB L2 Cache) & 36 & 24.77 & 39936 & 2304 & 28.8 \\
\hline Sun Fire $6800(750 \mathrm{MHz} / 8 \mathrm{MB}$ L2\$) & 20 & 24.71 & 48108 & & 30 \\
\hline DEC 8400 5/440 (440 MHz) & 64 & 24.7 & 30712 & 4584 & 56.3 \\
\hline $\begin{array}{l}\text { IBM BladeCenter JS12 Express ( } 3.8 \text { GHz POWER6 RHEL } \\
\text { 5.1) }\end{array}$ & 2 & 24.67 & 55000 & 2500 & 30.4 \\
\hline HP V2500 (24 proc. $440 \mathrm{MHz})$ & 24 & 24.64 & 41000 & 3120 & 42.2 \\
\hline HP Integrity rx3600 (1.6GHz/18MB Dual-Core Itanium 2) & 4 & 24.61 & 39480 & 560 & 25.6 \\
\hline SGI Origin 2000 Ether Cluster(195 MHz,3x32) & 96 & 24.58 & 49000 & 15000 & 37 \\
\hline HP Integrity rx2660 (1.6GHz/18MB Dual-Core Itanium 2) & 4 & 24.54 & 38760 & 560 & 25.6 \\
\hline HP Integrity BL860c (1.6GHz/18MB Dual-Core Itanium 2) & 4 & 24.48 & 34920 & 560 & 25.6 \\
\hline Sun HPC 10000(333MHz 4MB L2 Cache) & 44 & 24.36 & 20352 & 2688 & 29.3 \\
\hline HP Integrity rx2620 (1.6GHz/18MB Dual Core Itanium 2$)$ & 4 & 24.22 & 38000 & 560 & 25.6 \\
\hline Cray SV1ex-1-32, 500MHz & 16 & 24.22 & 30240 & 2950 & 32 \\
\hline IBM eServer OpenPower 720 (1.65GHz POWER5) & 4 & 24.12 & 63000 & 1500 & 26.40 \\
\hline Sun Fire $6800(900 \mathrm{MHz} / 8 \mathrm{MB} \mathrm{L} 2)$ & 16 & 24.12 & 48108 & 3500 & 28.8 \\
\hline Cray SV1-1-32 (300 MHz) & 24 & 24.03 & 34776 & 3700 & 28.8 \\
\hline Fujitsu VPP500/16 (10nsec) & 16 & 23.6 & 21120 & 3360 & 26 \\
\hline SGI Origin $300(500 \mathrm{MHz})$ & 32 & 23.59 & 29000 & 29000 & 32 \\
\hline IBM eServer p5 550 (1650 MHz POWER5) & 4 & 23.57 & 62000 & 1600 & 26.4 \\
\hline Sun Fire $12 \mathrm{~K}$ (900MHz/8MB L2\$, perflib) & 16 & 23.48 & 48108 & 2800 & 28.8 \\
\hline IBM eServer pSeries 655/651(1.1GHz Power 4) & 8 & 23.47 & 53000 & 600 & 35.2 \\
\hline IBM SP2 thin-node2,SP-sw,256MB/node(66 MHz) & 128 & 23.45 & 56000 & 9200 & 33.6 \\
\hline Sun Ultra HPC 10000(250 MHz 4MB L2 Cache) & 56 & 23.38 & 19968 & 2880 & 28 \\
\hline
\end{tabular}




\begin{tabular}{|c|c|c|c|c|c|}
\hline $\begin{array}{c}\text { Computer } \\
\text { (Full Precision) }\end{array}$ & $\begin{array}{l}\text { Number } \\
\text { of Procs } \\
\text { or Cores }\end{array}$ & $\begin{array}{c}\boldsymbol{R}_{\max } \\
\text { GFlop/s }\end{array}$ & $\begin{array}{c}N_{\max } \\
\text { Order }\end{array}$ & $\begin{array}{c}N_{1 / 2} \\
\text { Order }\end{array}$ & $\begin{array}{c}\boldsymbol{R}_{\text {Peak }} \\
\text { GFlop/s }\end{array}$ \\
\hline NEC SX-3/44R (2.5 ns) & 4 & 23.2 & 6400 & 830 & 26 \\
\hline IBM SP2-T2 (66 MHz) & 128 & 22.9 & 37000 & 9200 & 34 \\
\hline Sun HPC 10000(400MHz 8MB L2 Cache) & 32 & 22.63 & 39936 & 2112 & 25.6 \\
\hline IBM POWER2 Super Chip RS/6000 SP(120 MHz) & 64 & 22.55 & 27400 & 6500 & 31 \\
\hline IBM eServer pSeries 655 651(1.1GHz POWER4) & 8 & 22.34 & 36000 & 600 & 35.2 \\
\hline Sun HPC 10000(333MHz 4MB L2 Cache) & 40 & 22.27 & 20352 & 2496 & 26.6 \\
\hline Sun HPC 10000(400MHz 4MB L2 Cache) & 32 & 21.98 & 39936 & 2112 & 25.6 \\
\hline HP Integrity rx3600 (1.4GHz/12MB Dual-Core Itanium 2) & 4 & 21.83 & 39000 & 560 & 25.6 \\
\hline DEC 8400 5/440 (440 MHz) & 56 & 21.8 & 26856 & 4072 & 49.3 \\
\hline HP Integrity Server rx5670 (1500MHz, 6.0MB L3 Cache) & 4 & 21.713 & 51040 & 500 & 24.0 \\
\hline Sun Ultra HPC 10000(250 MHz 4MB L2 Cache) & 52 & 21.68 & 19968 & 2496 & 26 \\
\hline IBM eServer p5 550 Express (1500 MHz POWER5) & 4 & 21.64 & 53000 & 500 & 24.0 \\
\hline Sun HPC 6500(400MHz 8MB L2 Cache) & 30 & 21.61 & 39936 & 2688 & 24.0 \\
\hline Hitachi S-3800/380 (2 ns) & 3 & 21.6 & 15680 & 760 & 24 \\
\hline Hitachi S-3000 cluster/303 (1x3) (2 ns) & 3 & 21.5 & 27000 & 1560 & 24 \\
\hline HP Integrity rx2620 (1.4GHz/12MB Dual Core Itanium 2) & 4 & 21.41 & 36760 & 560 & 25.6 \\
\hline Sun Ultra HPC 10000(250 MHz 1MB L2 Cache) & 64 & 21.37 & 15000 & 4200 & 32.0 \\
\hline Sun Ultra HPC 10000(250 MHz 1MB L2 Cache) & 63 & 21.14 & 15000 & 4200 & 31.5 \\
\hline SGI Origin 2000 Ether Cluster(250 MHz,2x32) & 64 & 21.05 & 40000 & 14000 & 32 \\
\hline Sun Ultra HPC 10000(250 MHz 4MB L2 Cache) & 50 & 21.05 & 19968 & 2496 & 25 \\
\hline IBM SP 4 nodes (222 MHz POWER3) & 32 & 21.00 & 38000 & 5200 & 28.4 \\
\hline CRAY T3E-900 (450 MHz) & 32 & 20.86 & 31104 & 3072 & 29 \\
\hline SGI Origin $2000(195 \mathrm{MHz})$ & 64 & 20.75 & 43520 & 4608 & 25.0 \\
\hline Cray C90 $(240 \mathrm{MHz})^{* * *}$ & 16 & 20.65 & 13312 & 700 & 15 \\
\hline DEC AlphaServer 8400 5/612 (625 MHz) & 40 & 20.54 & 24552 & 8960 & 50 \\
\hline HITACHI SR8000-F1/2(375MHz) & 2 & 20.50 & 15176 & 1208 & 24 \\
\hline Hewlett-Packard V2600 (550 MHz) & 16 & 20.45 & 41000 & 2040 & 35.2 \\
\hline Sun Ultra HPC 10000(250 MHz 1MB L2 Cache) & 60 & 20.31 & 15000 & 3600 & 30.0 \\
\hline Sun Ultra HPC 10000(250 MHz 4MB L2 Cache) & 48 & 20.30 & 19968 & 2496 & 24 \\
\hline Compaq GS140 cluster & 32 & 20.22 & 30712 & 3056 & 34 \\
\hline Sun HPC 6500(400MHz 8MB L2 Cache) & 28 & 20.20 & 39936 & 2496 & 22.4 \\
\hline Cray SV1-1-32 (300 MHz) & 20 & 20.18 & 32760 & 3350 & 24 \\
\hline Sun HPC 10000(333MHz 4MB L2 Cache) & 36 & 20.11 & 20352 & 2304 & 24.0 \\
\hline NEC SX-3/44 (2.9 ns) & 4 & 20.0 & 6144 & 832 & 22 \\
\hline IBM SP 16 nodes (200 MHz POWER3) & 32 & 19.92 & 44800 & 4750 & 25.6 \\
\hline Sun Fire 6800 (750MHz/8MB L2\$) & 16 & 19.90 & 48108 & & 24 \\
\hline Sun HPC 6500 Cluster/2 (250 MHz, 4MB L2) & 48 & 19.42 & 18816 & 5376 & 24 \\
\hline LANL Avalon Cluster:Alpha $533 \mathrm{Mhz}+100 \mathrm{Mb} / \mathrm{s} \mathrm{sw}$ & 68 & 19.33 & 30464 & 14376 & 72.5 \\
\hline Cray SV1ex-1-32, 500MHz & 12 & 19.26 & 25704 & 2700 & 24 \\
\hline DEC 8400 5/440 (440 MHz) & 48 & 19.2 & 23032 & 4048 & 42.2 \\
\hline Sun HPC 10000(400MHz 4MB L2 Cache) & 28 & 19.16 & 39936 & 1920 & 22.4 \\
\hline Sun Ultra HPC 10000(250 MHz 1MB L2 Cache) & 56 & 19.14 & 15000 & 3600 & 28.0 \\
\hline IBM eServer pSeries 655 (1.7GHz POWER4+) & 4 & 18.99 & 38000 & 400 & 27.2 \\
\hline IBM eServer BladeCenter JS21 (2.7 GHz Power PC) & 2 & 18.96 & 30800 & 2500 & 21.6 \\
\hline
\end{tabular}




\begin{tabular}{|c|c|c|c|c|c|}
\hline $\begin{array}{c}\text { Computer } \\
\text { (Full Precision) }\end{array}$ & $\begin{array}{l}\text { Number } \\
\text { of Procs } \\
\text { or Cores }\end{array}$ & $\begin{array}{c}\boldsymbol{R}_{\max } \\
\text { GFlop/s }\end{array}$ & $\begin{array}{l}N_{\max } \\
\text { Order }\end{array}$ & $\begin{array}{c}N_{1 / 2} \\
\text { Order }\end{array}$ & $\begin{array}{c}\boldsymbol{R}_{\text {Peak }} \\
\text { GFlop/s }\end{array}$ \\
\hline Fujitsu VPP5000/2 (3.33nsec) & 2 & 18.82 & 42720 & 1056 & 19.2 \\
\hline Sun HPC 6500(400MHz 8MB L2 Cache) & 26 & 18.78 & 39936 & 2304 & 20.8 \\
\hline Sun Ultra HPC 10000(250 MHz 4MB L2 Cache) & 44 & 18.67 & 19968 & 2496 & 22 \\
\hline Fujitsu VPP300/8E (6.5nsec) & 8 & 18.6 & 41600 & 2400 & 19 \\
\hline Fujitsu VPP700/8E (6.5nsec) & 8 & 18.6 & 41600 & 2400 & 19 \\
\hline SGI POWER CHALLENGE (75 MHz) & 96 & 18.5 & 53000 & 20000 & 29 \\
\hline Thinking Machines CM-200 (half precision) & 2048 & 18.5 & 39936 & 14336 & 40 \\
\hline IBM SP 1 node (375 MHz POWER3 High) & 16 & 18.25 & 27000 & 1300 & 24.0 \\
\hline Sun Fire $6800(900 \mathrm{MHz} / 8 \mathrm{MB}$ L2) & 12 & 18.17 & 48108 & 2680 & 21.6 \\
\hline Sun Fire E6900 (UltraSPARC IV 1.35 Ghz w/custom) & 8 & 17.98 & 60118 & 2200 & 21.6 \\
\hline DEC AlphaServer 8400 5/612 (625 MHz) & 32 & 17.96 & 25624 & 4088 & 40 \\
\hline HP AlphaServer GS1280 7/1300 (1.3 GHz) & 8 & 17.93 & 32768 & & 20.8 \\
\hline Sun HPC $10000(333 \mathrm{MHz} 4 \mathrm{MB}$ L2 Cache) & 32 & 17.91 & 20352 & 2112 & 21.3 \\
\hline Sun HPC 6000(336MHz 4MB L2 Cache) & 30 & 17.89 & 20352 & 2112 & 20.2 \\
\hline IBM SP 4 nodes (375 MHz POWER3 Thin) & 16 & 17.66 & 38000 & 3300 & 24 \\
\hline IBM BladeCenter JS21 dual-core PowerPC 970MP, $2.7 \mathrm{GHz}$ & 2 & 17.65 & 41000 & 600 & 21.6 \\
\hline IBM Power 570 (5.0 GHz POWER6) & 1 & 17.47 & 20000 & 280 & 20.0 \\
\hline HP V2500 (16 proc. $440 \mathrm{MHz})$ & 16 & 17.47 & 41000 & 1580 & 28.2 \\
\hline SGI Origin 2000 Ether Cluster(195 MHz,2x32) & 64 & 17.46 & 40000 & 13000 & 25 \\
\hline NEC SX-3/34R (2.5 ns) & 3 & 17.4 & 6144 & 691 & 19 \\
\hline Sun HPC 6500(400MHz 8MB L2 Cache) & 24 & 17.35 & 39936 & 2112 & 19.2 \\
\hline Intel Core 2 Q6600 Kensfield) (2 core, $2.4 \mathrm{GHz}$ ) & 2 & 17.25 & 15000 & 1664 & 19.2 \\
\hline Sun Ultra HPC 10000(250 MHz 4MB L2 Cache) & 40 & 17.12 & 19968 & 2496 & 20 \\
\hline Fujitsu VPP300/8 ( 7nsec) & 8 & 17.1 & 41600 & 2080 & 18 \\
\hline Fujitsu VPP700/8 ( 7nsec) & 8 & 17.1 & 41600 & 2080 & 18 \\
\hline Sun HPC 6000(336MHz 4MB L2 Cache) & 28 & 16.74 & 20352 & 2112 & 18.8 \\
\hline Sun Ultra2/2200 Sparc Cluster & 32 & 16.71 & 28416 & 9216 & 25.6 \\
\hline DEC 8400 5/440 (440 MHz) & 40 & 16.7 & 20456 & 3200 & 35.2 \\
\hline Sun Ultra HPC 10000(250 MHz 1MB L2 Cache) & 48 & 16.66 & 15000 & 3600 & 24.0 \\
\hline IBM Power 595 (5.0 GHz POWER6) & 1 & 16.4 & 22900 & 300 & 20 \\
\hline Sun HPC 10000(400MHz 4MB L2 Cache) & 24 & 16.39 & 39936 & 1728 & 19.2 \\
\hline Sun Fire 6900 (UltraSPARC IV, $1.2 \mathrm{GHz}$ ) & 8 & 16.36 & 48108 & 220 & 19.2 \\
\hline Cray SV1-1-32 (300 MHz) & 16 & 16.23 & 30240 & 2950 & 19.6 \\
\hline Paragon XP/S MP(128 Nodes, OS=SUNMOS S1.6) & 384 & 16.0 & 30700 & 5700 & 19 \\
\hline Cray C90 $(240 \mathrm{MHz})^{* * *}$ & 12 & 15.97 & 13312 & 600 & 12 \\
\hline Sun HPC 4500 Cluster/4 (250 MHz, 4MB L2) & 44 & 15.96 & 26880 & 8064 & 22 \\
\hline Sun HPC 6500(400MHz 8MB L2 Cache) & 22 & 15.92 & 39936 & 1920 & 17.6 \\
\hline IBM IntelliStation POWER 285 (2.1 GHz Power5+) & 2 & 15.88 & 41100 & 310 & 16.8 \\
\hline NEC SX-8/1 (2 GHz) & 1 & 15.87 & 30720 & & 16 \\
\hline SGI Origin $2000(300 \mathrm{Mhz})$ & 32 & 15.77 & 30720 & 4500 & 19 \\
\hline hp AlphaServer GS1280 7/1150(1.15 GHz) & 8 & 15.72 & 30000 & & 18.4 \\
\hline Sun HPC 10000(333MHz 4MB L2 Cache) & 28 & 15.66 & 20352 & 1728 & 18.6 \\
\hline HP AlphaServer ES80 7/1150 (1.15 GHz) & 8 & 15.62 & 30000 & & 18.4 \\
\hline SGI POWER CHALLENGE (90 MHz) & 64 & 15.6 & 37000 & 8500 & 23 \\
\hline
\end{tabular}




\begin{tabular}{|c|c|c|c|c|c|}
\hline $\begin{array}{c}\text { Computer } \\
\text { (Full Precision) }\end{array}$ & $\begin{array}{l}\text { Number } \\
\text { of Procs } \\
\text { or Cores }\end{array}$ & $\begin{array}{c}\boldsymbol{R}_{\max } \\
\text { GFlop/s }\end{array}$ & $\begin{array}{c}N_{\max } \\
\text { Order }\end{array}$ & $\begin{array}{c}N_{1 / 2} \\
\text { Order }\end{array}$ & $\begin{array}{c}\boldsymbol{R}_{\text {Peak }} \\
\text { GFlop/s }\end{array}$ \\
\hline Sun HPC 6000(336MHz 4MB L2 Cache) & 26 & 15.59 & 20352 & 1920 & 17.5 \\
\hline IBM Power 570 (4.7GHz POWER6) & 1 & 15.53 & 26600 & 280 & 18.8 \\
\hline NEC SX-4/8M2 (8.0 ns) & 8 & 15.44 & 9984 & 192016 & \\
\hline NEC SX-4/8 (8.0 ns) & 8 & 15.43 & 9984 & 860 & 16 \\
\hline Sun Ultra HPC 10000(250 MHz 4MB L2 Cache) & 36 & 15.42 & 19968 & 2112 & 18 \\
\hline IBM eServer pSeries 630 6C4 (1.45GHz POWER4+) & 4 & 15.34 & 38000 & 400 & 23.2 \\
\hline IBM eServer pSeries 630 6E4 (1.45GHz POWER4+) & 4 & 15.34 & 38000 & 400 & 23.2 \\
\hline Compaq GS140 cluster & 24 & 15.31 & 30712 & 2200 & 25 \\
\hline NEC SX-4/8A (8.0 ns) & 8 & 15.31 & 9984 & 860 & 16 \\
\hline IBM IntelliStation POWER 185 (2.5GHz) & 2 & 15.28 & 29000 & 1400 & 20.0 \\
\hline IBM System p5 185 (2.5GHz) & 2 & 15.28 & 29000 & 1400 & 20.0 \\
\hline Intel Paragon XPS-35 (50 MHz, OS=R1.1) & 512 & 15.2 & 23000 & 9000 & 26 \\
\hline IBM S80 (450 MHz) & 24 & 15.17 & 29000 & 4400 & 21.6 \\
\hline hp server rx5670 (1000MHz, 3.0MB L3 Cache) & 4 & 15.13 & 37920 & 1440 & 16 \\
\hline Thinking Machines CM-5 & 256 & 15.1 & 26112 & 12032 & 33 \\
\hline HP Exemplar X-Class SPP-UX 5.2 & 32 & 15.01 & 26848 & 1840 & 23 \\
\hline IBM Power 575 (4.7 GHz POWER6) & 1 & 15.0 & 19500 & 300 & 19 \\
\hline Sun Fire 6800 (750MHz/8MB L2\$) & 12 & 14.96 & 48108 & & 18 \\
\hline IBM SP2 (160 MHz) & 32 & 14.93 & 20000 & 3840 & 20 \\
\hline HITACHI SR2201/64(150MHz) & 64 & 14.89 & 38880 & 6720 & 19 \\
\hline Hitachi S-3800/280 (2 ns) & 2 & 14.6 & 15680 & 570 & 16 \\
\hline HITACHI SR8000/2(250MHz) & 2 & 14.6 & 15176 & 1192 & 16 \\
\hline IBM Power 570 (4.2 GHz POWER6) & 1 & 14.57 & 20000 & 360 & 16.8 \\
\hline Sun HPC 10000(333MHz 4MB L2 Cache) & 26 & 14.53 & 20352 & 1728 & 17.3 \\
\hline Hitachi S-3000 cluster/202 (1x2) (2 ns) & 2 & 14.5 & 21600 & 1100 & 16 \\
\hline IBM SP2 (77 MHz, switch of 4/96) & 64 & 14.5 & 27000 & 5100 & 20 \\
\hline Sun HPC 6000(336MHz 4MB L2 Cache) & 24 & 14.49 & 20352 & 1728 & 16.1 \\
\hline Sun HPC 6500(400MHz 8MB L2 Cache) & 20 & 14.49 & 39936 & 1728 & 16.0 \\
\hline IBM eServer pSeries 650 6M2(1.45GHz POWER4+) & 4 & 14.48 & 36000 & 400 & 23.2 \\
\hline Cray T3E-1350 (675 MHz) & 16 & 14.4 & 31680 & 2352 & 21.6 \\
\hline $\begin{array}{l}\text { IBM IntelliStation POWER } 285 \text { Workstation }(1.9 \mathrm{GHz} \\
\text { POWER5+) }\end{array}$ & 2 & 14.35 & 41200 & 300 & 15.2 \\
\hline IBM System p5 505 (1.9 GHz POWER5+) & 2 & 14.31 & 41200 & 300 & 15.2 \\
\hline Sun Ultra HPC 10000(250 MHz 1MB L2 Cache) & 40 & 14.06 & 15000 & 3000 & 20.0 \\
\hline CRAY T3E (300 MHz) & 32 & 14.03 & 21120 & 2832 & 19.2 \\
\hline Intel Delta (40 MHz) & 512 & 13.9 & 25000 & 7500 & 20 \\
\hline DEC AlphaServer 8400 5/612 (625 MHz) & 24 & 13.79 & 25624 & 3072 & 30 \\
\hline Sun Ultra HPC 10000(250 MHz 4MB L2 Cache) & 32 & 13.77 & 19968 & 1920 & 16 \\
\hline Sun HPC 10000(400MHz 4MB L2 Cache) & 20 & 13.74 & 39936 & 1536 & 16.0 \\
\hline Cray Y-MP C90 (240 MHz 4.2 ns) & 16 & 13.7 & 10000 & 650 & 15 \\
\hline DEC 8400 5/440 (440 MHz) & 32 & 13.7 & 19176 & 4584 & 28.2 \\
\hline IBM Power 550 (4.2GHz POWER6) & 1 & 13.60 & 26500 & 360 & 16.8 \\
\hline hp server rx5670 (900MHz, 1.5MB L3 Cache) & 4 & 13.53 & 37920 & 1440 & 14.4 \\
\hline IBM Power 520 (4.2GHz POWER6) & 1 & 13.53 & 23700 & 300 & 16.8 \\
\hline
\end{tabular}




\begin{tabular}{|c|c|c|c|c|c|}
\hline $\begin{array}{c}\text { Computer } \\
\text { (Full Precision) }\end{array}$ & $\begin{array}{l}\text { Number } \\
\text { of Procs } \\
\text { or Cores }\end{array}$ & $\begin{array}{c}\boldsymbol{R}_{\max } \\
\text { GFlop/s }\end{array}$ & $\begin{array}{c}N_{\max } \\
\text { Order }\end{array}$ & $\begin{array}{c}N_{1 / 2} \\
\text { Order }\end{array}$ & $\begin{array}{c}\boldsymbol{R}_{\text {Peak }} \\
\text { GFlop/s }\end{array}$ \\
\hline IBM eServer pSeries 655 651(1.3GHz POWER4) & 4 & 13.52 & 36000 & 400 & 20.8 \\
\hline CRAY T3E-1200E (600 MHz) & 16 & 13.41 & 31680 & 2304 & 19.2 \\
\hline Sun HPC 10000(333MHz 4MB L2 Cache) & 24 & 13.39 & 20352 & 1728 & 16.0 \\
\hline Sun Ultra HPC 6000(250 MHz 4MB L2 Cache) & 30 & 13.39 & 19968 & 1920 & 15 \\
\hline Sun HPC 6000(336MHz 4MB L2 Cache) & 22 & 13.33 & 20352 & 1728 & 14.8 \\
\hline IBM eServer BladeCenter JS20 (2.2GHz Power PC) & 2 & 13.27 & 20000 & 2100 & 17.60 \\
\hline SGI Origin $2000(250 \mathrm{MHz})$ & 32 & 13.22 & 30720 & 3200 & 16 \\
\hline Sun HPC 6500(400MHz 8MB L2 Cache) & 18 & 13.05 & 39936 & 1536 & 14.4 \\
\hline IBM eServer pSeries 630 6C4(1.2GHz POWER4+) & 4 & 13.03 & 38000 & 400 & 19.2 \\
\hline IBM eServer pSeries 630 6E4(1.2GHz POWER4+) & 4 & 13.03 & 38000 & 400 & 19.2 \\
\hline HITACHI SR8000-G1/1(450MHz) & 1 & 13.0 & 16000 & 888 & 14.4 \\
\hline HITACHI SR2201/56(150MHz) & 56 & 12.98 & 33600 & 4480 & 17 \\
\hline Cray SV1ex-1-32, 500MHz & 8 & 12.96 & 21672 & 1900 & 16 \\
\hline Sun HPC 6500 Cluster/2 (250 MHz, 4MB L2) & 32 & 12.85 & 17472 & 5376 & 16 \\
\hline Cray T3D $128(150 \mathrm{MHz})$ & 128 & 12.8 & 20736 & 3408 & 19 \\
\hline Sun Ultra HPC 6000(250 MHz 4MB L2 Cache) & 28 & 12.53 & 19968 & 1728 & 14 \\
\hline IBM SP2 thin-node2,SP-sw,256MB/node(66 MHz) & 64 & 12.50 & 39000 & 7000 & 16.8 \\
\hline Intel Paragon XPS-35 (50 MHz) & 296 & 12.5 & 29400 & 5000 & 15 \\
\hline IBM System p5 505 (1.65GHz POWER5) & 2 & 12.47 & 30500 & 1000 & 13.2 \\
\hline Sun Ultra HPC $6000250 \mathrm{MHz}$ (1MB L2 Cache) & 30 & 12.42 & 15700 & 4000 & 15.0 \\
\hline IBM System p5 505 (1.65 GHz POWER5) & 2 & 12.39 & 41200 & 310 & 13.2 \\
\hline DEC $41005 / 400(400 \mathrm{MHz})$ & 32 & 12.37 & 15340 & 6120 & 25.6 \\
\hline Hewlett-Packard N4000 (550 MHz) & 8 & 12.37 & 28000 & 540 & 17.6 \\
\hline Sun HPC 10000(333MHz 4MB L2 Cache) & 22 & 12.32 & 20352 & 1536 & 14.7 \\
\hline Cray SV1-1-32 (300 MHz) & 12 & 12.18 & 25704 & 2700 & 14.7 \\
\hline IBM eServer p5 510 (1.65GHz POWER5) & 2 & 12.14 & 46000 & 300 & 13.2 \\
\hline Sun HPC 6000(336MHz 4MB L2 Cache) & 20 & 12.13 & 20352 & 1536 & 13.4 \\
\hline IBM eServer OpenPower 710 (1.65GHz POWER5) & 2 & 12.12 & 63000 & 1400 & 13.20 \\
\hline HP Integrity rx1620-2 (1.6GHz/3MB Itanium 2) & 2 & 12.05 & 29000 & 360 & 12.8 \\
\hline Sun Ultra HPC 10000(250 MHz 4MB L2 Cache) & 28 & 12.05 & 19968 & 1728 & 14 \\
\hline Fujitsu VPP500/8 (10nsec) & 8 & 12.0 & 14960 & 2216 & 13 \\
\hline HP Integrity rx2620-2 (1.6GHz/3MB Itanium 2) & 2 & 11.98 & 29000 & 360 & 12.8 \\
\hline Sun Fire 6800 (900MHz/8MB L2) & 8 & 11.98 & 28956 & 1800 & 14.4 \\
\hline IBM eServer p5 520 (1650 MHz POWER5) & 2 & 11.78 & 58000 & 320 & 13.2 \\
\hline Sun Ultra HPC 6000(250 MHz 4MB L2 Cache) & 26 & 11.66 & 19968 & 1728 & 13 \\
\hline NEC SX-3/24R (2.5 ns) & 2 & 11.6 & 4352 & 492 & 13 \\
\hline NEC SX-3/42R (2.5 ns) & 4 & 11.6 & 4352 & 516 & 13 \\
\hline Sun HPC 6500(400MHz 8MB L2 Cache) & 16 & 11.60 & 39936 & 1536 & 12.8 \\
\hline HP Integrity Server rx5670 (1500MHz, 6.0MB L3 Cache) & 2 & 11.490 & 35016 & 300 & 12.0 \\
\hline HP Integrity Server rx2600 (1500MHz, 6.0MB L3 Cache) & 2 & 11.420 & 35000 & 300 & 12.0 \\
\hline IBM SP2-T2 (66 MHz) & 64 & 11.4 & 26500 & 6250 & 16 \\
\hline IBM POWER2 Super Chip RS/6000 SP(120 MHz) & 32 & 11.38 & 19500 & 4100 & 15 \\
\hline Sun Ultra HPC 10000(250 MHz 1MB L2 Cache) & 32 & 11.34 & 15000 & 2400 & 16.0 \\
\hline Sun HPC 10000(333MHz 4MB L2 Cache) & 20 & 11.24 & 20352 & 1536 & 13.3 \\
\hline
\end{tabular}




\begin{tabular}{|c|c|c|c|c|c|}
\hline $\begin{array}{c}\text { Computer } \\
\text { (Full Precision) }\end{array}$ & $\begin{array}{l}\text { Number } \\
\text { of Procs } \\
\text { or Cores }\end{array}$ & $\begin{array}{c}\boldsymbol{R}_{\max } \\
\text { GFlop/s }\end{array}$ & $\begin{array}{l}N_{\max } \\
\text { Order }\end{array}$ & $\begin{array}{c}N_{1 / 2} \\
\text { Order }\end{array}$ & $\begin{array}{c}\boldsymbol{R}_{\text {Peak }} \\
\text { GFlop/s }\end{array}$ \\
\hline $\begin{array}{l}\text { Dell PE3250 server (Itanium } 21.5 \mathrm{GHz}, 1.5 \mathrm{MB} \text { L3 } \\
\text { Cache) }\end{array}$ & 2 & 11.23 & 24000 & & 12 \\
\hline Sun Ultra HPC 10000(250 MHz 4MB L2 Cache) & 26 & 11.20 & 19968 & 1728 & 13 \\
\hline Sun HPC 10000(400MHz 4MB L2 Cache) & 16 & 11.11 & 39936 & 1344 & 12.8 \\
\hline IBM SP 2 nodes (222 MHz POWER3) & 16 & 11.08 & 27000 & 3000 & 14.2 \\
\hline Compaq GS140 cluster & 16 & 11.01 & 30712 & 1200 & 17 \\
\hline Sun HPC 6000(336MHz 4MB L2 Cache) & 18 & 10.94 & 20352 & 1344 & 12.1 \\
\hline Cray C90 $(240 \mathrm{MHz})^{* * *}$ & 8 & 10.93 & 13312 & 490 & 7.7 \\
\hline DEC 8400 5/440 (440 MHz) & 24 & 10.9 & 15340 & 4088 & 21.1 \\
\hline SGI Origin 2000 (195 MHz, 4MB L2 Cache) & 32 & 10.9 & 32000 & 6400 & 12.5 \\
\hline HITACHI SR8000-F1/1(375MHz) & 1 & 10.88 & 10728 & 880 & 12 \\
\hline IBM eServer p5 520 Express (1500 MHz POWER5) & 2 & 10.85 & 38000 & 300 & 12.0 \\
\hline Sun Ultra HPC 6000(250 MHz 4MB L2 Cache) & 24 & 10.78 & 19968 & 1728 & 12 \\
\hline HP Exemplar V-Class (240 MHz) & 16 & 10.65 & 14944 & 896 & 15 \\
\hline Hewlett-Packard V2600 (550 MHz) & 8 & 10.59 & 41000 & 880 & 17.6 \\
\hline CRAY T3E-900 (450 MHz) & 16 & 10.45 & 22080 & 2016 & 14 \\
\hline Thinking Machines CM-2 (half precision) & 2048 & 10.4 & 33920 & 14000 & 28 \\
\hline Intel Itanium 1.396 GHz Dual & 2 & 10.36 & 15000 & & 2.8 \\
\hline Sun Ultra HPC 10000(250 MHz 4MB L2 Cache) & 24 & 10.35 & 19968 & 1728 & 12 \\
\hline IBM SP 8 nodes (332 MHz 604e) & 32 & 10.33 & 31600 & 5000 & 21 \\
\hline HP N4000 (440 MHz) & 8 & 10.22 & 28000 & 516 & 14 \\
\hline Intel Delta (40 MHz) & 384 & 10.2 & 20000 & 6000 & 15 \\
\hline $\begin{array}{l}\text { Dell PE3250 server (Itanium } 21.4 \mathrm{GHz}, 1.5 \mathrm{MB} \text { L3 } \\
\text { Cache) }\end{array}$ & 2 & 10.18 & 24000 & & 11.2 \\
\hline Sun HPC 6500(400MHz 8MB L2 Cache) & 14 & 10.16 & 39936 & 1344 & 11.2 \\
\hline Berkeley NOW:UltraSPARC-1(167-Mhz)+Myricom & 100 & 10.14 & 32768 & 8192 & 33.4 \\
\hline Sun HPC 4500(400MHz 4MB L2 Cache) & 14 & 10.05 & 20352 & 1344 & 11.2 \\
\hline IBM SP 8 nodes (200 MHz POWER3) & 16 & 10.04 & 31600 & 2900 & 12.8 \\
\hline NEC SX-3/24 (2.9 ns) & 2 & 10.0 & 4352 & 500 & 11 \\
\hline NEC SX-3/42 (2.9 ns) & 4 & 10.0 & 4608 & 640 & 11 \\
\hline Sun Ultra HPC 6000250 MHz (1MB L2 Cache) & 24 & 9.992 & 15700 & 1632 & 12.0 \\
\hline Sun Ultra HPC 6000(250 MHz 4MB L2 Cache) & 22 & 9.887 & 19968 & 1728 & 11 \\
\hline Sun Fire $6800(750 \mathrm{MHz} / 8 \mathrm{MB}$ L2\$) & 8 & 9.848 & 15180 & & 12 \\
\hline Thinking Machines CM-200 (10 MHz) & 2048 & 9.8 & 29696 & 11264 & 20 \\
\hline Intel Itanium $21.3 \mathrm{GHz}$ & 2 & 9.754 & 24000 & & 10.4 \\
\hline Sun HPC 6000(336MHz 4MB L2 Cache) & 16 & 9.715 & 20352 & 1344 & 10.8 \\
\hline DEC AlphaServer 8400 5/612 (625 MHz) & 16 & 9.592 & 25624 & 3072 & 20 \\
\hline Sun Ultra HPC 10000(250 MHz 4MB L2 Cache) & 22 & 9.513 & 19968 & 1728 & 11 \\
\hline DEC 4100 5/400 (400 MHz) & 24 & 9.48 & 15344 & 3600 & 19.2 \\
\hline Fujitsu VPP5000/1 (3.33nsec) & 1 & 9.475 & 30000 & 340 & 9.6 \\
\hline Cray T94 $(2.2 \mathrm{~ns}) * * *$ & 4 & 9.414 & 8192 & 420 & 7.2 \\
\hline SGI POWER CHALLENGE (90 MHz) & 40 & 9.4 & 27000 & 6775 & 14 \\
\hline Fujitsu VPP300/4E (6.5nsec) & 4 & 9.33 & 28800 & 1280 & 9.6 \\
\hline Fujitsu VPP700/4E (6.5nsec) & 4 & 9.33 & 28800 & 1280 & 9.6 \\
\hline Fujitsu VX/4E (6.5nsec) & 4 & 9.33 & 28800 & 1280 & 9.6 \\
\hline
\end{tabular}




\begin{tabular}{|c|c|c|c|c|c|}
\hline $\begin{array}{c}\text { Computer } \\
\text { (Full Precision) }\end{array}$ & $\begin{array}{l}\text { Number } \\
\text { of Procs } \\
\text { or Cores }\end{array}$ & $\begin{array}{c}\boldsymbol{R}_{\max } \\
\text { GFlop/s }\end{array}$ & $\begin{array}{c}N_{\max } \\
\text { Order }\end{array}$ & $\begin{array}{c}N_{1 / 2} \\
\text { Order }\end{array}$ & $\begin{array}{c}\boldsymbol{R}_{\text {Peak }} \\
\text { GFlop/s }\end{array}$ \\
\hline HP V2500 (8 proc. $440 \mathrm{MHz})$ & 8 & 9.26 & 41000 & 800 & 14.1 \\
\hline HP Exemplar V-Class (200 MHz) & 16 & 9.203 & 14944 & 868 & 12.8 \\
\hline Sun HPC 10000(333MHz 4MB L2 Cache) & 16 & 9.107 & 20352 & 1344 & 10.7 \\
\hline IBM SP 2 nodes (375 MHz POWER3 Thin) & 8 & 9.09 & 27000 & 1700 & 12 \\
\hline HITACHI SR8000-E1/1(300MHz) & 1 & 9.047 & 16000 & 792 & 9.6 \\
\hline HP AlphaServer GS1280 7/1300 (1.3 GHz) & 4 & 9.04 & 26000 & & 10.4 \\
\hline Sun Ultra HPC 6000(250 MHz 4MB L2 Cache) & 20 & 8.997 & 19968 & 1344 & 10 \\
\hline Intel Core 2 Q6600 Kensfield) (1 core, $2.4 \mathrm{GHz}$ ) & 1 & 8.878 & 15000 & 1664 & 9.6 \\
\hline SGI Origin 2000 (300 Mhz) & 16 & 8.712 & 24580 & 1156 & 9.6 \\
\hline Sun HPC 6500(400MHz 8MB L2 Cache) & 12 & 8.711 & 39936 & 1344 & 9.6 \\
\hline NEC SX-3/32R (2.5 ns) & 3 & 8.7 & 6144 & 717 & 9.6 \\
\hline Sun Ultra HPC 10000(250 MHz 4MB L2 Cache) & 20 & 8.679 & 19968 & 1344 & 10 \\
\hline Fujitsu VPP300/4 ( 7nsec) & 4 & 8.6 & 28800 & 1280 & 8.8 \\
\hline Fujitsu VPP700/4 ( 7nsec) & 4 & 8.6 & 28800 & 1280 & 8.8 \\
\hline Fujitsu VX/4 ( 7nsec) & 4 & 8.6 & 28800 & 1280 & 8.8 \\
\hline Sun HPC 6000(336MHz 4MB L2 Cache) & 14 & 8.527 & 20352 & 1344 & 9.4 \\
\hline IBM System p5 575 (2.2GHz POWER5+) & 1 & 8.33 & 20300 & 260 & 8.8 \\
\hline Cray C90 $(240 \mathrm{MHz})^{* * *}$ & 6 & 8.29 & 13312 & 450 & 5.8 \\
\hline SGI POWER CHALLENGE (195 MHz, 2MB cache) & 32 & 8.233 & 16000 & 4000 & 12 \\
\hline HP Exemplar V-Class (240 MHz) & 12 & 8.228 & 14944 & 736 & 11.5 \\
\hline Cray SV1-1-32 (300 MHz) & 8 & 8.150 & 21672 & 1900 & 9.6 \\
\hline Sun Ultra HPC 6000(250 MHz 4MB L2 Cache) & 18 & 8.113 & 19968 & 1344 & 9 \\
\hline Parsytec GC/Power Plus (80 MHz) & 192 & 8.0 & 27192 & 9500 & 15 \\
\hline HP AlphaServer ES80 7/1150 (1.15 GHz) & 4 & 7.93 & 26000 & & 9.2 \\
\hline HP AlphaServer ES47 7/1150 (1.15 GHz) & 4 & 7.93 & 26000 & & 9.2 \\
\hline SGI Origin 2000 (195 MHz, 4MB cache) & 24 & 7.928 & 19000 & 3500 & 9.4 \\
\hline hp AlphaServer GS1280 7/1150(1.15 GHz) & 4 & 7.82 & 20000 & & 9.2 \\
\hline Sun Ultra HPC 6000167 MHz (1MB L2 Cache) & 30 & 7.806 & 14000 & 1000 & 10.0 \\
\hline Paderborn SCI Cluster:SNI/Scali(300MHz PII) & 64 & 7.8 & 28000 & 8000 & 19.2 \\
\hline SGI POWER CHALLENGE (75 MHz) & 40 & 7.8 & 27000 & 6775 & 12 \\
\hline HP Exemplar S-Class SPP-UX 5.2 & 16 & 7.783 & 13320 & 1044 & 11.5 \\
\hline DEC 8400 5/440 (440 MHz) & 16 & 7.7 & 15340 & 3270 & 14.1 \\
\hline Thinking Machines CM-5 & 128 & 7.7 & 18432 & 8192 & 16 \\
\hline IBM IntelliStation POWER 275 (1.45GHz POWER4+) & 2 & 7.69 & 26000 & 300 & 11.6 \\
\hline SGI POWER CHALLENGE (195 MHz, 2MB cache) & 28 & 7.635 & 15000 & 4000 & 11 \\
\hline Cray J932 (10 ns) *** & 32 & 7.622 & 19456 & 800 & 6.4 \\
\hline Intel Paragon XPS-35 (50 MHz, OS=R1.1) & 256 & 7.6 & 16000 & 4000 & 13 \\
\hline IBM SP2 (160 MHz) & 16 & 7.57 & 13500 & 2280 & 10 \\
\hline HITACHI SR8000/1(250MHz) & 1 & 7.50 & 10728 & 696 & 8 \\
\hline SGI POWER CHALLENGE (90 MHz) & 32 & 7.5 & 22000 & 5600 & 16 \\
\hline Cray J928 (10 ns) *** & 28 & 7.413 & 19456 & 750 & 5.6 \\
\hline Hitachi S-3800/180 (2 ns) & 1 & 7.4 & 15680 & 470 & 8 \\
\hline IBM SP2 (77 MHz, switch of 4/96) & 32 & 7.3 & 19500 & 3500 & 10 \\
\hline DEC 8400 5/625 (612 MHz) & 12 & 7.283 & 9548 & 1800 & 14.7 \\
\hline
\end{tabular}




\begin{tabular}{|c|c|c|c|c|c|}
\hline $\begin{array}{c}\text { Computer } \\
\text { (Full Precision) }\end{array}$ & $\begin{array}{l}\text { Number } \\
\text { of Procs } \\
\text { or Cores }\end{array}$ & $\begin{array}{c}\boldsymbol{R}_{\max } \\
\text { GFlop/s }\end{array}$ & $\begin{array}{c}N_{\max } \\
\text { Order }\end{array}$ & $\begin{array}{c}N_{1 / 2} \\
\text { Order }\end{array}$ & $\begin{array}{c}\boldsymbol{R}_{\text {Peak }} \\
\text { GFlop/s }\end{array}$ \\
\hline IBM System p5 505 (1.9 GHz POWER5+) & 1 & 7.281 & 29100 & 200 & 7.6 \\
\hline IBM eServer pSeries 650 6M2(1.45GHz POWER4+) & 2 & 7.28 & 24000 & 300 & 11.6 \\
\hline Sun HPC 6500(400MHz 8MB L2 Cache) & 10 & 7.266 & 39936 & 1344 & 8.0 \\
\hline IBM System p5 550 (1.9GHz POWER5+) & 1 & 7.254 & 26550 & 230 & 7.6 \\
\hline Sun Ultra HPC 6000(250 MHz 4MB L2 Cache) & 16 & 7.219 & 19968 & 1344 & 8 \\
\hline Cray T3E-1350 (675 MHz) & 8 & 7.2 & 22272 & 1536 & 10.8 \\
\hline IBM System p5 575 (1.9GHz POWER5+) & 1 & 7.14 & 23100 & 820 & 7.6 \\
\hline CRAY T3E (300 MHz) & 16 & 7.133 & 14976 & 1728 & 9.6 \\
\hline IBM eServer p5 575 (1.9GHz POWER5) & 1 & 7.12 & 40000 & 230 & 7.6 \\
\hline Cray T94 $(2.2 \mathrm{~ns}) * * *$ & 3 & 7.112 & 8192 & 370 & 5.4 \\
\hline HP Exemplar V-Class (200 MHz) & 12 & 7.094 & 14944 & 696 & 9.6 \\
\hline Sun Ultra HPC 10000(250 MHz 4MB L2 Cache) & 16 & 7.023 & 19968 & 1344 & 8 \\
\hline Intel Delta $(40 \mathrm{MHz})$ & 256 & 7.0 & 18000 & 5000 & 10 \\
\hline DEC $41005 / 400(400 \mathrm{MHz})$ & 16 & 6.89 & 15344 & 2760 & 12.8 \\
\hline SGI POWER CHALLENGE (195 MHz, 2MB cache) & 24 & 6.819 & 15000 & 3500 & 9.4 \\
\hline IBM System p5 560Q (1.5GHz POWER5) & 1 & 6.8 & 23100 & 200 & 7.2 \\
\hline Sun Ultra HPC $6000250 \mathrm{MHz}$ (1MB L2 Cache) & 16 & 6.688 & 15700 & 1088 & 8.0 \\
\hline DEC Alphaserver 8400 5/440(440MHz, 4MB cache) & 12 & 6.678 & 9548 & 1028 & 10.6 \\
\hline CRAY T3E-1200E (600 MHz) & 8 & 6.674 & 22272 & 1536 & 9.6 \\
\hline Cray J924 (10 ns) *** & 24 & 6.645 & 19456 & 700 & 4.8 \\
\hline IBM SP2 thin-node2,SP-sw,256MB/node( $66 \mathrm{MHz})$ & 32 & 6.569 & 28000 & 5200 & 8.4 \\
\hline Hewlett-Packard N4000 (550 MHz) & 4 & 6.568 & 28000 & 376 & 8.8 \\
\hline Cray SV1ex-1-32, 500MHz & 4 & 6.527 & 15372 & 1250 & 8 \\
\hline Compaq Alphaserver ES45 (1001Mhz 8MB L2) & 4 & 6.435 & 14000 & 1050 & 8.0 \\
\hline Cray T3D $64(150 \mathrm{MHz})$ & 64 & 6.4 & 20736 & 2368 & 9.6 \\
\hline Sun Ultra HPC 6000167 MHz (1MB L2 Cache) & 24 & 6.350 & 14000 & 800 & 8.0 \\
\hline Sun Ultra HPC $6000250 \mathrm{MHz}$ (4MB L2 Cache) & 14 & 6.251 & 15552 & 1152 & 7.0 \\
\hline IBM System p5 505 (1.65 GHz POWER5) & 1 & 6.231 & 29100 & 200 & 6.6 \\
\hline Convex SPP-1000(64 procs) $100 \mathrm{MHz}$ & 64 & 6.192 & 41000 & 11400 & 12.8 \\
\hline SGI POWER CHALLENGE (195 MHz, 1 MB cache) & 24 & 6.118 & 15000 & 3100 & 9.3 \\
\hline Fujitsu VPP500/4 (10nsec) & 4 & 6.1 & 10560 & 1390 & 6.4 \\
\hline Sun Fire $6800(900 \mathrm{MHz} / 8 \mathrm{MB} \mathrm{L} 2)$ & 4 & 6.016 & 28956 & 1200 & 7.2 \\
\hline HP Exemplar S-Class SPP-UX 5.2 & 12 & 6.005 & 13320 & 800 & 8.6 \\
\hline Cray J920 (10 ns) *** & 20 & 5.917 & 19456 & 675 & 4.0 \\
\hline DEC $84005 / 350(12 \operatorname{proc} 350 \mathrm{MHz})$ & 12 & 5.904 & 9548 & 3010 & 8.4 \\
\hline SGI POWER CHALLENGE (195 MHz, 2MB cache) & 20 & 5.872 & 15000 & 3000 & 7.8 \\
\hline Sun Ultra HPC $6000250 \mathrm{MHz}$ (1MB L2 Cache) & 14 & 5.856 & 15700 & 960 & 7.0 \\
\hline DEC Alphaserver $84005 / 440(440 \mathrm{MHz}, 4 \mathrm{MB}$ cache $)$ & 10 & 5.845 & 9548 & 1124 & 8.8 \\
\hline SGI POWER CHALLENGE (195 MHz, 1 MB cache) & 22 & 5.812 & 15000 & 2900 & 8.6 \\
\hline Sun HPC 6500(400MHz 8MB L2 Cache) & 8 & 5.810 & 39936 & 1344 & 6.4 \\
\hline IBM SP2-T2 (66 MHz) & 32 & 5.8 & 18000 & 4500 & 8.4 \\
\hline NEC SX-3/14R (2.5 ns) & 1 & 5.8 & 2816 & 282 & 6.4 \\
\hline NEC SX-3/22R (2.5 ns) & 2 & 5.8 & 3072 & 370 & 6.4 \\
\hline NEC SX-3/41R (2.5 ns) & 4 & 5.8 & 3584 & 414 & 6.4 \\
\hline Sun HPC 4500(400MHz 4MB L2 Cache) & 8 & 5.772 & 20352 & 960 & 6.4 \\
\hline
\end{tabular}




\begin{tabular}{|c|c|c|c|c|c|}
\hline $\begin{array}{c}\text { Computer } \\
\text { (Full Precision) }\end{array}$ & $\begin{array}{l}\text { Number } \\
\text { of Procs } \\
\text { or Cores }\end{array}$ & $\begin{array}{c}\boldsymbol{R}_{\max } \\
\text { GFlop/s }\end{array}$ & $\begin{array}{l}N_{\max } \\
\text { Order }\end{array}$ & $\begin{array}{c}N_{1 / 2} \\
\text { Order }\end{array}$ & $\begin{array}{c}\boldsymbol{R}_{\text {Peak }} \\
\text { GFlop/s }\end{array}$ \\
\hline IBM POWER2 Super Chip RS/6000 SP(120 MHz) & 16 & 5.767 & 13500 & 2600 & 7.7 \\
\hline Cray C90 $(240 \mathrm{MHz})^{* * *}$ & 4 & 5.75 & 13312 & 420 & 3.8 \\
\hline HP Integrity Server rx2600 (1500MHz, 6.0MB L3 Cache) & 1 & 5.711 & 30000 & 300 & 6.0 \\
\hline HP Integrity Server rx5670 (1500MHz, 6.0MB L3 Cache) & 1 & 5.683 & 35016 & 300 & 6.0 \\
\hline HP Exemplar V-Class (240 MHz) & 8 & 5.657 & 14944 & 560 & 7.68 \\
\hline Hewlett-Packard V2600 (550 MHz) & 4 & 5.650 & 41000 & 600 & 8.8 \\
\hline IBM System p5 560Q (1.8GHz POWER5+) & 1 & 5.65 & 23000 & 240 & 6.0 \\
\hline IBM GF11** (half precision) (51.9 ns) & 500 & 5.6 & 2500 & 1060 & 9.6 \\
\hline IBM System p5 550Q (1.5GHz POWER5+) & 1 & 5.596 & 20500 & 220 & 6.0 \\
\hline IBM SP 1 node (222 MHz POWER3) & 8 & 5.54 & 13000 & 800 & 7.1 \\
\hline Convex SPP-1600(32 procs) $120 \mathrm{MHz}$ & 32 & 5.452 & 27000 & 4500 & 7.7 \\
\hline SGI POWER CHALLENGE (195 MHz, 1 MB cache) & 20 & 5.430 & 15000 & 2600 & 7.8 \\
\hline HP N4000 (440 MHz) & 4 & 5.394 & 28000 & 356 & 7.0 \\
\hline IBM SP 4 nodes (332 MHz 604e) & 16 & 5.37 & 22400 & 3200 & 11 \\
\hline SGI Origin 2000 (195 MHz, 4MB cache) & 16 & 5.300 & 16000 & 1000 & 6.2 \\
\hline CRAY T3E-900 (450 MHz) & 8 & 5.243 & 15552 & 1488 & 7.2 \\
\hline Intel Delta (40 MHz) & 192 & 5.2 & 15000 & 4500 & 7.7 \\
\hline Parsytec GC/Power Plus (80 MHz) & 128 & 5.2 & 22000 & 7800 & 10 \\
\hline Thinking Machines CM-2 (7 MHz) & 2048 & 5.2 & 26624 & 11000 & 14 \\
\hline IBM SP 4 nodes (200 MHz POWER3) & 8 & 5.13 & 22400 & 1600 & 6.4 \\
\hline Compaq ES40/EV67 AlphaServer SC (833 MHz) & 4 & 5.105 & 12800 & 1000 & 6.66 \\
\hline DEC AlphaServer 8400 5/300 & 12 & 5.0 & 9548 & 1148 & 7.2 \\
\hline Meiko CS2 & 64 & 5.0 & 18688 & 6144 & 11.5 \\
\hline NEC SX-3/14 (2.9 ns) & 1 & 5.0 & 3072 & 384 & 5.5 \\
\hline NEC SX-3/22 (2.9 ns) & 2 & 5.0 & 3072 & 384 & 5.5 \\
\hline Thinking Machines CM-200 (10 MHz) & 1024 & 5.0 & 21504 & 8192 & 10 \\
\hline SGI POWER CHALLENGE (195 MHz, 2MB cache) & 18 & 4.992 & 15000 & 2350 & 7.0 \\
\hline Sun Fire $6800(750 \mathrm{MHz} / 8 \mathrm{MB}$ L2\$) & 4 & 4.968 & 15180 & & 6 \\
\hline Intel Pentium 4 3.0 GHz (Northwood core) & 1 & 4.937 & 12800 & & 6 \\
\hline Cray J916 (10 ns) *** & 16 & 4.911 & 19456 & 640 & 3.2 \\
\hline SGI POWER CHALLENGE (75 MHz) & 24 & 4.9 & 18000 & 3500 & 7.2 \\
\hline Cray T94 $(2.2 \mathrm{~ns}) * * *$ & 2 & 4.886 & 8192 & 350 & 3.6 \\
\hline Sun HPC 6000(336MHz 4MB L2 Cache) & 8 & 4.886 & 20352 & 960 & 5.4 \\
\hline IBM eServer pSeries 655 (1.7GHz POWER4+) & 1 & 4.87 & 38000 & 200 & 6.8 \\
\hline SGI POWER CHALLENGE (195 MHz, 2MB cache) & 16 & 4.862 & 15000 & 2500 & 6.2 \\
\hline HP Exemplar V-Class (200 MHz) & 8 & 4.860 & 14944 & 552 & 6.4 \\
\hline Alliant CAMPUS/800 (40 MHz) & 192 & 4.8 & 17024 & 5768 & 7.7 \\
\hline IBM SP-1 & 64 & 4.8 & 26000 & 6000 & 8 \\
\hline DEC Alphaserver 8400 5/440(440MHz, 4MB cache) & 8 & 4.754 & 7644 & 1500 & 7.0 \\
\hline HP V2500 (4 proc. $440 \mathrm{MHz})$ & 4 & 4.70 & 41000 & 600 & 7.04 \\
\hline IBM eServer pSeries 640 (375 MHz, 8MB L2) & 4 & 4.64 & 19000 & 340 & 6 \\
\hline IBM RS/6000 44P-270 (375 MHz, 8MB L2) & 4 & 4.64 & 19000 & 340 & 6 \\
\hline IBM RS/6000 44P-270 (4 proc,375 MHz,8 MB L2) & 4 & 4.64 & 19000 & 340 & 6 \\
\hline IBM SP 1 node (375 MHz POWER3 Thin) & 4 & 4.62 & 19000 & 440 & 6 \\
\hline
\end{tabular}




\begin{tabular}{|c|c|c|c|c|c|}
\hline $\begin{array}{c}\text { Computer } \\
\text { (Full Precision) }\end{array}$ & $\begin{array}{l}\text { Number } \\
\text { of Procs } \\
\text { or Cores }\end{array}$ & $\begin{array}{c}\boldsymbol{R}_{\max } \\
\text { GFlop/s }\end{array}$ & $\begin{array}{l}N_{\max } \\
\text { Order }\end{array}$ & $\begin{array}{c}N_{1 / 2} \\
\text { Order }\end{array}$ & $\begin{array}{c}\boldsymbol{R}_{\text {Peak }} \\
\text { GFlop/s }\end{array}$ \\
\hline SGI POWER CHALLENGE (90 MHz) & 18 & 4.620 & 2500 & 540 & 6.5 \\
\hline Intel Pentium 4 3.0 GHz (Northwood core) & 1 & 4.725 & 7600 & 365 & 6 \\
\hline Compaq Digital AlphaServer 8400 (575 MHz) & 6 & 4.600 & 11504 & 900 & 6.9 \\
\hline IBM eServer pSeries 640 (375 MHz, 4MB L2) & 4 & 4.53 & 19000 & 400 & 6 \\
\hline IBM RS/6000 44P-270 (375 MHz, 4MB L2) & 4 & 4.53 & 19000 & 400 & 6 \\
\hline IBM RS/6000 44P-270 (4 proc,375 MHz,4 MB L2) & 4 & 4.53 & 19000 & 180 & 6 \\
\hline IBM RS/6000 7026-B80(4 proc,375 MHz,4 MB L2) & 4 & 4.53 & 19000 & 400 & 6 \\
\hline SGI POWER CHALLENGE (195 MHz, 1 MB cache) & 16 & 4.527 & 15000 & 2200 & 6.2 \\
\hline HP AlphaServer GS1280 7/1300 (1.3 GHz) & 2 & 4.52 & 14142 & & 5.2 \\
\hline Compaq Digital AlphaServer 8200 (575 MHz) & 6 & 4.450 & 11504 & 800 & 6.9 \\
\hline NEC SX-3/31R (2.5 ns) & 3 & 4.4 & 6144 & 414 & 5.4 \\
\hline Sun HPC 6500(400MHz 8MB L2 Cache) & 6 & 4.356 & 39936 & 768 & 4.8 \\
\hline Sun HPC 4500(400MHz 4MB L2 Cache) & 6 & 4.334 & 20352 & 960 & 4.8 \\
\hline SGI POWER CHALLENGE (90 MHz) & 16 & 4.323 & 2500 & 540 & 5.8 \\
\hline Cray C90 $(240 \mathrm{MHz})^{* * *}$ & 3 & 4.31 & 13312 & 380 & 2.9 \\
\hline Sun Ultra HPC 6000167 MHz (1MB L2 Cache) & 16 & 4.305 & 14000 & 700 & 5.3 \\
\hline SGI POWER CHALLENGE (75 MHz) & 18 & 4.142 & 2604 & 570 & 5.4 \\
\hline Compaq ES40/EV67 AlphaServer SC (667 MHz) & 4 & 4.111 & 10000 & 850 & 5.34 \\
\hline Cray SV1-1-32 (300 MHz) & 4 & 4.105 & 15372 & 1250 & 4.8 \\
\hline HP Exemplar S-Class SPP-UX 5.2 & 8 & 4.103 & 13320 & 520 & 5.8 \\
\hline Alliant CAMPUS/800 (40 MHz) & 168 & 4.1 & 16016 & 5516 & 6.7 \\
\hline SGI POWER CHALLENGE (195 MHz, 1 MB cache) & 14 & 4.041 & 15000 & 2000 & 5.5 \\
\hline SGI Origin 2000 (195 MHz, 4MB cache) & 12 & 4.038 & 15000 & 1000 & 4.7 \\
\hline IBM IntelliStation POWER 275 (1.45GHz POWER4+) & 1 & 4.02 & 26000 & 200 & 5.8 \\
\hline DEC 8400 5/625 (612 MHz) & 6 & 4.003 & 9156 & 1100 & 7.34 \\
\hline Intel Paragon XPS-35 (50 MHz, OS=R1.1) & 128 & 4.0 & 12000 & 3000 & 6.4 \\
\hline hp AlphaServer GS1280 7/1150(1.15 GHz) & 2 & 3.98 & 7500 & & 4.6 \\
\hline HP AlphaServer ES80 7/1150 (1.15 GHz) & 2 & 3.97 & 14142 & & 4.6 \\
\hline HP AlphaServer ES47 7/1150 (1.15 GHz) & 2 & 3.97 & 14142 & & 4.6 \\
\hline Convex SPP-1200(32 procs) $120 \mathrm{MHz}$ & 32 & 3.962 & 27700 & 4500 & 7.7 \\
\hline DEC AlphaServer 8400 5/300 & 10 & 3.9 & 9540 & 812 & 6.0 \\
\hline Parsytec GC/Power Plus (80 MHz) & 96 & 3.9 & 19000 & 6599 & 7.7 \\
\hline IBM SP2 (160 MHz) & 8 & 3.83 & 10000 & 1320 & 5.1 \\
\hline Thinking Machines CM-5 & 64 & 3.8 & 13056 & 6016 & 8 \\
\hline Cray J912 (10 ns) *** & 12 & 3.768 & 19456 & 690 & 2.4 \\
\hline SGI POWER CHALLENGE (90 MHz) & 14 & 3.767 & 2000 & 470 & 5.0 \\
\hline HITACHI SR2201/16(150MHz) & 16 & 3.74 & 19440 & 2880 & 4.8 \\
\hline IBM SP2 (77 MHz, switch of 4/96) & 16 & 3.7 & 13500 & 2200 & 5 \\
\hline SGI POWER CHALLENGE (75 MHz) & 16 & 3.7 & 2500 & 540 & 4.8 \\
\hline IBM eServer pSeries 650 6M2(1.45GHz POWER4+) & 1 & 3.68 & 24000 & 200 & 5.8 \\
\hline Sun HPC 6000(336MHz 4MB L2 Cache) & 6 & 3.672 & 20352 & 960 & 4.0 \\
\hline SGI POWER CHALLENGE (195 MHz, 2MB cache) & 12 & 3.604 & 10000 & 2000 & 4.7 \\
\hline Sun Ultra HPC $6000250 \mathrm{MHz}$ (4MB L2 Cache) & 8 & 3.589 & 15552 & 768 & 4.0 \\
\hline DEC 4100 5/400 (400 MHz) & 8 & 3.57 & 8964 & 1340 & 6.4 \\
\hline
\end{tabular}




\begin{tabular}{|c|c|c|c|c|c|}
\hline $\begin{array}{c}\text { Computer } \\
\text { (Full Precision) }\end{array}$ & $\begin{array}{l}\text { Number } \\
\text { of Procs } \\
\text { or Cores }\end{array}$ & $\begin{array}{c}\boldsymbol{R}_{\max } \\
\text { GFlop/s }\end{array}$ & $\begin{array}{c}N_{\max } \\
\text { Order }\end{array}$ & $\begin{array}{c}N_{1 / 2} \\
\text { Order }\end{array}$ & $\begin{array}{c}\boldsymbol{R}_{\text {Peak }} \\
\text { GFlop/s }\end{array}$ \\
\hline CRAY T3E (300 MHz) & 8 & 3.542 & 10560 & 1152 & 4.8 \\
\hline Alliant CAMPUS/800 (40 MHz) & 144 & 3.5 & 15484 & 4956 & 5.8 \\
\hline Intel Delta (40 MHz) & 128 & 3.5 & 12500 & 3500 & 5 \\
\hline SGI POWER CHALLENGE (195 MHz, 1 MB cache) & 12 & 3.496 & 15000 & 1650 & 4.7 \\
\hline IBM eServer pSeries 655 651(1.3GHz POWER4) & 1 & 3.45 & 24000 & 200 & 5.2 \\
\hline IBM SP2 thin-node2,SP-sw,256MB/node(66 MHz) & 16 & 3.414 & 19000 & 3400 & 4.2 \\
\hline SGI POWER CHALLENGE (90 MHz) & 12 & 3.398 & 2000 & 450 & 4.3 \\
\hline Hewlett-Packard N4000 (550 MHz) & 2 & 3.391 & 28000 & 276 & 4.4 \\
\hline CRAY T3E-1200E (600 MHz) & 4 & 3.372 & 15936 & 960 & 4.8 \\
\hline Sun Ultra HPC 6000250 MHz (1MB L2 Cache) & 8 & 3.328 & 15700 & 700 & 4.0 \\
\hline Cray SV1ex-1-32, 500MHz & 2 & 3.318 & 11088 & 600 & 4.0 \\
\hline Convex SPP-1000(32 procs) $100 \mathrm{MHz}$ & 32 & 3.306 & 25800 & 4700 & 6.4 \\
\hline Intel Pentium $4(2.53 \mathrm{GHz})$ & 1 & 3.210 & 9000 & 340 & 5.09 \\
\hline SGI POWER CHALLENGE (75 MHz) & 14 & 3.203 & 2000 & 470 & 4.2 \\
\hline Cray T3D $32(150 \mathrm{MHz})$ & 32 & 3.2 & 14592 & 1616 & 3.6 \\
\hline DEC AlphaServer 8400 5/300 & 8 & 3.2 & 7668 & 540 & 4.8 \\
\hline Sun Ultra 80 (450MHz/4MB L2) & 4 & 3.090 & 20352 & 576 & 3.6 \\
\hline IBM SP2-T2 (66 MHz) & 16 & 3.0 & 13000 & 2600 & 4.2 \\
\hline IBM eServer pSeries 655 651(1.1GHz POWER4) & 1 & 2.93 & 24000 & 200 & 4.4 \\
\hline Cray C90 $(240 \mathrm{MHz})^{* * *}$ & 2 & 2.92 & 13312 & 350 & 1.9 \\
\hline HP Exemplar V-Class (240 MHz) & 4 & 2.910 & 14944 & 400 & 3.84 \\
\hline Alliant CAMPUS/800 (40 MHz) & 120 & 2.9 & 14000 & 4620 & 4.8 \\
\hline NEC SX-3/12R (2.5 ns) & 1 & 2.9 & 2048 & 174 & 3.2 \\
\hline NEC SX-3/21R (2.5 ns) & 2 & 2.9 & 2560 & 257 & 3.2 \\
\hline Sun HPC 6500(400MHz 8MB L2 Cache) & 4 & 2.898 & 39936 & 576 & 3.2 \\
\hline Sun HPC 4500(400MHz 4MB L2 Cache) & 4 & 2.893 & 20352 & 960 & 3.2 \\
\hline Sun HPC $450(400 \mathrm{MHz})$ & 4 & 2.879 & 20252 & 960 & 3.2 \\
\hline IBM POWER2 Super Chip RS/6000 SP(120 MHz) & 8 & 2.876 & 9500 & 1500 & 3.8 \\
\hline SGI POWER CHALLENGE (75 MHz) & 12 & 2.874 & 2000 & 450 & 3.6 \\
\hline Convex SPP-1600(16 procs) $120 \mathrm{MHz}$ & 16 & 2.840 & 18000 & 2400 & 3.8 \\
\hline Convex SPP-1200(24 procs) $120 \mathrm{MHz}$ & 24 & 2.830 & 21100 & 3400 & 5.8 \\
\hline SGI POWER CHALLENGE (90 MHz) & 10 & 2.830 & 2000 & 400 & 3.6 \\
\hline IBM IntelliStation POWER 275 (1GHz POWER4+) & 1 & 2.82 & 26000 & 200 & 4.0 \\
\hline Meiko CS2 & 32 & 2.8 & 13824 & 3488 & 5.8 \\
\hline Parsytec GC/Power Plus (80 MHz) & 64 & 2.8 & 16000 & 4500 & 5.1 \\
\hline HP N4000 (440 MHz) & 2 & 2.761 & 28000 & 268 & 3.5 \\
\hline Sun Ultra HPC $6000250 \mathrm{MHz}$ (4MB L2 Cache) & 6 & 2.694 & 15552 & 672 & 3.0 \\
\hline SGI Origin 2000 (195 MHz, 4MB cache) & 8 & 2.678 & 10000 & 1000 & 3.1 \\
\hline CRAY T3E-900 (450 MHz) & 4 & 2.630 & 11040 & 880 & 3.6 \\
\hline Intel iPSC/860 (40 MHz) & 128 & 2.6 & 12000 & 4500 & 5. \\
\hline Cray J908 (10 ns) *** & 8 & 2.585 & 19456 & 520 & 1.6 \\
\hline SGI POWER CHALLENGE (195 MHz, 2MB cache) & 8 & 2.513 & 10000 & 1500 & 3.1 \\
\hline NEC SX-3/12 (2.9 ns) & 1 & 2.5 & 2048 & 256 & 2.8 \\
\hline HP Exemplar V-Class (200 MHz) & 4 & 2.495 & 14944 & 384 & 3.2 \\
\hline
\end{tabular}




\begin{tabular}{|c|c|c|c|c|c|}
\hline $\begin{array}{c}\text { Computer } \\
\text { (Full Precision) }\end{array}$ & $\begin{array}{l}\text { Number } \\
\text { of Procs } \\
\text { or Cores }\end{array}$ & $\begin{array}{c}\boldsymbol{R}_{\max } \\
\text { GFlop/s }\end{array}$ & $\begin{array}{c}N_{\max } \\
\text { Order }\end{array}$ & $\begin{array}{c}N_{1 / 2} \\
\text { Order }\end{array}$ & $\begin{array}{c}\boldsymbol{R}_{\text {Peak }} \\
\text { GFlop/s }\end{array}$ \\
\hline Sun Fire $6800(750 \mathrm{MHz} / 8 \mathrm{MB}$ L2\$) & 2 & 2.486 & 15180 & & 3 \\
\hline Sun Ultra HPC $6000250 \mathrm{MHz}$ (1MB L2 Cache) & 6 & 2.483 & 15700 & 700 & 3.0 \\
\hline Cray T94 $(2.2 \mathrm{~ns}) * * *$ & 1 & 2.474 & 8192 & 280 & 1.8 \\
\hline Sun HPC 6000(336MHz 4MB L2 Cache) & 4 & 2.452 & 20352 & 960 & 2.7 \\
\hline DEC AlphaServer $82005 / 300$ & 6 & 2.4 & 9640 & 540 & 3.6 \\
\hline DEC AlphaServer $84005 / 300$ & 6 & 2.4 & 9640 & 540 & 3.6 \\
\hline IBM SP-1 & 32 & 2.4 & 16000 & 4000 & 4 \\
\hline Thinking Machines CM-200 (10 MHz) & 512 & 2.4 & 14848 & 5632 & 5 \\
\hline SGI POWER CHALLENGE (75 MHz) & 10 & 2.395 & 2000 & 470 & 3.0 \\
\hline IBM eServer pSeries 640 (375 MHz, 8MB L2) & 2 & 2.38 & 12000 & 200 & 3 \\
\hline IBM RS/6000 44P-270 (2 proc,375 MHz,8 MB L2) & 2 & 2.38 & 12000 & 200 & 3 \\
\hline IBM RS/6000 44P-270 (375 MHz, 8MB L2) & 2 & 2.38 & 12000 & 200 & 3 \\
\hline SGI POWER CHALLENGE (90 MHz) & 8 & 2.318 & 1900 & 360 & 2.9 \\
\hline Alliant CAMPUS/800 (40 MHz) & 96 & 2.3 & 13020 & 4396 & 3.8 \\
\hline Fujitsu AP1000 & 512 & 2.3 & 25600 & 2500 & 2.8 \\
\hline Intel iPSC/860 (40 MHz) & 120 & 2.3 & 12000 & 4500 & 4.8 \\
\hline HP AlphaServer GS1280 7/1300 (1.3 GHz) & 1 & 2.27 & 10000 & & 2.6 \\
\hline IBM eServer pSeries 640 (375 MHz, 4MB L2) & 2 & 2.27 & 13000 & 180 & 3 \\
\hline IBM RS/6000 44P-270 (2 proc,375 MHz,4 MB L2) & 2 & 2.27 & 13000 & 400 & 3 \\
\hline IBM RS/6000 44P-270 (375 MHz, 4MB L2) & 2 & 2.27 & 13000 & 180 & 3 \\
\hline IBM RS/6000 7026-B80(2 proc,375 MHz,4 MB L2) & 2 & 2.27 & 13000 & 180 & 3 \\
\hline Sun Ultra HPC 6000167 MHz (1MB L2 Cache) & 8 & 2.185 & 14000 & 500 & 2.7 \\
\hline HP Exemplar S-Class SPP-UX 5.2 & 4 & 2.121 & 13320 & 520 & 2.9 \\
\hline Sun Ultra HPC $450(300 \mathrm{MHz})$ & 4 & 2.09 & 10944 & 492 & 2.4 \\
\hline Cray SV1-1-32 (300 MHz) & 2 & 2.073 & 11088 & 600 & 2.4 \\
\hline Convex SPP-1200(16 procs) $120 \mathrm{MHz}$ & 16 & 2.032 & 19000 & 2800 & 3.8 \\
\hline DEC 4100 5/400 (400 MHz) & 4 & 2.019 & 4929 & 1280 & 3.2 \\
\hline HP AlphaServer ES80 7/1150 (1.15 GHz) & 1 & 2.01 & 10000 & & 2.3 \\
\hline HP AlphaServer ES47 7/1150 (1.15 GHz) & 1 & 2.01 & 10000 & & 2.3 \\
\hline hp AlphaServer GS1280 7/1150(1.15 GHz) & 1 & 2.00 & 5000 & & 2.3 \\
\hline Intel Paragon XPS-35 (50 MHz, OS=R1.1) & 64 & 2.0 & 8000 & 2000 & 3.2 \\
\hline SGI POWER CHALLENGE (75 MHz) & 8 & 1.955 & 1900 & 360 & 2.4 \\
\hline Intel iPSC/860 (40 MHz) & 96 & 1.9 & 11000 & 4000 & 3.8 \\
\hline nCUBE $2(20 \mathrm{MHz})$ & 1024 & 1.9 & 21376 & 3193 & 2.4 \\
\hline Thinking Machines CM-5 & 32 & 1.9 & 9216 & 4096 & 4 \\
\hline CRAY T3E (300 MHz) & 4 & 1.806 & 7488 & 768 & 2.4 \\
\hline IBM SP2 (77 MHz, switch of 4/96) & 8 & 1.8 & 9500 & 1200 & 2.5 \\
\hline Sun Ultra HPC 6000250 MHz (4MB L2 Cache) & 4 & 1.798 & 15552 & 576 & 2.0 \\
\hline IBM SP2 thin-node2,SP-sw,256MB/node(66 MHz) & 8 & 1.768 & 12000 & 1700 & 2.1 \\
\hline AMD ATHLON Thunderbird $1.2 \mathrm{GHz}$ & 1 & 1.755 & 3800 & 295 & 2.4 \\
\hline Intel Delta (40 MHz) & 64 & 1.7 & 8000 & 2500 & 2.6 \\
\hline SGI POWER CHALLENGE (90 MHz) & 6 & 1.690 & 2000 & 294 & 2.2 \\
\hline CRAY T3E-1200E (600 MHz) & 2 & 1.675 & 11040 & 576 & 2.4 \\
\hline Cray SV1ex-1-32, 500MHz & 1 & 1.671 & 7452 & 350 & 2.0 \\
\hline
\end{tabular}




\begin{tabular}{|c|c|c|c|c|c|}
\hline $\begin{array}{c}\text { Computer } \\
\text { (Full Precision) }\end{array}$ & $\begin{array}{l}\text { Number } \\
\text { of Procs } \\
\text { or Cores }\end{array}$ & $\begin{array}{c}\boldsymbol{R}_{\max } \\
\text { GFlop/s }\end{array}$ & $\begin{array}{c}N_{\max } \\
\text { Order }\end{array}$ & $\begin{array}{c}N_{1 / 2} \\
\text { Order }\end{array}$ & $\begin{array}{c}\boldsymbol{R}_{\text {Peak }} \\
\text { GFlop/s }\end{array}$ \\
\hline Alliant CAMPUS/800 (40 MHz) & 72 & 1.6 & 12012 & 3724 & 2.9 \\
\hline MasPar MP-2216 (80ns) & 16384 & 1.6 & 11264 & 1920 & 2.4 \\
\hline Sun Ultra 80 (450MHz/4MB L2) & 2 & 1.560 & 20352 & 384 & 1.8 \\
\hline Sun Ultra HPC $6000250 \mathrm{MHz}$ (1MB L2 Cache) & 4 & 1.560 & 15700 & 500 & 2.0 \\
\hline DEC 4100 5/300 (300 MHz) & 4 & 1.544 & 4436 & 500 & 2.4 \\
\hline Sun Fire $6800(900 \mathrm{MHz} / 8 \mathrm{MB}$ L2) & 1 & 1.509 & 28956 & 600 & 1.8 \\
\hline IBM SP2-T2 (66 MHz) & 8 & 1.5 & 9000 & 1680 & 2.1 \\
\hline Meiko CS2 & 16 & 1.5 & 10880 & 1952 & 2.9 \\
\hline NEC SX-3/11R (2.5 ns) & 1 & 1.5 & 2048 & 130 & 1.6 \\
\hline Parsytec GC/Power Plus (80 MHz) & 32 & 1.5 & 11000 & 3500 & 2.5 \\
\hline Convex SPP-1600(8 procs) $120 \mathrm{MHz}$ & 8 & 1.455 & 11000 & 750 & 1.9 \\
\hline Sun HPC $450(400 \mathrm{MHz})$ & 2 & 1.455 & 20252 & 960 & 1.6 \\
\hline SGI POWER CHALLENGE (75 MHz) & 6 & 1.430 & 2000 & 294 & 1.8 \\
\hline Intel iPSC/860 (40 MHz) & 72 & 1.4 & 9000 & 3500 & 2.9 \\
\hline Intel iPSC/860 (40 MHz) & 64 & 1.4 & 9000 & 3500 & 2.6 \\
\hline SGI Origin 2000 (195 MHz, 4MB cache) & 4 & 1.385 & 10000 & 1000 & 1.6 \\
\hline CRAY T3E-900 (450 MHz) & 2 & 1.323 & 7776 & 528 & 1.8 \\
\hline SGI POWER CHALLENGE (195 MHz, 2MB cache) & 4 & 1.305 & 10000 & 1000 & 1.6 \\
\hline Meiko Computing Surface (40 MHz) & 62 & 1.3 & 8500 & 3500 & 2.5 \\
\hline NEC SX-3/11 (2.9 ns) & 1 & 1.3 & 2816 & 192 & 1.4 \\
\hline SGI CHALLENGE (6.6ns) & 36 & 1.284 & 8000 & 2000 & 2.7 \\
\hline Sun Fire $6800(750 \mathrm{MHz} / 8 \mathrm{MB} \mathrm{L} 2 \$)$ & 1 & 1.260 & 15180 & & 1.5 \\
\hline SGI CHALLENGE (6.6ns) & 32 & 1.254 & 8000 & 2000 & 2.4 \\
\hline DEC AlphaServer 2100 5/250 & 4 & 1.2 & 4056 & 800 & 2.0 \\
\hline Fujitsu AP1000 & 256 & 1.2 & 18000 & 1600 & 1.4 \\
\hline IBM SP-1 & 16 & 1.2 & 12000 & 2300 & 2 \\
\hline Thinking Machines CM-200 (10 MHz) & 256 & 1.2 & 10752 & 4096 & 2.5 \\
\hline SGI POWER CHALLENGE (90 MHz) & 4 & 1.182 & 1000 & 240 & 1.4 \\
\hline SGI CHALLENGE (6.6ns) & 28 & 1.153 & 8000 & 2000 & 2.1 \\
\hline Alliant CAMPUS/800 (40 MHz) & 48 & 1.1 & 10024 & 3024 & 1.9 \\
\hline Sun Ultra HPC 450 (300 MHz) & 2 & 1.05 & 10944 & 192 & 1.2 \\
\hline SGI POWER CHALLENGE (75 MHz) & 4 & 1.046 & 14000 & 1000 & 1.2 \\
\hline Cray SV1-1-32 (300 MHz) & 1 & 1.044 & 7452 & 3501.2 & \\
\hline Convex SPP-1200(8 procs) $120 \mathrm{MHz}$ & 8 & 1.026 & 11000 & 750 & 1.9 \\
\hline SGI CHALLENGE/Onyx (6.6ns) & 24 & 1.014 & 8000 & 1000 & 1.8 \\
\hline Sun HPC 2 (300 MHz) & 2 & 1.01 & 7104 & 288 & 1.2 \\
\hline Convex SPP-1000(8 procs) $100 \mathrm{MHz}$ & 8 & 1.005 & 11000 & 550 & 1.6 \\
\hline SGI POWER CHALLENGE (75 MHz) & 4 & .993 & 1000 & 240 & 1.2 \\
\hline Intel iPSC/860 (40 MHz) & 48 & .98 & 7000 & 3000 & 1.9 \\
\hline Thinking Machines CM-5 & 16 & .98 & 6528 & 3008 & 2 \\
\hline nCUBE $2(20 \mathrm{MHz})$ & 512 & .958 & 15200 & 2240 & 1.2 \\
\hline HITACHI SR2201/4(150MHz) & 4 & .941 & 9720 & 1200 & 1.2 \\
\hline IBM PVS (40MHz) & 32 & .925 & 6000 & 1560 & 1.3 \\
\hline Intel Delta $(40 \mathrm{MHz})$ & 32 & .9 & 6000 & 2000 & 1.3 \\
\hline
\end{tabular}




\begin{tabular}{|c|c|c|c|c|c|}
\hline $\begin{array}{c}\text { Computer } \\
\text { (Full Precision) }\end{array}$ & $\begin{array}{l}\text { Number } \\
\text { of Procs } \\
\text { or Cores }\end{array}$ & $\begin{array}{c}\boldsymbol{R}_{\max } \\
\text { GFlop/s }\end{array}$ & $\begin{array}{c}N_{\max } \\
\text { Order }\end{array}$ & $\begin{array}{c}N_{1 / 2} \\
\text { Order }\end{array}$ & $\begin{array}{c}\boldsymbol{R}_{\text {Peak }} \\
\text { GFlop/s }\end{array}$ \\
\hline CRAY T3E (300 MHz) & 2 & 0.896 & 5280 & 384 & 1.2 \\
\hline SGI CHALLENGE/Onyx (6.6ns) & 20 & .866 & 7000 & 1000 & 1.5 \\
\hline Meiko Computing Surface (40 MHz) & 32 & .825 & 7000 & 3000 & 1.3 \\
\hline Meiko CS2 & 8 & .8 & 8064 & 1088 & 1.4 \\
\hline SGI CHALLENGE/Onyx (6.6ns) & 18 & .796 & 8000 & 1000 & 1.35 \\
\hline Sun Ultra 80 (450MHz/4MB L2) & 1 & .781 & 20352 & 192 & .9 \\
\hline NEC SX-3/1LR (2.5 ns) & 1 & .78 & 2304 & 112 & 0.8 \\
\hline Sun HPC $450(400 \mathrm{MHz})$ & 1 & 0.729 & 20252 & 960 & 0.8 \\
\hline SGI CHALLENGE/Onyx (6.6ns) & 16 & .702 & 8000 & 1000 & 1.2 \\
\hline SGI Origin 2000 (195 MHz, 4MB cache) & 2 & 699 & 10000 & 600 & .78 \\
\hline IBM RS/6000 Cluster (PARC) (62.5 MHz) & 8 & .694 & 10000 & 1500 & 1.0 \\
\hline Parsytec GC/Power Plus (80 MHz) & 16 & .68 & 7700 & 2200 & 1.3 \\
\hline NEC SX-3/1L (2.9 ns) & 1 & .67 & 2048 & 128 & .68 \\
\hline SGI POWER CHALLENGE (195 MHz, 2MB cache) & 2 & .663 & 10000 & 600 & .78 \\
\hline Intel iPSC/860 (40 MHz) & 32 & .64 & 6000 & 2500 & 1.3 \\
\hline SGI CHALLENGE/Onyx (6.6ns) & 14 & .631 & 8000 & 1000 & 1.05 \\
\hline SGI POWER CHALLENGE (90 MHz) & 2 & .601 & 1000 & 180 & .72 \\
\hline Fujitsu AP1000 & 128 & .566 & 12800 & 1100 & .71 \\
\hline SGI CHALLENGE/Onyx (6.6ns) & 12 & .554 & 7000 & 1000 & .9 \\
\hline IBM RS/6000 Cluster (PARC) (50 MHz) & 8 & .520 & 7500 & 1300 & .8 \\
\hline Sun Ultra HPC $450(300 \mathrm{MHz})$ & 1 & .52 & 10944 & 192 & .6 \\
\hline SGI POWER CHALLENGE (75 MHz) & 2 & .505 & 1000 & 180 & 6 \\
\hline Alliant CAMPUS/800 (40 MHz) & 24 & .504 & 7000 & 2492 & .96 \\
\hline Sun HPC $2(300 \mathrm{MHz})$ & 1 & .50 & 7104 & 288 & .6 \\
\hline Intel iPSC/860 (40 MHz) & 24 & .49 & 5000 & 2000 & .96 \\
\hline nCUBE $2(20 \mathrm{MHz})$ & 256 & .482 & 10784 & 1504 & .64 \\
\hline MasPar MP-1216 (80ns) & 16384 & .473 & 11264 & 1280 & .55 \\
\hline SGI CHALLENGE/Onyx (6.6ns) & 10 & .472 & 8000 & 1000 & .75 \\
\hline Intel Delta (40 MHz) & 16 & .45 & 4000 & 1000 & .64 \\
\hline Meiko Computing Surface (40 MHz) & 16 & .445 & 5000 & 2000 & .64 \\
\hline MasPar MP-1 (80 ns) & 16384 & .44 & 5504 & 1180 & .58 \\
\hline IBM RS/6000 Cluster (PARC) (50 MHz) & 6 & .404 & 7000 & 1200 & .6 \\
\hline ALR Revolution Quad 6 (4 Pentium $200 \mathrm{MHz}$ ) & 4 & .403 & 2750 & 530 & .8 \\
\hline MasPar MP-2204 (80ns) & 4096 & .374 & 5632 & 896 & .60 \\
\hline IBM RS/6000 Cluster (PARC) (62.5 MHz) & 4 & .37 & 5500 & 850 & .50 \\
\hline Intel iPSC/860 (40 MHz) & 16 & .36 & 4500 & 1500 & .64 \\
\hline SGI Origin 2000 (195 MHz, 4MB cache) & 1 & .356 & 10000 & 200 & .39 \\
\hline SGI POWER CHALLENGE (195 MHz, 2MB cache) & 1 & .334 & 10000 & 200 & .39 \\
\hline SGI POWER CHALLENGE (90 MHz) & 1 & .311 & 1000 & 100 & .36 \\
\hline IBM RS/6000 Cluster (PARC) (50 MHz) & 4 & .293 & 5500 & 1000 & .4 \\
\hline Fujitsu AP1000 & 64 & .291 & 10000 & 648 & .36 \\
\hline SGI POWER CHALLENGE (75 MHz) & 1 & .261 & 1000 & 100 & .3 \\
\hline nCUBE $2(20 \mathrm{MHz})$ & 128 & .242 & 7776 & 1050 & .32 \\
\hline HITACHI SR2201/1(150MHz) & 1 & .237 & 4860 & 420 & .3 \\
\hline Meiko Computing Surface (40 MHz) & 8 & .235 & 3500 & 750 & .32 \\
\hline
\end{tabular}




\begin{tabular}{|c|c|c|c|c|c|}
\hline $\begin{array}{c}\text { Computer } \\
\text { (Full Precision) }\end{array}$ & $\begin{array}{l}\text { Number } \\
\text { of Procs } \\
\text { or Cores }\end{array}$ & $\begin{array}{c}\boldsymbol{R}_{\max } \\
\text { GFlop/s }\end{array}$ & $\begin{array}{l}N_{\max } \\
\text { Order }\end{array}$ & $\begin{array}{c}N_{1 / 2} \\
\text { Order }\end{array}$ & $\begin{array}{c}\boldsymbol{R}_{\text {Peak }} \\
\text { GFlop/s }\end{array}$ \\
\hline Parsytec FT-400 (20 MHz) & 400 & .232 & 7999 & 814 & .6 \\
\hline Intel Delta (40 MHz) & 8 & .23 & 3000 & 1000 & .32 \\
\hline Intel iPSC/860 (40 MHz) & 8 & .19 & 3000 & 850 & .32 \\
\hline Meiko Computing Surface (40 MHz) & 4 & .121 & 2500 & 500 & .16 \\
\hline nCUBE $2(20 \mathrm{MHz})$ & 64 & .121 & 5472 & 701 & .15 \\
\hline Intel Delta (40 MHz) & 4 & .12 & 2000 & 500 & .16 \\
\hline MasPar MP-1204 (80ns) & 4096 & .116 & 5632 & 640 & .138 \\
\hline Intel iPSC/860 (40 MHz) & 4 & .10 & 2250 & 550 & .16 \\
\hline IBM RS/6000 (62.5 MHz) & 1 & .096 & 3000 & & .125 \\
\hline MasPar MP-2201 (80ns) & 1024 & .092 & 2816 & 448 & .15 \\
\hline Thinking Machines CM-5 & 1 & .068 & 1632 & 672 & .128 \\
\hline Meiko Computing Surface (40 MHz) & 2 & .062 & 1750 & 250 & .08 \\
\hline nCUBE $2(20 \mathrm{MHz})$ & 32 & .061 & 3888 & 486 & .075 \\
\hline Intel Delta (40 MHz) & 2 & .06 & 1500 & 500 & .08 \\
\hline Intel iPSC/860 (40 MHz) & 2 & .058 & 1500 & 400 & .08 \\
\hline nCUBE $2(20 \mathrm{MHz})$ & 16 & .032 & 5580 & 342 & .038 \\
\hline Meiko Computing Surface (40 MHz) & 1 & .031 & 1250 & & .04 \\
\hline MasPar MP-1201 (80ns) & 1024 & .029 & 2816 & 320 & .034 \\
\hline Intel iPSC/860 (40 MHz) & 1 & .024 & 750 & & .040 \\
\hline nCUBE $2(20 \mathrm{MHz})$ & 8 & .0161 & 3960 & 241 & .019 \\
\hline nCUBE $2(20 \mathrm{MHz})$ & 4 & .0080 & 2760 & 143 & .0094 \\
\hline nCUBE $2(20 \mathrm{MHz})$ & 2 & .0040 & 1280 & 94 & .0047 \\
\hline nCUBE $2(20 \mathrm{MHz})$ & 1 & .0020 & 1280 & 51 & .0024 \\
\hline
\end{tabular}

* The Numerical Wind Tunnel is not a commercial product; it is a computer of the National Aerospace Laboratory in Japan and is based on the Fujitsu vector processor board.

The CP-PACS (Computational Physics by Parallel Array Computer System) is not a commercial product, it is a computer of the University of Tsukuba, Japan. Hitachi modified several points in their SR-2201 computer. The processor, manufactured by Hitachi, is a custom superscalar processor. It is based on the PA-RISC Architecture enhanced with a PVP-SW (pseudo vector processor based on slide window registers) scheme.

** The IBM GF11 is an experimental research computer and not a commercial product.

*** Indicates Strassen Algorithm was used in computing the solution. Note the "achieved rate" is large than the "peak rate" for the computer. The rate of execution for this problem is based on the number of floating point operations divided by the time to solve the problem. The floating point operation count $2 / 3 n^{3}+O\left(n^{2}\right)$ is based on a conventional Gaussian Elimination implementation. Strassen's Algorithm reduced the number of operations actually performed. The results obtained for the computation presented here using Strassen Algorithm are as accurate as that from Gaussian Elimination. In general however Strassen's algorithm has less favorable stability properties than conventional matrix multiplication.

**** The Earth Simulator is not a commercial product; it is a computer of the Earth Simulator Center, the arm of the Japan Marine Science and Technology Center. It is based on vector processors that are manufactured by NEC.

The columns in Table 3 are defined as follows:

- $R_{\max }$ the performance in Gflop/s for the largest problem run on a machine.

- $N_{\max }$ the size of the largest problem run on a machine.

- $\quad N_{1 / 2}$ the size where half the $R_{\max }$ execution rate is achieved. 
- $R_{\text {peak }}$ the theoretical peak performance in Gflop/s for the machine.

In addition, the number of processors and the cycle time is listed. Full or half precision reflects the computation was computed using 64 or 32-bit floating point arithmetic respectively.

***** The algorithm used in obtaining this performance is based on an iterative refinement approach where both 32 and 64 bit floating point arithmetic is used. The method performs a LU factorization in 32 bit arithmetic and uses an iterative refinement approach which selectively uses 64 bit arithmetic to improve the solution to full 64 bit accuracy. The accuracy obtained is equivalent to the 64 bit implementation. In this case the $\mathrm{R}_{\text {peak }}$ is quoted for both the peak rates for 32 and 64 bit floating point arithmetic. A negative aspect of this approach is that the method need 1.5 times the memory of the approach used in the normal 64 bit implementation of LU factorization. See http://icl.cs.utk.edu/iter-ref/ for additional details.

\section{Acknowledgments}

I am indebted to the many people who have helped put together this collection.

\section{References}

1. J. Dongarra, J. Bunch, C. Moler, and G. W. Stewart. LINPACK User's Guide. SIAM, Philadelphia, PA, 1979.

2. J. J. Dongarra, I. S. Duff, D. C. Sorensen, and H. A. Van der Vorst. Solving Linear Systems on Vector and Shared Memory Computers. SIAM Publications, Philadelphia, PA, 1990.

3. C. Lawson, R. Hanson, D. Kincaid, and F. Krogh. Basic linear algebra subprograms for Fortran usage. ACM Trans. Math. Softw., 5:308-323, 1979. 